University of Louisville

ThinkIR: The University of Louisville's Institutional Repository

Electronic Theses and Dissertations

$12-2019$

\title{
The effects of nicotine and cigarette smoking on cardiac electrophysiology.
}

Affan B. Irfan

University of Louisville

Follow this and additional works at: https://ir.library.louisville.edu/etd

Part of the Cardiology Commons, Cardiovascular Diseases Commons, Medical Physiology Commons, and the Preventive Medicine Commons

\section{Recommended Citation}

Irfan, Affan B., "The effects of nicotine and cigarette smoking on cardiac electrophysiology." (2019).

Electronic Theses and Dissertations. Paper 3319.

https://doi.org/10.18297/etd/3319

This Doctoral Dissertation is brought to you for free and open access by ThinkIR: The University of Louisville's Institutional Repository. It has been accepted for inclusion in Electronic Theses and Dissertations by an authorized administrator of ThinkIR: The University of Louisville's Institutional Repository. This title appears here courtesy of the author, who has retained all other copyrights. For more information, please contact thinkir@louisville.edu. 


\title{
THE EFFECTS OF NICOTINE AND CIGARETTE SMOKING ON CARDIAC ELECTROPHYSIOLOGY
}

\author{
By \\ Affan B. Irfan \\ M,B.B,S Aga Khan University, 2008
}

\begin{abstract}
A Dissertation
Submitted to the Faculty of the School of Medicine of the University of Louisville

In Partial Fulfillment of the Requirements

for the Degree of
\end{abstract}

Doctor of Philosophy in Physiology and Biophysics

Department of Physiology

University of Louisville

Louisville, Kentucky

December 2019 



\title{
THE EFFECTS OF NICOTINE AND CIGARETTE SMOKING ON CARDIAC ELECTROPHYSIOLOGY
}

\author{
By \\ Affan B. Irfan \\ M,B.B,S Aga Khan University, 2008 \\ A Dissertation Approved on
}

November 27, 2019

by the following Dissertation Committee:

Dr Aruni Bhatnagar (Dissertation Director)

\begin{tabular}{c}
\hline Dr Alex Carll (Co-Director) \\
\hline Dr. Andrew P. DeFilippis \\
\hline Dr. Irving G. Joshua \\
\hline
\end{tabular}

Dr. Dale A. Schuschke 


\section{DEDICATION}

This dissertation is dedicated to my parents, Irfan Uddin and Farzana Irfan, (without whom

I would not have been here), my brother, Furqan Irfan, and sister Bismah Irfan, (without whom I would not have been the middle child), and my wife, Sommaya Naveed, and our daughter, Aiza Affan (without whom this dissertation would have been completed a year ago). 


\section{ACKNOWLEDGEMENTS}

Firstly, I am indebted to Dr. Aruni Bhatnagar, for giving me the opportunity to complete my Ph.D dissertation under his supervision, and also to Dr. Glenn Hirsch and the University of Louisville Cardiovascular Fellowship Program, for allowing me to participate in the Ph.D graduate studies during my fellowship training. I particularly would like to thank my mentors, Dr. Bhatnagar and Dr. Alex P. Carll, for their tireless guidance and "tough love" that pushed me out of my comfort zone (of just being a physician), and onto the path of a successful (and humble) scientist. I would like to thank the rest of my dissertation committee members (Dr. Andrew P. DeFilippis, Dr. Irving G. Joshua, and Dr. Dale A. Schuschke) for their support and invaluable advice. I am also especially grateful to Dr. Andrew P. DeFilippis for his expertise in clinical research studies that contributed greatly to this research. Moreover, I am thankful to Dr. Elsayed Soliman for his advice, collaboration, and contribution to projects related to this dissertation. This work would not have been possible without the administrative and financial support of Marshall University's Departments of Cardiology and Clinical Translational Services. I am especially grateful to research coordinators, Melissa Marcum and Scott Wiley, for their perseverance and unwavering commitment to this research study. I am greatly indebted to, and honored by, the encouragement and guidance from Dr. Christian Mueller, who introduced me to cardiovascular research and helped take the first steps of my scientific

pursuits. Lastly, I thank my dear family, especially my mother, father, brother, sister, and wife for their enduring love, support, and sacrifice. 


\section{ABSTRACT \\ THE EFFECTS OF NICOTINE AND CIGARETTE SMOKING ON CARDIAC ELECTROPHYSIOLOGY}

Affan B. Irfan

November 27, 2019

Cigarette smoking is a leading cause of preventable disease and premature death worldwide. The adverse effects of cigarette smoking, including proarrhythmia, are related to the mixture of chemicals, including nicotine (which sustains tobacco addiction). However, it remains unclear which individual tobacco smoke constituents and biological pathways mediate this increased risk. The purpose of this research was to explore the chronic effects of cigarette smoking, as well as compare the acute effects of nicotine and cigarette smoking, and the possible role of $\beta$-adrenoreceptors, on human cardiac electrophysiology. Chapter 1 is a comprehensive literature review of (a) the ex vivo and in vivo effects of nicotine and non-nicotine constituents of cigarette smoking on cardiac ion channels, (b) the direct and indirect effects of the autonomic nervous system on cardiac electrophysiology, and (c) studies of acute and chronic effects of cigarette smoking in humans. Chapter 2 consists of two studies in which we used cotinine levels to investigate the differences in baseline cardiac electrocardiogram between chronic smokers and nonsmokers, and to define smoking status and its burden. We also explored the relationship between urinary catecholamines, cotinine, and electrocardiographic changes. Chapter 3 features the $2 \times 2$ factorial experimental study designed to compare 
the acute effects of cigarette smoking and nicotine, with and without a $\beta$-blocker (propranolol). We found that chronic cigarette smoking was associated with a shortened PR segment at baseline, and that dopamine possibly mediates this effect. There was also (corrected) QT interval shortening with increased cotinine levels. This experimental study revealed that the non-nicotine constituents in cigarette smoking were mainly responsible for PR segment shortening, through $\beta$-adrenoreceptors. Other evidence revealed that, although nicotine in cigarette smoke is primarily responsible for sympathetic activation and (corrected) QT interval shortening, it is the non-nicotine constituents that depress the ST segment. Collectively, acute and chronic exposure studies indicate that smoking may promote cardiac arrhythmia, primarily via $\beta$-adrenoreceptors, causing acceleration of dromotropy and ischemia (non-nicotine mediated), and ventricular repolarization (nicotine-mediated). This research elucidated a major physiological mechanism driving the effect of cigarette smoking and nicotine on cardiac electrophysiology. Consequently, these findings will inform U.S. Food and Drug Administration of tobacco and nicotinecontaining products' impact on the human cardiac electrical system, and potentially help regulate alternative forms of nicotine delivery and protect public health. 


\section{TABLE OF CONTENTS}

\section{ACKNOWLEDGEMENTS}

PAGE iv

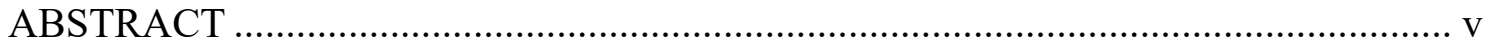

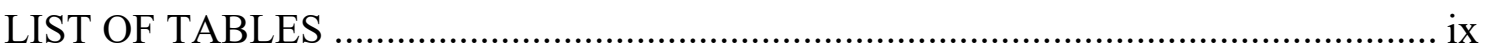

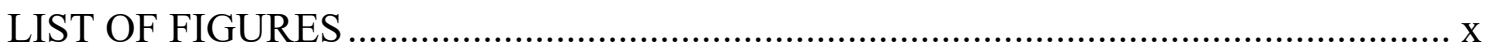

CHAPTER I. INTRODUCTION ......................................................................... 1

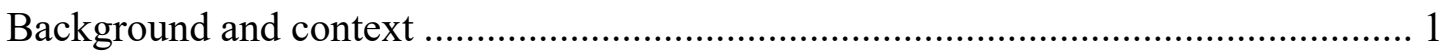

Clinical significance of cigarette smoking, nicotine, and cardiac

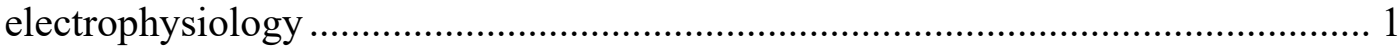

Effects of cardiac autonomic system and cigarette smoke on cardiac

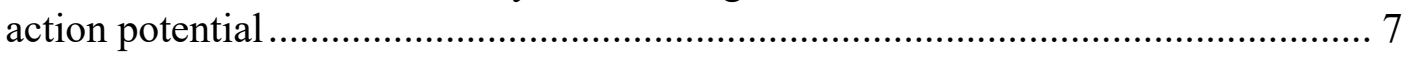

Literature review of effects of autonomic nervous system and smoking on

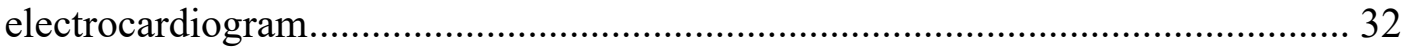

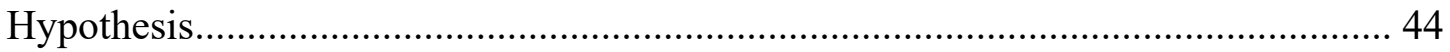

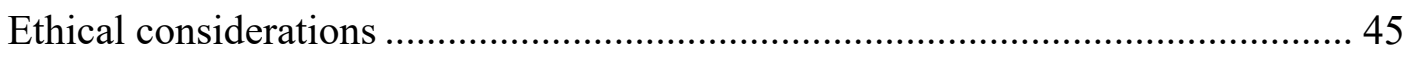

\section{CHAPTER II CHRONIC EFFECTS OF CIGARETTE SMOKING ON}

ELECTROCARDIOGRAM ........................................................................ 46

Cigarette smoking, ECG and interaction with Atrioventricular

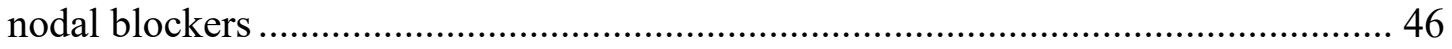

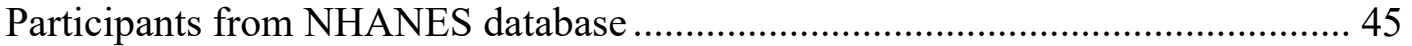

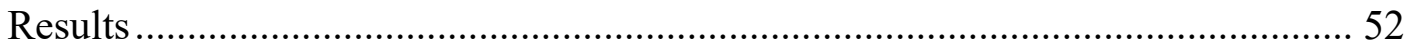

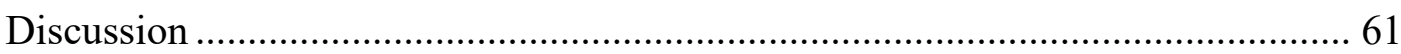

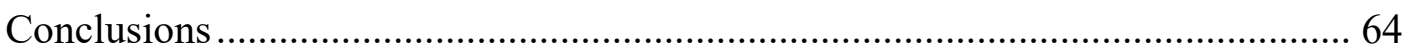

Effects of cigarette smoking on electrocardiogram mediated

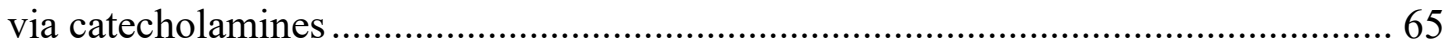

Participants from Louisville Healthy Heart Study ........................................... 65

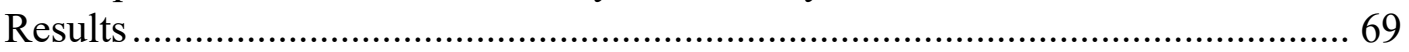

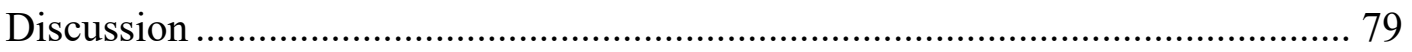

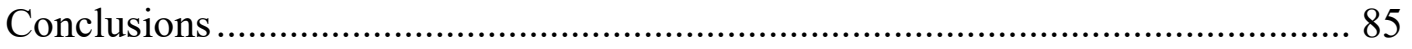




CHAPTER III ACUTE EFFECTS OF NICOTINE AND CIGARETTE




\section{LIST OF TABLES}

TABLE

PAGE

1.1 Summarizes the key studies on the acute effect of smoking on the circulating catecholamines. E - Epinephrine, NE - Norepinephrine. Dopa - Dopamine .......17

1.2 Potential mechanisms of indirect effects of cigarette smoking on human cardiac electrophysiology .30

1.3 Effects of cardiac receptors on chronotropy, dromotropy and inotropy.....

1.4 Major human studies on the chronic effects of smoking on atrial (A) and ventricular (B) electrocardiographic indices.

2.1.1 Baseline Participants Characteristics (Total $\mathrm{N}=5,653)$.................................52

2.1.2 Association between smoking status and abnormal PR interval and its components

2.1.3 Adjusted survey weighted linear regression between serum cotinine levels and PR interval, $\mathrm{P}$ duration and PR segment.....

2.1.4 Association between smoking status and abnormal corrected QT interval and its components

2.1.5 Adjusted survey weighted linear regression between serum cotinine levels and corrected QT interval, QRS duration and JT interval

2.2.1 Baseline characteristics

2.2.2 Comparison of creatinine-normalized urinary biomarkers (median [interquartile range]) among participants dichotomized into high and low atrial and atrioventricular conduction parameters

2.2.3 Estimated effects ( $\beta$-coefficients) of an increase in cotinine on PR interval, $\mathrm{P}$ wave, and $\mathrm{P}$ segment, with corresponding $\mathrm{P}$-values, from unadjusted and adjusted linear regressions.

3.1 Correlation between Area under the Curve of ECG parameters with change in Nicotine, Cotinine and 3-hyroycotinine (3-OH) among smokers on Cig-Day and NicS-Day and Non-smokers

3.2 Major findings of the experimental study 


\section{LIST OF FIGURES}

\section{FIGURE}

PAGE

1.1 Effect of Sympathetic nervous activation on human cardiac electrophysiology

1.2 Schematic diagram to illustrate the different direct sites of action for nicotine on the pathways of the cardiovascular autonomic nervous system

1.3 Direct effects of nicotine and non-nicotine constituents on cardiac ion channels and action potential. SNS - Sympathetic Nervous Activation

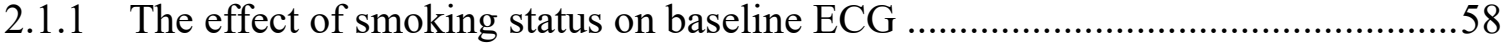

2.1.2 Panel A shows the distribution of PR segment (ms) and QTc interval (ms) with cotinine levels

2.2.1 Scatterplot and linear relationships of (A) PR interval, (B) P wave and (C) PR segment with log transformed urinary cotinine levels

2.2.2 Mediation analyses of cotinine, dopamine, and PR interval and PR segment.....

3.1 The Area Under the Curve for the raw changes $(\Delta)$ in ECG parameters from baseline for each visit day among smokers

3.2 ECG morphology in a typical participant during exposure on different days

3.3 The best fitting line to predict changes in Heart Rate, $\mathrm{P}$ amplitude, and QTc upon Cigarette and Nicotine exposures, and additional lines for PR segment and ST height upon Cigarette exposure.

3.4 Mediation analyses of changes in Nicotine, root mean squared of successive differences (RMSSD) and Heart Rate and ST-height among smokers on Cig-Day and NicS-Day and Non-smokers

4.1 Central illustration summarizing the impacts of nicotine and non-nicotine constituents of cigarette smoke on cardiac electrophysiology, and the pathways likely involved..... 


\section{CHAPTER I}

\section{INTRODUCTION}

\section{$\underline{\text { Background and context }}$}

Clinical significance of cigarette smoking, nicotine, and cardiac electrophysiology

\section{Cigarette smoke}

Cigarette smoking is the leading preventable cause of premature death. It is responsible for at least 480,000 premature deaths every year in the United States, with more than 41,000 of these deaths from exposure to secondhand smoke [1,2], out of which onethird are secondary to cardiovascular disease (CVD) [3]. The mortality of current smokers among both sexes is three times that of non-smokers $[4,5]$. Diseases attributable to smoking accounts for about $60 \%$ of smokers' deaths and the benefits of quitting smoking are dramatic across all age groups, with substantial gains in life expectancy, as compared to those who continue to smoke [5]. Furthermore, elevated CVD mortality has been seen even among patients smoking fewer than five cigarettes per day, suggesting a nonlinear dose-effect relationship [6]. Apart from the 21 common diseases formally attributed to cigarette smoking, there are diseases that have not been formally established as being caused by smoking (such as infections, hypertensive heart disease, renal failure, intestinal ischemia, and other respiratory diseases), and account for a significant excess in mortality [4]. The estimated annual smoking-related economic 
burden in the United States is between $\$ 289-332.5$ billion in direct medical care and other economic costs $[1,7]$. Smoking alsoappears to have a multiplicative interaction with other major CVD risk factors (elevated serum lipid levels, untreated hypertension, and diabetes) [2]. In 2016, 15.5\% (37.8 million) of U.S. adults were cigarette smokers. The prevalence of cigarette smoking was higher among adults who were male, 25-64 years old, of lower socio-economic status, of American Indian/Alaska Native descent or multiracial, and suffering from psychological distress [8].

\section{Nicotine and addiction}

Addiction to the nicotine in tobacco is the proximate cause of these diseases, because it sustains smoking behavior by acting on nicotinic cholinergic receptors in the brain to trigger the release of dopamine and other neurotransmitters [9]. Release of dopamine, glutamate, and gamma-Aminobutyric acid (GABA) [10] is particularly important in the development of nicotine dependence, and the extrahypothalamic corticotropin-releasing factor may play a key role in withdrawal [11, 12]. Nicotine addiction occurs when smokers rely on smoking to modulate mood and arousal, relieve withdrawal symptoms, or both. Therefore, the magnitude of public health harm caused by tobacco is inextricably linked to its addictive nature [9]. There is a continuum of risk for products that deliver nicotine, ranging from the most harmful combusted products (e.g., cigarettes) and electronic nicotine delivery systems (ENDS), to medicinal nicotine products.

Although significant research has been conducted to understand the pathophysiology of smoking-associated CVD, new research in this era has slowed in recent 
years. This lag in research is particularly harmful, as tobacco products have continued to evolve, and new products, such as ENDS, are a popular source of nicotine, especially among children and young adults [13]. In contrast with cigarettes, however, e-cigarettes generate an aerosol by heating a liquid, usually consisting of propylene glycol or vegetable glycerin, nicotine, and flavoring agents, without any combustion [14]. Despite almost complete lack of knowledge regarding the biological effects of these new tobacco products, ENDS have been implied to help conventional cigarette smokers quit or curtail smoking. ENDS manufacturers are making claims that these products pose fewer health risks and are much safer than smoking conventional cigarettes. This postulation is concerning because such health claims are likely to lead to increased ENDS use, and may even lead to an increase in nicotine dependence in the population at large. Although e-cigarettes deliver lower levels of carcinogens than do conventional cigarettes, they still expose users to high levels of ultrafine particles and other toxins, such as volatile organic compounds (VOCs) [15], that may substantially increase risks for cardiovascular and noncancerous lung disease, which account for more than half of all smoking-related deaths, at rates similar to conventional cigarettes [16].

The primary concern for nicotine in cigarette smoking is an addiction. Tobacco combustion products cause most of the adverse health effects from smoking, but some health concerns are related to nicotine. Many of these concerns are related to the ability of nicotine to release catecholamines, including hemodynamic effects (increase in heart rate, transient increases in blood pressure, vasoconstriction of coronary and other vascular beds), adverse effects on lipids, and induction of insulin resistance [17]. Both in vitro and in vivo 
animal studies suggest that nicotine may inhibit apoptosis, enhance angiogenesis, exacerbate atherosclerotic disease [18, 19], and produce endothelial dysfunction [20].

\section{Cardiac electrophysiology}

Basic cardiac electrophysiology is fundamental in understanding rhythmic cardiac function and electrical conduction, as well as changes in electrical activity associated with cardiac disease. The primary clinical tool for assessing cardiac electrical events is the electrocardiogram (ECG) [21]. The 12-lead ECG remains the most widely available, inexpensive, non-invasive, an indispensable tool for the diagnosis and prompt initiation of therapy in patients with acute coronary syndromes. It provides the most accurate means of diagnosing intraventricular conduction disturbances and arrhythmias, assessing cardiovascular risk and screening individuals in high-risk occupations and, in some cases, for participation in sports. As a research tool, it is used in long-term population-based surveillance studies and experimental trials of drugs with recognized or potential cardiac effects [22]. Therefore, ECG is used in clinical trials, as a valid, reliable, repeatable, quantitative method that is inexpensive and unbiased by clinical information.

Most ECGs used clinically are produced from digital signaling and interpreted by software using algorithms to assess cardiac rhythmicity, heart rate, heart rate variability (HRV), and intervals between conventional ECG landmarks, especially the PR, QRS, and QT intervals. These metrics provide essential insight into the autonomic function (HRV), atrioventricular conduction (PR interval and segment), ventricular depolarization (QRS), and repolarization (QT and JT). There are several major and minor ECG variables associated with cardiovascular mortality; namely $\mathrm{P}$ wave (duration, interatrial block, and 
deep terminal negativity of the P wave in V1), QT and Tpeak-Tend (Tp-Te) intervals, QRS duration and fragmentation, bundle branch block, ST segment depression and elevation, T waves (inverted, $\mathrm{T}$ wave axes), spatial angles between $\mathrm{QRS}$ and $\mathrm{T}$ vectors, premature ventricular contractions, and ECG hypertrophy criteria [23-25]. Apart from these traditional ECG markers, there is evidence for several other ECG intervals that have also been shown to be associated with total all-cause and cardiovascular-related mortality, such as PR interval [26-30], JT interval [31, 32] and Tp-Te [33-35].

Given its low cost, ubiquity, and safety, ECG is a useful candidate tool for screening and risk stratification of asymptomatic participants [36]. Deaths due to smoking-related CVD are generally preceded by a subclinical cardiovascular injury that may be detected early in the disease process [37]. To improve risk identification and stratification among asymptomatic smokers, and to aid in preventative measures, health professionals need more sensitive smoking-related markers of early cardiovascular damage. This research focus is particularly imperative in the new era of ENDs, as it remains unclear how ENDS might compare to cigarette smoke in severity and temporality of associated adverse outcomes.

\section{Implications for public health practice}

Some tobacco control researchers and advocates emphasize the need for strong policies that would protect future and current generations from new products that lead to nicotine addiction or serve as a gateway to cigarette smoking [16]. Others emphasize the different risks for disease associated with different tobacco and nicotine products, and argue that policies must prioritize reducing disease risk even if that means allowing for 
new products that may have high addiction potential [38]. As part of their framework announced in 2017, the Food and Drug Administration (FDA) — based on their recognition that nicotine makes tobacco products addictive, but that it does not directly cause smokers' cancer, lung disease, or heart disease — has proposed a regulation strategy designed to limit nicotine in cigarettes to a minimal or nonaddictive level [39]. The premise is that the mode of nicotine delivery, rather than the drug nicotine itself, is the key to reducing harm at a population level. This action has the promise of helping current users quit while preventing potential future smokers - youths, in particular-from becoming addicted via escalation from experimentation to regular smoking [40]. Modeling estimates that, by appropriate nicotine regulation, about 5 million adult smokers could quit within a year, and most youths and young adults - could avoid becoming regular smokers [41]. Whether this is the right approach is unclear, as through it, the FDA Center for Drug Evaluation Research has primarily been conducted on targeting smoking cessation rather than dependence on nicotine and alternate tobacco products [42]. As smokers find it difficult to achieve desired nicotine levels from low-nicotine cigarettes, they may seek to replace cigarettes with other tobacco products that deliver nicotine. The FDA expects that making cigarettes minimally addictive or nonaddictive would reduce tobacco-related harm by promoting smoking cessation or a complete migration to alternative, uncombusted products, and by reducing initiation. Although concrete evidence is lacking, there are concerns that ENDs use may renormalize smoking behavior, sustain dual-use, and initiate or maintain nicotine addiction [13]. ENDS use also could serve as a gateway to the initiation of smoking by ex-smokers. Unregulated e-cigarette use also has the potential to erode gains in smoking cessation and smoke-free laws [13]. Furthermore, the health effects of nicotine and e-cigarettes have not 
been well studied, and the potential harm incurred by long-term use of these devices remains completely unknown.

To complement Center for Tobacco Products (CTP) and CTP-funded scientific investigations to determine each product's risks, benefits, and net public health impact, the FDA encourages submission of information from additional rigorous research (e.g., outside research institutions, or a manufacturer in an application for FDA marketing authorization of a new product [40]). Hence, extensive new research is required to assess the health effects of nicotine in order to develop appropriate regulatory policies.

Effects of cardiac autonomic system and cigarette smoke on cardiac action potential

\section{Direct effects}

\section{Cardiac autonomic nervous system}

Sympathetic and parasympathetic control: Superimposed on the intrinsic cardiac control system are the major extrinsic factors-autonomic efferent postganglionic nerve terminals - which affect the secretion of hormones into circulation, and the release of chemicals directly onto the cardiomyocyte membrane [43]. The autonomic nervous system (ANS) plays a vital role in the genesis of several cardiac arrhythmias, both in the atria and in the ventricles. Modulation of the autonomic response is a complex process, in which the final effect is the product of interactions among central, peripheral, and intracardiac components. Autonomic activation alters not only the heart rate, conduction, and hemodynamics, but also the cellular and subcellular properties of individual myocytes.

The cardiac ANS is divided into extrinsic (fibers that mediate connections between the heart and the nervous system) and intrinsic (fibers facilitating function within the 
pericardial sac) components. The extrinsic cardiac ANS is divided into sympathetic and parasympathetic components. The fibers of the sympathetic nervous system (SNS) are largely derived from major autonomic ganglia along the cervical and thoracic spinal cord. The parasympathetic nervous system (PNS) originates predominantly in the nucleus ambiguus of the medulla oblongata and is carried almost entirely within the vagus nerve. In addition to the extrinsic cardiac ANS, the heart is also innervated by an exquisitely complex intrinsic cardiac ANS, with the vast majority of these ganglia organized into ganglionated plexi (GP) on the surface of the atria and ventricles, particularly at the sinus node, atrioventricular node and pulmonary vein-left atrium junction [43, 44]. There is a group of complex extracardiac and intrinsic cardiac neurons that comprise a local distributive network, process (both centripetal and centrifugal) information in cardiac control, and imply the presence of local information processing [45]. Furthermore, the influences of sympathetic and parasympathetic stimulation exert not only different effects on atrial and ventricular myocytes, but also during normal and diseased states [44]. Generally speaking, increased cardiac sympathetic efferent neuronal tone increases cardiac chronotropy, dromotropy, and inotropy; the reverse holds for the effects exerted by medullary (parasympathetic) efferent preganglionic neurons [43].

Sympathetic nerve stimulation results in well-defined changes in electrophysiological properties at the cellular and tissue levels, including enhanced conduction in working myocardium and shortening of the action potential duration and refractory periods. Sympathetic control of cardiac electrical activity is mediated by the activation of $\beta$-adrenergic receptors that regulate the activity of select ion channel proteins via cyclic adenosine monophosphate (cAMP)-dependent protein kinase A (PKA), or by 
direct binding of cAMP to channel subunits. The activation of $\beta$-receptors regulates the function of many ion channels in the heart, including $\mathrm{Na}^{+}, \mathrm{K}^{+}$, and $\mathrm{Ca}^{2+}$ channels [46].

Sodium and Calcium channels: $\beta$-adrenergic receptors' regulation of cardiac $\mathrm{Na}^{+}$ channels may occur via several distinct mechanisms. PKA-dependent and -independent (a more recent and poorer defined pathway) signaling pathways impact cardiac $\mathrm{Na}^{+}$channel function. The indirect pathway engages canonical signaling, including PKA phosphorylation of the $\mathrm{Na}^{+}$channel $\alpha$-subunit. PKA-independent regulation (direct regulation) involves ion channels, such as $\mathrm{Nav}_{\mathrm{v}} 1.5, \mathrm{Cav}_{\mathrm{v}} 1.2$, and $\mathrm{K}_{\mathrm{V}} 1.5$, which are enriched in caveolae and colocalized with Cav3. Caveolae are ready reservoirs of select cell membrane proteins; $\beta$-adrenergic receptor stimulation opens caveolae through a Gasinvolved, PKA-independent pathway, and increases membrane density of resident ion channels [47]. In addition to caveolin-associated augmentation of surface expression and channel phosphorylation, sympathetic activation increases L-type $\mathrm{Ca}^{2+}$ channel activity and intracellular $\mathrm{Ca}^{2+}$. Phosphorylation of the Nav1.5 channel results in the alteration of the voltage-dependent kinetics and whole-cell INa amplitude [48]. There is limited evidence for direct regulation of cardiac $\mathrm{Na}^{+}$currents by parasympathetic activity, but the reversal of the effects of $\beta$-adrenergic receptor stimulation by acetylcholine has been described [49], likely via pre-synaptic inhibition upon muscarinic receptor activation [50]. $\beta$-adrenergic receptor stimulation appears to alter the voltage-gated calcium current via a dual mechanism, perhaps similar to that demonstrated previously for sodium channels [51]. The L-type cardiac calcium channel, CaV1.2a, is phosphorylated by PKA, which increases open channel probability, and subsequently the overall cellular calcium current [47]. 
Potassium channels: Sympathetic activation can directly affect all potassium currents, including Ito (responsible for transient outward current and level of the plateau in action potential). The delayed rectifier $\mathrm{K}$ currents IKur, IKr, and IKs are slowly activating outward currents that play major roles in the control of repolarization. $\beta$-adrenergic stimulation regulates IKr through the activation of PKA (an inhibitory effect) and elevation of c-AMP (a stimulatory effect through binding to the cyclic nucleotide binding domain of the channel), whereas $\alpha$-adrenergic stimulation is inhibitory. $\beta$-adrenergic stimulation also accelerates repolarization by augmenting IKs via PKA-dependent phosphorylation of Kv7.1 (also termed KvLQT1, encoded by the Kcnq1 gene) [52-54], and $\beta$-blockers prolong transmural dispersion of repolarization and action potential duration [55]. However, there is a potential inhomogeneity of effects of $\beta$-adrenergic stimulation on potassium channels across different species, which also vary by stimulus and disease states [50]. Vagal stimulation produces the opposite effects. Vagal stimulation releases acetylcholine, which then activates a potassium current and an inward-rectifying $\mathrm{K}^{+}$current (IKAch), following stimulation of muscarinic (M2) receptors that hyperpolarizes the membrane potential and abbreviates the action potential, slowing the Phase 4 depolarization of pacemaker cells [52, 56]. In contrast, Liang et al. tested acetylcholine shortened action potential duration in $e x$ vivo rat ventricular tissue, and the effect was inhibited by a G-protein-coupled inward rectifier potassium (GIRK) channel blocker [57]. Furthermore, the muscarinic stimulation can also partially reduce the amplitude of the L-type $\mathrm{Ca}^{2+}$ current by inhibiting adenylate cyclase. Therefore, vagal stimulation's effect on cardiac repolarization is complex and may differ by species, phenotype, or stimulus. 
As illustrated in Figure 1.1, sympathetic dominance in humans produces an increase in upstroke velocity, amplitude, and conduction velocity, as well as a decrease in the effective refractory period. These effects are mainly due to stimulation of $\beta$-adrenergic receptors and the resulting augmentation of INa, ICa, IKr, and IKs. Therefore, acute sympathetic neural activation results in the shortening of RR interval, $\mathrm{P}$ wave, PR interval, PR segment, QRS duration, QT, and Tp-Te. 


\section{Sympathetic nervous activation}

A

Fast-response action potentials
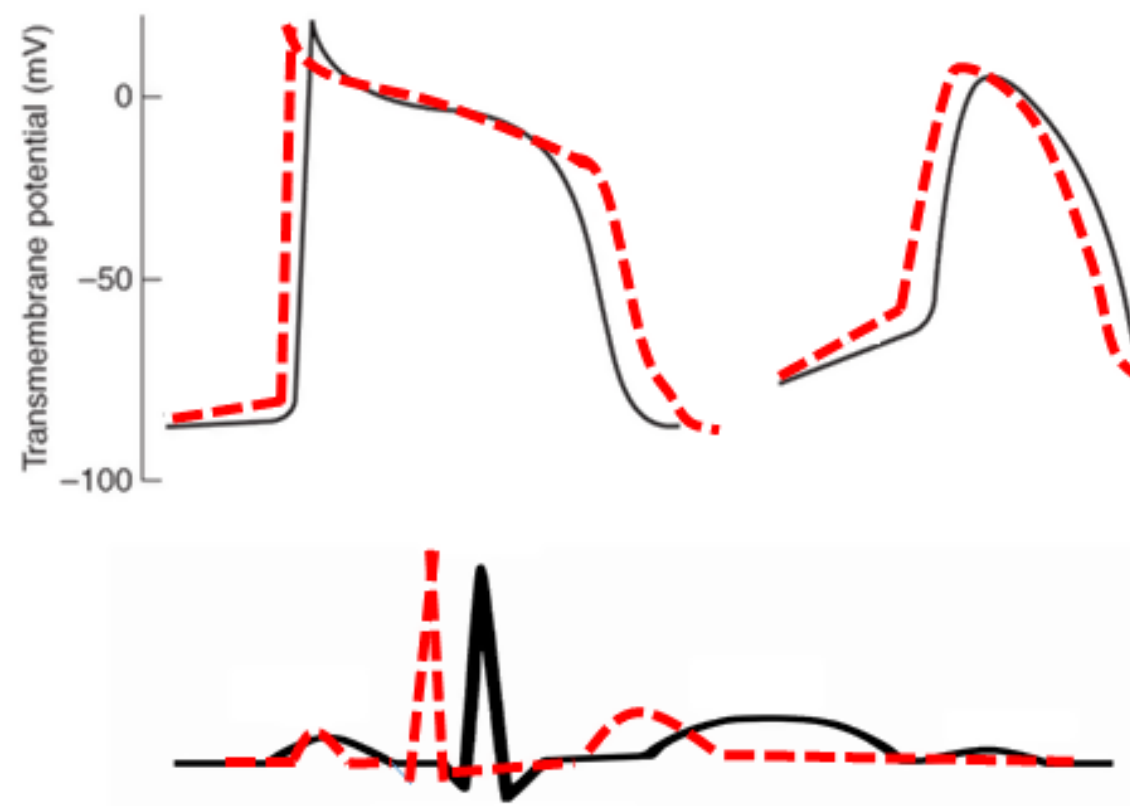

Figure 1.1 Effect of Sympathetic nervous activation on human cardiac electrophysiology

\begin{tabular}{lll}
\hline \multicolumn{1}{c}{ References } & Effects of SNS activation & \multicolumn{1}{c}{ ECG intervals } \\
\hline$[58]$ & Short & P wave / P-P wave \\
{$[59,60]$} & Short & PR interval \\
{$[61]$} & Short & PR segment \\
{$[62,63]$} & Short & QRS duration \\
{$[64-66]$} & Short & QT interval \\
\hline
\end{tabular}




\section{Nicotine}

Cardiac autonomic nervous system: Nicotinic acetylcholinergic receptors (nAchRs) mediate the neural transmission at the ganglia of both branches of the ANS. However, different nicotinic receptors at the ganglia play distinct roles in sympathetic and parasympathetic cardiovascular responses (Figure 1.2). Specifically, activation of $\alpha 4 \beta 2$ nAchRs elicits a parasympathetic cardiovascular response, and activation of $\alpha 7 \mathrm{nAchRs}$ elicits a sympathetic cardiovascular response $[67,68]$. Neff et al. used the perforated patchclamp technique in a visualized rat brain stem slice to identify three potential sites of action by which nicotine increases the activity of cardiac vagal neurons: (a) direct activation of postsynaptic ligand-gated nicotinic channels in cardiac vagal neurons, (b) different presynaptic terminals, and (c) postsynaptic glutamatergic terminals [69]. Moreover, in various animal models and species, nicotine has been shown to evoke norepinephrine release from both the peripheral postganglionic sympathetic nerve endings and adrenal medulla $[70,71]$. Another potentially sympathoexcitatory mechanism of nicotine involves the inhibition of neuronal nitric oxide synthase, which decreases central nitric oxide availability, thereby removing its tonic inhibitory effect on central sympathetic outflow $[72]$. 


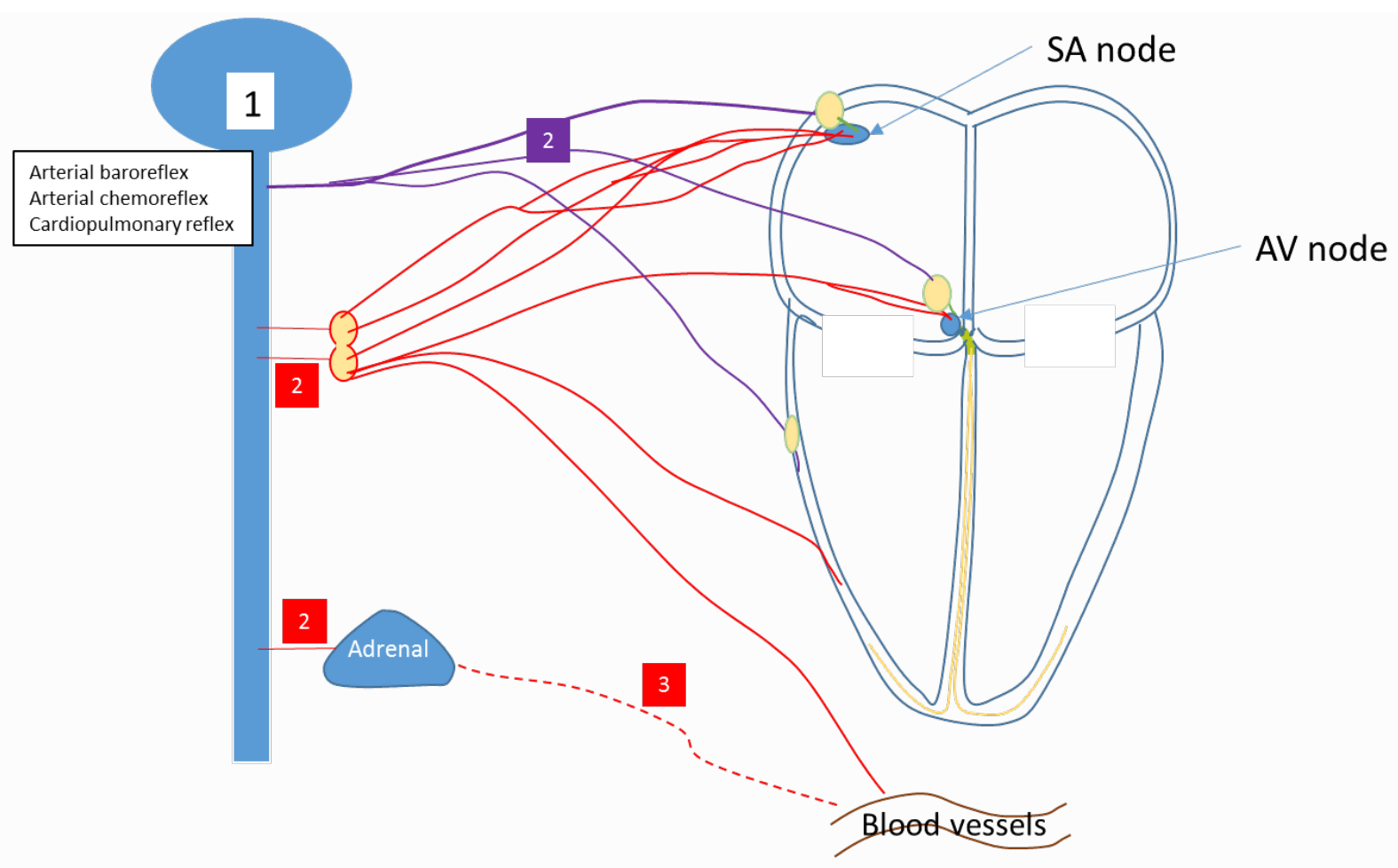

Schematic diagram to illustrate the different direct sites of action for nicotine on the pathways of the cardiovascular autonomic nervous system. Nicotine acts on the Nicotinic acetylcholine receptors (nACHRs) in 1) Central nervous system 2) Pre-ganglion (at both sympathetic and parasympathetic neurotransmission) and at 3) Post ganglion and chromaffin cells in adrenal glands at sympathetic nerve transmission.

Figure 1.2 Schematic diagram to illustrate the different direct sites of action for nicotine on the pathways of the cardiovascular autonomic nervous system

SA - Sinoatrial node. AV - Atrioventricular node. 
Cardiac sodium and calcium channels: The binding of nicotine to the extracellular binding site of the nicotinic acetylcholine receptor leads to a conformational change of the central pore, which results in the influx of sodium and calcium ions $[73,74]$. In addition to the increase in intracellular calcium concentration facilitated by sodium influx through the nicotine receptor [73], nicotine also evokes calcium influx by direct activation of voltagedependent calcium channels [75-77]. More specifically, the L-type Ca2+ channels-Cav 1.2 channel, in particular - have been implicated in nicotine addiction, and are controlled by the SNS and stimulated by nicotine $[78,79]$. Therefore, several studies have consistently shown that nicotine can both indirectly (catecholamine-mediated) and directly activate voltage-gated sodium and calcium ion channels, depolarize membrane potential, and increase cardiac contractility [80-83].

Cardiac potassium channels: On the other hand, nicotine directly blocks multiple types of potassium currents, (A-type $\mathrm{K}+$ currents (Ito current/Kv4.3 channel), delayed rectifier $\mathrm{K}+$ currents $(\mathrm{IKr} / \mathrm{HERG})$ and inward rectifier $\mathrm{K}+$ currents (Ik1/Kir2.1), independent of nicotinic receptor stimulation or catecholamine release [84-86]. However, the ex vivo studies on the effects of nicotine on the duration of action potential have been inconsistent. Some found that nicotine shortens the action potential duration (particularly phase 2) [83], whereas others found that nicotine prolongs it [87]. These discrepancies could be related to the time from drug administration [82], drug concentration [80,88] or the concomitant increase in force [89]. The shorter phase 2 could also reflect nicotine stimulation of L-type $\mathrm{Ca} 2+$ channel, which, together with potassium channels, dictate the duration of this phase of the action potential. Similar dose-dependent effects of nicotine 
are seen in the sinoatrial node, where low doses of nicotine reduce $[81,88]$-and large doses increase [88] — the spontaneous cycle length of sinoatrial node pacemaker cells.

At physiological doses, nicotine can indirectly (through the sympathetic nervous system) and directly (via immediate effects on cardiac ion channels) stimulate sodium and calcium channels and block potassium channels. Per the pathways mentioned above, nicotine is expected to induce multiple alterations in the surface ECG, including shortening of the P wave, PR interval, PR segment, and QRS duration, and prolongation of QT corrected $(\mathrm{QTc})$ and $\mathrm{Tp}-\mathrm{Te}$. However, very few researchers have attempted to explore the effects of nicotine on all ECG intervals in humans.

\section{Cigarette smoking and its non-nicotine constituents}

Cardiac autonomic nervous system: Cigarette smoke-induced cardiovascular effects are at least partly due to stimulation of sympathetic neurotransmission, and can theoretically manifest at four different sites of the sympathetic nervous system: the brain, pre-ganglionic and post-ganglionic sympathetic nerves, and the adrenal medulla [71]. Acute cigarette smoking increases efferent sympathetic nerve activity, primarily via the release of the catecholamines norepinephrine, and epinephrine (Table 1.1). This catecholamine release increases myocardial work and oxygen consumption through an increase in blood pressure, heart rate, and myocardial contractility [19]. However, several studies suggest that the primary effect is from direct pharmacologic stimulation of nicotinic acetylcholine receptors, as well as the catecholamine release from localized peripheral postganglionic sympathetic nerve endings and the adrenal medulla $[90,91]$. Despite evidence that sympathetic tone is increased during smoking, either by increased release or 
decreased clearance of catecholamines at neuroeffector junctions via inhibition of monoamine oxidase (MOA) [92], Grassi et al. revealed that the central sympathetic activity is inhibited, presumably via counteracting baroreceptor reflexes [90]. 
Table 1.1 Summarizes the key studies on the acute effect of smoking on the circulating catecholamines. E - Epinephrine, NE -

\section{Norepinephrine. Dopa - Dopamine}

\begin{tabular}{|c|c|c|c|c|c|c|c|}
\hline $\begin{array}{l}\text { Author } \\
\text { Date } \\
\text { Journal }\end{array}$ & Participants & Cigarette & $\begin{array}{l}\text { Plasma/ } \\
\text { urine }\end{array}$ & Sampling frequency & Catecholamines & Result & $\begin{array}{l}\text { Comment } \\
\text { /Nicotine }\end{array}$ \\
\hline $\begin{array}{l}\text { Cryer } \\
1976 \\
\text { NEJM }\end{array}$ & $\begin{array}{l}10 \text { healthy men } \\
(24-42 \mathrm{y})\end{array}$ & $\begin{array}{l}\text { Two standard non- } \\
\text { filtered cigarettes in } \\
10 \text { minutes }\end{array}$ & Plasma & $\begin{array}{l}-10,0,2.5,5.0,7.5 \\
10,12.5,15.0,17.5 \\
20,25,30 \text { (mins) }\end{array}$ & $\mathrm{E}, \mathrm{NE}$ & $\begin{array}{l}\text { Max at } 10 \text { and } \\
12.5 \text { min for } \mathrm{E} \\
\text { and } \mathrm{NE}\end{array}$ & $\begin{array}{l}\text { E significantly } \\
\text { higher even at } \\
\text { end. }\end{array}$ \\
\hline $\begin{array}{l}\text { Siess } \\
\text { Circulation } \\
1982\end{array}$ & $\begin{array}{l}6 \text { healthy men, } \\
\text { mean age } 30 y\end{array}$ & $\begin{array}{l}\text { Two cigarettes of } \\
1.6 \mathrm{mg} \text { nicotine each }\end{array}$ & Plasma & End of smoking & $\mathrm{E}, \mathrm{NE}$ & $\begin{array}{l}\text { Increased from } \\
\text { baseline }\end{array}$ & \\
\hline $\begin{array}{l}\text { Mundal } \\
1988 \\
\text { Am J } \\
\text { Hypertens }\end{array}$ & $\begin{array}{l}18 \text { normotensive, } \\
13 \text { hypertensive } \\
\text { white men (mean } \\
\text { age } 42 \mathrm{y} \text { ) }\end{array}$ & $\begin{array}{l}\text { Two cigarettes, } 1.7 \\
\text { mg nicotine }\end{array}$ & Plasma & $\begin{array}{l}\text { Baseline, after each } \\
\text { cigarette and after } \\
60 \text { minutes from } \\
\text { start of smoking }\end{array}$ & $\mathrm{E}, \mathrm{NE}$ & $\begin{array}{l}\text { E increased after } \\
\text { second cigarette } \\
\text { (not after } 60 \mathrm{~min} \text { ) } \\
\text { only among } \\
\text { hypertensive } \\
\text { group }\end{array}$ & $\begin{array}{l}15 \mathrm{ng} / \mathrm{ml} \text { after } \\
1^{\text {st }} \text { cigarette } \\
\text { and } 23 \mathrm{ng} / \mathrm{ml} \\
\text { after second } \\
\text { cigarette }\end{array}$ \\
\hline $\begin{array}{l}\text { Niedermaier } \\
1993 \\
\text { Circulation }\end{array}$ & $\begin{array}{l}8 \text { healthy, } 5 \text { men } \\
(19-44 \text { y) }\end{array}$ & $\begin{array}{l}\text { Low and medium- } \\
\text { nicotine research } \\
\text { cigarette (30-45 } \\
\text { mins apart) }\end{array}$ & Plasma & $\begin{array}{l}10 \text { mins after each } \\
\text { smoking period (to } \\
\text { coincide with max } \\
\text { catecholamines) }\end{array}$ & $\mathrm{E}, \mathrm{NE}$ & No difference & $\begin{array}{l}7 \text { and } 16 \\
\mathrm{ng} / \mathrm{ml} \text { after } \\
\text { low and } \\
\text { medium } \\
\text { cigarette }\end{array}$ \\
\hline $\begin{array}{l}\text { Grassi } \\
1994 \\
\text { Circulation }\end{array}$ & $\begin{array}{l}8 \text { men, } 1 \text { woman, } \\
(21-48 \text { y) }\end{array}$ & $\begin{array}{l}\text { One filtered } \\
\text { cigarette } 1.1 \mathrm{mg} \\
\text { nicotine (within } 5 \\
\text { mins) }\end{array}$ & Plasma & End of smoking & $\mathrm{E}, \mathrm{NE}$ & $\begin{array}{l}\text { Significant } \\
\text { increase }\end{array}$ & $\begin{array}{l}\text { After } 1^{\text {st }} \\
\text { cigarette } 44 \\
\mathrm{ng} / \mathrm{ml}\end{array}$ \\
\hline $\begin{array}{l}\text { Krzysztof } \\
1998 \\
\text { Circulation }\end{array}$ & $\begin{array}{l}14 \text { healthy } \\
\text { smokers ( } 13 \\
\text { men). Mean age } \\
23 \text { y }\end{array}$ & $\begin{array}{l}\text { Two cigarettes } \\
\text { containing } 1.1 \mathrm{mg} \\
\text { nicotine }(5 \mathrm{mins} \\
\text { apart }) .45 \text { mins later } \\
\text { smoked third } \\
\text { cigarette }\end{array}$ & Plasma & $\begin{array}{l}\text { Outset of study and } \\
3 \text { mins after second } \\
\text { and third cigarette }\end{array}$ & $\mathrm{NE}$ & $\begin{array}{l}\text { Increased from } \\
171 \text { (baseline) to } \\
189 \text { (after } 2 \\
\text { cigarettes) and } \\
214^{*}\left(3^{\text {rd }}\right. \\
\text { cigarette) }\end{array}$ & $\begin{array}{l}17 \mathrm{ng} / \mathrm{ml} \text { and } \\
20 \mathrm{ng} / \mathrm{ml} \text { of } \\
\text { nicotine, after } \\
2^{\text {nd }} \text { and } 3^{\text {rd }} \mathrm{cig} . \\
\text { Smoked third }\end{array}$ \\
\hline
\end{tabular}




\begin{tabular}{|c|c|c|c|c|c|c|}
\hline $\begin{array}{l}\text { Gourlay } \\
1997 \\
\text { Clin } \\
\text { Pharmacol } \\
\text { Ther }\end{array}$ & $\begin{array}{l}12 \text { healthy male } \\
\text { smokers, mean } \\
\text { age } 38 \mathrm{y}\end{array}$ & $\begin{array}{l}6 \text { got nicotine nasal } \\
\text { spray }(1 \mathrm{x} 0.5 \mathrm{mg} \text { in } \\
\text { each nostril) and } \\
\text { other } 6 \text { did smoking } \\
\text { (usual brand) }\end{array}$ & Plasma & $\begin{array}{l}0,2,4,6,8,10,15 \\
20,25,30,45 \text {, and } \\
60 \text { mins }\end{array}$ & $\mathrm{E}, \mathrm{NE}$ & $\begin{array}{l}\text { Venous } \mathrm{E} \text { and } \mathrm{NE} \\
\text { remain } \\
\text { unchanged }\end{array}$ \\
\hline $\begin{array}{l}\text { Bragg } \\
1956 \\
\text { J Appl Physi }\end{array}$ & $\begin{array}{l}11 \text { healthy young } \\
\text { men }\end{array}$ & $\begin{array}{l}\text { Their choice of } \\
\text { standard cigarettes } \\
\text { during } 2 \text { / } 8 \text { hour ( } 4 \\
\text { and } 10 \text { cigs) } \\
\text { cigarette smoking }\end{array}$ & Urine & $\begin{array}{l}\text { 8-hour urine } \\
\text { collection }\end{array}$ & $\mathrm{E}, \mathrm{NE}$ & $\begin{array}{l}\text { E increased, NE } \\
\text { unchanged }\end{array}$ \\
\hline $\begin{array}{l}\text { Benowitz } \\
1993 \\
\text { JACC }\end{array}$ & $\begin{array}{l}12 \text { healthy male } \\
\text { smokers, } 31-65 \\
\text { y }\end{array}$ & $\begin{array}{l}\text { (own choice), } \\
\text { transdermal nicotine } \\
(21 \mathrm{mg} / \mathrm{d}) \text { and } \\
\text { placebo }\end{array}$ & Urine & $\begin{array}{l}24 \mathrm{hr} \text { Urine } \\
\text { collected day } 5\end{array}$ & Dopa, E, NE & $\begin{array}{l}\text { Significantly } \\
\text { higher with } \\
\text { smoking }\end{array}$ \\
\hline $\begin{array}{l}\text { Benowitz } \\
1989 \\
\text { Ann Intern } \\
\text { Med }\end{array}$ & $\begin{array}{l}8 \text { healthy male } \\
\text { smokers }\end{array}$ & $\begin{array}{l}\text { oral snuff, chewing } \\
\text { tobacco, and } \\
\text { cigarettes }\end{array}$ & Urine & $\begin{array}{l}\text { 24-hour urine after } \\
\text { 3- or 4-day blocks }\end{array}$ & Dopa, E, NE & $\begin{array}{l}\text { Significantly } \\
\text { higher with } \\
\text { smoking }\end{array}$ \\
\hline
\end{tabular}




\section{Non-nicotine constituents:}

In addition to nicotine, several other cigarette components have been implicated in inducing proarrhythmia, such as VOCs (aldehydes [acrolein, formaldehyde, acetaldehyde, crotonaldehyde], benzene, toluene), particulate matter, gases (carbon monoxide), and polycyclic aromatic hydrocarbons. It is estimated that about $90 \%$ of non-cancer mortality from tobacco smoke is due to aldehydes (acrolein, formaldehyde, and acetaldehyde) [93]. In animal models, a single exposure to acrolein significantly increased HRV and arrhythmia independent of heart rate, possibly through activation of the transient-receptor potential ankyrin-1 channel (TRPA1), an irritant receptor channel found in the airways [94, 95]. Treatment with atenolol reduced this response, whereas atropine enhanced it, suggesting parasympathetic dominance and sympathetic modulation [95]. Non-nicotinic alkaloids, such as nornicotine or anabasine (which represent $8-12 \%$ of total alkaloid content in tobacco), also exert an agonistic activity on nicotinic receptors [96]. Acute exposure to toluene, the most abundant aromatic compound in mainstream smoke from full-flavored cigarettes, enhances heart rate and blood pressure at baseline conditions, primarily due to systemic increases in circulating catecholamines [97, 98], and the enhanced protein expression of $\beta 1$ adrenergic receptors [97]. Additionally, intravenous acetylaldehyde in anesthetized cats has been found to increase systemic blood pressure and heart rate (presumably from release of endogenous catecholamines from cardiac tissue) [99]. Formaldehyde has been shown to induce significant bradycardia and negative inotropic responses in both in situ preparations of guinea pig and rabbit hearts, and in vitro cardiac preparations [100]. Notably, the negative chronotropic effect of formaldehyde in 
animals seems to be caused mainly by the inhibition of sympathetic nervous activity through the central nervous system [100].

Several non-nicotine cigarette ingredients have long been recognized as cardiotoxic and linked with cardiac electrical activity disturbance and arrhythmias, such as aromatic compounds [101], carbon monoxide [102], and aldehydes [99]. However, literature on their individual drug concentration effects on cardiac ion channels and the action potential is scarce.

Cardiac sodium and calcium channels: Toluene inhibits activated currents through ligand and voltage-gated sodium and calcium channels [97, 103-106]. Phenol has been shown to exert a dose-dependent negative inotropic effect in an isolated mammalian cardiac muscle, possibly via blocking calcium channels [107]. In a patch-clamp electrophysiology and confocal imaging experiment with isolated ventricular myocytes, carbon monoxide activated nitric oxide synthase. This led to the nitric-oxide-mediated nitrosylation of Nav1.5, as well as increased the sustained (late) component of the inward $\mathrm{Na}(+)$ current, and inhibited peak Nav1.5 current amplitude, ultimately resulting in prolonged action potential and associated intracellular $\mathrm{Ca}(2+)$ transient [102]. In addition, carbon monoxide inhibits native rat cardiomyocyte L-type $\mathrm{Ca} 2+$ currents and the recombinant $\alpha 1 \mathrm{C}$ subunit of the human cardiac L-type Ca2+ channel [108, 109]. Formaldehyde and other aldehydes have previously been shown to cause dramatic deceleration of sodium inactivation, depress Ina, and prolong action potential [110-113]. On the other hand, acetaldehyde has also been shown to increase the ICa, and thereby increase the contractile force [114], Its effects can be potentiated by the additional stimulation of $\alpha$-adrenergic receptors [115]. Several other cigarette constituents have also 
been found to, at minute quantities, significantly inhibit sodium and calcium channels in isolated cardiac cells, such as cyanide [116, 117], lead [118], and cadmium [119]. Particulate matter, encountered during cigarette smoking, is also a significant cause of cardiovascular morbidity and mortality. Particulate matter has been shown to dysregulate prominent $\mathrm{Na}^{+}$and $\mathrm{K}^{+}$channel pathway genes [120], and carotid body sensitivity [120].

Cardiac potassium channels: Aldehydes have been proven to considerably inhibit IK1 and Ito in animal atrial and ventricular myocytes [121-123]. In one study, ethanol and acetaldehyde inhibited the $(\mathrm{Na}++\mathrm{K}+)$-activated ATPase activity of plasma membranes prepared from a guinea-pig heart in a dose-dependent manner [124]. In addition, several volatile agents found in cigarette smoke, commonly used as anesthetics, have been shown to inhibit G-protein-coupled inwardly rectifying potassium channels [125]. Benzene derivatives inhibit delayed rectifier $\mathrm{K}+$ currents [126], specifically IKr2.1, in a voltageindependent manner [127]. Carbon monoxide also inhibits inward-rectifying potassium (Kir) channels, and prolongs the action potential duration [128, 129]. In their study, Ficker et al. demonstrated that, despite there being an increase in cardiac calcium current, there is reduced trafficking of cardiac potassium channel (hERG channels) to the cell surface among patients treated with arsenic trioxide, resulting in QT prolongation [130]. Another study revealed that arsenic trioxide blocks both $\mathrm{IKr}$ and $\mathrm{IKs}$ at clinically relevant concentrations. However, it also activates IK-ATP [131]. Graff et al. discovered that exposing rat cardiac myocytes to noncytotoxic concentrations of zinc and vanadium slowed the spontaneous beating rate [132].

In summary, the studies on the several components of cigarette smoke with known or suspected cardiotoxicity consistently show that they depress sodium and potassium 
channels, with some mixed effects on calcium channels, and a range of effects on ECG (Figure 1.3). Overall, the constituents appear to result in prolonged action potential duration from inhibition of sodium and potassium channels. Apart from formaldehyde, most other cigarette non-nicotine constituents have been shown to stimulate the cardiac sympathetic nervous system. With $>4000$ non-nicotine chemicals in cigarette smoke, and a complex mixture of effects on the sympathetic and parasympathetic pathways, the nonnicotine constituents can have a varied effect on surface ECG. Furthermore, most of the studies on the effects of non-nicotine constituents of cigarette smoke are derived from ex vivo or in vitro animal studies, with concentrations typically higher than those possibly encountered during cigarette and nicotine use. For instance, carbon monoxide causes tissue hypoxia, and, in addition to nicotine, has been implicated in promoting cardiac arrhythmias [133]; however, Benowitz et al. found that when carbon monoxide was administered under conditions similar to those of cigarette smoking, it had no significant effect on blood pressure, heart rate, plasma catecholamines, platelet aggregation, or C-reactive protein [134]. These results suggest that the short-term chronotropic, pro-thrombotic, and proinflammatory effects of smoking are probably due to components of cigarette smoke other than carbon monoxide. 


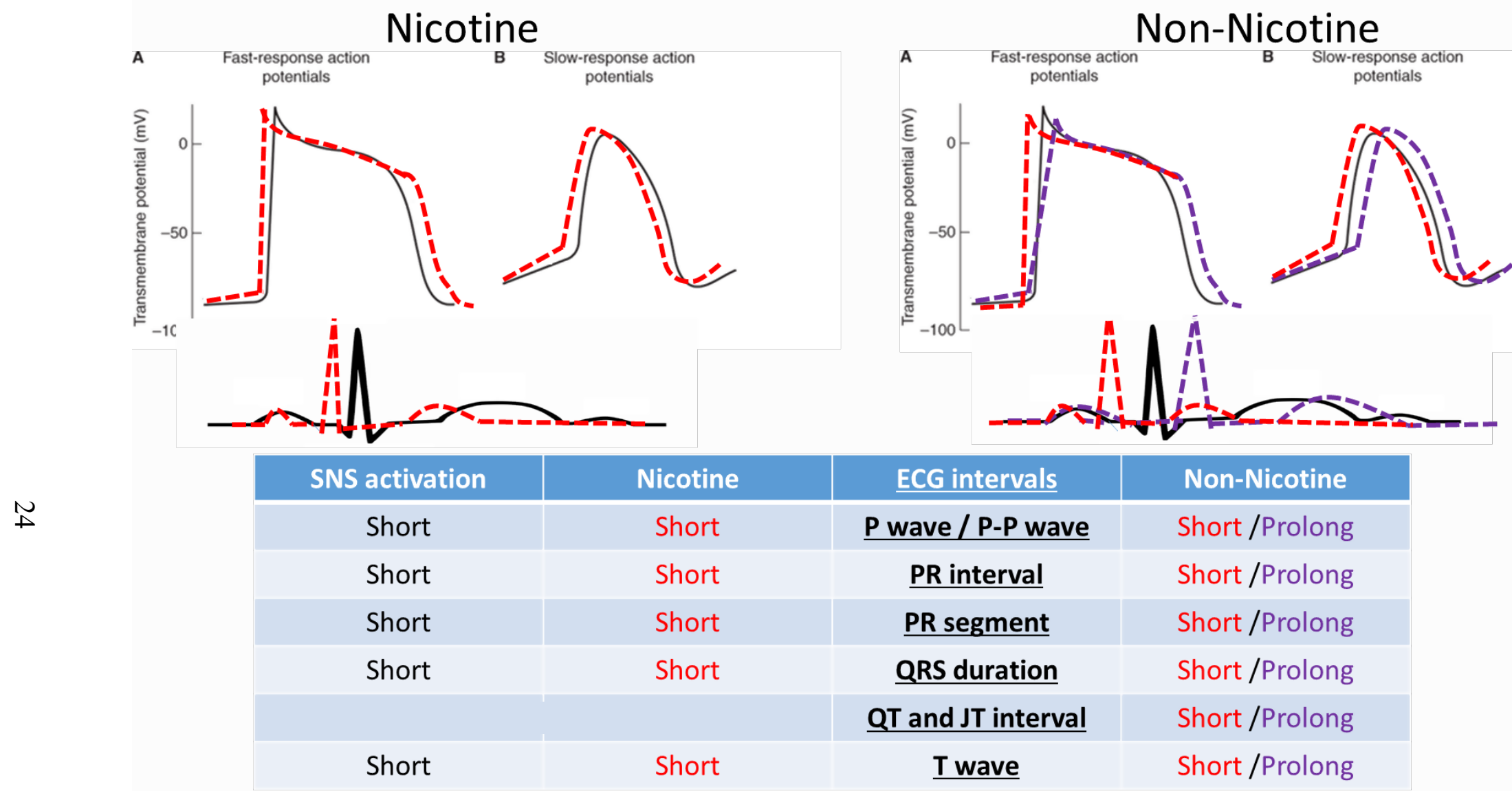

Figure 1.3 Direct effects of nicotine and non-nicotine constituents on cardiac ion channels and action potential. SNS - Sympathetic Nervous Activation 


\section{Indirect (systemic) effects}

There are several short- and long-term homeostatic mechanisms to ensure adequate blood flow, pressure, distribution, and perfusion, and are categorized into three groups: neural, humoral, and autoregulatory mechanisms. These mechanisms can also indirectly alter cardiac autonomic output and electrophysiology. Table 1.2 summarizes the stimuli, receptors, and physiologic effects most pertinent to cigarette smoking and nicotine-related changes in human autonomic reflexes.

Neural reflexes to tobacco exposure: The baroreflex feedback loop is one of the most important mechanisms controlling arterial pressure on a beat-to-beat basis. It achieves this through arterial baroreceptors located in the carotid sinus and aortic arch. These receptors are mechano-sensitive, and the distension of the vessels that occurs at each heart beat leads to action potential generation on peripheral nerves that transmit to the central nervous system, buffering arterial pressure fluctuations through changes in sympathetic and parasympathetic activity. Therefore, when blood pressure rises, the baroreceptor afferent tone increases, leading to increased vagal efferent activity and diminished sympathetic outflow. These effects will lead to a decrease in cardiac output by decreasing heart rate and cardiac contractility. Additionally, the fall in sympathetic tone to blood vessels, as well as increased vagal effect activity (through increased guanylyl cyclase and cGMP activity) leads to vasodilation and diminished vascular resistance. There is strong evidence that this crucial inhibitory role of the baroreflex arc is blunted in habitual smokers, and also impaired during acute exposure to smoking [72, 135, 136]. Grassi et al.'s study showed that there is sympathetic activation induced by smoking via increased release or a reduced clearance of catecholamines at the neuroeffector junctions. However, the central 
sympathetic activity is inhibited by smoking, presumably via baroreceptor stimulation triggered by a pressor response to smoking [90]. There is also evidence that nicotine possibly decreases the baroreceptor sensitivity [137]. Besides nicotine, there are several other cigarette components, mainly PM 2.5, that directly alter baroreflex responsiveness in smokers $[72,138]$. In one study, a one-time exposure to acrolein caused a decrease in the sensitivity of baroreflex and increased incidence of arrhythmia in rats [139].

The peripheral arterial chemoreceptors in the carotid and aortic bodies are stimulated by decreased arterial $\mathrm{PO} 2$, increased $\mathrm{PCO} 2$, and increased $\mathrm{H}+$ concentration. Their stimulation causes hyperventilation, as well as increases in sympathetic neural activity and the rate and volume of breathing; chronic arterial chemoreflex sensitization in smokers could also lead to sustained sympathetic activation. Arterial chemoreceptors are activated by hypoxia, and chronic smokers may be at risk to toxic effects of carbon monoxide in tobacco smoke. Perez et al. showed that acrolein-exposure-induced cardiovascular effects in rats (i.e., an increase in systolic, diastolic and mean arterial blood pressure during exposure, and a decrease in cardiac contractility one day after exposure) were prevented after a blockade of carotid body signal transduction. This suggests that acrolein-induced cardiovascular responses may be mediated by carotid body-triggered changes in autonomic tone [140]. However, to date, there is no evidence for augmented arterial chemoreceptor sensitivity in habitual smokers. Instead, nicotine does not affect chemoreflex sensitivity, as evidenced by unchanged minute ventilation, apnea duration, and oxygen saturation after nicotine and placebo in normoxia [141, 142].

Airways are lined with vagal afferent nerve fibers, including non-myelinated afferent $\mathrm{C}$-fibers sensitive to noxious chemicals. A subset of these vagal C-fibers expresses 
the TRP channels, which sense a variety of mechanical and chemical stimuli, such as mechanical stretch, sheer stress, oxidative stress, inflammation, and endogenous and exogenous chemicals. When exposed to irritants, TRP channels induce the local release of neuropeptides from cells, resulting in a local inflammatory response that reflexively increases efferent sympathetic nerve activity. This may cause further airway irritation and reflex responses, such as cough and reactive airway dysfunction [50, 143, 144]. However, there is little evidence to support whether smoking and its constituents directly activate TRP channels to modulate the human cardiac autonomic nervous system.

There are also respiratory-related changes in heart rate, specifically termed respiratory sinus arrhythmia, to help match pulmonary blood flow to lung inflation, and to maintain an appropriate diffusion gradient for oxygen in the lungs (heart rate increases during inspiration and decreases during the post-inspiration/expiration period) [145]. Consequently, different respiratory patterns during or after cigarette smoking may differentially affect cardiac autonomic function [146]. Cardiopulmonary receptors are found in low-pressure portions of the circulation, such as walls of the atria and pulmonary arteries. These mechano-sensitive receptors are activated by the distension of the vessel walls, responding to changes in central blood volume. The cardiopulmonary baroreflexes normally exert a tonic inhibitory influence on the SNS. However, cigarette smoking and nicotine product use in humans does not typically produce challenges that are extreme enough to affect these cardiopulmonary receptors or other similar innate reflexes that also alter cardiac electrophysiology (such as temperature, hypoxia, acidosis etc).

Autoregulation: Local, self-regulatory mechanisms, including chemical and myogenic controls, allow each region of tissue to adjust its blood flow, and thus its 
perfusion. Chemosensitive nerve endings are also found throughout the cardiovascular and respiratory systems, and are stimulated by several exogenous chemicals and endogenous chemicals formed and released in response to conditions such as hypoxia, ischemia, certain mechanical demands, inflammation, or toxin exposures. The efferent pathways of the reflex involve inhibition of sympathetic outflow to peripheral vessels and increased activity in efferent vagal fibers to the heart [50]. The myogenic response is a reflex to the stretching of the smooth muscle of the arteriolar walls as changes in blood flow occur through the vessel (e.g., vasoconstriction in response to increased intraluminal pressure). Increased peripheral vascular resistance, cigarette smoking, and nicotine also have detrimental effects on coronary microvascular function (e.g., increases in coronary flow velocity and resistance, and decreases in flow reserve) and can cause vascular dysfunction [147-151], possibly via $\beta$-adrenergic receptor [152].

Humoral to tobacco exposure: Several studies have suggested that humoral systems play a vital role in maintaining cardiac electric activity, and changes in their production or action pathways may contribute to various cardiac diseases. Beyond the neurotransmitters acetylcholine and norepinephrine, there is a local presence of peptidergic and nitrergic neurons along with their associated neurotransmitters, such as neuropeptide-Y, vasostatin, galactin, vasoactive intestinal peptide, nitric oxide synthase, and angiotensin-II [153, 154]. Neuropeptide-Y, coreleased by prolonged sympathetic activation, reduces acetylcholine release from the nearby vagal nerve ending, and it is an excellent example of sympathovagal cross-talk. These non-cholinergic, non-adrenergic neurotransmitters often exert effects similar to cholinergic or adrenergic agonists or antagonists [153]. The release of these neurotransmitters/modulators is often highly dependent on the level of neuronal 
stimulation, and they tend to be slowly diffusing molecules that often function as neuromodulators, rather than classical neurotransmitters.

Smoking has multiple effects on hormone secretion, some of which bear crucial clinical implications, and are mainly mediated by nicotine. Most acute data are from nicotine administration, whereas chronic data are predominantly from studies on cigarette smokers. Smoking affects pituitary, thyroid, adrenal, testicular and ovarian function, calcium metabolism, and the action of insulin differently in acute and chronic conditions $[155,156]$. In particular, the activation of nicotinic acetylcholine receptors in the adrenal medulla leads to increased circulating catecholamine levels with corresponding cardiovascular and metabolic responses. The renin-angiotensin-aldosterone system (RAAS) and adrenal gland is also activated by the hypothalamic-pituitary-adrenal axis $[155,156]$

Other mechanisms by which smoking, and nicotine can influence cardiac autonomic function are inflammation and oxidative stress. The role of autonomic function in regulating oxidative stress is supported by previous evidence that the increase in adrenergic drive may result in catecholamine excitotoxicity, increased oxidative stress, and free-radical myocardium injury. There are two main phases in cigarette smoke; particulate phase and gas phase [157]. The two phases are rich in free radicals, and non-radical oxidants. Therefore, acute and chronic exposure to smoking causes increased oxidative stress from direct damage by radical species and the inflammatory response, as well as through sympathetic neural activation [72, 158-160]. In one study, smoking decreased the overall $\alpha$ - and $\beta$-adrenergic receptor concentration almost immediately after tobacco smoke 
exposure in rats - perhaps through receptor desensitization resulting from a release of catecholamines - but was rapidly reversible after the termination of the exposure [161]. 
Table 1.2 Potential mechanisms of indirect effects of cigarette smoking on human cardiac electrophysiology

\begin{tabular}{|c|c|c|c|c|c|}
\hline System & Receptors & Stimulus & $\begin{array}{l}\text { Effect on } \\
\text { SNS }\end{array}$ & $\begin{array}{l}\text { Effect on } \\
\text { PNS }\end{array}$ & Studies on the effects of smoking / nicotine \\
\hline Baroreflex & $\begin{array}{l}\text { Carotid artery / } \\
\text { Aorta }\end{array}$ & $\begin{array}{l}\text { High Blood } \\
\text { Pressure }\end{array}$ & Decrease & Increase & $\begin{array}{l}\text { Activates the system by increasing blood } \\
\text { pressure through release of catecholamines } \\
\text { from end terminals. Smoking also impairs } \\
\text { the baroreflex system }\end{array}$ \\
\hline Chemoreflex & $\begin{array}{l}\text { Carotid / aortic } \\
\text { (peripheral), } \\
\text { medulla (central) }\end{array}$ & $\begin{array}{l}\text { Low } \mathrm{PaO} 2 \text {, High } \\
\mathrm{PaCO} 2 / \mathrm{pH}\end{array}$ & $\begin{array}{l}\text { Increase } \\
\text { (increase } \\
\text { pulmonary } \\
\text { ventilation) }\end{array}$ & - & $\begin{array}{l}\text { Nicotine does not increase chemoreflex } \\
\text { sensitivity to hypoxia. }\end{array}$ \\
\hline Temperature & CNS & High Temp & Increases & - & $\begin{array}{l}\text { Temperature increases in lungs; decreases in } \\
\text { skin temp }\end{array}$ \\
\hline $\begin{array}{l}\text { Inflammation / } \\
\text { Oxidative stress }\end{array}$ & $\begin{array}{l}\text { Lung afferent } C \\
\text { fibers, vascular, } \\
\text { myocardial }\end{array}$ & $\begin{array}{l}\text { Direct / Reactive } \\
\text { Oxygen Species }\end{array}$ & Increase & Decrease & $\begin{array}{l}\text { Increases inflammation and oxidative stress } \\
\text { during acute and chronic exposure }\end{array}$ \\
\hline Endocrine & $\begin{array}{l}\text { hypothalamic- } \\
\text { pituitary axis }\end{array}$ & & Increase & - & $\begin{array}{l}\text { Chronic: increases thyroid hormones, } \\
\text { cortisol, possibly testosterone and estradiol. } \\
\text { Decreases prolactin and growth hormone } \\
\text { Acute: Increases prolactin, cortisol, growth } \\
\text { hormone, vasopressin, endorphin, } \\
\text { neuropeptide Y }\end{array}$ \\
\hline Cardiopulmonary & Ventricles & $\begin{array}{l}\text { High SNS (low } \\
\text { vol LV) }\end{array}$ & Decrease & Increase & - \\
\hline Pulmonary & Pulmonary & $\begin{array}{l}\text { chemical stimuli, } \\
\text { inflammation }\end{array}$ & $?$ & Increase & - \\
\hline Bainbridge & Atria & High blood vol & Increase & Decrease & - \\
\hline
\end{tabular}




\section{Literature review of effects of autonomic nervous system and smoking on electrocardiogram}

\section{Autonomic nervous system}

Heart rate: The sinoatrial node, also referred to as the pacemaker of the heart, coordinates heart contractions. Located in the upper right atria wall, it generates impulses that result in cardiac contraction and determines the heart rate. The ANS predominantly determines the actual heart rate, but nonautonomic contributions (e.g., hypoxia and temperature via chemoreflex and baroreceptors pathways) also affect the intrinsic heart rate. The heart rate provides a static index of the net effects of autonomic input to the sinus node, but it does not reflect direct information about individual sympathetic or parasympathetic input. However, this simple measure has prognostic value, as reflected in population-based studies, in which high resting heart rate (net predominance of sympathetic influence) is associated with increased all-cause mortality, death from CVD, and sudden death [162-164]. The elevated heart rate may not only predict the outcome, but may also be an actual causal determinant of CVD through several different mechanisms [165].

Heart rate variability: Efferent sympathetic and vagal activities directed to the sinus node are characterized by a synchronous discharge with each cardiac cycle that can be modulated by central (vasomotor and respiratory centers) and peripheral (oscillation in arterial pressure and respiratory movements) oscillators. These oscillators generate rhythmic fluctuations in efferent neural discharge that manifest as short- and long-term oscillation in the heart period. Analysis of these rhythms may permit inferences on the state and function of (a) the central oscillators, (b) the sympathetic and vagal efferent activity, 
(c) humoral factors, and (d) the sinus node [166]. The oscillation in the intervals between consecutive heartbeats can be measured for indices of HRV, using either time domain approaches (based on statistical analysis of R-R intervals) or frequency domain approaches (spectral analysis of a sequence of R-R intervals) [166]. Large population studies have shown a higher risk of coronary artery disease, death, and cardiac mortality in individuals with decreased HRV (in both healthy populations and patients with cardiac disease) [167].

$\mathrm{P}$ wave: The $\mathrm{P}$ wave is the expression of atrial depolarization and intraatrial conduction. Electrocardiographic $\mathrm{P}$ wave indices consist of the $\mathrm{P}$ wave duration, morphology, and amplitude, and provide information about the atrial structure and function. A prolonged P-wave duration $(>120 \mathrm{~ms})$ is considered a marker of atrial cardiopathy, which, in chronic cardiac disease, is usually reflective if reduced atrial conduction related to architectural changes of atrial walls. P-wave duration is affected by autonomic tone. In general, both sympathetic stimulation and parasympathetic blockade shorten P-wave duration, whereas sympathetic blockade prolongs it [58]. P-wave terminal force in lead V1 (PTFV1) is defined as the value of the amplitude multiplied by the duration of the terminal's negative deflection of the P wave in lead V1 of a standard 12-lead ECG. $\mathrm{P}$-wave area (PWA) is the total geometric area under the P wave in the 12-lead ECG. It is usually represented by the product of the duration and peak amplitude of the P wave, and is measured in microvolt $\times$ milliseconds. Both of these P-wave indices, together with Pwave axis (net direction of electrical forces within the atria), are also markers of atrial cardiopathy $[168,169]$. Therefore, acute sympathetic activation may shorten P-wave duration, whereas chronic sympathetic activation may prolong P-wave duration from longterm structural atrial remodeling. The $\mathrm{P}$ amplitude, which is mostly governed by atrial 
pressures, may be increased in height (and in depth of PTFV1) in acute and chronic sympathetic stimulation.

PR interval: PR interval is the period of time from the start of the $\mathrm{P}$ wave (atrial depolarization) until the start of the QRS complex (ventricular depolarization). Therefore, the determinants of PR interval are atrial depolarization and the conduction time from the sinus node to the atrioventricular node, His bundle, and Purkinje fibers [170]. The duration of PR interval is normally between 120 and $200 \mathrm{~ms}$. In most cases, a prolonged PR interval ( $>200 \mathrm{~ms}$ ) is determined by conduction delay in the atrioventricular node. The ANS's acute effects on PR intervals are well known, considering that autonomic innervation influences the conduction through the atrioventricular node junction by modulating the refractory period [61, 171]. However, in the chronic state, autonomic-imbalance-induced atrial fibrosis may also cause PR interval prolongation by slowing atrial depolarization and dromotropy. Both short [26, 27, 29, 30] and prolonged [30, 172-174] PR intervals have been associated with adverse clinical outcomes (stroke, atrial fibrillation, and all-cause mortality).

QRS complex: The QRS complex represents the electrical impulse as it spreads through the ventricles, and depicts ventricular depolarization. Ventricular depolarization is also influenced by autonomic modulation of the heart. In humans, increased sympathetic tone by $\beta$-adrenergic stimulation shortens overall QRS duration [175], heterogeneity of ventricular activation during disease states, and bundle branch blocks (mechanical and electrical dyssynchrony) [176]. The QRS complex voltage reflects the viable left ventricular mass and can be increased (e.g., athlete's heart, hypertensive heart disease [177]) or decreased (post-myocardial infarction, infiltrative cardiomyopathies, etc. [178]) 
during different disease states by several mechanisms [179], and both carry critical clinical implications [180-182]. The QRS amplitudes can also potentially be affected by the cardiac ANS [183-185]. However, stimulation of the left stellate ganglion produces little or no change in conduction velocity in the Purkinje system, or in the pattern of epicardial depolarization, suggesting that cardiac sympathetic nerve stimulation does not result in significant changes in the sequence of ventricular excitation [61].

QT interval: The QT interval is a marker of ventricular depolarization (QRS duration) and repolarization (JT interval), and autonomic tone is the primary determinant of their duration $[65,186]$. Changes in autonomic tone may alter QT intervals both indirectly, by modulating basal heart rate, and directly, by affecting ventricular repolarization kinetics of myocardial cells through neural and receptor-mediated mechanisms $[53,54,57]$. The effect of sympathetic nervous activity on the QT has been demonstrated [187-190]; however, in their study, Cappato et al. did not find that sympathetic tone influenced QT significantly, but rather that the cholinergic system appeared to slow down ventricular refractoriness and QT interval [191].

T wave: The $T$ wave is the asymmetrical wave in the ECG that reflects ventricular repolarization; it comes after the QRS complex and typically lasts approximately $150 \mathrm{~ms}$. The difference between the peak of the $\mathrm{T}$ wave and the isoelectric level during the same heart cycle is defined as T-wave amplitude (TWA). Several studies have suggested that TWA decreases, and even may invert, with sympathetic stimulation; conversely, the additional role of the cholinergic system remains unclear [192]. The Tp-Te interval is an index of transmural dispersion of repolarization, a marker of ventricular arrhythmia vulnerability [193]. An increase in Tp-Te reflects increased sympathetic nerve activity 
[194], rather than the release of circulating norepinephrine [194]. However, more recently, the influence of the ANS on the TWA and Tp-Te interval has fallen under heavy scrutiny, and appears to be an unreliable index of myocardial sympathetic activity [195-197].

In summary, acute and chronic cardiac autonomic imbalance varies by phenotype (normal and diseased states), and influences several ECG parameters differently.

\section{Role of $\beta$-adrenergic receptors}

Physiologic doses of epinephrine alter electrophysiology through $\beta$-receptor activation, and manifest as an acceleration of atrioventricular nodal conduction and shortening of refractoriness in the atrium and ventricle [198]. In contrast, norepinephrine slows atrioventricular nodal conduction and lengthens the atrial and ventricular effective refractory periods [199]. Therefore, norepinephrine may counteract several of the electrophysiological effects of circulating epinephrine during physiologic degrees of stress. Furthermore, a-adrenergic stimulation by epinephrine in the presence of propranolol was found to prolong atrial and ventricular refractoriness [199].

Most studies on the effect of $\beta$-adrenergic receptors on cardiac electrophysiology have been performed with either propranolol, a non-selective $\beta$-blocker, or isoproterenol, a non-selective $\beta$-adrenoreceptor agonist. Propranolol decreases heart rate and prolongs atrioventricular nodal conduction [200], and has been shown to have no significant effect on intra-ventricular conduction. Shortening of action potential plateau was also not evident after treatment with propranolol in normal cat ventricular muscles [89]. In the sinoatrial node and left atrial appendage cells of the guinea-pig heart, propranolol antagonized the positive chronotropism of nicotine and norepinephrine [88]. Studies conducted on five 
human volunteers showed that intravenous propranolol followed by smoking significantly decreased cardiac output, and significantly increased blood pressure and systemic vascular resistance compared to smoking alone [201]. Similar effects were observed in another study among 10 participants [202]. Smoking alone increased cardiac output, mean arterial blood pressure, and decreased the calculated systemic vascular resistance [201]. The cardiac output increases were due to a fall in systemic vascular resistance. The effect is exaggerated after propranolol, potentially via the inhibition of $\beta$-adrenergic receptors, either by preventing the vasodilatory effect of $\beta-2$ adrenergic stimulation, or as increased availability of $\alpha$-receptors may result in unopposed pronounced effects of vasoconstriction from epinephrine. However, another study was conducted using $80 \mathrm{mg}$ of oral propranolol on six volunteers, and there was no significant difference from the placebo in blood pressure or forearm hemodynamics, and no prevention of the acute vascular effects of cigarette smoking with $\beta$-blocker pre-treatment [203]. $\beta$-blockers are first line therapy for patients with Long QT syndrome (LQTS) — a genetic disorder that can potentially cause life-threatening cardiac arrhythmia, is characterized by delayed myocardial repolarization, and manifests as QT prolongation [204]. The response to $\beta$-blockers and epinephrine depends on the genotype $[204,205]$. Therefore, the differences in $\beta$-adrenergic receptormediated effects on ventricular repolarization are likely related to the stimulus (e.g., frequency of cigarette smoking), dose (route) of propranolol use, and the underlying genotype. Furthermore, the plasma propranolol steady-state concentration is lower among smokers compared to non-smokers, possibly via an increased rate of drug metabolism [206]. 
Besides $\beta$-adrenergic receptors and muscarinic receptors from the cholinergic system, there are several other receptors ( $\alpha$-adrenergic receptors, dopamine receptors, and adenosine receptors) found throughout the myocardium, with critical clinical effects on ECG (Table 1.3). However, the impacts of smoking and nicotine on the ECG via receptors other than $\beta$-adrenergic receptors are not well defined.

Table 1.3 Effects of cardiac receptors on chronotropy, dromotropy and inotropy

\begin{tabular}{lcccc}
\hline Neurotransmitter & Receptors & $\begin{array}{c}\text { Chronotropy } \\
\text { (SA node) }\end{array}$ & $\begin{array}{c}\text { Dromotropy } \\
\text { (AV node) }\end{array}$ & $\begin{array}{c}\text { Inotropy } \\
\text { (Ventricle) }\end{array}$ \\
\hline \multirow{2}{*}{ Norepinephrine } & $\alpha 1$ & + & + & + \\
& $\beta 1$ & + & + & + \\
Dopamine & D1, D2, D3, D5 & + & + & + \\
Acetylcholine & M2 & - & - & $?$ \\
\hline
\end{tabular}

Acute and chronic exposure from smoking

Chronic exposure to cigarette smoke (nicotine and non-nicotine constituents)

Due to the injurious and addictive nature of smoking, it is not ethical to perform an experimental study to investigate the chronic effects of smoking and nicotine-containing products in humans. Therefore, studies can best describe associations of outcomes (biomarkers of CVD and risk) from occupational exposures or chronic smoking. Occupational exposure to polycyclic aromatic hydrocarbons results in decreased HRV [207]. A similar decrease in parasympathetic activity has been observed in workers exposed to organic solvents (n-hexane, xylene, and toluene) [208]. Sub-chronic and longterm exposure to ambient fine particulate matter $\left(\mathrm{PM}_{2.5}\right)$ at concentrations several orders of magnitude lower than those in cigarette smoke has been associated with increased QTc 
duration in an elderly cohort [209]. Among 34 middle-aged individuals with metabolic syndrome, QRS and QTc intervals increased and HRV decreased $2 \mathrm{~h}$ after exposure to concentrated ambient ultrafine particles [210].

Table 1.4 A (atrial electrocardiographic indices) and B (ventricular electrocardiographic indices) summarizes the major human studies on the chronic effects of smoking. Overall, most large studies (sample size of greater than 100 smokers) consistently show that PR interval is decreased among chronic smokers compared to nonsmokers. We were not able to find any study that directly investigated the effect of smoking on the PR segment. In a prospective study of 60 smokers, Varenicline (partial agonist at the $\alpha 4 \beta 2$ nicotinic acetylcholine receptor) treatment was associated with a near-significant attenuation in PR interval (varenicline: $163.5 \pm 18.3 \mathrm{~ms}$, vs. placebo: $168.2 \pm 17.9 \mathrm{~ms} ; P$ $=.053$ ). However, RR interval, QT interval, and QTd were not significantly altered [211]. Two studies showed shorter P duration but increased P amplitude among smokers [212, 213]. The effects of smoking on QRS duration were the most varied. Most studies consistently showed that there was no significant difference in QT interval between smokers and non-smokers. Two studies found that the ST segment was decreased in chronic smokers versus non-smokers $[214,215]$. Few studies have found that the Tp-Te interval, Tpe/QT ratio, and Tpe/QTc ratio were higher among smokers [216, 217], even after varenicline administration [218].

Apart from the Zhang et al. study [219], none of these studies featured a quantitative method of smoking burden, such as cotinine, or an adjustment for clinically important covariates known to affect the baseline ECG (such as age, gender, body mass index, diabetes, coronary artery disease, etc). 
Table 1.4 Major human studies on the chronic effects of smoking on atrial (A) and ventricular (B) electrocardiographic indices The effect mentioned of the ECG parameters is among smokers compared to non-smokers are mentioned.

A) Atrial electrocardiographic indices

\begin{tabular}{|c|c|c|c|c|c|c|}
\hline Ref & Author, Year & $\begin{array}{l}\text { Chronic } \\
\text { Smokers }\end{array}$ & $\begin{array}{c}\text { Non- } \\
\text { smokers }\end{array}$ & $\mathrm{P}$ wave duration & PR interval & PR segment \\
\hline$[220]$ & Goldenberg, 2006 & 98 & 619 & - & Shorter & - \\
\hline$[221]$ & Chatterjee, 1989 & 224 & 232 & - & Shorter & - \\
\hline [212] & Sharma, 2017 & 150 & 50 & Shorter & Shorter & - \\
\hline$[222]$ & Baden, 1982 & 208 & 291 & - & Shorter & - \\
\hline$[223]$ & Khan, 2011 & 75 & 30 & - & Shorter & - \\
\hline [213] & Swathi, 2015* & 200 & 200 & Shorter & No difference & - \\
\hline$[214]$ & Sandhya, 2015 & 64 & 63 & - & No difference & - \\
\hline$[224]$ & Karapinar, 2010 & 30 & 30 & No difference & - & - \\
\hline$[225]$ & Goette, 2007 & 46 & 49 & No difference & - & - \\
\hline$[226]$ & Siddiqui, 2013 & 30 & 30 & No difference & No difference & - \\
\hline [227] & Venkatesh, 2010 & 50 & 50 & No difference & No difference & - \\
\hline
\end{tabular}


B) Ventricular electrocardiographic indices

\begin{tabular}{|c|c|c|c|c|c|c|}
\hline Ref & Author, Year & $\begin{array}{l}\text { Chronic } \\
\text { Smokers }\end{array}$ & $\begin{array}{c}\text { Non- } \\
\text { smokers }\end{array}$ & QRS duration & cQT / QT interval & cJT interval \\
\hline [226] & Siddiqui, 2013 & 30 & 30 & Shorter & No difference & - \\
\hline [213] & Swathi, 2015* & 200 & 200 & Shorter & No difference & - \\
\hline [227] & Vanketash, 2010 & 50 & 50 & Shorter & No difference & - \\
\hline [214] & Sandhya, 2015 & 64 & 63 & Shorter & No difference & Shorter ST \\
\hline [223] & Khan, 2011 & 75 & 30 & No difference & - & - \\
\hline$[222]$ & Baden, 1982 & 291 & 208 & No difference & No difference & - \\
\hline$[215]$ & Devi, 2013 & 44 & 44 & No difference & No difference & Shorter ST \\
\hline [219] & Zhang, 2011 & 3306 & 2242 & - & $\begin{array}{l}\text { No difference (adjusted } \\
\text { and used cotinine) }\end{array}$ & - \\
\hline [216] & İlgenli, 2015 & 24 & 23 & - & No difference & - \\
\hline$[228]$ & Karjalainen, 1997 & \multicolumn{2}{|c|}{10,717} & - & Decreased & - \\
\hline [229] & Ileri, 2011 & 30 & 30 & - & Increased & - \\
\hline [212] & Sharma, 2017 & 150 & 50 & No difference & Increased & - \\
\hline
\end{tabular}

* There were no statistical analyses performed. 
Acute exposure to smoking (nicotine and non-nicotine constituents)

Apart from the case reports, the scarce literature on the acute effects of individual constituents of cigarette smoking on human in vivo ECG has mainly investigated nicotine and carbon monoxide. Acute carbon monoxide intoxication in children impaired ventricular repolarization with higher mean heart rate, QT dispersion, QTc dispersion, P dispersion, $\mathrm{Tp}-\mathrm{Te}$, QTc durations, $\mathrm{Tp}-\mathrm{Te}$ dispersion, and $\mathrm{Tp}-\mathrm{Te} / \mathrm{QT}$ ratio compared to controls [230, 231]. However, it increased heart rate, ST-T wave changes, QTc dispersion, and TpTe dispersion in adults $[232,233]$. There is also long-term increased risk of acute myocardial infarction in carbon monoxide poisoning [234]. Among 16 smokers undergoing electrophysiological study, exposure to cigarette smoke produced a statistically significant shortening of the sinus cycle length after chemical denervation of the sinus node with $\beta$-blockers and atropine [235]. In a single case report of mild nicotine toxicity, a young smoker presented with a typ1 Brugada pattern, raising the possibility of sodium channel blockade [236]. Nicotine has proved to be arrhythmogenic in animals and cell preparations; however, despite some case reports linking nicotine to atrial fibrillation in humans, these findings have not been confirmed in pharmacological studies of nicotine administered to human subjects [133].

There are very few human experimental studies that have investigated the acute effects of smoking on the ECG. In their analysis of 40 healthy male participants (20 smokers), Akbarzadeh et al. discovered that heart rate, mean QT, and QTc dispersion increased 10 minutes after smoking a single cigarette [237]. In another study, 31 male smokers with atypical chest pain were screened with a treadmill exercise test, which revealed that the heart rate increased within 10 minutes and returned to baseline after 30 
minutes. Smoking was also associated with increased ectopic beats in these patients on the 24-hour Holter monitor [238]. On a signal averaged ECG, there was a minor lengthening of the filtered QRS duration among 15 long-term smokers after smoking two cigarettes [239].

To date, the most comprehensive assessment of acute smoking's impact on myocardial conduction was performed among 28 habitual smokers by Soad Bekheit and Evan Fletcher in 1976 using His bundle electrograms [240]. The following measurements were made from His bundle recordings in the control and "nicotine tracings" (immediately after the first puff, sequential atrial pacing at identical rates to the control tracings): intraatrial conduction time (P1A), A-V nodal conduction time (AH), His-Purkinje-system conduction time (HV), and total intraventricular conduction time (HS). They found that a few puffs of a cigarette increase the velocity of conduction and shortens the effective refractory period of the A-V node, whereas the conduction velocity in the His-Purkinje system is not affected [240]. However, these effects were not studied against those from nicotine exposure.

Acute cigarette smoking increases efferent sympathetic nerve activity, primarily via the release of the catecholamines norepinephrine and epinephrine (Table 4). This catecholamine release increases myocardial work and oxygen consumption through an increase in blood pressure, heart rate, and myocardial contractility [19]. Overall, the vast majority of published evidence suggests that acute and chronic active and passive exposure to cigarette smoke generates marked disruptions in normal autonomic function characterized by increased SNS drive, reduced PNS modulation, and overall HRV. This phenomenon is partly attributable to a nicotine-induced up-regulation of catecholamine 
release, generating potent acute and chronic effects on cardiovascular regulation through SNS activation [241].

\section{$\underline{\text { Hypothesis }}$}

Research Hypothesis:

Nicotine in cigarette smoke alters myocardial conduction through $\beta$-adrenergic receptors

\section{Alternate Hypothesis:}

Non-nicotine constituents in cigarette smoke alter myocardial conduction though $\beta$ adrenergic receptors and non- $\beta$-adrenergic pathways

Central hypothesis:

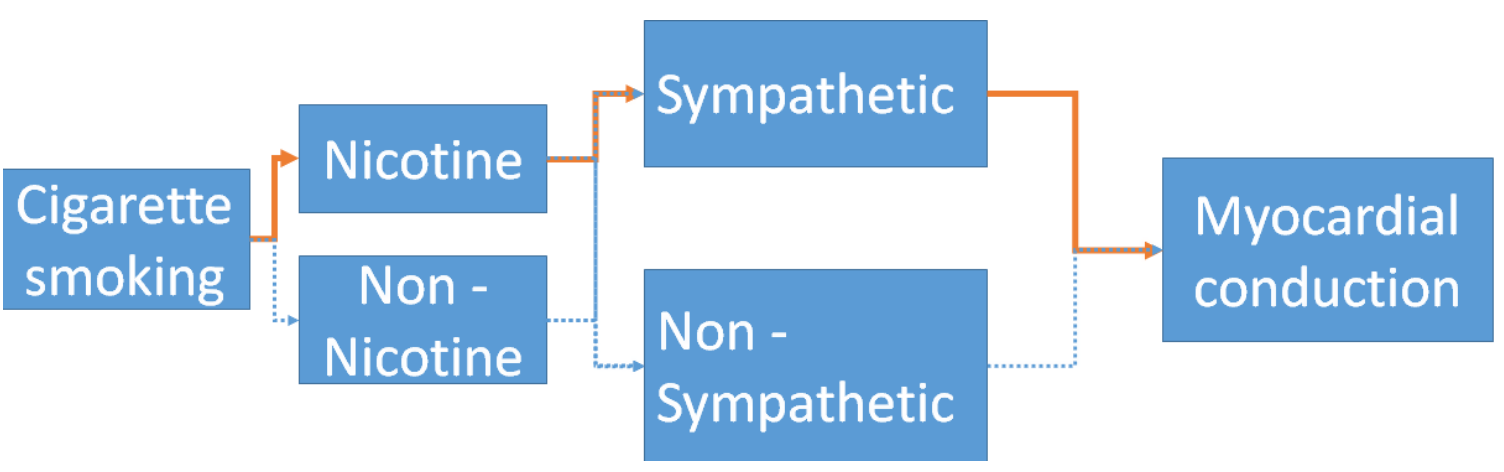

Aim 1 Explore the relationship between cigarette smoking and properties of myocardial conduction.

Aim 2 Test the influence of catecholamines in the association between smoking and myocardial conduction.

Aim 3 Acute effects of cigarette smoking and nicotine with and without $\beta$-blocker on electrocardiogram. 


\section{Ethical considerations}

The primary therapeutic use of nicotine is in treating nicotine dependence. Controlled levels of nicotine are given to patients through gum, dermal patches, lozenges, inhalers, or nasal and oral sprays to wean them off their dependence, increasing quitting success by $50 \%-70 \%$ [242]. In contrast to recreational nicotine products, which have been designed to maximize the likelihood of addiction, nicotine replacement products are designed to minimize addictiveness. Hence, physiological studies on the effects of chronic use of pure nicotine on humans are accompanied by the concern for potential addiction among non-smokers, or the tolerance or modulation of neural plasticity among habitual smokers. It is for this reason that there are no robust human physiological studies investigating the cause-effect relationship of prolonged exposure to pure nicotine. There are some studies on prolonged nicotine replacement therapies in smokers who have quit smoking, and in these studies, no adverse effects have been found when nicotine medication was administered for months to several years. Given the highly addictive nature of nicotine, these studies are more appropriately termed as acute or chronic exposure of nicotine in chronic tobacco (nicotine) users. Therefore, to investigate the chronic effects of tobacco use on the electrocardiogram, we analyzed plasma/urine cotinine, a highly sensitive and specific marker of chronic nicotine exposure, as well as urinary catecholamines, in order to investigate the relationship between the sympathetic nervous system, smoking, and the electrocardiogram. Furthermore, to study the acute effects of smoking and nicotine with and without a $\beta$-blocker, we designed an open-label $2 \times 2$ factorial experimental trial. 


\section{CHAPTER II}

\section{CHRONIC EFFECTS OF CIGARETTE SMOKING ON ELECTROCARDIOGRAM}

\section{Cigarette smoking, ECG and interaction with Atrioventricular nodal blockers}

Aim 1 Explore the relationship between cigarette smoking and properties of myocardial conduction

\section{Participants from NHANES database}

The availability of serum cotinine levels and digital ECG in the Third National Health and Nutrition Examination Survey (NHANES-III) provides a unique opportunity to examine the association between tobacco exposure and ECG parameters, in a large nationally representative human population. The NHANES is a program of studies designed to assess the health and nutritional status of adults and children in the United States. The survey is unique in that it combines interviews and physical examinations. The survey examines a nationally representative sample of about 5,000 persons each year. These persons are located in counties across the country, 15 of which are visited each year. The NHANES interview includes demographic, socioeconomic, dietary, and health-related questions. The examination component consists of medical, dental, and physiological measurements, as well as laboratory tests administered by highly trained medical personnel. To produce reliable statistics, NHANES over-samples persons 60 and older, African Americans, and Hispanics. All participants visit the physician and all (but the very young) have a blood sample taken. Health interviews are conducted in respondents' homes. Health measurements are performed in specially-designed and equipped mobile centers, which travel to locations throughout the country [243]. 
Complete details about NHANES survey components, survey methodology, and sampling procedures are available from the Centers for Disease Control NHANES website [244]. Briefly, NHANES data are not obtained using a simple random sample. Rather, a complex, multistage, probability sampling design is used to select participants representative of the civilian, non-institutionalized US population. The sample does not include persons residing in nursing homes, members of the armed forces, institutionalized persons, or U.S. nationals living abroad. A sample weight is assigned to each sample person. It is a measure of the number of people in the population represented by that sample person in NHANES, reflecting the unequal probability of selection, nonresponse adjustment, and adjustment to independent population controls. NHANES is designed to sample larger numbers of certain subgroups of particular public health interest. Oversampling is done to increase the reliability and precision of estimates of health status indicators for these population subgroups.

\section{Measures}

During the NHANES, individuals are asked questions related to smoking status, duration, and smoking-related behaviors. Smoking status was assessed in the home, by trained interviewers using the Computer-Assisted Personal Interviewing System (CAPI). Participants responded to whether they currently smoke cigarettes daily, some days, or not at all. Participants were categorized as never-smokers (individuals who have smoked $<100 \mathrm{cig} /$ lifetime), former smokers (having smoked $>100 \mathrm{cig} /$ lifetime but do not currently smoke), and current smokers. Current smokers are further classified as daily smokers 
(smoking cigarettes every day) and nondaily smokers (identifies as a smoker, but does not smoke cigarettes every day).

\section{Cotinine}

Measures such as cigarettes per day are imprecise indicators of tobacco smoke exposure because of variability in how smokers smoke their cigarettes. There is considerable individual variability in smoke intake, even by people smoking the same brand of cigarettes, and the cigarette design and how the cigarette is smoked influence toxic exposures. Therefore, the optimal assessment of exposure to tobacco smoke is the analysis of biomarkers for quantifying the systemic exposure of smokers to toxic constituents of smoke derived from tobacco use [245]. Nicotine measurement is highly specific for tobacco use or exposure (in the absence of nicotine medication use), but because of nicotine's short half-life $(2 \mathrm{~h})$ the method is not recommended for general use. Nicotine is extensively metabolized to a number of metabolites by the liver, of which quantitatively, the most important metabolite of nicotine is the lactam derivative, cotinine. In humans, about $70-80 \%$ of nicotine is converted to cotinine. Cotinine is a highly specific and sensitive marker for tobacco use (in the absence nicotine medication use) and has the advantages of a fairly long half-life (16 h) [245]. Measuring cotinine in people's blood is the most reliable way to determine exposure to nicotine for a marker for both active smoking, and as an index to Environmental Tobacco Smoke (ETS) exposure, or "passive smoking". There is a high correlation among cotinine concentrations measured in plasma, saliva, and urine, and measurements in any one of these fluids can be used as a marker of nicotine intake. Cotinine concentrations tend to be higher $(3-8 x)$ in urine than in serum; 
however, for studies requiring a quantitative assessment of exposure, plasma or serum is regarded as the fluid of choice. Therefore, serum is used for cotinine measurement in NHANES [243].

Serum cotinine is measured by an isotope dilution-high performance liquid chromatography / atmospheric pressure chemical ionization tandem mass spectrometry (ID HPLC-APCI MS/MS). Briefly, the serum sample is spiked with methyl-D3 cotinine as an internal standard, and after an equilibration period, the sample is applied to a basified solidphase extraction column. Cotinine is extracted off the column with methylene chloride, the organic extract is concentrated, and the residue is injected onto a short, C18 HPLC column. The eluant from these injections is monitored by APCI-MS/MS, and the $\mathrm{m} / \mathrm{z} 80$ daughter ion from the m/z 177 quasi-molecular ion is quantitated, along with additional ions for the internal standard, external standard, and for confirmation. Cotinine concentrations are derived from the ratio of native to labeled cotinine in the sample, by comparisons to a standard curve [243].

Electrocardiogram: Participants aged $\geqq 40$ years who attended the medical examination received a resting 12-lead ECG with a Marquette MAC 12-unit (Marquette Electronics, Inc., USA), and analyzed using the NOVACODE ECG program, which classified the ECGs as per the Minnesota Coding (MC) System. Details of the ECG examination have been published previously [244]. Computerized automated analysis of the electrocardiographic data was performed with visual inspection of outlier values by a trained technician in a central ECG core laboratory (EPICARE Center at the Wake Forest School of Medicine, Winston Salem, NC). PR interval and P duration in lead II and global QRS duration and QT interval were automatically measured. PR segment was calculated 
as the difference between PR interval and P duration. QT interval was Heart rate corrected using the Framingham formula (QTc), calculated as QT $+154 \times(1-60 / \mathrm{HR})$ [246]. To measure the association between cotinine and the ECG intervals (PR interval, P duration, PR segment, QRS, QTc and JT) we divided them into three groups; >95th percentile (long), 5 - 95th percentile (reference) and $<5$ th percentile (short). For the purpose of this analysis, we only included NHANES III participants who had good quality ECG recording and with no major ECG abnormalities including electrocardiographic evidence of myocardial infarction or ischemia as defined by Minnesota Electrocardiogram Classification, and available serum cotinine data, medical history, medication use, and anthropometric measurements.

Covariates definitions: Since several ECG characteristics significantly differ by demographics, anthropometric features and cardiovascular risk factors and cardiac medications [247-252], we identified clinically important covariates to adjust for their effect on ECG parameters. Diabetes was defined as a fasting plasma glucose level of $\geq 126$ $\mathrm{mg} / \mathrm{dl}$, glycosylated hemoglobin $\mathrm{A} 1 \mathrm{C}$ values $\geq 6.5$, or a history of glucose-lowering medications. Hypertension was defined as systolic blood pressure of $\geq 130 \mathrm{~mm} \mathrm{Hg}$, diastolic blood pressure of $\geq 80 \mathrm{~mm} \mathrm{Hg}$, or use of blood pressure-lowering medications. Body mass index was computed as the weight in kilogram divided by the square of the height in meter, and obesity was defined as a body mass index of $>30 \mathrm{~kg} / \mathrm{m} 2$. Age, gender, race/ ethnicity, and smoking status were self-reported. Chronic Obstructive Pulmonary Disease (COPD) was defined as patients with a combination of asthma and emphysema. Alcohol consumption was assessed by the food frequency questionnaire. Participants reported the number of times that they drank beer, wine, and hard liquor in the past month, 
and we categorized total alcohol consumption into 4 groups $(0,1-4,5-13, \geq 14$ drinks/month). Serum cotinine levels $>15 \mathrm{ng} / \mathrm{ml}$ were used to categorize the participants as smokers, while those $\leq 15 \mathrm{ng} / \mathrm{ml}$ were categorized as non-smokers .

\section{Statistical analyses}

The ECG sampling weights were used in the analysis to account for the complex sampling design [253]. Categorical variables were reported as frequency and population percents, whereas continuous variables were recorded as geometric mean \pm standard error for all demographic tables. Statistical significance in demographic tables was tested using survey weighted analysis; for continuous variables, $t$ test or ANOVA, whereas Rao-Scott chi-square was used for categorical variables. Survey weighted Multinomial regression was used to calculate the odds ratios and $95 \%$ confidence intervals for the association between serum cotinine levels $(>15 \mathrm{ng} / \mathrm{ml})$ and ECG intervals using the group $5-95$ th percentile as reference. A sensitivity analyses was carried out, and the overall results did not differ after excluding those participants who had serum cotinine $>15 \mathrm{ng} / \mathrm{ml}$, and identified themselves as never-smokers $(n=134)$ or ex-smokers $(n=216)$. Multivariable adjusted models were constructed with incremental adjustments as follows: model 1 adjusted for age, sex and race-ethnicity; and model 2 adjusted for model 1 covariates and heart rate, obesity, diabetes, hypertension, dyslipidemia, previous CVD, congestive heart failure, COPD and alcohol intake; and model 3 adjusted for model 2 covariates and $\beta$ blockers, calcium channel blockers and anti-arrhythmic drugs. Model 4 was created based only on the baseline characteristics associated with the ECG intervals with $\mathrm{p}<0.10$ (Supplementary 2.1.2-2.1.7). The heart rate was not included in the model for QTc. We 
also performed fully adjusted survey weighted linear regression between continuous cotinine levels and ECG intervals as continuous variables. We conducted subgroup analyses stratified by age (cut-off point by median - 59 years) and gender. A 2-sided p value of $\leq 0.05$ was considered significant for main effects and for interactions. Data were analyzed using the survey procedures in SAS, version 9.4 (SAS Institute, North Carolina).

\section{Results}

A total of 5,633 study participants (mean age $59 \pm 13$ years, $53 \%$ women, $48 \%$ nonHispanic white) were included in this analysis. A total of 1,580 (28\%) participants were identified as smokers (serum cotinine levels $>15 \mathrm{ng} / \mathrm{ml}$ ). Smokers were more likely to be younger in age, men, with lower prevalence of dyslipidemia and $\beta$-blocker use and higher prevalence of COPD, higher resting heart rate and higher alcohol intake (Table 2.1.1). The 5th and 95th percentiles of ECG variables are shown in Supplementary Table 2.1.1. and Supplementary Tables 2.1.2-2.1.7 represent the geometric mean and standard error for ECG variables, and represent the population percentages for categorical baseline characteristics. 
Table 2.1.1 Baseline Participants Characteristics (Total N=5,653)

\begin{tabular}{|c|c|c|c|}
\hline \multirow[b]{2}{*}{ Characteristic } & \multicolumn{2}{|c|}{ Smoker } & \multirow[b]{2}{*}{ p-value } \\
\hline & $\begin{array}{c}\text { No } \\
\leq 15 \mathrm{ng} / \mathrm{ml} \\
\mathrm{n}=4073,72 \%\end{array}$ & $\begin{array}{c}\text { Yes } \\
>15 \mathrm{ng} / \mathrm{ml} \\
\mathrm{n}=1580,28 \%\end{array}$ & \\
\hline Age (years)* & $56.4 \pm 0.44$ & $53.4 \pm 0.45$ & $<0.001$ \\
\hline Women & $2371(59.1 \%)$ & $629(43.7 \%)$ & $<0.001$ \\
\hline Non-Hispanic White & $2122(82.2 \%)$ & $738(80.8 \%)$ & 0.291 \\
\hline \multicolumn{4}{|l|}{ Smoking status } \\
\hline Never & $2381(56.1 \%)$ & $134(5.8 \%)$ & \multirow{3}{*}{$<0.001$} \\
\hline Current & $67(1.6 \%)$ & $1230(78.4 \%)$ & \\
\hline Past & $1625(42.3 \%)$ & $216(15.8 \%)$ & \\
\hline Diabetes mellitus & $479(7.5 \%)$ & $143(7.0 \%)$ & 0.630 \\
\hline Hypertension & $1427(31.8 \%)$ & $489(28.8 \%)$ & 0.167 \\
\hline Dyslipidemia & $1066(29.3 \%)$ & $308(23.1 \%)$ & 0.004 \\
\hline Obesity & $853(18.0 \%)$ & $233(15.3 \%)$ & 0.120 \\
\hline COPD & $263(6.8 \%)$ & $160(10.8 \%)$ & $<0.001$ \\
\hline Heart rate (beats/minute) & $67.6 \pm 0.3$ & $68.9 \pm .04$ & 0.012 \\
\hline Prior cardiovascular disease & $172(3.2 \%)$ & $67(3.5 \%)$ & 0.698 \\
\hline Congestive Heart Failure & $131(1.8 \%)$ & $54(1.7 \%)$ & 0.872 \\
\hline \multicolumn{4}{|l|}{ Alcohol drinks per month } \\
\hline 0 & $2415(50.7 \%)$ & $762(45.1 \%)$ & \multirow{4}{*}{0.003} \\
\hline $1-4$ & $670(18.0 \%)$ & $253(17.2 \%)$ & \\
\hline $5-13$ & $440(13.9 \%)$ & $200(13.3 \%)$ & \\
\hline$>13$ & $539(17.4 \%)$ & $359(24.4 \%)$ & \\
\hline B blockers & $320(8.2 \%)$ & $87(5.9 \%)$ & 0.009 \\
\hline Calcium channel blockers & $333(6.7 \%)$ & $103(6.0 \%)$ & 0.453 \\
\hline Antiarrhythmic drugs & $34(0.6 \%)$ & $17(1.5 \%)$ & 0.129 \\
\hline
\end{tabular}

*Except for age (which is represented by geometric mean and standard deviation), all other variables are represented as frequency and column percentages. 
PR interval, $P$ wave and $P R$ segment

In multinomial logistic regression models adjusted for demographics, smoking was associated with increased odds of short PR interval and short PR segment ( $p$-value $<0.01)$ (Table 1.2). This association was not attenuated after further adjustment for CVD risk factors and AV nodal blockers, and was consistent in subgroups stratified by age and sex (Supplementary Table 2.1.8). No significant association was observed with smoking status and $\mathrm{P}$ wave duration. Follow-up linear regression analyses revealed a significant negative association between continuous cotinine levels and PR segment, but not with P wave and PR interval. According to model 4, the PR segment shortened by $0.554 \mathrm{~ms}$ per $100 \mathrm{ng} / \mathrm{ml}$ increase in cotinine levels (Table 2.1.3).

\section{QTC interval, JT interval and QRS duration}

In fully adjusted multinomial regression models, serum cotinine was associated with short QRS and long JT (p-value $<0.01$ ), but not with abnormal QTc (Table 2.1.4). The association between smoking status and short QRS duration $\left(<5^{\text {th }}\right.$ percentile $)$ and long JT interval $\left(>95^{\text {th }}\right.$ percentile) was consistent in subgroups stratified by age and sex (Supplementary Table 2.1.8). However, linear regression showed a significant association overall between continuous cotinine levels and QTc, but not with QRS duration and JT interval (Table 2.1.5). According to model 4, QTc shortened by $-1.345 \mathrm{~ms}$ with every $100 \mathrm{ng} / \mathrm{ml}$ increase in cotinine.

The effect of smoking status on baseline ECG (results from logistic regression) is shown in Figure 2.1.1, and the relationship between cotinine and ECG (results from linear regression) is shown in Figure 2.1.2. 
Table 2.1.2 Association between smoking status and abnormal PR interval and its components

ECG variables groups: Short $\left(<5^{\text {th }}\right.$ percentile $)$, reference $\left(5-95^{\text {th }}\right.$ percentile $)$ and Long ( $>95^{\text {th }}$ percentile). $\mathrm{N}=5,653$

\begin{tabular}{|c|c|c|c|c|c|c|c|}
\hline & & \multicolumn{2}{|c|}{ PR interval } & \multicolumn{2}{|c|}{$\mathrm{P}$ duration } & \multicolumn{2}{|c|}{ PR segment } \\
\hline & & $\begin{array}{l}\text { Odds ratio } \\
(95 \% \mathrm{CI})\end{array}$ & $\begin{array}{c}\mathrm{p}- \\
\text { value }\end{array}$ & $\begin{array}{l}\text { Odds ratio } \\
(95 \% \mathrm{CI})\end{array}$ & $\begin{array}{c}\mathrm{p}- \\
\text { value }\end{array}$ & $\begin{array}{l}\text { Odds ratio } \\
(95 \% \mathrm{CI})\end{array}$ & $\begin{array}{c}\mathrm{p}- \\
\text { value }\end{array}$ \\
\hline \multirow{3}{*}{ Model 1* } & Short & $\begin{array}{c}1.63 \\
(1.07,2.48)\end{array}$ & 0.024 & $\begin{array}{c}1.07 \\
(0.72,1.57)\end{array}$ & 0.746 & $\begin{array}{c}1.99 \\
(1.30,3.05)\end{array}$ & 0.002 \\
\hline & Reference & - & & - & & - & \\
\hline & Long & $\begin{array}{c}0.73 \\
(0.46,1.16)\end{array}$ & 0.186 & $\begin{array}{c}0.88 \\
(0.55,1.40)\end{array}$ & 0.585 & $\begin{array}{c}0.93 \\
(0.64,1.37)\end{array}$ & 0.726 \\
\hline \multirow{3}{*}{ Model $2^{\dagger}$} & Short & $\begin{array}{c}1.57 \\
(1.02,2.43)\end{array}$ & 0.042 & $\begin{array}{c}1.10 \\
(0.75,1.60)\end{array}$ & 0.639 & $\begin{array}{c}1.95 \\
(1.28,2.98)\end{array}$ & 0.002 \\
\hline & Reference & - & & - & & - & \\
\hline & Long & $\begin{array}{c}0.76 \\
(0.49,1.19)\end{array}$ & 0.231 & $\begin{array}{c}0.91 \\
(0.57,1.48)\end{array}$ & 0.715 & $\begin{array}{c}0.96 \\
(0.65,1.41)\end{array}$ & 0.825 \\
\hline \multirow{3}{*}{ Model $3^{\wedge}$} & Short & $\begin{array}{c}1.50 \\
(0.95,2.36)\end{array}$ & 0.081 & $\begin{array}{c}0.98 \\
(0.67,1.43)\end{array}$ & 0.914 & $\begin{array}{c}2.00 \\
(1.25,3.21)\end{array}$ & 0.004 \\
\hline & Reference & - & & - & & - & \\
\hline & Long & $\begin{array}{c}0.73 \\
(0.47,1.13)\end{array}$ & 0.159 & $\begin{array}{c}0.91 \\
(0.57,1.47)\end{array}$ & 0.706 & $\begin{array}{c}0.96 \\
(0.64,1.43)\end{array}$ & 0.841 \\
\hline \multirow{3}{*}{ Model $4^{\Delta}$} & Short & $\begin{array}{c}1.58 \\
(1.01,2.48)\end{array}$ & 0.047 & $\begin{array}{c}1.02 \\
(0.70,1.49)\end{array}$ & 0.908 & $\begin{array}{c}1.95 \\
(1.22,3.10)\end{array}$ & 0.005 \\
\hline & Reference & - & & - & & - & \\
\hline & Long & $\begin{array}{c}0.74 \\
(0.47,1.15)\end{array}$ & 0.175 & $\begin{array}{c}0.89 \\
(0.56,1.41)\end{array}$ & 0.618 & $\begin{array}{c}1.00 \\
(0.68,1.47)\end{array}$ & 0.995 \\
\hline
\end{tabular}

*Adjusted for age, sex and race-ethnicity

${ }^{\dagger}$ Adjusted for variables in model 1 plus heart rate, obesity, diabetes, hypertension, dyslipidemia, previous cardiovascular disease, congestive heart failure, chronic obstructive pulmonary disease and alcohol intake $\wedge^{\wedge}$ Adjusted for variables in model 2 plus $\beta$-blockers, calcium channel blockers and anti-arrhythmic drugs ${ }^{\Delta}$ Adjusted for variables found significantly associated with the ECG intervals:

- PR interval: age, sex, previous cardiovascular disease, $\beta$-blockers, calcium channel blockers, heart rate

- P duration: age, sex, race-ethnicity, congestive heart failure, alcohol intake, $\beta$-blockers, calcium channel blockers, heart rate

- PR segment: age, sex, alcohol intake, calcium channel blockers, heart rate 
Table 2.1.3 Adjusted survey weighted linear regression between serum cotinine levels and PR interval, $\mathrm{P}$ duration and PR segment

$\mathrm{N}=5,653$

\begin{tabular}{|c|c|c|c|c|c|c|}
\hline & \multicolumn{2}{|c|}{ PR interval (ms) } & \multicolumn{2}{|c|}{$\mathrm{P}$ duration (ms) } & \multicolumn{2}{|c|}{ PR segment (ms) } \\
\hline & $\beta-(95 \% \mathrm{CI})$ & $\begin{array}{c}\mathrm{p}- \\
\text { value }\end{array}$ & $\beta-(95 \% \mathrm{CI})$ & $\begin{array}{c}\mathrm{p}- \\
\text { value }\end{array}$ & $\beta-(95 \% \mathrm{CI})$ & $\begin{array}{c}\mathrm{p}- \\
\text { value }\end{array}$ \\
\hline Model 1* & $\begin{array}{c}-0.832 \\
(-1.549,-0.116)\end{array}$ & 0.0237 & $\begin{array}{c}-0.078 \\
(-0.444,0.288)\end{array}$ & 0.6709 & $\begin{array}{c}-0.755 \\
(-1.355,-0.154)\end{array}$ & 0.0149 \\
\hline Model $2^{\dagger}$ & $\begin{array}{c}-0.593 \\
(-1.320,0.134)\end{array}$ & 0.1074 & $\begin{array}{c}-0.036 \\
(-0.415,0.343)\end{array}$ & 0.8490 & $\begin{array}{c}-0.557 \\
(-1.133,0.019)\end{array}$ & 0.0579 \\
\hline Model $3^{\wedge}$ & $\begin{array}{c}-0.542 \\
(-1.262,0.179)\end{array}$ & 0.1374 & $\begin{array}{c}0.036 \\
(-0.362,0.434)\end{array}$ & 0.8558 & $\begin{array}{c}-0.578 \\
(-1.135,-0.021)\end{array}$ & 0.0424 \\
\hline Model $4^{\Delta}$ & $\begin{array}{c}-0.603 \\
(-1.301,0.094)\end{array}$ & 0.0885 & $\begin{array}{c}-0.008 \\
(-0.391,0.374)\end{array}$ & 0.9648 & $\begin{array}{c}-0.554 \\
(-1.090,-0.018)\end{array}$ & 0.043 \\
\hline
\end{tabular}

*Adjusted for age, sex and race-ethnicity

${ }^{\dagger}$ Adjusted for variables in model 1 plus heart rate, obesity, diabetes, hypertension dyslipidemia, previous cardiovascular disease, congestive heart failure, chronic obstructive pulmonary disease and alcohol intake ${ }^{\wedge}$ Adjusted for variables in model 2 plus $\beta$-blockers, calcium channel blockers and anti-arrhythmic drugs ${ }^{\Delta}$ Adjusted for variables found significantly associated with the ECG intervals:

- PR interval: age, sex, previous cardiovascular disease, $\beta$-blockers, calcium channel blockers, heart rate - P duration: age, sex, race-ethnicity, congestive heart failure, alcohol intake, $\beta$-blockers, calcium channel blockers, heart rate

- PR segment: age, sex, alcohol intake, calcium channel blockers, heart rate 
Table 2.1.4 Association between smoking status and abnormal corrected QT interval and its components

ECG variables groups: Short $\left(<5^{\text {th }}\right.$ percentile $)$, reference $\left(5-95^{\text {th }}\right.$ percentile $)$ and Long $\left(>95^{\text {th }}\right.$ percentile). $\mathrm{N}=5,653$

\begin{tabular}{|c|c|c|c|c|c|c|c|}
\hline & & \multicolumn{2}{|c|}{$\begin{array}{c}\text { Corrected QT } \\
\text { interval (ms) }\end{array}$} & \multicolumn{2}{|c|}{ QRS duration (ms) } & \multicolumn{2}{|c|}{ JT interval (ms) } \\
\hline & & $\begin{array}{c}\text { Odds ratio } \\
(95 \% \mathrm{CI})\end{array}$ & $\begin{array}{c}\mathrm{p}- \\
\text { value }\end{array}$ & $\begin{array}{l}\text { Odds ratio } \\
(95 \% \mathrm{CI})\end{array}$ & $\begin{array}{c}\mathrm{p}- \\
\text { value }\end{array}$ & $\begin{array}{l}\text { Odds ratio } \\
(95 \% \mathrm{CI})\end{array}$ & $\begin{array}{c}\mathrm{p}- \\
\text { value }\end{array}$ \\
\hline \multirow{3}{*}{ Model 1* } & Short & $\begin{array}{c}1.23 \\
(0.87,1.74)\end{array}$ & 0.243 & $\begin{array}{c}1.46 \\
(1.07,1.99)\end{array}$ & 0.017 & $\begin{array}{c}1.41 \\
(0.99,2.02)\end{array}$ & 0.060 \\
\hline & Reference & - & & & & - & \\
\hline & Long & $\begin{array}{c}1.17 \\
(0.71,1.92)\end{array}$ & 0.544 & $\begin{array}{c}1.04 \\
(0.69,1.57)\end{array}$ & 0.842 & $\begin{array}{c}1.50 \\
(0.97,2.32)\end{array}$ & 0.071 \\
\hline \multirow{3}{*}{ Model $2 \uparrow$} & Short & $\begin{array}{c}1.17 \\
(0.84,1.63)\end{array}$ & 0.364 & $\begin{array}{c}1.43 \\
(1.04,1.98)\end{array}$ & $\mathbf{0 . 0 3 0}$ & $\begin{array}{c}0.84 \\
(0.55,1.27)\end{array}$ & 0.409 \\
\hline & Reference & - & & - & & - & \\
\hline & Long & $\begin{array}{c}1.15 \\
(0.68,1.95)\end{array}$ & 0.603 & $\begin{array}{c}1.05 \\
(0.71,1.57)\end{array}$ & 0.799 & $\begin{array}{c}1.70 \\
(1.11,2.61)\end{array}$ & 0.015 \\
\hline \multirow{3}{*}{ Model $3^{\wedge}$} & Short & $\begin{array}{c}1.01 \\
(0.71,1.46)\end{array}$ & 0.946 & $\begin{array}{c}1.60 \\
(1.13,2.26)\end{array}$ & 0.008 & $\begin{array}{c}0.82 \\
(0.52,1.27)\end{array}$ & 0.372 \\
\hline & Reference & - & & - & & - & \\
\hline & Long & $\begin{array}{c}1.29 \\
(0.79,2.11)\end{array}$ & 0.301 & $\begin{array}{c}1.11 \\
(0.71,1.73)\end{array}$ & 0.659 & $\begin{array}{c}1.64 \\
(1.04,2.57)\end{array}$ & 0.032 \\
\hline \multirow{3}{*}{ Model 4 $\Delta$} & Short & $\begin{array}{c}1.05 \\
(0.71,1.54)\end{array}$ & 0.813 & $\begin{array}{c}1.39 \\
(1.02,1.88)\end{array}$ & 0.035 & $\begin{array}{c}0.82 \\
(0.53,1.29)\end{array}$ & 0.392 \\
\hline & Reference & - & & - & & - & \\
\hline & Long & $\begin{array}{c}1.26 \\
(0.79,2.01)\end{array}$ & 0.328 & $\begin{array}{c}1.09 \\
(0.73,1.63)\end{array}$ & 0.682 & $\begin{array}{c}1.81 \\
(1.13,2.89)\end{array}$ & 0.013 \\
\hline
\end{tabular}

*Adjusted for age, sex and race-ethnicity

$\dagger$ Adjusted for variables in model 1 plus heart rate (not for QTc), obesity, diabetes, hypertension, dyslipidemia, previous cardiovascular disease, congestive heart failure, chronic obstructive pulmonary disease and alcohol intake

${ }^{\wedge}$ Adjusted for variables in model 2 plus $\beta$-blockers, calcium channel blockers and anti-arrhythmic drugs ${ }^{\Delta}$ Adjusted for variables found significantly associated with the ECG intervals:

- Corrected QT interval: age, chronic obstructive pulmonary disease, $\beta$-blockers, calcium channel blockers

- QRS duration: sex, obese, chronic obstructive pulmonary disease, heart rate

- Uncorrected JT interval: age, sex, dyslipidemia, alcohol intake, heart rate, anti-arrhythmic drugs 
Table 2.1.5 Adjusted survey weighted linear regression between serum cotinine levels and corrected QT interval, QRS duration and JT interval

$\mathrm{N}=5,653$

\begin{tabular}{|c|c|c|c|c|c|c|}
\hline & \multicolumn{2}{|c|}{ Corrected QT } & \multicolumn{2}{|c|}{ QRS duration } & \multicolumn{2}{|c|}{ JT interval } \\
\hline & $\beta-(95 \% \mathrm{CI})$ & $\begin{array}{c}\mathrm{p}- \\
\text { value }\end{array}$ & $\beta-(95 \% \mathrm{CI})$ & $\begin{array}{c}\mathrm{p}- \\
\text { value }\end{array}$ & $\beta-(95 \% \mathrm{CI})$ & $\begin{array}{c}\mathrm{p}- \\
\text { value }\end{array}$ \\
\hline Model 1* & $\begin{array}{c}-1.448 \\
(-2.375,-0.521)\end{array}$ & 0.0029 & $\begin{array}{c}-0.251 \\
(-0.489,-0.013)\end{array}$ & 0.0391 & $\begin{array}{c}-1.198 \\
(-2.043,-0.354)\end{array}$ & 0.0064 \\
\hline Model $2^{\dagger}$ & $\begin{array}{c}-1.402 \\
(-2.347,-0.456)\end{array}$ & 0.0045 & $\begin{array}{c}-0.184 \\
(-0.414,0.045)\end{array}$ & 0.1126 & $\begin{array}{c}0.130 \\
(-0.327,0.587)\end{array}$ & 0.5701 \\
\hline Model $3^{\wedge}$ & $\begin{array}{c}-1.112 \\
(-2.056,-0.169)\end{array}$ & 0.0218 & $\begin{array}{c}-0.194 \\
(-0.433,0.044)\end{array}$ & 0.1079 & $\begin{array}{c}0.189 \\
(-0.300,0.679)\end{array}$ & 0.4414 \\
\hline Model $4^{\Delta}$ & $\begin{array}{c}-1.345 \\
(-2.269,-0.421)\end{array}$ & 0.0052 & $\begin{array}{c}-0.139 \\
(-0.363,0.086)\end{array}$ & 0.2205 & $\begin{array}{c}0.233 \\
(-0.272,0.738)\end{array}$ & 0.3579 \\
\hline
\end{tabular}

*Adjusted for age, sex and race-ethnicity $\dagger$ Adjusted for variables in model 1 plus heart rate (not for QTc), obesity, diabetes, hypertension dyslipidemia, previous cardiovascular disease, congestive heart failure, chronic obstructive pulmonary disease and alcohol intake

${ }^{\wedge}$ Adjusted for variables in model 2 plus $\beta$-blockers, calcium channel blockers and anti-arrhythmic drugs ${ }^{\Delta}$ Adjusted for variables found significantly associated with the ECG intervals:

- Corrected QT interval: age, chronic obstructive pulmonary disease, $\beta$-blockers, calcium channel blockers

- QRS duration: sex, obese, chronic obstructive pulmonary disease, heart rate

- Uncorrected JT interval: age, sex, dyslipidemia, alcohol intake, heart rate, anti-arrhythmic drugs 


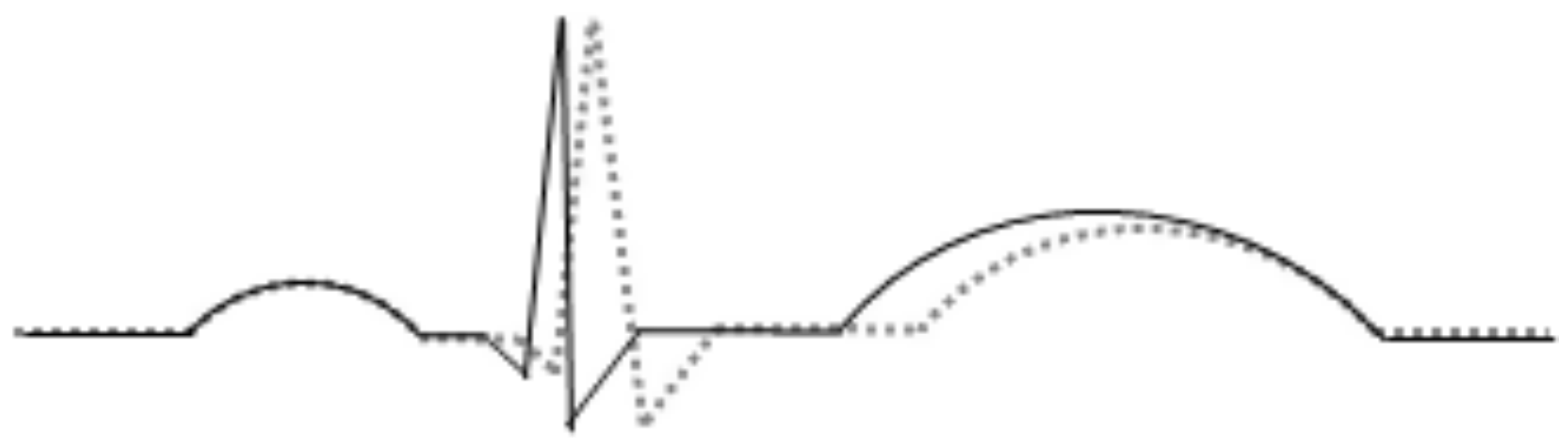

\section{Nonsmoker .................... PR
Smoker \\ ".."PRs}

**nQRS

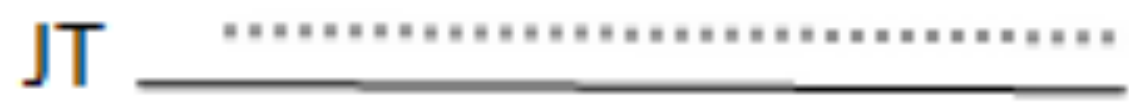

Figure 2.1.1 The effect of smoking status on baseline ECG 
A
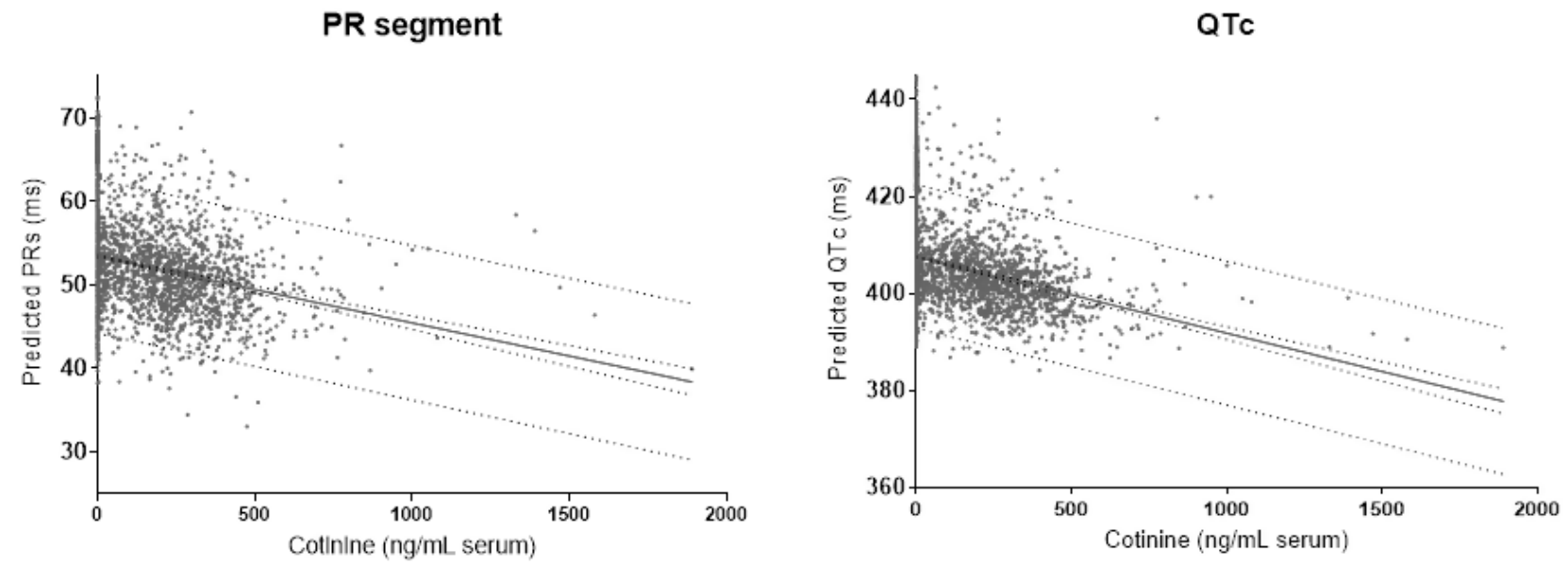

B

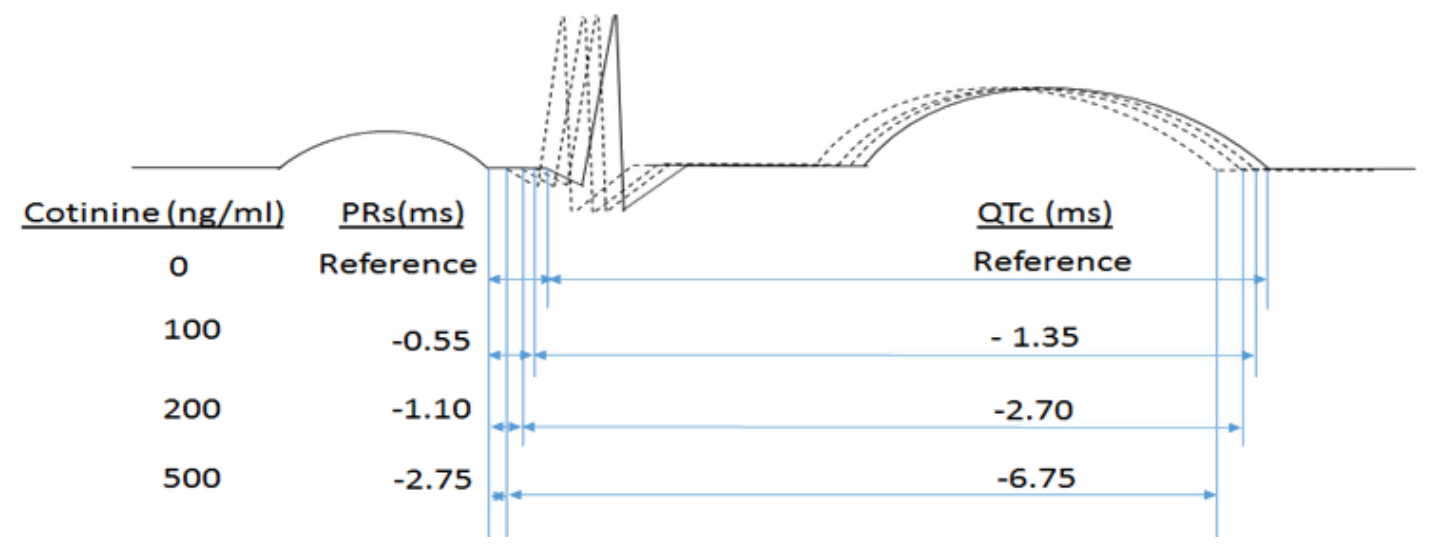

Figure 2.1.2 Panel A shows the distribution of PR segment (ms) and QTc interval (ms) with cotinine levels

$(\mathrm{N}=5,653)$. Panel B depicts the visual representation of corresponding progressive shortening of PR segment and QTc with increasing cotinine levels 


\section{Discussion}

In a large sample representative of the general US population, we found that higher levels of serum cotinine, a measure of tobacco exposure, was associated with extremely shortened PR interval, PR segment, QRS duration and extremely prolonged JT interval. We also found that, although cotinine-confirmed smoking did not associate with extreme changes in QTc, smoking severity as measured by serum cotinine had a significant negative linear relationship with QTc in the overall cohort.

\section{$P R$ interval, $P$ wave and $P R$ segment}

There is a wide variation in the results of prior studies on the effects of tobacco exposure and PR interval and its components: PR segment and P duration [227, 254, 255]. However, studies with greater sample size consistently show smoking associated with shorter PR interval [212, 221, 223, 256-258]. Few studies showed increased P wave duration among smokers [222, 259] while other studies did not show any significant difference, $[225,227]$ or found a trend towards decreasing $\mathrm{P}$ wave duration with smoking [212]. We did not find any significant association between $\mathrm{P}$ wave duration and cotinine levels, suggesting less overt effects of tobacco exposure on atrial activity and atrial conduction. In a smaller cohort we recently revealed an inverse association between cotinine and PR interval, with evidence that this relationship was mediated via increased circulating dopamine[260]. In the present study, our observations spanned a large nationally representative cohort and further corroborate these findings that smoking accelerates atrioventricular conduction. 
These findings are particularly important in light of emerging data on the prognostic value of short PR interval to predict atrial fibrillation [27] and cardiovascular mortality [26, 29, 261]. Therefore, exposure to cigarette smoke (and perhaps nicotine specifically) accelerates atrioventricular conduction (as exhibited by short PR interval) and may possibly be a mechanism for increased risk of stroke and atrial fibrillation.

\section{QTc interval, JT interval and QRS duration}

Similar to the results of studies on PR interval and its components, there are mixed results on the effects of smoking on QTc and QRS duration [212, 228, 229, 262-265]. We were not able to find any study that explored the relationship between JT interval and smoking exposure. Only one study has investigated the effect of smoking, measured specifically by cotinine, on JT interval. Like us, Zhang et al [219] used the NHANES III database, and among 7795 men and women found that in fully adjusted models QTe was not associated with smoking and cotinine levels. At first this appears to conflict with the results of our study, but ancillary analyses (not shown) revealed that this discrepancy stems from their use of quartiles instead of continuous QTc, and their restricting analyses to current smokers only. In addition, we also studied the association of cotinine with the components of QTc interval, and found that higher cotinine levels are associated with abnormally short QRS duration and long JT interval, but without any significant linear relationship with either subcomponents.

The QT interval encompasses the time from the beginning of ventricular depolarization (QRS duration) and ventricular repolarization (JT interval) [170]. Prolonged QRS duration [182] and JT interval [31,32] are well known predictors of mortality in the 
general population, but the clinical implications for short QRS duration are not known. Sympathetic neural stimulation and systemic catecholamine release may underlie smokinginduced acceleration of AV nodal conduction, while also explaining the shortening of QRS (ventricular depolarization)[63, 266, 267] and prolongation of JT (ventricular repolarization) $[194,268,269]$. Nicotine has been found to directly inhibit $\mathrm{IK}_{\mathrm{r}}$ and $\mathrm{I}_{\mathrm{to}}$ repolarizing currents [270]. Notably, nicotine also directly stimulates release of catecholamines from nerve terminals and the adrenal medulla [71, 271], effects that acutely augment $\mathrm{IK}_{\mathrm{s}}$ current and directly cause phosphorylation of its corresponding potassium channel to acutely accelerate repolarization via $\beta$-adrenergic receptors [272]. In addition to increased sympathetic tone and direct effects on ion channels, nicotine may also cause a pro-fibrotic state [273] and endothelial cell injury [274, 275]. Thus, nicotine is a particularly plausible culprit of these effects. Nevertheless, particulate matter and aldehydes within tobacco smoke may be key constituents and irritant reflexes or ischemia may be critical pathways through which smoking disrupts electrophysiologic homeostasis [276]

\section{Limitations}

It has not escaped our attention that a single measurement of serum cotinine may not fully reflect chronic smoking intensity. Moreover, because cotinine only reflects nicotine exposure, and by an imperfect proxy, smoking intensity, we are unable to assess relationships between other cigarette smoke constituents and cardiac electrophysiology. Although we have adjusted for several potential confounders, we recognize the possibility of residual confounding that is similar to other studies with a cross-sectional design. Also, 
though the automated measurement of ECG intervals and segments, are routinely reported and can easily be calculated by the ECG systems, we did not perform manual measurements. Despite these limitations, this is the first study examining the association between serum cotinine, an objective measure of tobacco exposure, with PR and QT interval and their components.

\section{Conclusions}

We found in a large racially diverse sample of the US population, that elevated serum cotinine levels are independently associated with abnormally short PR interval, short PR segment, short QRS duration, and long JT interval. Additionally, increases in cotinine were associated with progressive shortening of QTc across all individuals. Collectively, our findings indicate that exposure to cigarette smoke increases risk for abnormally fast atrioventricular conduction and ventricular depolarization and abnormally long ventricular repolarization. However, smoking induces a progressive prolongation of repolarization when also evaluating individuals within normal QTc ranges. These observations enhance our understanding of the relationship between smoking and cardiac arrhythmias and implicate specific pathophysiological mechanisms by which smoking increases cardiovascular morbidity and mortality, including for stroke and sudden cardiac death. More research is warranted to examine the specificity and selectivity of these effects and to delineate the direct contribution of specific tobacco constituents such as nicotine. 
Effects of cigarette smoking on electrocardiogram mediated via catecholamines

Aim 2 Test the influence of catecholamines in the association between smoking and myocardial conduction.

This study was designed to study the effect of nicotine and cigarette smoke exposure on PR interval and its components in a cohort of patients with intermediate-tohigh CVD risk and to discern the role of sympatho-adrenal activity in these effects on atrioventricular conduction through analyzing urinary metabolites of nicotine and catecholamines (dopamine, norepinephrine, and epinephrine).

\section{Participants from Louisville Healthy Heart Study}

The study was approved by the Institutional Review Board at the University of Louisville. Individuals ( $>18$ years of age) with intermediate to high CVD risk were recruited from the University of Louisville Hospital and affiliated clinic system between October 2009 and March 2011 as described previously [277]. All accessible patients visiting the clinics during this time period were pre-screened through a review of medical records prior to recruitment in order to exclude individuals that did not meet the enrollment criteria. In addition, persons unwilling or unable to provide informed consent or with significant and/or severe comorbidities were excluded. Exclusion criteria included: significant chronic lung, liver, kidney, hematological, or neoplastic disease, chronic neurological or psychiatric illness, chronic infectious disease such as HIV or hepatitis, severe coagulopathies, drug/substance abuse, and chronic cachexia. Pregnant women, prisoners, and other vulnerable populations were also excluded from the study. Patients who met the enrollment criteria and gave written consent were consented and administered 
a questionnaire to provide demographic information and baseline characteristics. Medical records were reviewed for past medical history, vital signs and medication history. To reduce selection bias, all consecutive participants who were eligible for this study were recruited. For our analyses, only those patients with complete urinary biomarkers and ECG with normal sinus rhythm were included (Supplementary Figure 2.2.1).

\section{Electrocardiogram (ECG) measurement protocol}

Standard 12-lead ECGs with 2.5 seconds of each lead and 10 seconds of rhythm strip (lead II) from medical records were used for ECG analyses. The following ECG intervals were measured: $\mathrm{P}$ wave duration (from beginning to end of $\mathrm{P}$ ) and $\mathrm{PR}$ interval from lead II (from beginning of P to beginning of Q) [26], QRS duration from lead V6 (from beginning of Q to end of S) [278], and QT interval from V5 (from beginning of Q to end of T) [279]. The PR segment was calculated as the difference between PR interval and P wave duration. QT was also corrected using the Framingham formula [246] (Appendix A). All intervals were measured with electronic calipers and adjusted to scale by reported automated measures of RR (or heart rate). Two trained analysts (CA and AI) independently and manually measured each ECG interval (except RR) from the first 3 sinus beats. HRV parameters were derived from digital caliper measurements of all RRs in the rhythm strip. When average of any ECG interval for a given patient differed between the two analysts by $>10 \%$, both investigators re-measured that ECG interval independently. If the parameter

remained $>10 \%$ different between analysts, the analysts reviewed the ECG together and reached consensus on the appropriate measure.

\section{Urinary measurements}


A spot urine sample was collected on the day of study enrollment. Urinary cotinine is a well-established metabolite for cigarette smoke exposure [245], and was measured by Ultra performance liquid chromatography - tandem mass spectrometer (UPLC-MS/MS) using D3-cotinine as an internal standard [280]. For UPLC-MS/MS analysis of dopamine, norepinephrine, epinephrine, and their metabolites (metanephrine, normetanephrine, vanillylmandelic acid, 3-methoxytyramine, homovanillic acid), urine samples were thawed on ice, vortexed and diluted 1:50 with $0.2 \%$ formic acid containing isotopic labeled internal standards. $1 \mu \mathrm{L}$ of the mixture was analyzed on an UPLC-MS/MS instrument (ACQUITY UPLC H-Class system and Xevo TQ-S micro triple quadrupole mass spectrometer, all from Waters Inc., MA). Separation was performed on an Acquity UPLC HSS PFP (150 mm × $2.1 \mathrm{~mm}, 1.8 \mu \mathrm{m}$ ) column (Waters Inc., MA) with a binary gradient comprised of $0.2 \%$ formic acid (Solvent A) and methanol (Solvent B). Three multiple reaction monitoring (MRM) transitions were set up for each sample: one for quantification, one for confirmation, and one for labeled internal standard. At least 12 data points were collected for each peak. Analytes were quantified using peak area ratio based on 8 point-standard curves run before and after the urine samples. The concentration values of analytes were normalized to creatinine level which was measured on a COBAS MIRA-plus analyzer (Roche, NJ) with Infinity Creatinine Reagent (Thermo Fisher Scientific, MA). 


\section{Statistical Analysis}

Baseline subject characteristics were summarized by smoking status. Categorical characteristics, frequencies and percentages are reported along with Chi-square test pvalues, which were used to compare distributions across study groups. In addition to visual inspection of histograms, Shapiro-Wilk tests were conducted for continuous characteristics to determine if the characteristics were approximately normally distributed. Mean and standard deviation are reported for continuous characteristics with a normal distribution, whereas median and interquartile range are reported for continuous characteristics with a skewed distribution, and the study groups were compared by the appropriate statistical test based on normality. P-values were derived from Student's $t$-tests for study group comparisons of normally-distributed variables, whereas Mann-Whitney tests were used for variables lacking a normal distribution. The urinary metabolites (cotinine, dopamine, norepinephrine and epinephrine and their daughter metabolites) were log-transformed because their distribution was skewed. We tested the associations between ECG parameters and urinary metabolites by linear regression. ECG parameters were dichotomized by their median levels into high- and low-value groups. Baseline characteristics associated with ECG variables in bivariate analyses (with $p<0.01$ ) were used to build fully adjusted models using linear regression analyses. Smoking status was determined as reported by the participant (active smoker, former smoker or non-smoker) and by urinary cotinine levels of $50 \mathrm{ng} / \mathrm{ml}$ [245]. Finally, mediation was assessed by the bootstrapping technique and macro put forth by Preacher and Hayes [281]. Statistical analyses were performed using Statistical Package for Social Sciences (SPSS) software (version 24, SPSS, Inc, Chicago, IL, USA). 


\section{Results}

\section{Baseline characteristics}

A total of 136 participants were in normal sinus rhythm and had ECGs and urinary metabolites available. The participants were approximately evenly split by gender (male $n=72,53 \%)$, about half of all participants were Caucasians $(n=77,57 \%)$, and mean age was 52 years. Participants had a high prevalence of CVD risk factors; with a majority diagnosed with hypertension $(n=119,87 \%)$ and/or on $\beta$-blockers $(n=98,73 \%)$ (Table 2.2.1). Several of the participants were diagnosed with diabetes $(n=43,32 \%)$ or prior myocardial infarction $(n=62,46 \%)$, and/or were taking calcium channel blockers $(n=32,24 \%)$ or $\beta$-blockers $(\mathrm{n}=98,73 \%)$. The relationship of baseline characteristics with dichotomized PR interval, $\mathrm{P}$ duration, and PR segment are shown in Supplementary Table 2.2.1. Mean age and proportion of females were significantly higher among individuals in the upper stratum for PR interval, whereas mean BMI, or proportion of participants who were female, hypertensive, or taking calcium channel, ACE, or angiotensin receptor inhibitors were higher among those with longer $\mathrm{P}$ duration. Conversely, only the number of participants on diuretics were higher among those with longer PR segment 
Table 2.2.1 Baseline characteristics

\begin{tabular}{|c|c|c|c|c|}
\hline & \multirow{2}{*}{$\begin{array}{l}\text { All patients } \\
\qquad \mathrm{N}=136\end{array}$} & \multicolumn{3}{|c|}{ Current smoker } \\
\hline & & $\begin{array}{c}\text { Yes } \\
(\mathrm{N}=53, \\
39 \%)\end{array}$ & $\begin{array}{c}\text { No } \\
(\mathrm{N}=83, \\
61 \%)\end{array}$ & $\mathrm{p}$ value \\
\hline Age (years)* & 52,10 & 53,10 & 50,9 & 0.05 \\
\hline BMI $(\mathrm{kg} / \mathrm{m} 2)^{*}$ & 33,8 & 34,8 & 31,7 & 0.06 \\
\hline $\mathrm{SBP}(\mathrm{mm} \mathrm{Hg})^{*}$ & 133,23 & 132,22 & 136,24 & 0.15 \\
\hline $\mathrm{DBP}(\mathrm{mm} \mathrm{Hg})^{*}$ & 81,13 & 80,11 & 81,16 & 0.37 \\
\hline Heart rate (beats $/ \mathrm{min})^{*}$ & 73,15 & 74,15 & 73,15 & 0.99 \\
\hline Male gender & $72,53 \%$ & $43,52 \%$ & $30,57 \%$ & 0.58 \\
\hline Caucasian & $77,57 \%$ & $46,55 \%$ & $31,59 \%$ & 0.73 \\
\hline Hypertension & $118,87 \%$ & $74,89 \%$ & $45,85 \%$ & 0.47 \\
\hline Prior MI & $62,46 \%$ & $40,48 \%$ & $23,43 \%$ & 0.58 \\
\hline Diabetes & $43,32 \%$ & $28,34 \%$ & $15,28 \%$ & 0.51 \\
\hline Stroke & $23,17 \%$ & $14,17 \%$ & $10,19 \%$ & 0.77 \\
\hline Arrhythmia & $45,33 \%$ & $28,34 \%$ & $17,32 \%$ & 0.84 \\
\hline B blocker & $98,73 \%$ & $65,78 \%$ & $34,64 \%$ & 0.07 \\
\hline $\mathrm{CCB}$ & $32,24 \%$ & $24,29 \%$ & $8,15 \%$ & 0.06 \\
\hline ACEI or ARB & $89,66 \%$ & $54,65 \%$ & $35,66 \%$ & 0.91 \\
\hline Statin & $84,62 \%$ & $55,66 \%$ & $30,57 \%$ & 0.26 \\
\hline Aspirin & $81,60 \%$ & $49,59 \%$ & $33,62 \%$ & 0.71 \\
\hline Diuretics & $58,43 \%$ & $38,46 \%$ & $20,38 \%$ & 0.35 \\
\hline
\end{tabular}

Abbreviations: BMI - Body mass index, SBP- Systolic Blood Pressure, DBP - Diastolic Blood Pressure, MI - Myocardial Infarction, CCB - Calcium Channel Blocker, ACEI - Angiotensin-Converting Enzyme Inhibitor, ARB - Angiotensin II Receptor Blocker

For each variable counts and column percentage has been reported except for where indicated * where the mean and standard deviation (SD) are reported 
Smoking status and catecholamine levels

Overall smoking prevalence was $61 \%(n=83)$ by self-report and $46 \%$ when defined by urinary cotinine $>50 \mathrm{ng} / \mathrm{ml}$ (Cohen's $\kappa=0.79, \mathrm{p}<0.001$ for agreement between these measures). Three participants self-reported as never smokers had urinary cotinine levels $>50 \mathrm{ng} / \mathrm{ml}$ and two who self-reported as active smokers had urinary cotinine levels of $\leq 50$ $\mathrm{ng} / \mathrm{ml}$ (Supplementary Table 2.2.2). None of the cohort characteristics were significantly different between active smokers and the rest of patients; although trends of higher age, BMI, and use of calcium channel and/or $\beta$-blockers was observed among smokers. All urinary metabolites (cotinine, dopamine, epinephrine and norepinephrine) were higher among current smokers vs never-smokers. None of the catecholamines were different between current smokers and ex-smokers ( $p>0.05)$; however, catecholamines were higher in the high-cotinine group relative to the low-cotinine group (Supplementary Table 2.2.3 A).

Supplementary Table 2.2.4 shows that cotinine was significantly associated with dopamine and norepinephrine, but not with epinephrine. We also explored the possible effect of cotinine on catecholamine metabolism by the association of cotinine and the daughter metabolites and their ratios. Each catecholamine's intermediate metabolite, and none of the final metabolites, had a significant positive association with cotinine. Among the ratio of intermediate/parent metabolites, cotinine associated positively with 3methoxytyramine/Dopamine (an index of catechol-o-methyltransferase activity) and inversely with vanillyl mandelic acid/normetanephrine and vanillyl mandelic acid/metanephrine (both indices of MOA activity). 
Association of cotinine with P- and PR-parameters

Smokers did not differ from non-smokers in proportion of participants within strata of long or short P duration, PR interval, or PR segment. Distribution of participants into high- or low-PR or P-duration parameters also did not differ by cotinine dichotomization (Supplementary Table 2.2.3 B). When analyzed as a continuous variable, cotinine was significantly higher among participants with short PR segment compared to those with long PR segment $(\mathrm{p}=0.03)$. PR interval also showed a similar trend $(\mathrm{p}=0.06)$, whereas cotinine did not differ between participants with long vs. short $\mathrm{P}$ duration $(\mathrm{p}=0.25)$ (Table 2.2.2). Among all participants, cotinine had a significant negative association with PR interval and PR segment (but not with P duration) in linear regressions, even after adjusting for age, gender and heart rate (Table 2.2.3). Figure 2.2.1 shows the scatterplot distributions of PR interval, P duration, and PR segment across log-transformed urinary cotinine levels normalized by creatinine. Cotinine was not significantly associated with other ECG parameters, including QRS duration, QT interval and corrected QT, in unadjusted and adjusted models (Supplementary Table 2.2.5).

\section{Catecholamines and PR interval}

All three catecholamines were significantly elevated among participants with short $\mathrm{P}$ duration relative to those with long P duration (Table 2.2.2). Dopamine was also elevated among those with shorter PR-interval relative to those with longer PR-interval $(\mathrm{p}=0.01)$. Dopamine showed a trend of being higher among patients with short vs. those with long PR-segment. The adjusted linear regression showed all three catecholamines inversely associated with PR interval $(\mathrm{p}<0.05)$, none of the catecholamines associated with $\mathrm{P}$ 
duration, and only dopamine inversely associated with PR segment (Supplementary

Table 2.2.6). 
Table 2.2.2 Comparison of creatinine-normalized urinary biomarkers (median [interquartile range]) among participants dichotomized into high and low atrial and atrioventricular conduction parameters

$\mathrm{N}=136$

\begin{tabular}{|c|c|c|c|c|c|c|c|c|c|c|}
\hline & \multirow{2}{*}{ All patients } & \multicolumn{3}{|c|}{ PR interval } & \multicolumn{3}{|c|}{$\mathrm{P}$ wave duration } & \multicolumn{3}{|c|}{ PR segment } \\
\hline & & $\begin{array}{c}\leq 163.32 \\
\mathrm{~ms}\end{array}$ & $\begin{array}{c}>163.33 \\
\mathrm{~ms}\end{array}$ & $\begin{array}{c}\mathrm{p} \\
\text { value }\end{array}$ & $\begin{array}{c}\leq 104.73 \\
\mathrm{~ms}\end{array}$ & $\begin{array}{c}>104.74 \\
\mathrm{~ms}\end{array}$ & $\begin{array}{c}\mathrm{p} \\
\text { value }\end{array}$ & $\leq 55.20 \mathrm{~ms}$ & $>55.21 \mathrm{~ms}$ & $\begin{array}{c}\mathrm{p} \\
\text { value }\end{array}$ \\
\hline Cotinine (ng/g) & $\begin{array}{c}23.1 \\
{[1.6-794.1]}\end{array}$ & $\begin{array}{c}30.6 \\
{[1.5-948.4]}\end{array}$ & $\begin{array}{c}23.0 \\
{[1.8-647.7]}\end{array}$ & 0.45 & $\begin{array}{c}238.3 \\
{[2.3-102.5]}\end{array}$ & $\begin{array}{c}5.8 \\
{[1.4-627.1]}\end{array}$ & 0.08 & $\begin{array}{c}130.7 \\
{[2.4-1070.1]}\end{array}$ & $\begin{array}{c}9.5 \\
{[1.2-591.3]}\end{array}$ & 0.03 \\
\hline $\begin{array}{l}\text { Dopamine } \\
(\mu \mathrm{g} / \mathrm{g})\end{array}$ & $\begin{array}{c}165.4 \\
{[127.6-} \\
214.1]\end{array}$ & $\begin{array}{c}179.8 \\
{[142.3-} \\
228.5]\end{array}$ & $\begin{array}{c}150.6 \\
{[119.7-} \\
189.5]\end{array}$ & 0.01 & $\begin{array}{c}180.1 \\
{[142.0-} \\
234.7]\end{array}$ & $\begin{array}{c}111.6 \\
{[180.3-} \\
168.8]\end{array}$ & 0.01 & $\begin{array}{c}168.8 \\
{[140.7-} \\
219.8]\end{array}$ & $\begin{array}{c}160.5 \\
{[119.7-} \\
204.9]\end{array}$ & 0.07 \\
\hline $\begin{array}{l}\text { Epinephrine } \\
(\mu \mathrm{g} / \mathrm{g})\end{array}$ & $\begin{array}{c}4.7 \\
{[2.4-7.9]}\end{array}$ & $\begin{array}{c}5.6 \\
{[2.9-8.6]}\end{array}$ & $\begin{array}{c}8.6 \\
{[4.1-7.5]}\end{array}$ & 0.23 & $\begin{array}{c}5.9 \\
{[3.5-9.8]}\end{array}$ & $\begin{array}{c}3.9 \\
{[1.7-6.4]}\end{array}$ & 0.01 & $\begin{array}{c}5.2 \\
{[2.1-8.9]}\end{array}$ & $\begin{array}{c}4.2 \\
{[2.5-7.5]}\end{array}$ & 0.75 \\
\hline $\begin{array}{l}\text { Norepinephrine } \\
(\mu \mathrm{g} / \mathrm{g})\end{array}$ & $\begin{array}{c}37.2 \\
{[26.0-53.3]}\end{array}$ & $\begin{array}{c}36.7 \\
{[26.9-52.6]}\end{array}$ & $\begin{array}{c}38.0 \\
{[22.9-56.4]}\end{array}$ & 0.86 & $\begin{array}{c}41.2 \\
{[30.7-54.5]}\end{array}$ & $\begin{array}{c}31.9 \\
{[22.1-52.3]}\end{array}$ & 0.03 & $\begin{array}{c}36.4 \\
{[28.0-51.7]}\end{array}$ & $\begin{array}{c}37.5 \\
{[22.7-55.6]}\end{array}$ & 0.75 \\
\hline
\end{tabular}


Table 2.2.3 Estimated effects ( $\beta$-coefficients) of an increase in cotinine on PR interval, $\mathrm{P}$ wave, and $\mathrm{P}$ segment, with corresponding $\mathrm{P}$ values, from unadjusted and adjusted linear regressions.

Urinary cotinine was log-transformed. $\mathrm{N}=136$

\begin{tabular}{lcccccc}
\hline & \multicolumn{2}{c}{ PR interval } & \multicolumn{2}{c}{ P duration } & & \multicolumn{2}{c}{ PR segment } \\
& $\beta$ & $\mathrm{p}$ value & $\beta$ & $\mathrm{p}$ value & $\beta$ & $\mathrm{p}$ value \\
\hline Unadjusted Cotinine & -2.38 & $\mathbf{0 . 0 4}$ & 0.33 & 0.73 & -2.70 & $\mathbf{0 . 0 3}$ \\
* Adjusted Cotinine & -2.67 & 0.05 & 0.87 & 0.32 & -2.10 & $\mathbf{0 . 0 4}$ \\
\hline
\end{tabular}

* model adjusted for age, body mass index, and gender (PR interval); BMI, SBP, DBP, gender, hypertension, CCB, and ACEI, and ARB (P duration); or BMI, $\beta$ blocker, and diuretics (PR segment). 
A

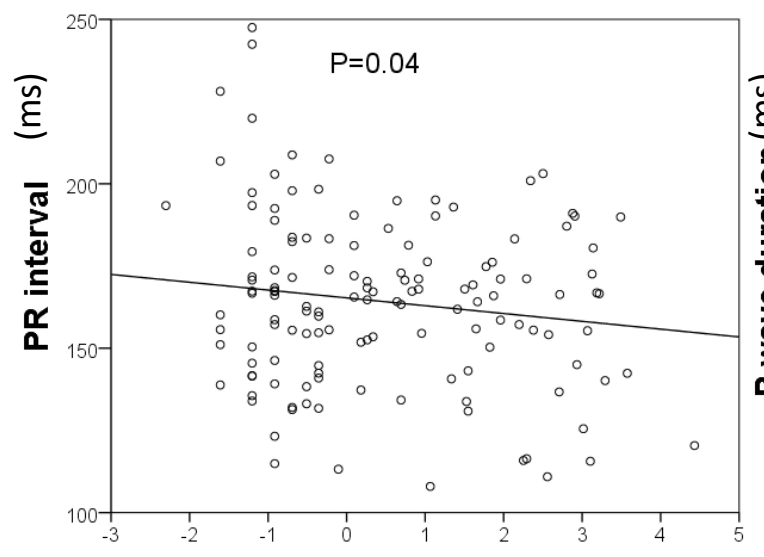

Log transformed cotinine
B

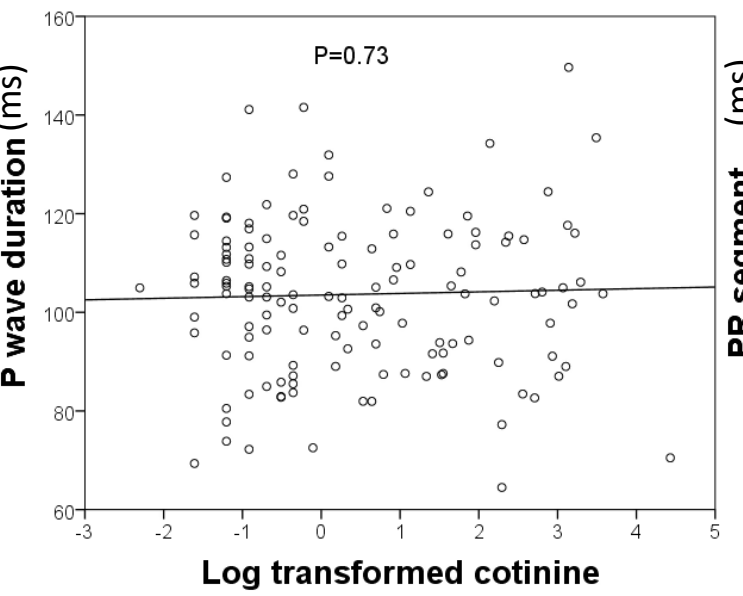

C

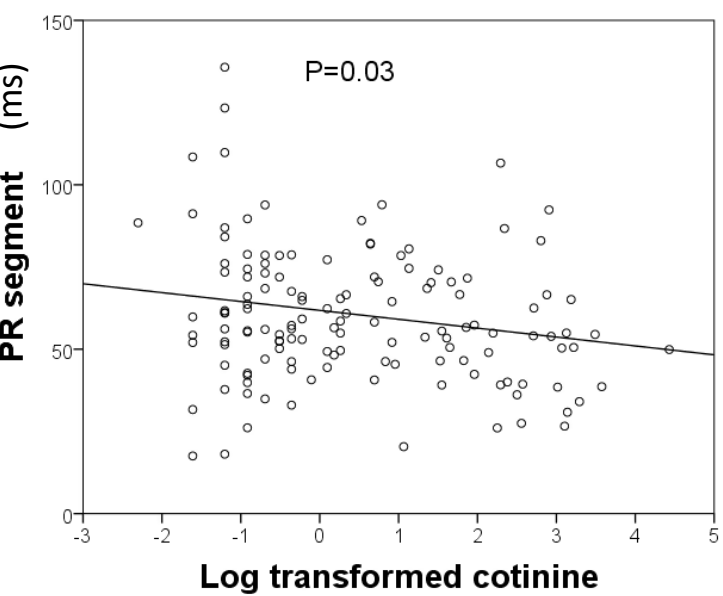

Figure 2.2.1 Scatterplot and linear relationships of (A) PR interval, (B) P wave and (C) PR segment with log transformed urinary cotinine levels

$\mathrm{P}$ values represent unadjusted linear regression. $\mathrm{N}=136$ 


\section{Mediation analyses}

In linear regression analyses cotinine was inversely associated with PR segment and PR interval, and dopamine was the only catecholamine significantly associated with both PR segment and PR interval (inversely in both cases). We therefore conducted mediation analyses to determine whether dopamine mediated the association between cotinine and PR segment and also between cotinine and PR interval. Figure 2 shows the relationship between cotinine and dopamine (path a); the relationship between dopamine and PR interval/segment (path $b$ ); and the total effect of cotinine on PR interval/segment (path $c)$. The total effect (c) is the sum of direct (c') and indirect (ab) effects. The direct effect is the relationship between cotinine and PR interval/segment while controlling for mediators. The indirect effect represents the mediated effect. Mediation analysis showed that dopamine completely mediated the association of cotinine with PR interval (c', $\mathrm{p}=0.46)$ and PR segment $\left(\mathrm{c}^{\prime}, \mathrm{p}=0.09\right)$. Specifically, the indirect effect (path $\left.\mathrm{a} \times \mathrm{b}\right)$ of cotinine on PR interval through dopamine had a point estimate of -1.23 and an upper and lower $95 \% \mathrm{CI}$ of -2.90 and -0.26 ; and the indirect effect (path $\mathrm{a} \times \mathrm{b}$ ) of cotinine on PR segment through dopamine had a point estimate of -0.52 and an upper and lower $95 \% \mathrm{CI}$ of -1.57 and -0.08 (Figure 2.2.2). 


\section{Mediation analyses}

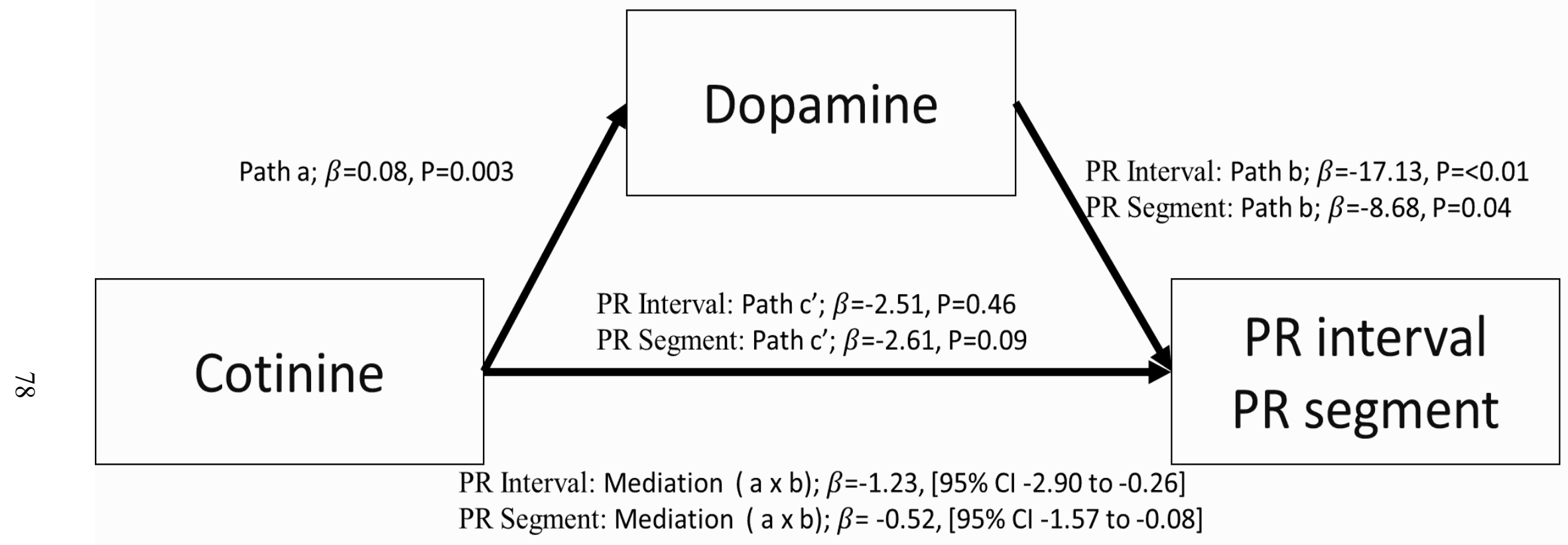

Figure 2.2.2 Mediation analyses of cotinine, dopamine, and PR interval and PR segment

$\mathrm{N}=136$ 


\section{Discussion}

The effects of smoking on atrial and atrioventricular nodal conduction velocity and their underlying mechanisms are currently unknown. To address this gap in knowledge, we measured PR interval, P wave duration, and PR segment from lead II of a 12-lead EKG, urinary catecholamines, and nicotine and its metabolites in a cohort of 136 participants with intermediate-high cardiovascular risk. We report three major findings from this study. First, cotinine had a significant inverse association with PR interval and PR segment, and not with $\mathrm{P}$ wave duration in adjusted linear regression models. Second, urinary dopamine was inversely associated with PR interval and both its components (PR segment and P duration), while epinephrine and norepinephrine did not associate with PR segment. Third, mediation analyses indicate that dopamine may primarily account for the association between cotinine and shortening of both PR interval and segment. Together, these findings suggest smoking may increase cardiovascular morbidity and mortality through nicotineassociated increases in catecholamine release and downstream modulation of atrioventricular conduction.

\section{Nicotine and PR interval and its components}

Previous work on the chronic effects of smoking on PR interval has produced mixed results. Some studies found short PR interval at baseline among chronic smokers vs nonsmokers [212, 221, 257], while others found no difference [254, 282]. Similarly, a few studies showed increased P wave duration among smokers [222, 259], while other studies did not show any significant difference $[225,227]$ or found a trend towards decreasing $\mathrm{P}$ wave duration with smoking [212]. There are several reasons for the wide variation in the 
results of prior studies; 1) small sample size, 2) insufficient consideration for the role of the components of PR interval, and 3) inadequate assessment of nicotine exposure and its potential impact. To the best of our knowledge, to date, no previous study investigated the effects of nicotine exposure on PR interval and its components in humans. This is particularly important in light of recent findings that short PR interval associates with increased risk for atrial fibrillation and cardiovascular mortality [26, 29, 261, 283]. Beyond overall cardiovascular mortality, smoking also associates with increased risk of atrial fibrillation through unknown mechanisms [284]. Thus, by demonstrating that exposure to cigarette smoke (and perhaps nicotine specifically) accelerates atrioventricular conduction, our findings provide further insight into how smoking may confer cardiovascular risk, including risk for atrial fibrillation.

In the present study, cotinine inversely associated with PR interval and PR segment, indicating that increased cigarette smoke exposure accelerates atrioventricular conduction. PR interval is mainly influenced by the sum of atrial activity and atrioventricular nodal conduction [170]. Atrial pathology usually results in prolonging (rather than shortening) of PR interval, whereas shortening of PR interval is likely a result of accelerated atrioventricular nodal conduction. Hence, it is plausible from our results that chronic nicotine exposure expedites atrioventricular nodal conduction. Moreover, because P wave duration was not associated with cotinine, our findings suggest nicotine has limited effects on atrial conduction. Using our linear regression coefficient, we found that each $100 \mathrm{ng} / \mathrm{ml}$ cotinine is associated with a 12.3-ms decrease in PR interval and 9.7-ms decrease in PR segment. 


\section{Cotinine and catecholamines}

Cigarette smoking and nicotine result in increased central and peripheral sympathoadrenal activation. The activation of nicotinic acetylcholine receptors in the adrenal medulla leads to increased catecholamine levels [285]. We found urinary dopamine and norepinephrine (but not epinephrine) were significantly higher among smokers and those with higher cotinine, suggesting smokers have chronically increased sympathetic neuronal activity (norepinephrine), but similar adrenal medullary hormone secretion (epinephrine). Interestingly, we also found that the intermediate metabolites of all three catecholamines significantly associated with cotinine, but not with the final product, and there was a negative association between cotinine and the ratio of the final and intermediate metabolite. This suggests cigarette smoking is associated with increased synthesis of dopamine and norepinephrine and at the same time decreases the catabolism of the intermediate metabolites of all three catecholamines. This corroborates the findings of several other studies that showed cigarette smoking inhibits the activity of MOA [286, 287]; an enzyme primarily responsible for catabolism of the intermediate metabolites of norepinephrine and epinephrine to their final metabolites.

There are several mechanisms through which nicotine can possibly increase atrioventricular nodal conduction velocity. Nicotine stimulates sympathetic neurotransmission via activation of nicotinic acetylcholine receptors localized on peripheral postganglionic sympathetic nerve endings and the adrenal medulla which in turn cause catecholamine release [91, 285]. Catecholamines mediate positive chronotropic, inotropic, dromotropic, and bathmotropic effects (i.e., increased rate, force conductivity and excitability) [288]. Furthermore, evidence from animal models and ex-vivo studies 
suggests nicotine may also directly cause endothelial cell injury [275], a pro-fibrotic state [273], and inhibition of cardiac A type potassium channels [85].

The mediation analysis is a novel component to our study that reveals, among catecholamines, dopamine fully mediates the effect of cotinine and shortening PR interval/segment. The positive dromotropic effects of dopamine accelerate atrioventricular nodal conduction $[289,290]$, thereby expected to shorten the PR interval/segment without affecting $\mathrm{P}$ wave duration, as seen in our study.

Cotinine was even associated with altered metabolism of the three catecholamines (ratio of metabolite/parent). The positive association of cotinine with 3methoxytyramine/Dopamine ratio suggests smoking increases dopamine synthesis and/or systemic secretion and, in compensation, also increases catechol-O-methyltransferase (COMT) activity. Also, cotinine's inverse association with Vanillylmandelic acid/Normetanephrine and Vanillylmandelic acid/Metanephrine suggest that smoking decreases MAO activity, consistent with observations of decreased MAO-B in the amygdalae of smokers and the antidepressant effects of nicotine [291, 292]

\section{Catecholamines and PR interval}

Interestingly, in our study only dopamine and not epinephrine and norepinephrine were associated with PR interval and PR segment. As all catecholamines are known to exert a dromotropic effect, plausible reasons for our finding include: 1) short and long term stress (smoking) disproportionately affect circulating dopamine relative to norepinephrine and epinephrine [293], perhaps secondary to nicotine-mediated declines in dopamine uptake [294]; 2) cigarette smoking and/or nicotine cause an immediate surge in dopamine 
from neuronal [9], that may cross the blood brain barrier to increase circulating dopamine; 3) as the precursor of both epinephrine and norepinephrine, dopamine may have less temporal variability, making it a more stable marker of chronic SNS activation; and 4) at low pathophysiological stress levels, dopamine may have higher affinity than epinephrine and norepinephrine for $\beta-1$ - and $\beta$-2- adrenergic receptors, which modulate dromotropy in the atrioventricular node [295] .

\section{Other ECG parameters}

We did not observe any association of smoking status or cotinine with QRS, QT or cQT and. Previous studies in this area have yielded mixed results, including QT prolongation [229, 262, 263], QT shortening [228], or no relationship [219] with smoking.

\section{Limitations}

While this is the first known investigation of the influence of catecholamines in nicotine-mediated alterations in cardiac conduction, the sample size is limited and participants were drawn at random from the outpatient clinic setting with intermediate to high cardiovascular risk. Thus, we might have missed significant associations between cotinine and other parameters (e.g., P-wave duration) due to insufficient statistical power. Additionally, ECGs were obtained retrospectively from medical records and not on the day of enrollment. The median and interquartile range of number of days from study enrollment (urine sample collection) and ECG was 79 [20 - 320] days. We did not collect data on time of last cigarette/nicotine exposure and states that may affect catecholamines (stress, noise, discomfort, body position, consumption of food, caffeinated beverages and drugs). 
Nevertheless, we used urinary analytes (cotinine and catecholamines) that are established markers for chronic nicotine and sympatho-adrenal activation and are unlikely to exhibit large acute variation in the outpatient clinic setting from which this cohort was derived. We did not collect 24-hour urine to account for diurnal and intra-individual variation in catecholamines [296]; however, summative analysis of 24-h catecholamine production may mask elevations due to dilution. Importantly, a HRV parameter (RMSSD: square root of the mean of squared differences of successive NN intervals), when dichotomized, tended to inversely associate with urinary norepinephrine and dopamine $(r=-0.13, p=0.10$ for both), suggesting concordance between measures of sympathetic activity in both ECG and subsequent urine samples. Although most participants were on $\beta$-blockers, prevalence did not significantly differ by cotinine strata, and PR and P wave durations did not differ by $\beta$ blocker use. This latter point accords with observations that $\beta$-blockers do not alter resting PR interval whereas they partially attenuate PR and RR interval shortening during exerciseinduced sympatho-excitation [297, 298]. Additionally, we were unable to assess associations between other cigarette components or smoking habits (e.g., frequency) and cardiac electrophysiology. Other constituents within tobacco smoke (e.g., particulate matter and aldehydes) have been shown to alter autonomic balance and may thus plausibly alter catecholamine synthesis, secretion, and metabolism. Finally, smoking and nicotine can affect myocardial conduction velocity through induction of cardiac remodeling, oxidative stress, and/or ion channel dysfunction, which were not assessed in this study and may occur independent of sympathetic activation. Nevertheless, sympathetic activation can induce all three of these pathogenic processes [299] and may thereby indirectly mediate atrioventricular conduction defects. 


\section{Conclusions}

Collectively, our findings suggest that exposure to cigarette smoke accelerates atrioventricular conduction and that dopamine mediates these effects. More research is warranted to examine the specificity and selectivity of these effects and to delineate the direct contribution of nicotine. These observations identify a pathway by which smoking may increase risk for cardiovascular morbidity and mortality. 


\section{CHAPTER III}

\section{ACUTE EFFECTS OF NICOTINE AND CIGARETTE SMOKING ON ELECTROCARDIOGRAM}

Aim 3 Acute effects of cigarette smoking and nicotine with and without $\beta$-blocker on electrocardiogram.

Cigarette smoking has acute deleterious effects on the cardiovascular system, resulting in increased incidence of coronary artery disease, atrial and ventricular arrhythmias and sudden cardiac death among smokers [300, 301]. Despite, a strong doseresponse relationship between cigarette smoking and CVD, it remains unclear which tobacco smoke constituents and biological pathways mediate this increased risk. The main addictive component in cigarette smoke, nicotine, has been implicated as possibly the major mediator of acute cigarette smoke induced ANS dysregulation of the heart via increased sympathetic nerve activity and multiple downstream mechanisms [133]. Imbalance within the ANS is increasingly recognized as a major culprit of CVD. Indeed, both acute and chronic activation of the sympatho-adrenal system, promotes cardiovascular dysfunction and disease, including arrhythmia, hypertension, heart failure, myocardial infarction, and ischemic stroke $[302,303]$. The rising popularity of ENDS, and the enhanced nicotine dose of some ENDS devices relative to conventional smoking [304], have created an imperative to determine the cardiovascular effects of nicotine and the role of autonomic imbalance within them. 
The ECG is a widely available, inexpensive, non-invasive, routine method of measuring electrical activity of the heart, and can be used to screen high risk populations and identify ad predict CVD. Several ECG parameters have also been used clinically to assess cardiovascular autonomic dysfunction as a diagnostic and prognostic factor. There is now abundant evidence that acute ANS imbalance has profound effects on electrophysiology [305, 306]. Further, acute variations in hemodynamics [307] and conventional ECG parameters, including dispersion of ECG intervals and amplitudes, may result from acute ANS imbalance and, when frequent, carry worse long term prognosis [308-310]. Additional parameters have recently been added to the repertoire of ECG indices that reliably predict cardiovascular morbidity and mortality (i.e., $\mathrm{P}$ wave amplitude, PR interval, and JT interval) [26, 30, 283, 311-313].

Despite known cigarette induced acute ECG changes and powerfully predictive value of ECG changes for future CVD events, no prior study has systematically 1) evaluated the acute temporality of the acute electrocardiographic effects of smoking, and 2) compared these with nicotine alone, and 3) explored the potential underlying mechanisms. To the best of our knowledge, this is the first report on the immediate effects of cigarette smoking and nicotine on ECG morphologic endpoints. We therefore conducted an open label 2 x 2 factorial experimental trial to study the acute effects of smoking and nicotine, with and without $\beta$-blocker. 


\section{Participants and setting of the experimental trial}

Study design

Participants were recruited via online and flyer advertisements in the public areas city of Huntington, WV. The study enrolled healthy adult male and female smokers, aged 18-65 years, who had smoked at least 10 cigarettes/day for at least one year prior to the trial. Eligible participants had to weigh at least $55 \mathrm{~kg}$ and have a body mass index (BMI) within the range $17.5-30.0 \mathrm{~kg} / \mathrm{m} 2$. Females who were pregnant or breast feeding, vulnerable population (such as patients with mental illness, prisoners etc), and participants with any medical illness requiring routine medications were excluded from the trial. Participants were screened over the phone call before entering the trial. The study design is shown in Supplementary Figure 3.1 A

\section{Participants}

The study was approved by the Institutional Review Board at Marshall University. Each participant signed an informed consent before inclusion in the study. Twenty healthy smokers (65\% men; $37 \pm 13$ years of age; body mass index, $28 \pm 5 \mathrm{~kg} / \mathrm{m} 2)$ and ten healthy non-smokers smokers ( $70 \%$ men; $28 \pm 5$ years of age; body mass index, $26 \pm 3 \mathrm{~kg} / \mathrm{m} 2)$ were included in the study. All of the smokers were regular habitual cigarette smokers $(22 \pm 8$ cigarettes per day for $21 \pm 12$ years). The baseline characteristics along with details of smoking history and cigarette are provided in Supplementary Table 3.1. Except for one smoker (who used rescue inhaler occasionally for well controlled mild asthma), no other participant had any known medical illness nor routine medication use (including birth control pills). All subjects were at least high school graduates. 
Study visits and exposures

All participants had abstained from food, coffee, and tobacco overnight before each study visit. Each smoker completed two-day visits, where on their first day they smoked single preferred brand of Combustible Cigarette (Cig) in their usual manner, and second day they were administered $4 \mathrm{mg}$ of nicotine from Nicotine mouth Spray device $(\mathrm{NicS})$ designed to mimic the acute nicotine delivery of cigarettes. Ten of the twenty smokers were randomly invited for two additional visits, where the exposures remained the same (i.e. preferred single combustible cigarette smoke on day 3 and $4 \mathrm{mg}$ nicotine spray on day 4 ), but were pre-treated with $80 \mathrm{mg}$ oral propranolol (non-selective $\beta$-blocker) for two hours prior to the start of the study (BB-Cig and BB-NicS). The oral propranolol was administered by physician, and participant's vitals were closely monitored during the day 3 and 4 . Ten of the twenty smokers also completed a fifth visit to serve as controls, where they simulated smoking by inhaling through their unlit cigarette ("Sham"), for the similar duration of smoking period on Cig.

The nicotine mouth spray device (Nicorette oromucosal nicotine spray, $1 \mathrm{mg} /$ spray, McNeil AB) consists of a plastic bottle with $13.2 \mathrm{ml}$ of a clear to slightly opalescent liquid with a mint flavor. After priming, one depression of the spray nozzle delivers a metered dose of $1 \mathrm{mg}$ of nicotine in $0.073 \mathrm{ml}$ of a $10 \%$ ethanol-in-water solution. The spray liquid also contains very small amounts of levomenthol $(0.7 \mathrm{mg} / \mathrm{spray}$ dose $)$ and other flavorings (less than $0.3 \mathrm{mg} /$ spray dose) [314]. The $4 \mathrm{mg}$ of nicotine was administered by study personnel by pressing the nozzle four times in rapid succession, directed straight into the participant's mouth. Participants were advised to avoid respiring during, and swallowing 
immediately after, administration of the spray. The dose-concentration curve from the oral spray (Cmax $9.1 \mathrm{ng} / \mathrm{ml}$ and Tmax, 10 minutes, $\mathrm{T} 1 / 22.6 \mathrm{hr}$ ) [314], mimics that of smoking cigarette with about $1 \mathrm{mg}$ of nicotine content $(\mathrm{Cmax} 11.9 \mathrm{ng} / \mathrm{ml}$ and Tmax, 8 minutes, $\mathrm{T}$ $1 / 22.5 \mathrm{hr})[315]$.

\section{Procedures}

All study visits were separated by washout periods of at least 24 hours, and were started between $8 \mathrm{AM}$ and 10:00 AM. After arrival to the research clinic, and obtaining informed consent, each participant completed a detailed health and tobacco use questionnaire. Height and weight were measured. There was a rest of 5 minutes before each blood pressure measurement and ECG recording session. Pre and post exposure blood pressure were measured in sitting position using a validated automated monitor, in accordance with the guidelines [316]. Pre and post exposure serum and plasma were also collected, and stored at $-20^{\circ} \mathrm{C}$ until analysis (except for on Sham smoking day). A standard continuous 12 lead ECG was recorded (Cardio Card, Nasiff Associates, Central Square, NY), using as per guidelines [317], for 5 mins indoor while supine (pre-exposure), 20 mins outside while seated (during exposure) and 5 mins indoors while supine (post-exposure). The exposures were always performed while seated in the same spot; which was outside in an open public area, (200 feet away from the research building); which was not a designated public smoking place and away from main road traffic area. Supplementary Figure 3.1 B and Appendix B show the schematic timeline of the study day protocol. 


\section{ECG and HRV analyses}

Data from Lead II was extracted and each beat was analyzed using the commercially available software LabChart/ECG and HRV Analysis Add-On (version 8.0; ADInstruments, Colorado Springs, CO, USA) (Appendix C). The artifacts were identified and removed from the analysis using the ECG Beat Classifier; which measures the Activity (sample-to-sample voltage differences), isoelectric noise, form factor (shape and time course of QRS complex) and RR interval. Supplementary Figure 3.2 depicts a typical ECG waveform, with the intervals and amplitudes that were measured automatically by the software. The data was further cleaned by excluding variables with $>20 \%$ difference from the median of 6 surrounding beats. To decrease variance, the ECG waveforms were averaged over three-minute time intervals during the 20 minutes exposure, and were averaged over 5 minutes during the pre- and post-exposure. For HRV, Time and frequency domain analysis were performed, at 5 min intervals and the entire 20 minutes during exposure, to record root mean square of successive differences (RMSSD) and standard deviation of normal beat intervals (SDNN) and LF/HF (ratio of LF to HF power), using the spectral settings as per the international guidelines [166].

\section{Plasma measurements}

The collected blood samples were immediately centrifuged and the plasma fraction were frozen until the end of study for batch analysis. The plasma Nicotine, Cotinine and Trans-3'-Hydroxy Cotinine in plasma samples were measured by UPLC-MS/MS using a Xevo TQ-S micro quadrupole mass spectrometer with an ESI ionization source, interfaced 
with Waters Acquity Class-H UPLC equipped with a quaternary pump system (Waters, MA). Details about the procedure is given in Appendix $\mathbf{D}$.

\section{Statistical analyses}

Each participant's absolute change $(\Delta)$ for ECG waveforms (intervals and amplitudes), and HRV variables were calculated from the pre-exposure (baseline). The QTe was calculated by using Bazett formula (QT Interval / $\sqrt{ }$ (RR interval). The Area Under the Curve (AUC) for the ECG parameter changes from baseline during exposure (20 minutes) were calculated using the trapezoidal rule [318] for each participant and as average for all participants per study visit. Time series physiologic data are presented as change from baseline to allow consistent comparison to reported effects of cigarette, nicotine, with and without propranolol. We analyzed time-series deltas (each participant's change during exposure from their own value at baseline) with linear mixed effects models (PROC MIXED) for different exposure effects using SAS 9.3 (SAS Systems; Cary, NC). A trendline function was also derived for ECG parameter that had significant change from Sham, and utilized LINEST function without 'forced-intercept' [319], along with six different regression types, to find the best fit curve, while maximizing the R-square and minimizing the variance to avoid under- and over-fitting. Spearman correlation was used to assess the relationship between the $\Delta$ in nicotine and its metabolites with the change in ECG parameters and Blood Pressure. The potential mediation of effects on ECG morphology by acute changes in autonomic balance (measured by RMSSD) was assessed by the bootstrapping technique and a macro put forth by Preacher and Hayes [281]. 
A paired t-test was used to calculate the difference in blood pressure pre and post exposure. All statistics with $\mathrm{P}<0.05$ were considered significant, and were performed in SAS 9.3 and Statistical Package for Social Sciences (SPSS) software (version 24, SPSS, Inc, Chicago, IL, USA). We also created a typical one-minute ECG waveforms, from pre and during exposure, to represent the ECG changes in a Figure, between different exposures, using ecgAuto, v3.3 (Emka Technologies, Paris, France).

\section{$\underline{\text { Results }}$}

On Cig, NicS and Sham days, the baseline (pre-exposure) ECG parameters, except for QTc and JTc, were similar. Pre-treatment with propranolol, decreased the Heart Rate, and increased the RMSSD and SDNN and the ST height. There was no significant difference in any baseline ECG parameter between BB-Cig and BB-NicS days (Supplementary Figure 3.3).

The AUC for each exposure and study visit along with Sham is shown in Figure 3.1, while the raw values are shown in Supplementary Figure 3.4.

Heart Rate and HRV: Cig and NicS both immediately increased heart rate, which peaked at minutes 3 to $6(+20$ beats/minute and +14 beats/minute, respectively). After both exposures, the heart rate remained elevated up-to 20 minutes and returned to baseline post exposure. However, the AUC of heart rate for Cig was higher than that for $\operatorname{NicS}(\mathrm{p}<0.05)$. Pre-treatment with propranolol had a significant impact on HR, with much lower AUC observed with BB-Cig vs Cig alone; while there was a non-significant decrease in AUC for BB-NicS vs NicS day. Cig immediately decreased RMSSD (within first 5 minutes and lasting for 10 minutes), while NicS had delayed albeit significant and similar decrease in 
HRV measurements at 10 minutes. The overall significant decrease (vs Sham) in AUC for RMSSD was similar between Cig and NicS ( $\mathrm{P}>0.05)$. Despite RMSSD increased after treatment with propranolol on both days, cigarette smoke decreased the RMSSD ( $-50 \mathrm{~ms}$ ) vs Cig day $(-20 \mathrm{~ms})(\mathrm{p}<0.05)$; while the AUC for NicS and BB-NicS was similar. Similar patterns were observed with SDNN and LF/HF.

Atrial indices: The smoking and NicS related changes from baseline in $\mathrm{P}$ duration were not different from those of Sham. However, cigarette use induced immediate and significant shortening of PR interval (-14 ms at $9^{\text {th }}$ minute) and PR segment $\left(-10 \mathrm{~ms}\right.$ at $9^{\text {th }}$ minute) $(\mathrm{P}<0.05$ vs Sham), and this effect was abolished with pre-treatment with propranolol ( $\mathrm{P}>0.05$ vs. Sham), while NicS had no significant impact on PR segment. Both, Cig and NicS significantly and similarly increased the P amplitude at 3-6 minutes of exposure compared to Sham ( $+21 \mu \mathrm{V}$ and $+28 \mu \mathrm{V}$, respectively), and interestingly this effect remained unaltered by propranolol. The AUC of P amplitude for Cig was small because, this effect reversed directionality to become negative (though not significantly) at 9-12 minutes of exposure (i.e. the above zero baseline AUC was similar between Cig and $\mathrm{NicS})$.

Ventricular indices: Cig caused greater shortening of QTc ( $-11 \mathrm{~ms})$ relative to NicS ( $-4 \mathrm{~ms})$, and both Cig and Nic significantly shortened QTc relative to Sham $(\mathrm{P}<0.05$ vs. Sham). On BB days, this effect was abolished and the AUC for BB-Cig and BB-NicS were similar to Sham, and higher than that of Cig and NicS, respectively. Cig immediately shortened QTc in the first 3 min, with a subsequent plateau effect for the remainder of the exposure and returning to baseline in the post-exposure period. Cig and NicS similarly deepened Q wave amplitude ( $-59 \mu \mathrm{V}$ and $-72 \mu \mathrm{V}$, respectively, $\mathrm{P}<0.05$ vs. BL or Sham), 
with propranolol blocking that effect. There was no significant effect of Cig or NicS on QRS and Tp-Te durations. The ST height gradually decreased after Cig and significantly differed from Sham minutes 3-6 $(-27 \mu \mathrm{V})$, with maximum effect at 9-12 minutes $(-41 \mu \mathrm{V})$ and slowly subsiding thereafter (remaining significantly decreased at minute 18-21) to baseline levels in the post exposure period. NicS had no such effect, and propranolol abolished the Cig-induced ST depression.

The typical 1-minute average ECG morphology during six exposures are shown in Figure 3.2. Figure 3.3 shows the best fitting equation for the trendline of the changes in Heart Rate, P amplitude, QTc from Cig and NicS and PR segment and ST height from Cig. Supplementary Figure 3.4 show the raw and relative of the important ECG parameters on different days among smokes.

Nicotine and its metabolites: The raw and relative changes in nicotine, cotinine and $3 \mathrm{OH}$ were similar among different visit days of smokers; and higher compared to nonsmokers (Supplementary Figure 3.5; $\mathrm{p}<0.05$ for each biomarker). There was significant correlation between change in nicotine levels with that of heart rate, ST-height, PR interval, PR segment, Q amplitude, SDNN and RMSSD among smokers (Cig and NicS days) and non-smokers (Table 3.1). 
Table 3.1 Correlation between Area under the Curve of ECG parameters with change in Nicotine, Cotinine and 3-hyroycotinine (3-OH) among smokers on Cig-Day and NicSDay and Non-smokers

\begin{tabular}{lccc}
\hline & $\Delta$ Nicotine & Pre-Cotinine & Pre-3HC \\
\hline Heart Rate & $\mathbf{0 . 4 4 3 * *}$ & 0.165 & 0.099 \\
ST height & $\mathbf{- 0 . 2 9 9 *}$ & -0.246 & -0.155 \\
PR interval & $\mathbf{- 0 . 3 0 3 *}$ & -0.015 & 0.077 \\
PR segment & $\mathbf{- 0 . 3 1 8 *}$ & 0.081 & 0.135 \\
P duration & 0.015 & -0.021 & -0.062 \\
P amplitude & 0.123 & $\mathbf{0 . 3 0 4 *}$ & 0.219 \\
QTc interval & -0.140 & -0.180 & -0.084 \\
QRS duration & 0.191 & 0.128 & -0.039 \\
Q amplitude & $\mathbf{- 0 . 3 6 4 * *}$ & -0.149 & 0.004 \\
SDNN & $\mathbf{- 0 . 3 4 4 *}$ & -0.126 & -0.157 \\
RMSSD & $\mathbf{- 0 . 4 4 6 * *}$ & -0.147 & -0.126 \\
Systolic Blood Pressure & $\mathbf{0 . 4 3 8 * *}$ & 0.162 & 0.148 \\
Diastolic Blood Pressure & $\mathbf{0 . 3 7 4 * *}$ & 0.003 & -0.061 \\
\hline
\end{tabular}

** Correlation is significant at the 0.01 level (2-tailed).

* Correlation is significant at the 0.05 level (2-tailed).

ANS and other ECG parameters: To test for statistical mediation of the electrophysiologic effects of Cig and NicS exposures by ANS imbalance, we also evaluated the correlation of RMSSD with the other ECG parameters (Supplementary Table 3.2) where only Heart Rate and ST-height correlated with the nicotine levels among smokers on Cig-day and non-smokers. Mediation analysis showed that RMSSD completely mediated the association of nicotine with ST-height; specifically, the indirect effect (path 
$\mathrm{a} \times \mathrm{b}$ ) of $\Delta$ nicotine on ST-height through RMSSD had a point effect estimate ( $\beta$ ) of -26.57 and an upper and lower $95 \% \mathrm{CI}$ of -82.80 and -0.90 . While change in nicotine directly affected Heart Rate ( $c^{\prime}$ path; $p=0.036$ ), and this effect was independent of changes in RMSSD (Figure 3.4).

There was no effect of baseline cotinine on the relationship between changes in nicotine and that of ECG parameters (Heart Rate, PR segment, ST height and root mean squared of successive differences (RMSSD)), among smokers on Cig-Day and NicS-Day and Non-smokers (Supplementary Figure 3.6).

Blood pressure: Both Cig and NicS similarly and significantly increased postexposure systolic and diastolic blood pressures relative to Sham and non-smokers (Supplementary Figure 3.7). At pre-exposure, blood pressures were similar between Cig, NicS and Sham. There was no significant change in blood pressure on the BB days post Cig and post NicS. The change in nicotine levels also significantly correlated with the changes in blood pressure (Table 3.1), which were not observed on BB days ( $\mathrm{p}>0.05$ ). 

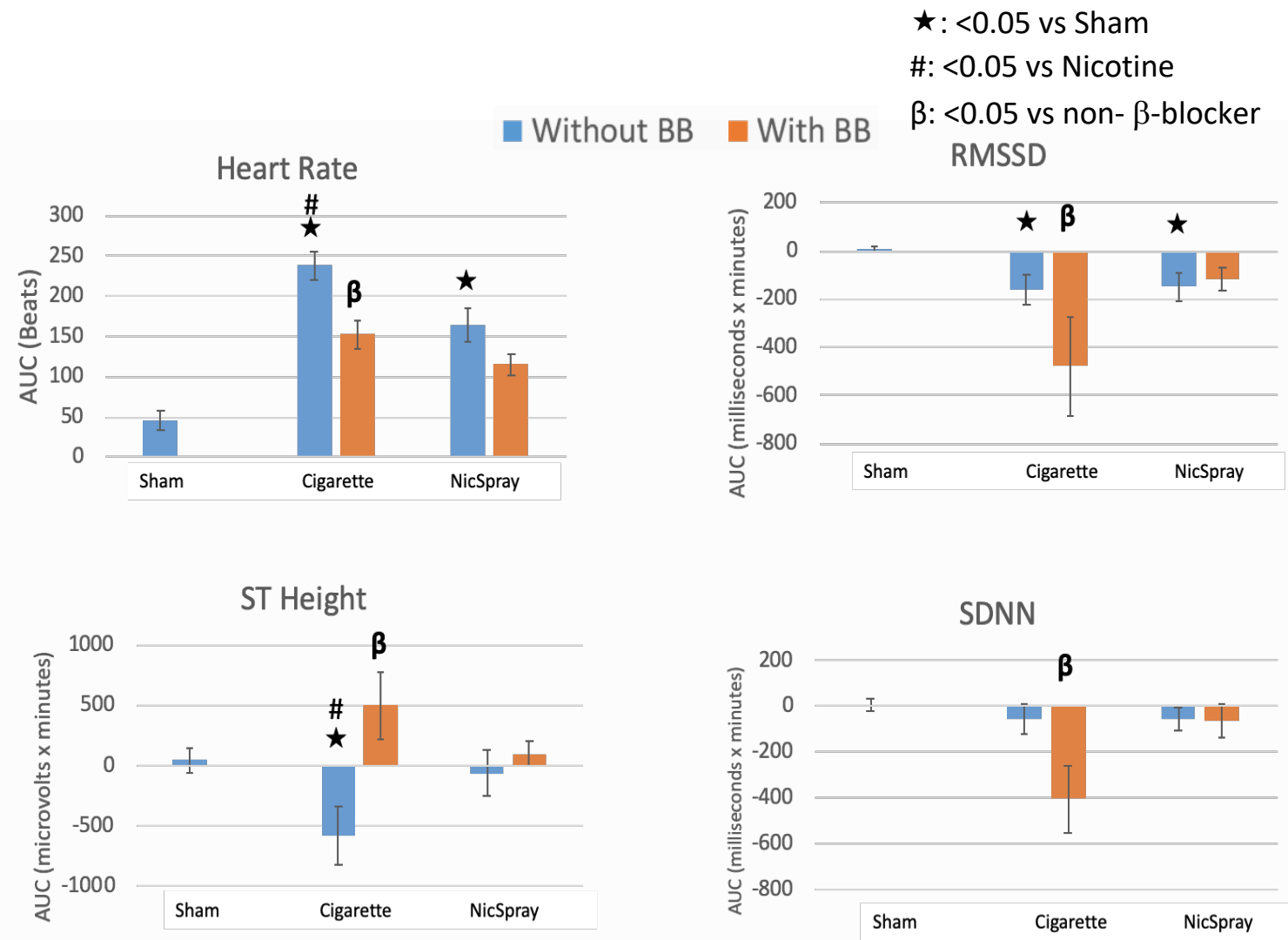

PR interval
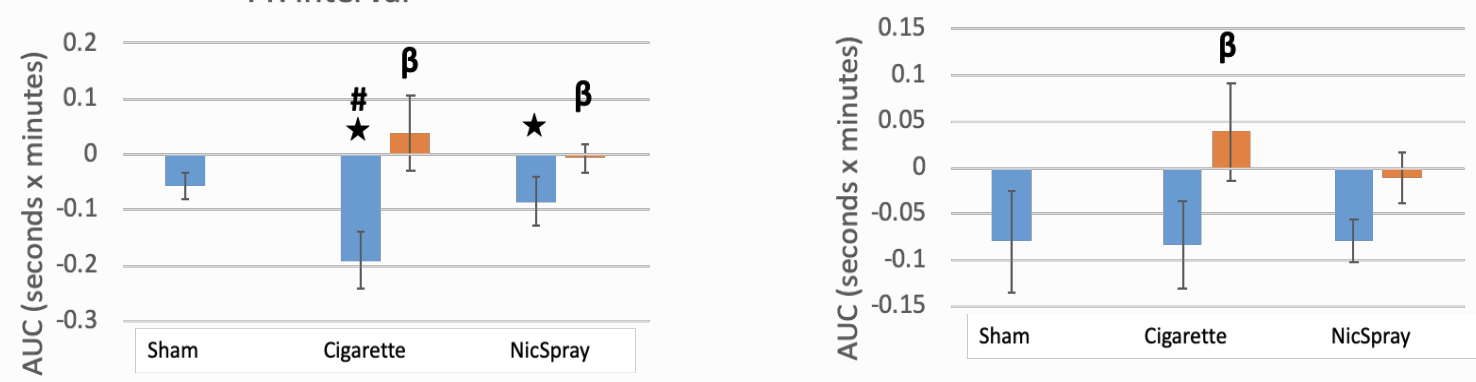

PR segment
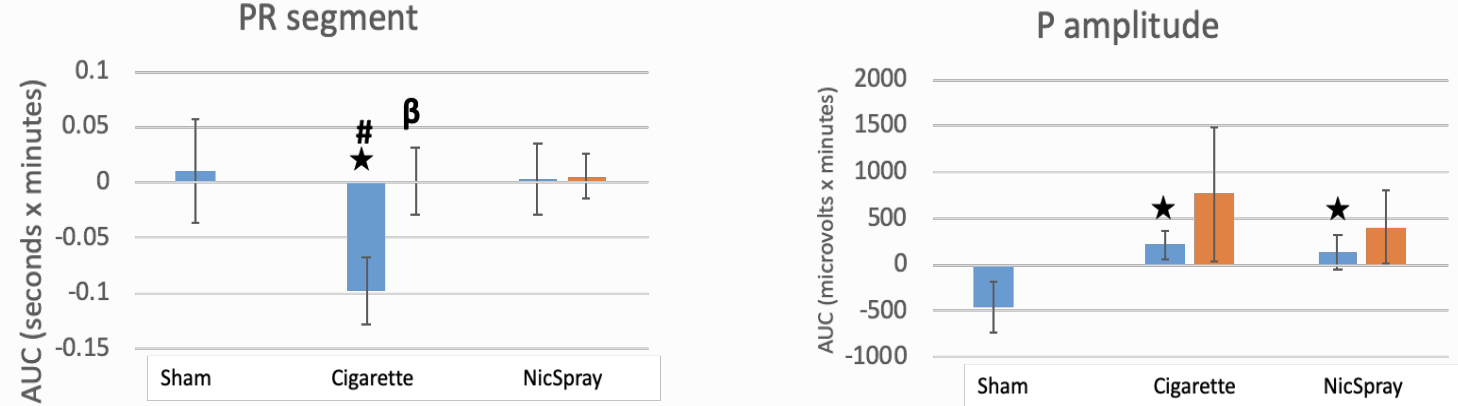


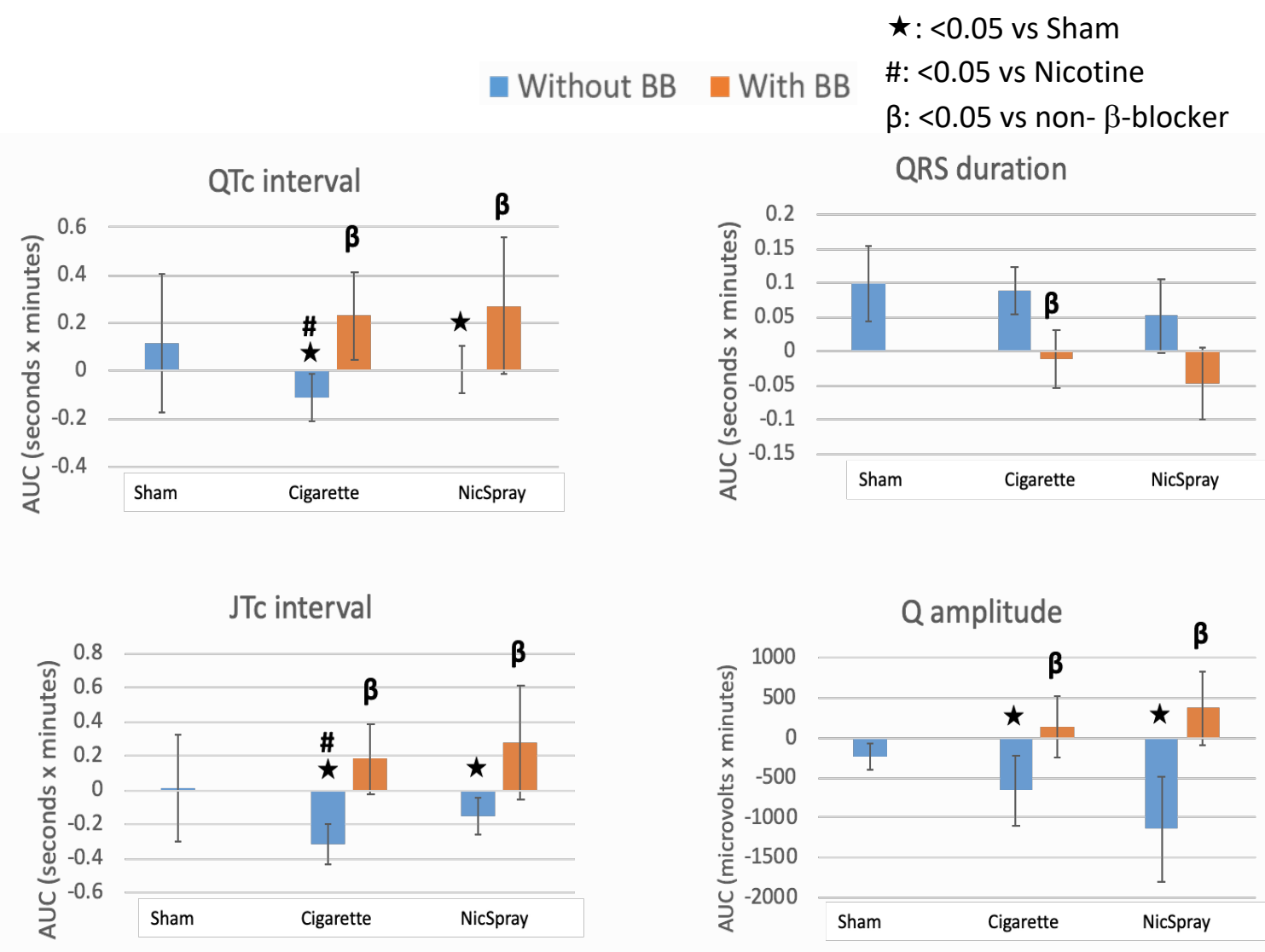

Figure 3.1 The Area Under the Curve for the raw changes $(\Delta)$ in ECG parameters from baseline for each visit day among smokers

$\mathrm{N}=20$ on Cigarette and NicSpray days. $\mathrm{N}=10$ on sham days 
Sham exposure

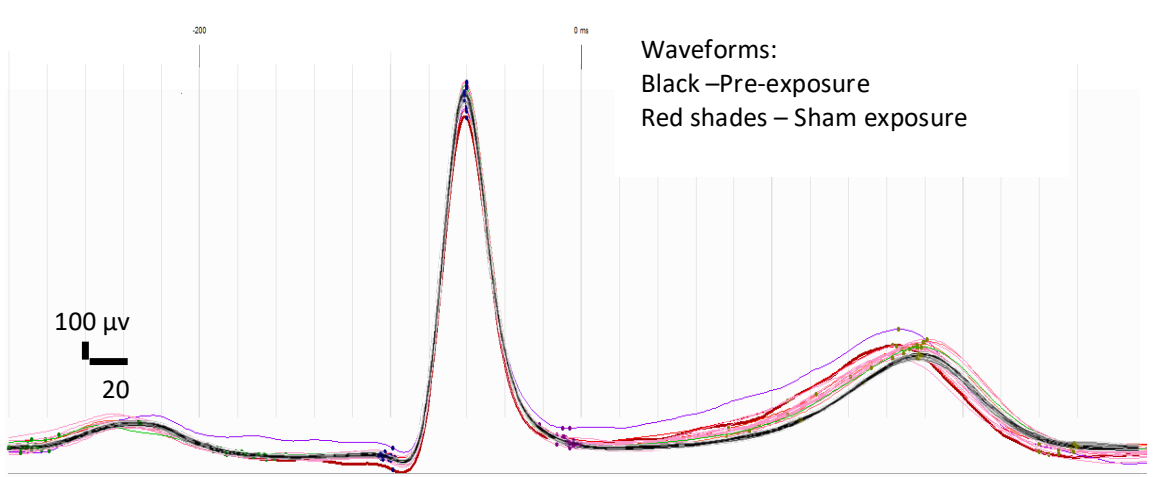

Cigarette exposure
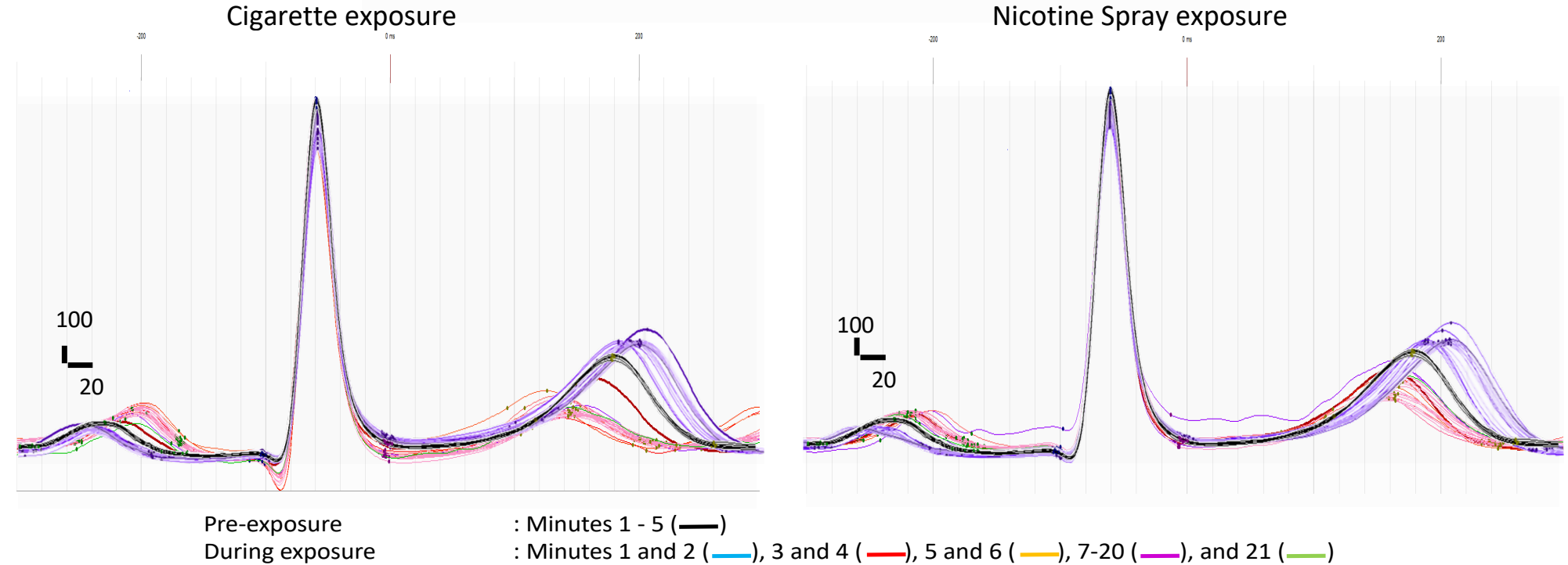

$\overline{8}$

Exposure $+\beta$-Blocker

:Minutes 1 and $2(-), 3$ and $4(-), 5$ and $6(-), 7-20(-)$, and $21(-)$

: Minutes 1-20 (-

Figure 3.2 ECG morphology in a typical participant during exposure on different days

Each ECG individual waveform is a 1-minute average before or during exposure to unlit cigarette (sham), cigarette, or nicotine spray. 
Cigarette

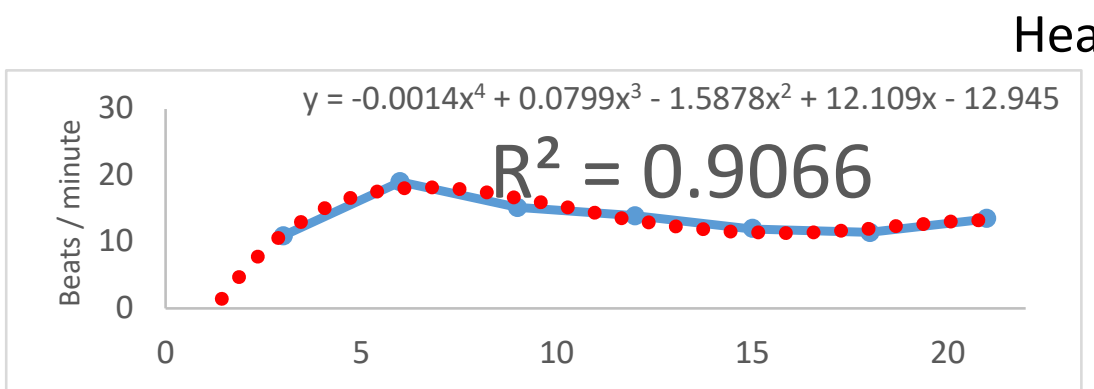

Nicotine Spray

Heart rate

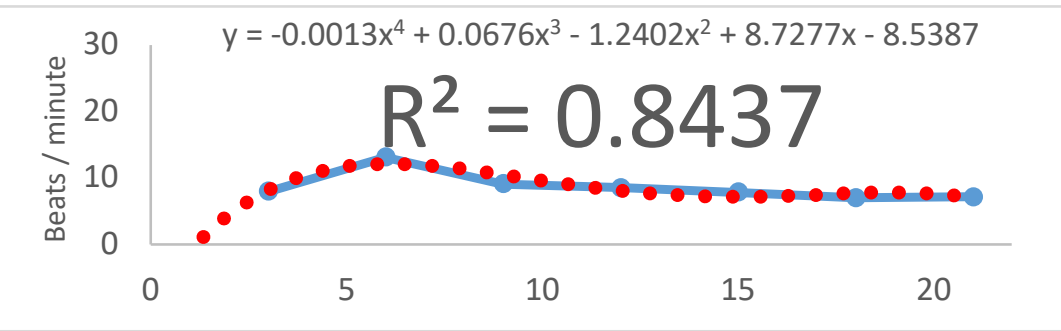

P amplitude
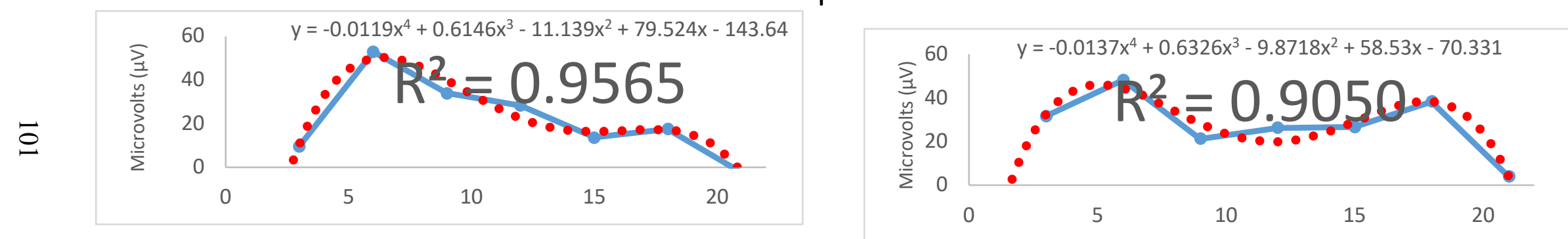

QT c

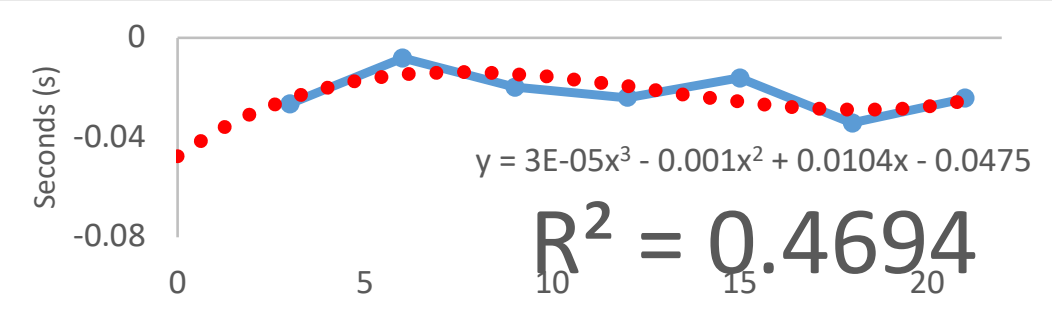



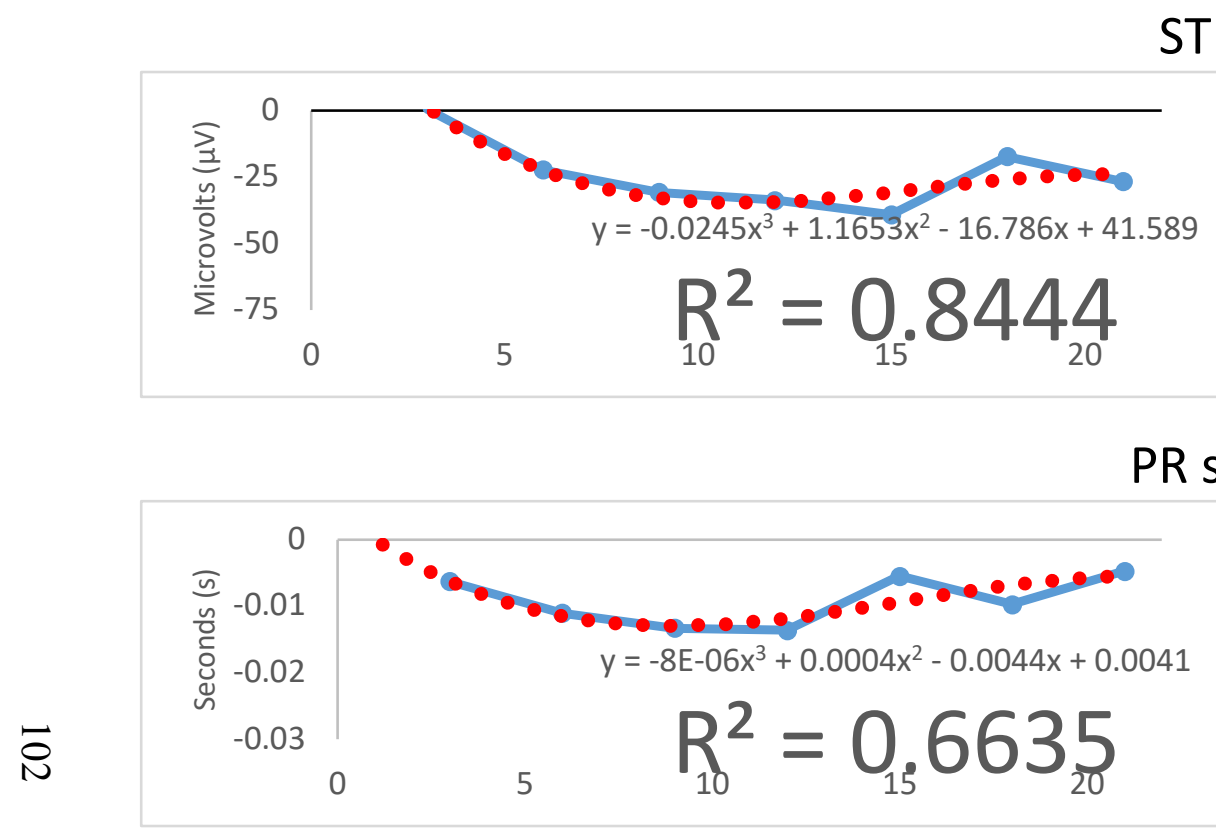

Figure 3.3 The best fitting line to predict changes in Heart Rate, P amplitude, and QTc upon Cigarette and Nicotine exposures, and additional lines for PR segment and ST height upon Cigarette exposure

$\mathrm{N}=20$ on Cigarette and NicSpray days. $\mathrm{N}=10$ on sham days 


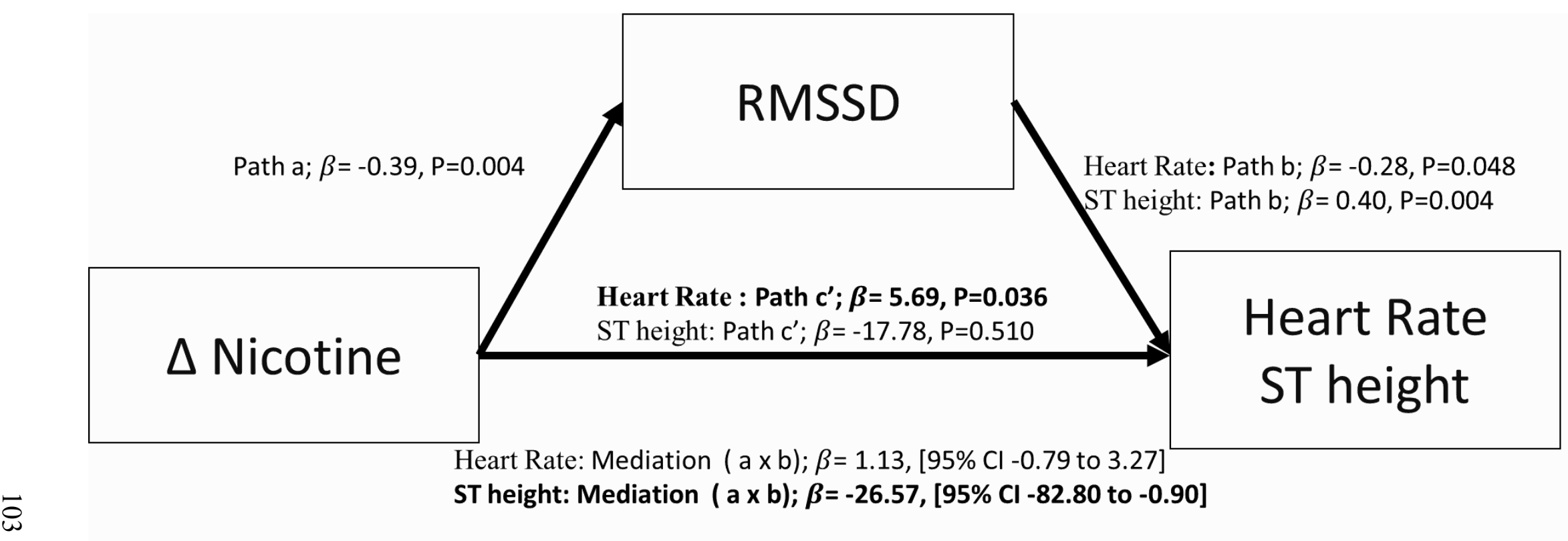

Figure 3.4 Mediation analyses of changes in Nicotine, root mean squared of successive differences (RMSSD) and Heart Rate and STheight among smokers on Cig-Day and NicS-Day and Non-smokers

Nicotine directly and linearly increases the heart rate, independent of pathway involving RMSSD / $\beta$-adrenoreceptors. RMSSD/ $\beta$-adrenoreceptors significantly and fully mediates the effect of smoking on ST height. 


\section{$\underline{\text { Discussion }}$}

We have demonstrated in healthy habitual smokers that either smoking one cigarette or self-administering $4 \mathrm{mg}$ oral nicotine acutely alters ECG morphology, with some key differences between the effects of the two. Moreover, non-selective $\beta$-adrenergic blockade inhibited many but not all of these effects. These transient ECG changes provide a plausible mechanism underlying smoking-induced cardiovascular events, with important clinical implications related to recreational use of nicotine delivery products as well. Table 3.2 summarizes the major findings of the study.

Table 3.2 Major findings of the experimental study

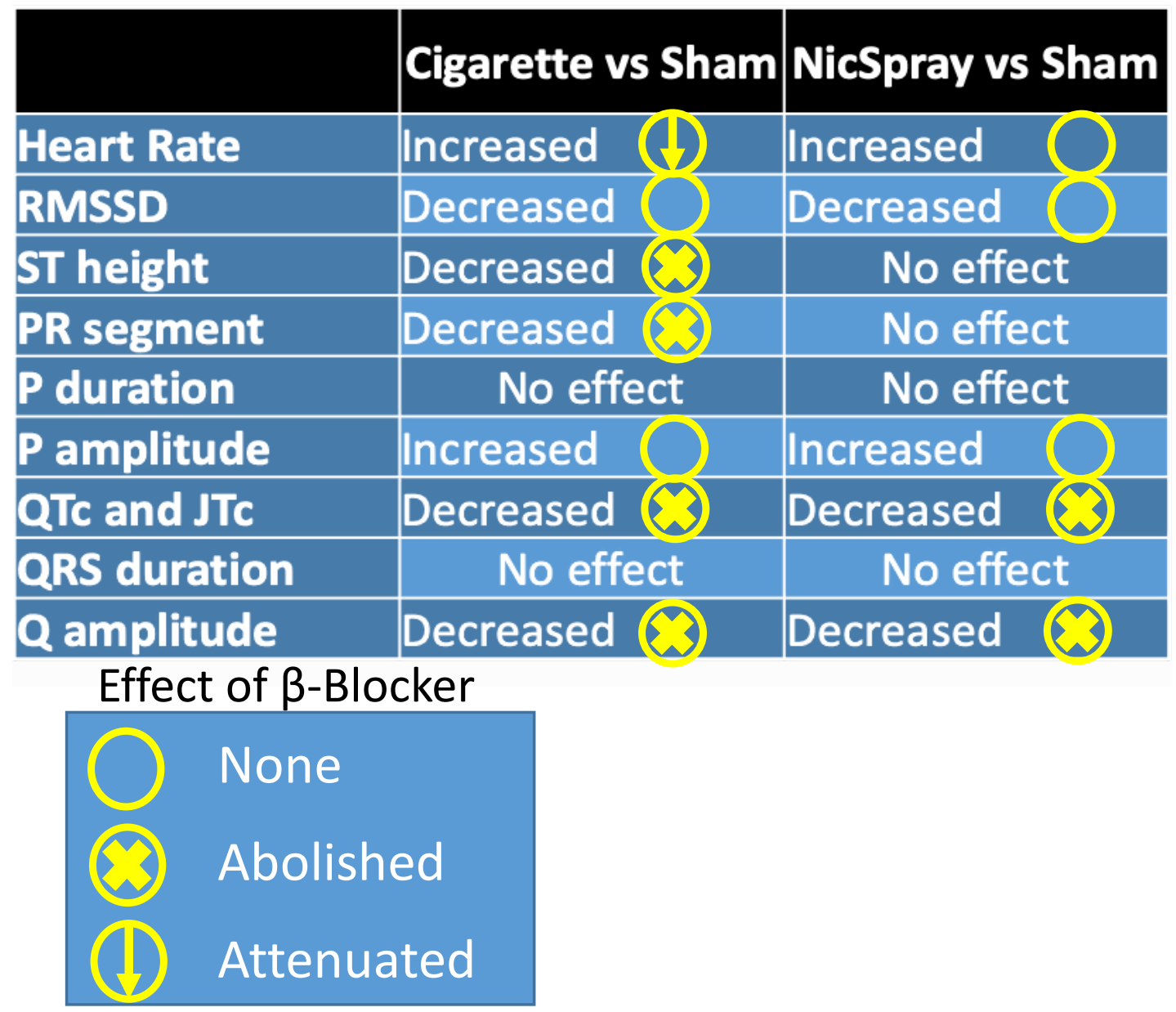




\section{Sympathetic activation}

Consistent with previous reports, our study found that smoking a single cigarette acutely increased heart rate and blood pressure, and decreased HRV and PR segment [90, $240,241,320]$ - consistent with increased sympathetic activation. The duration of increased heart rate up-to 20 minutes, dissipating after 30 minutes, recapitulates observations by Ramakrishnan et al, among 31 male smokers with atypical chest pain and $24 \mathrm{~h}$ Holter monitor [238]. However, we showed for the first time that acute nicotine exposure causes these changes, with dose-proportionate effects that were diminished by propranolol. Nicotine is a known sympathomimetic that acts on nicotinic receptors to increase sympathetic tone and catecholamine release. Thus, it is not surprising that we also found a dose-proportionate increase in cardiac sympathetic activity from oral nicotine administration. Our observations of nicotine-mediated sympatho-excitation add to recent literature on the acute physiological effects of nicotine alone [321-323] or within ecigarette aerosols [321] and identify a plausible mechanism by which tobacco product use increases sympathetic activity. Indeed, sympathetic dominance strongly predicts cardiovascular morbidity and mortality [303, 324, 325], including for arrhythmia-related events [326], and can acutely provoke arrhythmia through numerous pathways [327]. Importantly, our observations of acute sympathetic dominance with either nicotine oral spray or conventional smoking identify nicotine as a plausible mediator of tobacco productinduced cardiovascular morbidity and mortality. 
Atria

P wave morphology is dependent on mainly four factors 1) intra-atrial conduction abnormalities, 2) left atrial hypertension, 3) left atrial distention, and 4) chronicity disease [328]. Another novel finding of the study was that cigarette smoking and nicotine spray, within minutes, increased $\mathrm{P}$ wave amplitude, and propranolol prevented these effects. We also found non-significant shortening of $\mathrm{P}$ duration with cigarette and nicotine use. Both these observations are similar those of Goldberg et al., who noted that isoproterenolinduced $\beta$-adrenergic stimulation significantly shortened the $\mathrm{P}$ wave in healthy subjects [58]. Sympathetic stimulation increases peripheral vascular resistance, venous return and cardiac pressures [329], effects also seen with cigarette smoking [330, 331]. Accordingly, the significant increase in $\mathrm{P}$ amplitude induced by NicS and Cig, and inhibited by $\beta$ blockade, indicate that nicotine increases atrial pressures through $\beta$-adrenergic receptors. The acute increase in atrial pressures together with increased dromotropy, may represent two distinct pathways with possible synergistic effects of tobacco products induced increased atrial arrhythmia and thrombosis and stroke risk [283, 312].

\section{Ventricles}

To the best of our knowledge, this study demonstrates for the first time that smoking acutely induces ST-depression. Smoking caused a gradually worsening ST-depression that peaked at 9-12 minutes, abated by about 30 minutes post-exposure, and vanished with propranolol pretreatment. Recently, Ramakrishnan noted gross alterations in ST-T morphology with smoking (1-2 mm changes in ST segment or T wave), but only in one tenth of men with prior atypical chest pain and with onset at 8-12 minutes after smoking 
[238]. in [238]. The cardio-protective effects of $\beta$-blockers among patients with stable angina is well described [332]. However, cigarette smoking induced ST-segment depression at rest, via $\beta$-adrenergic receptors activation complements the propositions of others that enhanced sympathetic tone mediates ST-segment depression in general [333$335]$.

Interestingly, we also found that cigarette smoking caused significant albeit minimal shortening of QTc. The relationship between QTc and the ANS is particularly complex [299]. However, several studies have shown that acute adrenergic stimulation shortens QTc in humans [64, 336], possibly by reducing transmural dispersion of repolarization [337]. Chronic smoking is associated with higher potassium levels [338], however, acutely it inhibits potassium channels [85] and/or may decrease potassium levels via direct sympathetic activation [339]. The effects of potassium levels and/or ion channels may be more pronounced in the ventricles than in atria, resulting in hypokalemia related ECG changes and ST-segment depression [339]. Unlike the dose-dependent effects of nicotine (in either NicS or Cig) on atrial and atrioventricular (AV) electrophysiology, there were no significant effects of nicotine on ventricular depolarization and repolarization. We speculate this may derive from disproportionate autonomic control of the atria, sinus node, and $\mathrm{AV}$ node, which have a greater density of cardiac autonomic ganglia than the ventricles [340, 341]. Alternatively, because of distinct atrial-specific ion-channel and interceullular coupling properties and distribution, atrial myocardium may be more susceptible to architectural or structural changes with a substantial impact on cardiac performance, arrhythmia occurrence, and stroke risk [342]. 


\section{Strengths and Limitations}

It is well accepted that many of the effects of cigarette smoking, including addiction and acute alterations in cardiovascular physiology, stem from the rapid delivery of nicotine. However, there are very few studies that have directly compared the effects of nicotine vs cigarette, and those studies found no significant effect of nicotine replacement therapy, in contrast to cigarette exposure [343], we used a novel oral nicotine spray with pharmacokinetic properties for nicotine similar to those of cigarettes [314, 315, 344, 345], and also perhaps electronic nicotine delivery systems. We also recorded continuous standard 12 lead ECG during exposure, and systematically analyzed the interval and amplitudes of each beat with an automated software, to investigate the acute temporal relationship of smoking and nicotine exposure on ECG.

One potential source of variability in this study was the outdoor setting during midexposure ECG monitoring. However, to diminish the influence of outdoor conditions (e.g., temperature, humidity, air pollutants etc), most of the study visits were performed on consecutive days. Similarly, we saw no differences in ECG variables between BB and non-BB days during the first minute of outdoor recording. To facilitate detection of the early acute effects under typical smoking conditions and the delayed acute effects under conventional clinical conditions, participants were assessed for ECG changes while seated at mid-exposure, but while supine at pre- and post-exposure. We also performed the same protocol on 10 healthy non-smokers, where the exposure consisted of periodically inhaling through an empty straw for 5 minutes to simulate smoking, and demonstrated that there were minor changes related to position. We did not exclude any coronary artery disease among the study participants, however, because of our eligibility criteria, participants had 
no known cardiac medical condition, nor any active symptoms and were healthy. Another limitation is that the oral nicotine spray may be pungent and taste unpleasant for some participants, which may result in significant changes in heart rate. However, these changes are usually transient and less potent than that seen during our study (i.e., increase in heart rate of $7.1-13.6 \%$ for various taste stimuli, with maximum effect $\sim 25 \mathrm{~s}$ and returned to pre-exposure after 80 and 100 seconds [346]. Lastly, since this is a human in vivo experimental study, we were unable to differentiate direct vs indirect effects from nicotine and cigarette smoking (e.g., heart rate may have increased due to direct effects of nicotine and non-nicotine constituents on receptors in the sinoatrial node or central nervous system, or instead via reflexes from increased cardiac pressures secondary to increased peripheral vascular resistance and systemic pressures).

\section{$\underline{\text { Conclusion }}$}

Smoking and nicotine alone acutely induce myriad changes in electrophysiology that are known to be pro-arrhythmic. Smoking may acutely promote arrhythmia via nicotine-mediated acceleration of heart rate and ventricular repolarization $(\mathrm{QTc})$, with $\beta$ adrenoceptors mediating only the latter. Unlike acute nicotine, cigarette smoke rapidly depresses ST segment and shortens PR segment via $\beta$-adrenoceptor stimulation, indicating smoking transiently induces ischemia and accelerates dromotropy by sympathetic activation. Nicotine in cigarette smoke, via $\beta$-adrenoceptors, mostly seems responsible for transient increase in atrial pressures, which may reflect possible mechanism for tobacco induced atrial arrhythmia and stroke risk. 


\section{CHAPTER IV \\ IMPLICATIONS AND CONCLUSIONS}

\section{$\underline{\text { Clinical Implications }}$}

Cigarette smoking is the most significant modifiable risk factor for cardiovascular morbidity and mortality. Although smoking rates in the United States have declined over the past five decades to historic lows, efforts to protect public health are far from complete. The burden of combustible tobacco use in the United States remains high, especially in vulnerable populations. This persistence of cigarette smoking, coupled with the advent and dramatic rise in the use of new tobacco products, such as electronic cigarettes, especially among adolescents and young adults, are of significant concern [347]. These products may benefit by helping some smokers quit or transition to a less harmful product; however, the long-term health effects of these products and the net public health effect associated with their use remain unclear and widely debated [348]. Evidence is mounting that the use of these products may catalyze the transition to the use of other tobacco products or recreational drugs, particularly in young adults [349]. The use of certain newly regulated tobacco products has skyrocketed, particularly among youths. For example, from 2011 to 2018 , the rate of e-cigarette use among high school students ballooned from $1.5 \%$ to $20.8 \%$ [350], and then up to $27.5 \%$ in 2019 , according to preliminary results from the Centers for Disease Control and Prevention's annual National Youth Tobacco Survey. 
Nicotine is addictive and present alongside other chemicals in ENDS. Several studies have revealed the existence of toxicants in both the liquid and aerosol of ecigarettes. Recent studies suggest that, although adverse experiences such as chest pain, palpitations, coronary heart disease, lung injury [351], and increased risk of myocardial infarction [352] are associated with e-cigarette use, further investigation is required to evaluate whether any causal relationships exist. The FDA has included the discovery of biomarkers to assess exposure and harm or toxicity of non-cigarette tobacco products, including ENDS, as well the as short- and long-term health effects of tobacco products among its research priorities [348]. The series of studies presented in this dissertation directly relate to those scientific domains - to help establish sensitive biomarkers to detect short-and long-term subclinical cardiovascular injury from tobacco and other nicotinerelated products, and potentially guide regulation of nicotine levels in recreational products [347].

Most investigations into the pharmacokinetics of nicotine from ENDS have yielded variable results, perhaps owing to the large variability of study protocols. Moreover, several studies have lacked generalizability, as they incorporated a single standard ecigarette and nicotine concentrations with a protocol for vaping at fixed intervals [353]. Furthermore, they were unable to investigate the differential impacts of variations in experimental parameters, such as battery output voltage or coil resistance, which are known to impact delivery of nicotine and production of potentially toxic constituents [354-356]. Further complicating the relevance of past ENDS studies, e-cigarette devices have rapidly evolved to more effectively deliver nicotine. A recent pharmacokinetic study revealed that an ad-lib session with third-generation e-cigarettes is able to achieve the dose and speed of 
nicotine delivery similar to conventional cigarettes [357]. Another study revealed that newer generation e-cigarettes ("mod"-type cigarette) delivered nicotine at levels close to, or even exceeding, those of combustible cigarettes (highest average Cmax - $43.6 \mathrm{ng} / \mathrm{ml}$ ) [358]. The parent company of JUUL, the relatively new pod device that has claimed 70\%$80 \%$ of the U.S. e-cigarette market [359], noted in their initial patent filing that their device achieved 36\% higher peak levels of plasma nicotine and higher increases in heart rate with more rapid onset than full-flavored cigarettes. This is due in major part to the use of nicotine benzoic acid salts, which further enhance nicotine delivery [359]. In our experimental trial, the plasma levels were drawn about 45 minutes after the start of exposure, and the nicotine levels increased an average of $7.83 \mathrm{ng} / \mathrm{ml}$ after cigarette use, and $6.98 \mathrm{ng} / \mathrm{ml}$ after nicotine spray, which is similar to that achieved by newer e-cigarettes [353]. The JUUL brand is one of the newer entries into the market and utilizes prefilled EC fluid "pods," being more similar to the "cig-a-like" products than to the recently available tank/box mod styles. Nicotine concentrations (average $60.9 \mathrm{mg} / \mathrm{mL}$ ) were significantly higher in JUUL than those of any EC products previously analyzed by Omaiye et al. [360]. Such high nicotine levels are not observed after use of NRTs, with doses recommended to reduce withdrawal symptoms and cigarette cravings [344]. Despite the large variability in the pharmacokinetic profiles and levels of nicotine due to varying e-cigarette device designs, e-liquid concentration, and use behavior, the results of our study provide valuable insight into the likely cardiovascular effects and risks of e-cigarettes.

We conducted three studies that may elucidate the cardiac impacts of nicotinecontaining e-cigarettes. In our cross-sectional observational study, we found that chronic nicotine or tobacco exposure leads to dose-dependent shortening of PR segment and QTc, 
and that an extremely high burden of exposure (measured by the nicotine metabolite, cotinine) leads to QRS shortening and JT prolongation. Although our analyses suggested a role for nicotine in these effects, the design of this study made it impossible to delineate between effects from exposure to cigarette smoke and exposure to nicotine. In our followup acute exposure study, we found evidence that nicotine mediates increased sympathetic activity (as measured by decreased HRV), shortened JTc, and increased P amplitude. Taking these findings into consideration - and extending to the possible nicotine-related effects from ENDS on cardiac electrophysiology—we suggest that e-cigarettes both acutely and chronically increase sympathetic activity, as noted in recent observations [321, 361]. Other studies have also found that conventional smoking chronically increases sympathetic modulation of the heart $[72,241]$. Moreover, our results suggest that nicotine in e-cigarettes may both acutely and chronically shorten ventricular repolarization while also acutely increasing atrial pressures (indicated by P amplitude). Notably, a few studies also observed an increased P amplitude among smokers at baseline [212, 213, 362, 363], similar to our findings in which cotinine (proxy for tobacco use) was associated with $\mathrm{P}$ amplitude (particularly in deep terminal negativity of the P wave in V1 [DTNPV1]) in the chronic state [364]. However, the mechanisms for abnormal P wave indices during acute and chronic exposure may differ while also carrying different prognostic value. As acute increases in P amplitude may reflect increased cardiac filling pressure, chronic elevations in P amplitude may reflect atrial hypertrophy/distention.

Such baseline pathophysiological changes in human cardiac electrophysiology have clinically important implications. Increases in sympathetic activity [303], atrial cardiopathy [365, 366], and shortened rate of ventricular repolarization [367-369] have 
been associated with increased risk for adverse outcomes, such as all-cause mortality, arrhythmia, and stroke. In this regard, a strategy formed by the FDA to limit nicotine levels in ENDS might help reduce some of the long-term harmful cardiovascular effects. Our study featuring oral nicotine spray indicated that the nicotine levels should be lower than those currently found in newer generation e-cigarettes (e.g., JUUL and other pod devices). However, more studies are required to establish whether the nicotine levels are directly related to increased risk for short- or long-term adverse cardiovascular events.

\section{$\underline{\text { Conclusion }}$}

The acute and chronic proarrhythmic effects of smoking and their underlying mechanisms are currently unknown. To address this gap in the knowledge, we assessed the effects of chronic cigarette exposure on ECG, using serum cotinine (as a sensitive and specific marker of tobacco use) from the NHANES database, and urinary cotinine from cardiovascular patients from an outpatient clinic. From the NHANES database, higher serum cotinine levels were associated with considerably shortened PR intervals, PR segments, and QRS duration, and considerably prolonged JT intervals. We also found a negative linear relationship between cotinine and QTc, as well as between cotinine and PR segment. A similar effect on atrial electrocardiographic indices (shortened PR interval and PR segment) was also observed among the CVD patients. Interestingly, our statistical mediation analyses indicated that dopamine fully mediated the association between

cotinine and the shortening of both PR intervals and segments. Collectively, the results from the chronic exposure studies suggest that habitual smoking accelerates atrioventricular conduction (possibly though dopamine) and ventricular depolarization, but 
appears to exert a more complex effect on repolarization. Overall habitual smoking modestly accelerates ventricular repolarization, and simultaneously increases the risk for severe slowing of ventricular repolarization in a subset of patients.

An experimental trial to investigate $\beta$-adrenergic receptors' role in the acute effects of smoking versus nicotine spray on ECG was also performed. Nicotine, both with and without a $\beta$-blocker, increases the sympathetic nervous activity to a similar extent as observed with cigarette smoking in the presence of a $\beta$-blocker. Nicotine increases the heart rate through receptors independent of the $\beta$-adrenergic receptors, suggesting its acute and direct action on cardiomyocytes is independent of increased central sympathetic activation. Cigarette smoking had a higher effect on heart rate; however, after treatment with a $\beta$ blocker, the heart rate increase was similar to that from nicotine (with or without a $\beta$ blocker). This suggests that the non-nicotine constituents of cigarette smoke that increase heart rate act through $\beta$-adrenergic receptors. Cigarette smoking and nicotine comparably increase P-wave amplitude without affecting P duration (which was also unaffected in chronic exposure studies), and $\beta$-blockade did not alter this effect. This increase in $\mathrm{P}$ amplitude may derive from increased peripheral vascular resistance with both exposures, which increases cardiac filling pressures and cardiac output. Cigarette smoking, but not nicotine, also shortened the PR segment and decreased the height of the ST segment, with both being rescued by $\beta$-blockade. This suggests that non-nicotine constituents increase dromotropy and ischemia through $\beta$-adrenergic receptors. Furthermore, the relationship between cotinine and the PR segment found in the chronic exposure studies likely derived from cotinine reflecting exposure to tobacco smoke, including non-nicotine constituents. Indeed, the tar and nicotine levels in cigarettes correspond with one another, just as 
exposures to each would be expected to correspond. Consistent with our observations of the link between chronic smoking and QTc shortening, ventricular repolarization time was more acutely shortened with cigarette smoking compared to nicotine, and was abolished by the $\beta$-blockade. It is plausible that nicotine acutely accelerates repolarization via adrenergic-related mechanisms (e.g., $\beta$-adrenergic induced phosphorylation of Kv7.1), whereas chronic smoking, itself, impedes repolarization via myocardial injury (e.g., fibrosis). Alternatively, chronic smoking may impede repolarization by desensitizing adrenergic pathways that would otherwise modulate the heart-rate-dependent shortening of repolarization with sympathetic activation in healthy individuals.

The central illustration combines the findings of all three studies to demonstrate the role of nicotine and non-nicotine constituents of cigarette smoke, and the pathways involved in their effects on cardiac electrophysiology (Figure 4.1). 


\section{Nicotine alone Smoking}

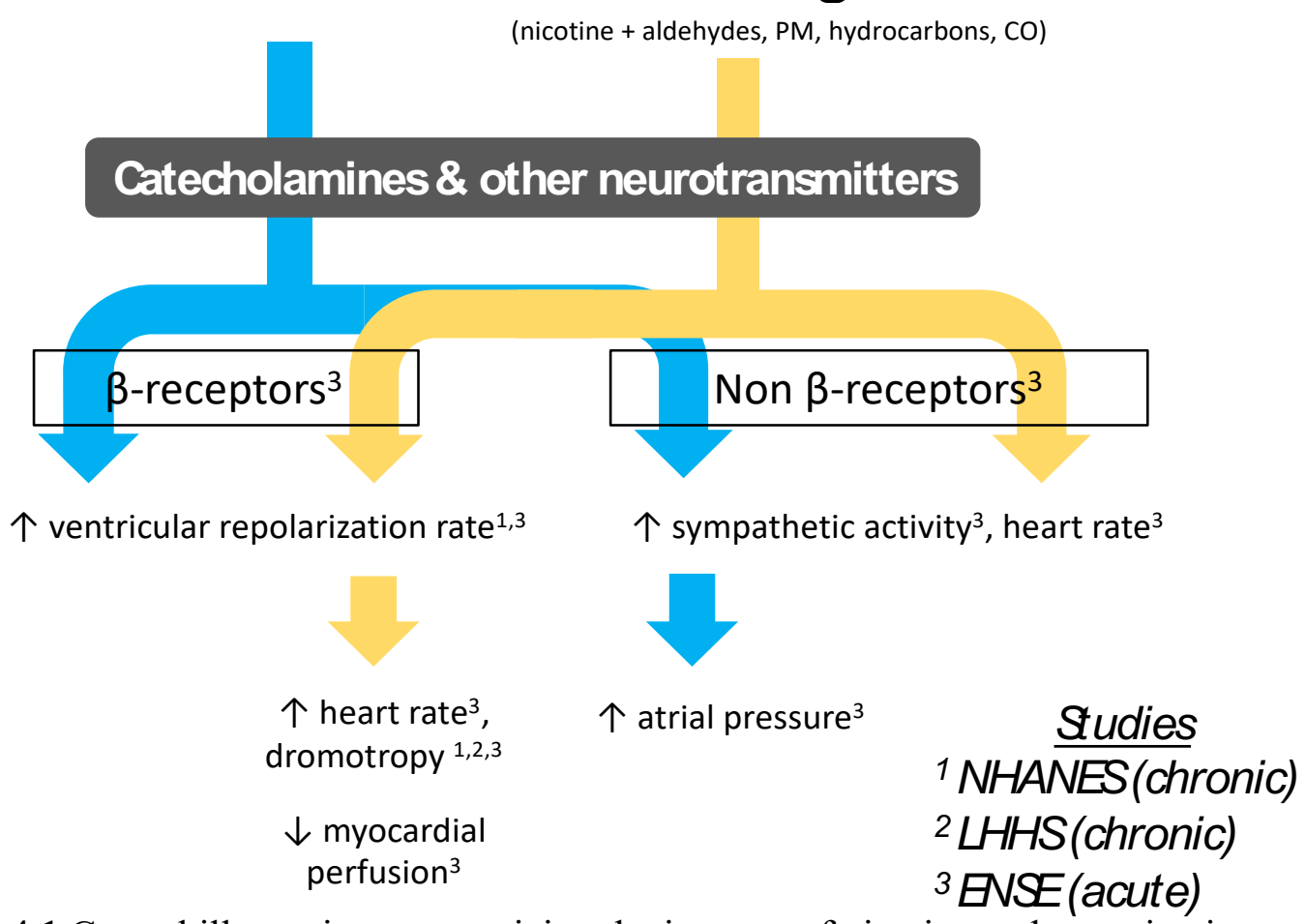

Figure 4.1 Central illustration summarizing the impacts of nicotine and non-nicotine constituents of cigarette smoke on cardiac electrophysiology, and the pathways likely involved

Key summary points

- Of the acute effects of cigarette smoking on ECG, nicotine is partly responsible for increased sympathetic activity and shortening of ventricular repolarization, but not for increased dromotropy and ischemia.

- Habitual smoking is associated with increased dromotropy (possibly via dopamine), faster ventricular depolarization, and inverse J-shaped effects on ventricular repolarization. 
- $\quad$ Such acute and chronic effects on ECG from cigarette smoking and nicotine may promote cardiac arrhythmias or reflect a diseased state and an at-risk population.

- $\quad$ Future research is needed to (a) delineate the receptors used by nicotine to cause sympathetic activation, (b) identify the non-nicotine constituents in cigarette smoke that transiently increase dromotropy and myocardial ischemia, and (c) elucidate the mechanisms involved in nicotine and non-nicotine constituents of cigarette-smokeinduced complex effects on ventricular repolarization (shortening in most patients, and prolonging in a small subset of patients). 


\section{REFERENCES}

1. General., A.R.o.t.S., The health consequences of smoking - 50 years of progress: a report of the Surgeon General. . 2014: Washington, DC.

2. Burns, D.M., Epidemiology of smoking-induced cardiovascular disease. Prog Cardiovasc Dis, 2003. 46(1): p. 11-29.

3. Centers for Disease, C. and Prevention, Smoking-attributable mortality, years of potential life lost, and productivity losses--United States, 2000-2004. MMWR Morb Mortal Wkly Rep, 2008. 57(45): p. 1226-8.

4. Smoking and Mortality - Beyond Established Causes. N Engl J Med, 2016. 375(24): p. 2410.

5. Jha, P., et al., 21st-century hazards of smoking and benefits of cessation in the United States. N Engl J Med, 2013. 368(4): p. 341-50.

6. Bjartveit, K. and A. Tverdal, Health consequences of smoking 1-4 cigarettes per day: response to G F Cope (eletter to journal). Tob Control, 2006. 15(1): p. 71-2.

7. Xu, X., et al., Annual healthcare spending attributable to cigarette smoking: an update. Am J Prev Med, 2015. 48(3): p. 326-33.

8. Jamal, A., et al., Current Cigarette Smoking Among Adults - United States, 2016. MMWR Morb Mortal Wkly Rep, 2018. 67(2): p. 53-59.

9. Benowitz, N.L., Nicotine addiction. N Engl J Med, 2010. 362(24): p. 2295-303.

10. Mansvelder, H.D. and D.S. McGehee, Long-term potentiation of excitatory inputs to brain reward areas by nicotine. Neuron, 2000. 27(2): p. 349-57.

11. George, O., et al., CRF-CRF1 system activation mediates withdrawal-induced increases in nicotine self-administration in nicotine-dependent rats. Proc Natl Acad Sci U S A, 2007. 104(43): p. 17198-203.

12. Grunberg, N.E., A neurobiological basis for nicotine withdrawal. Proc Natl Acad Sci U S A, 2007. 104(46): p. 17901-2.

13. Bhatnagar, A., et al., Electronic cigarettes: a policy statement from the American Heart Association. Circulation, 2014. 130(16): p. 1418-36.

14. Grana, R., N. Benowitz, and S.A. Glantz, E-cigarettes: a scientific review. Circulation, 2014. 129(19): p. 1972-86.

15. Keith, R.J., et al., Characterization of Volatile Organic Compound (VOC) metabolites in Cigarette smokers, Electronic Nicotine Device Users, Dual Users and Non- users of tobacco. Nicotine Tob Res, 2019. 
16. Glantz, S.A. and D.W. Bareham, E-Cigarettes: Use, Effects on Smoking, Risks, and Policy Implications. Annu Rev Public Health, 2018. 39: p. 215-235.

17. in How Tobacco Smoke Causes Disease: The Biology and Behavioral Basis for Smoking-Attributable Disease: A Report of the Surgeon General. 2010: Atlanta (GA).

18. Arredondo, J., et al., Receptor-mediated tobacco toxicity: regulation of gene expression through alpha3beta2 nicotinic receptor in oral epithelial cells. Am J Pathol, 2005. 166(2): p. 597-613.

19. Ambrose, J.A. and R.S. Barua, The pathophysiology of cigarette smoking and cardiovascular disease: an update. J Am Coll Cardiol, 2004. 43(10): p. 1731-7.

20. Balakumar, P. and J. Kaur, Is nicotine a key player or spectator in the induction and progression of cardiovascular disorders? Pharmacol Res, 2009. 60(5): p. 361-8.

21. Klabunde, R.E., Cardiac electrophysiology: normal and ischemic ionic currents and the ECG. Adv Physiol Educ, 2017. 41(1): p. 29-37.

22. Kligfield, P., et al., Recommendations for the standardization and interpretation of the electrocardiogram: part I: the electrocardiogram and its technology a scientific statement from the American Heart Association Electrocardiography and Arrhythmias Committee, Council on Clinical Cardiology; the American College of Cardiology Foundation; and the Heart Rhythm Society endorsed by the International Society for Computerized Electrocardiology. J Am Coll Cardiol, 2007. 49(10): p. 1109-27.

23. Mozos, I. and A. Caraba, Electrocardiographic Predictors of Cardiovascular Mortality. Dis Markers, 2015. 2015: p. 727401.

24. Denes, P., et al., Major and minor ECG abnormalities in asymptomatic women and risk of cardiovascular events and mortality. JAMA, 2007. 297(9): p. 978-85.

25. Auer, R., et al., Association of major and minor ECG abnormalities with coronary heart disease events. JAMA, 2012. 307(14): p. 1497-505.

26. Soliman, E.Z., M. Cammarata, and Y. Li, Explaining the inconsistent associations of PR interval with mortality: the role of P-duration contribution to the length of PR interval. Heart Rhythm, 2014. 11(1): p. 93-8.

27. Smith, J.W., et al., PR-Interval Components and Atrial Fibrillation Risk (from the Atherosclerosis Risk in Communities Study). Am J Cardiol, 2017. 119(3): p. 466472.

28. Magnani, J.W., et al., $P$ wave indices, obesity, and the metabolic syndrome: the atherosclerosis risk in communities study. Obesity (Silver Spring), 2012. 20(3): p. 666-72.

29. Rasmussen, P.V., et al., Electrocardiographic PR Interval Duration and Cardiovascular Risk: Results From the Copenhagen ECG Study. Can J Cardiol, 2017. 33(5): p. 674-681. 
30. Bang, C.N. and P.M. Okin, The long and short of the PR-interval: relation to cardiovascular outcome in patients with coronary heart disease. Europace, 2015. 17(6): p. 838-9.

31. Crow, R.S., P.J. Hannan, and A.R. Folsom, Prognostic significance of corrected $Q T$ and corrected JT interval for incident coronary heart disease in a general population sample stratified by presence or absence of wide QRS complex: the ARIC Study with 13 years of follow-up. Circulation, 2003. 108(16): p. 1985-9.

32. Zulqarnain, M.A., et al., Risk of Mortality Associated With QT and JT Intervals at Different Levels of QRS Duration (from the Third National Health and Nutrition Examination Survey). Am J Cardiol, 2015. 116(1): p. 74-8.

33. Rosenthal, T.M., et al., T-peak to T-end interval for prediction of ventricular tachyarrhythmia and mortality in a primary prevention population with systolic cardiomyopathy. Heart Rhythm, 2015. 12(8): p. 1789-97.

34. Panikkath, R., et al., Prolonged Tpeak-to-tend interval on the resting ECG is associated with increased risk of sudden cardiac death. Circ Arrhythm Electrophysiol, 2011. 4(4): p. 441-7.

35. Erikssen, G., et al., The terminal part of the QT interval (T peak to T end): $a$ predictor of mortality after acute myocardial infarction. Ann Noninvasive Electrocardiol, 2012. 17(2): p. 85-94.

36. Ashley, E.A., V. Raxwal, and V. Froelicher, An evidence-based review of the resting electrocardiogram as a screening technique for heart disease. Prog Cardiovasc Dis, 2001. 44(1): p. 55-67.

37. Kianoush, S., et al., Associations of Cigarette Smoking With Subclinical Inflammation and Atherosclerosis: ELSA-Brasil (The Brazilian Longitudinal Study of Adult Health). J Am Heart Assoc, 2017. 6(6).

38. Abrams, D.B., et al., Harm Minimization and Tobacco Control: Reframing Societal Views of Nicotine Use to Rapidly Save Lives. Annu Rev Public Health, 2018. 39: p. 193-213.

39. Administration, F.a.D., Tobacco product standard for nicotine level of combusted cigarettes: a proposed rule. 2018: Fed Reg. p. 11818-11843.

40. Zeller, M., The Future of Nicotine Regulation: Key Questions and Challenges. Nicotine Tob Res, 2019. 21(3): p. 331-332.

41. Apelberg, B.J., et al., Potential Public Health Effects of Reducing Nicotine Levels in Cigarettes in the United States. N Engl J Med, 2018. 378(18): p. 1725-1733.

42. Hatsukami, D.K. and E.C. Donny, The Debate About Nicotine Addiction and the Role of Medicinal Products: Commentary on Zeller. Nicotine Tob Res, 2019. 21(3): p. 338-339.

43. Armour, J.A., Potential clinical relevance of the 'little brain' on the mammalian heart. Exp Physiol, 2008. 93(2): p. 165-76. 
44. Shen, M.J. and D.P. Zipes, Role of the autonomic nervous system in modulating cardiac arrhythmias. Circ Res, 2014. 114(6): p. 1004-21.

45. Ali, I.M., et al., Modification of supraventricular tachyarrhythmias by stimulating atrial neurons. Ann Thorac Surg, 1990. 50(2): p. 251-6.

46. Tomaselli, G.F., Mechanisms for Altered Autonomic and Oxidant Regulation of Cardiac Sodium Currents in Cardiac Electrophysiology: From Cell to Bedside, D.P.J. Zipes, José; Stevenson, William G.,, Editor. 2018, ELSEVIER. p. 362-369.

47. Shibata, E.F., et al., Autonomic regulation of voltage-gated cardiac ion channels. J Cardiovasc Electrophysiol, 2006. 17 Suppl 1: p. S34-S42.

48. Ono, K., H.A. Fozzard, and D.A. Hanck, Mechanism of cAMP-dependent modulation of cardiac sodium channel current kinetics. Circ Res, 1993. 72(4): p. 807-15.

49. Matsuda, J.J., H.C. Lee, and E.F. Shibata, Acetylcholine reversal of isoproterenolstimulated sodium currents in rabbit ventricular myocytes. Circ Res, 1993. 72(3): p. $517-25$.

50. Carll, A.F.A., Roberts AM, The Role of the Autonomic Nervous System in Cardiovascular Toxicity, in Comprehensive Toxicology, C.A. McQueen, Editor. 2018, Elsevier. p. 61-114.

51. Schubert, B., et al., Beta-adrenergic inhibition of cardiac sodium channels by dual G-protein pathways. Science, 1989. 245(4917): p. 516-9.

52. Grant, A.O., Cardiac ion channels. Circ Arrhythm Electrophysiol, 2009. 2(2): p. 185-94.

53. Lundby, A. and J.V. Olsen, Phosphoproteomics taken to heart. Cell Cycle, 2013. 12(17): p. 2707-8.

54. Dvir, M., et al., Long QT mutations at the interface between KCNQ1 helix $C$ and KCNE1 disrupt I(KS) regulation by PKA and PIP(2). J Cell Sci, 2014. 127(Pt 18): p. 3943-55.

55. Kang, C., et al., beta-adrenergic stimulation augments transmural dispersion of repolarization via modulation of delayed rectifier currents IKs and IKr in the human ventricle. Sci Rep, 2017. 7(1): p. 15922.

56. Moreno-Galindo, E.G., et al., Pharmacological Conversion of a Cardiac Inward Rectifier into an Outward Rectifier Potassium Channel. Mol Pharmacol, 2016. 90(3): p. 334-40.

57. Liang, B., et al., G-protein-coupled inward rectifier potassium current contributes to ventricular repolarization. Cardiovasc Res, 2014. 101(1): p. 175-84.

58. Cheema, A.N., et al., Effects of autonomic stimulation and blockade on signalaveraged $P$ wave duration. J Am Coll Cardiol, 1995. 26(2): p. 497-502.

59. Nollo, G., et al., Evidence of low-and high-frequency oscillations in human AV interval variability: evaluation with spectral analysis. Am J Physiol, 1994. 267(4 Pt 2): p. H1410-8. 
60. Kowallik, P. and M. Meesmann, Independent autonomic modulation of the human sinus and AV nodes: evidence from beat-to-beat measurements of $P R$ and $P P$ intervals during sleep. J Cardiovasc Electrophysiol, 1995. 6(11): p. 993-1003.

61. Wallace, A.G. and S.J. Sarnoff, Effects of Cardiac Sympathetic Nerve Stimulation on Conduction in the Heart. Circ Res, 1964. 14: p. 86-92.

62. Goldberger, J.J., et al., Assessment of effects of autonomic stimulation and blockade on the signal-averaged electrocardiogram. Circulation, 1994. 89(4): p. 1656-64.

63. Folino, A.F., et al., The effects of sympathetic stimulation induced by mental stress on signal-averaged electrocardiogram. Int J Cardiol, 1995. 48(3): p. 27985.

64. Algra, A., et al., Effect of beta-blockers on the relation between QT-interval and heart rate in exercise ECG. Eur Heart J, 1987. 8 Suppl D: p. 71-3.

65. Cuomo, S., et al., Influence of autonomic tone on QT interval duration. Cardiologia, 1997. 42(10): p. 1071-6.

66. Magnano, A.R., et al., Autonomic nervous system influences on QT interval in normal subjects. J Am Coll Cardiol, 2002. 39(11): p. 1820-6.

67. Li, Y.F., C. LaCroix, and J. Freeling, Specific subtypes of nicotinic cholinergic receptors involved in sympathetic and parasympathetic cardiovascular responses. Neurosci Lett, 2009. 462(1): p. 20-3.

68. Li, Y.F., C. Lacroix, and J. Freeling, Cytisine induces autonomic cardiovascular responses via activations of different nicotinic receptors. Auton Neurosci, 2010. 154(1-2): p. 14-9.

69. Neff, R.A., et al., Nicotine enhances presynaptic and postsynaptic glutamatergic neurotransmission to activate cardiac parasympathetic neurons. Circ Res, 1998. 83(12): p. 1241-7.

70. Kirpekar, S.M., A.G. Garcia, and J.C. Prat, Action of nicotine on sympathetic nerve terminals. J Pharmacol Exp Ther, 1980. 213(1): p. 133-8.

71. Haass, M. and W. Kubler, Nicotine and sympathetic neurotransmission. Cardiovasc Drugs Ther, 1997. 10(6): p. 657-65.

72. Middlekauff, H.R., J. Park, and R.S. Moheimani, Adverse effects of cigarette and noncigarette smoke exposure on the autonomic nervous system: mechanisms and implications for cardiovascular risk. J Am Coll Cardiol, 2014. 64(16): p. 174050.

73. Gerber, S.H., et al., Role of $[\mathrm{Na}+] \mathrm{i}$ and $[\mathrm{Ca} 2+] \mathrm{i}$ in nicotine-induced norepinephrine release from bovine adrenal chromaffin cells. Am J Physiol, 1995. 269(3 Pt 1): p. C572-81.

74. Wada, A., et al., Influx of $22 \mathrm{Na}$ through acetylcholine receptor-associated $\mathrm{Na}$ channels: relationship between 22Na influx, 45Ca influx and secretion of 
catecholamines in cultured bovine adrenal medulla cells. Neuroscience, 1985. 15(1): p. 283-92.

75. Kruger, C., et al., Nicotine-induced exocytotic norepinephrine release in guineapig heart, human atrium and bovine adrenal chromaffin cells: modulation by single components of ischaemia. J Mol Cell Cardiol, 1995. 27(8): p. 1491-506.

76. Li, Q. and E.J. Forsberg, Catecholamine secretion induced by nicotine is due to $\mathrm{Ca}++$ channel but not $\mathrm{Na}+$ channel activation in porcine adrenal chromaffin cells. J Pharmacol Exp Ther, 1996. 277(3): p. 1209-14.

77. Abajo, F.J., et al., Sodium-dependent and sodium-independent nicotine-evoked catecholamine release from cat adrenals. Neurosci Lett, 1989. 101(1): p. 101-6.

78. Wu, C.Y., et al., L-type calcium channels in sympathetic alpha3beta2-nAChRmediated cerebral nitrergic neurogenic vasodilation. Acta Physiol (Oxf), 2014. 211(4): p. 544-58.

79. Liu, Y., et al., Cav1.2, but not Cav1.3, L-type calcium channel subtype mediates nicotine-induced conditioned place preference in miceo. Prog Neuropsychopharmacol Biol Psychiatry, 2017. 75: p. 176-182.

80. Pappano, A.J. and R.A. Rembish, Nicotine-induced restoration of action potentials to cardiac tissue depolarized by potassium. Life Sci I, 1970. 9(24): p. 1381-8.

81. Satoh, H., Effects of nicotine on spontaneous activity and underlying ionic currents in rabbit sinoatrial nodal cells. Gen Pharmacol, 1997. 28(1): p. 39-44.

82. Carryl, O.R., A. Gallardo-Carpentier, and R.G. Carpentier, Effects of nicotine and ethanol on rat atrial membrane potentials. Alcohol, 1992. 9(2): p. 87-92.

83. Greenspan, K., et al., Some effects of nicotine on cardiac automaticity, conduction, and inotropy. Arch Intern Med, 1969. 123(6): p. 707-12.

84. Wang, H., H. Shi, and Z. Wang, Nicotine depresses the functions of multiple cardiac potassium channels. Life Sci, 1999. 65(12): p. PL143-9.

85. Wang, H., et al., Nicotine is a potent blocker of the cardiac A-type $K(+)$ channels. Effects on cloned Kv4.3 channels and native transient outward current. Circulation, 2000. 102(10): p. 1165-71.

86. Wang, H., et al., Direct block of inward rectifier potassium channels by nicotine. Toxicol Appl Pharmacol, 2000. 164(1): p. 97-101.

87. Brasch, H., H.B. Iven, and G. Zetler, Effects of simple indoles, noradrenaline, nicotine, and tyramine on action potential and contractility of the isolated guineapig papillary muscle. Naunyn Schmiedebergs Arch Pharmacol, 1977. 299(3): p. 259-65.

88. Pappano, A.J., Biphasic effect of nicotine on action potential repolarization in electrically driven guinea-pig atria. J Pharmacol Exp Ther, 1970. 172(2): p. 25565. 
89. Bassett, A.L. and H. Gelband, Nicotine and the action potential of cat ventricle. J Pharmacol Exp Ther, 1974. 188(1): p. 157-65.

90. Grassi, G., et al., Mechanisms responsible for sympathetic activation by cigarette smoking in humans. Circulation, 1994. 90(1): p. 248-53.

91. Cryer, P.E., et al., Norepinephrine and epinephrine release and adrenergic mediation of smoking-associated hemodynamic and metabolic events. N Engl J Med, 1976. 295(11): p. 573-7.

92. Berlin, I. and R.M. Anthenelli, Monoamine oxidases and tobacco smoking. Int J Neuropsychopharmacol, 2001. 4(1): p. 33-42.

93. Haussmann, H.J., Use of hazard indices for a theoretical evaluation of cigarette smoke composition. Chem Res Toxicol, 2012. 25(4): p. 794-810.

94. Kurhanewicz, N., et al., TRPA1 mediates changes in heart rate variability and cardiac mechanical function in mice exposed to acrolein. Toxicol Appl Pharmacol, 2017. 324: p. 51-60.

95. Kurhanewicz, N., et al., TRPA1 mediates the cardiac effects of acrolein through parasympathetic dominance but also sympathetic modulation in mice. Toxicol Appl Pharmacol, 2018. 347: p. 104-114.

96. Clark, M.S., M.J. Rand, and S. Vanov, Comparison of pharmacological activity of nicotine and related alkaloids occurring in cigarette smoke. Arch Int Pharmacodyn Ther, 1965. 156(2): p. 363-79.

97. Carreon-Garciduenas, M., et al., Participation of voltage-gated sodium and calcium channels in the acute cardiac effects of toluene. Toxicol Mech Methods, 2018. 28(9): p. 670-677.

98. Gordon, C.J., et al., Cardiovascular effects of oral toluene exposure in the rat monitored by radiotelemetry. Neurotoxicol Teratol, 2007. 29(2): p. 228-35.

99. Condouris, G.A. and D.M. Havelin, Acetaldehyde and cardiac arrhythmias. Arch Int Pharmacodyn Ther, 1987. 285(1): p. 50-9.

100. Tani, T. and Y. Horiguchi, Effects of formaldehyde on cardiac function. Jpn J Pharmacol, 1990. 52(4): p. 563-72.

101. Morvai, V., et al., ECG changes in benzene, toluene and xylene poisoned rats. Acta Med Acad Sci Hung, 1976. 33(3): p. 275-86.

102. Dallas, M.L., et al., Carbon monoxide induces cardiac arrhythmia via induction of the late Na+ current. Am J Respir Crit Care Med, 2012. 186(7): p. 648-56.

103. Tillar, R., T.J. Shafer, and J.J. Woodward, Toluene inhibits voltage-sensitive calcium channels expressed in pheochromocytoma cells. Neurochem Int, 2002. 41(6): p. 391-7.

104. Gauthereau, M.Y., E.M. Salinas-Stefanon, and S.L. Cruz, A mutation in the local anaesthetic binding site abolishes toluene effects in sodium channels. Eur J Pharmacol, 2005. 528(1-3): p. 17-26. 
105. Cruz, S.L., et al., Inhibition of cardiac sodium currents by toluene exposure. Br J Pharmacol, 2003. 140(4): p. 653-60.

106. Cruz, S.L., M.T. Rivera-Garcia, and J.J. Woodward, Review of toluene action: clinical evidence, animal studies and molecular targets. J Drug Alcohol Res, 2014. 3.

107. Lee, J.C. and S.E. Downing, Negative inotropic effects of phenol on isolated cardiac muscle. Am J Pathol, 1981. 102(3): p. 367-72.

108. Scragg, J.L., et al., Carbon monoxide inhibits L-type Ca2+ channels via redox modulation of key cysteine residues by mitochondrial reactive oxygen species. $\mathrm{J}$ Biol Chem, 2008. 283(36): p. 24412-9.

109. Dallas, M.L., J.L. Scragg, and C. Peers, Inhibition of L-type Ca(2+) channels by carbon monoxide. Adv Exp Med Biol, 2009. 648: p. 89-95.

110. Shrager, P.G., A. Strickholm, and R.I. Macey, Chemical modification of crayfish axons by protein crosslinking aldehydes. J Cell Physiol, 1969. 74(1): p. 91-100.

111. Kohlhardt, M., Modifications of drug-induced cardiac slow inward current block by cooling, $p H$ variations, and formaldehyde in mammalian ventricular myocardium. J Cardiovasc Pharmacol, 1983. 5(6): p. 968-77.

112. Nonner, W., B.C. Spalding, and B. Hille, Low intracellular pH and chemical agents slow inactivation gating in sodium channels of muscle. Nature, 1980. 284(5754): p. 360-3.

113. Williams, E.S., M.J. Mirro, and J.C. Bailey, Electrophysiological effects of ethanol, acetaldehyde, and acetate on cardiac tissues from dog and guinea pig. Circ Res, 1980. 47(3): p. 473-8.

114. Chen, F.S., et al., Effects of acetaldehyde on action potentials and Ca2+ currents in single atrial myocytes from the bullfrog. Pharmacology, 2012. 90(3-4): p. 21622.

115. Martinez, J.L. and M. Penna, Influences of changes in calcium concentrations, cocaine and clonidine on the cardiac effect of acetaldehyde in rat isolated atria. Gen Pharmacol, 1997. 29(2): p. 281-4.

116. Ju, Y.K. and D.G. Allen, Cyanide inhibits the $\mathrm{Na}+/ \mathrm{Ca}+$ exchanger in isolated cardiac pacemaker cells of the cane toad. Pflugers Arch, 2005. 449(5): p. 442-8.

117. Elies, J., et al., Inhibition of the cardiac Na(+) channel Nav1.5 by carbon monoxide. J Biol Chem, 2014. 289(23): p. 16421-9.

118. Ferreira de Mattos, G., et al., Lead poisoning: acute exposure of the heart to lead ions promotes changes in cardiac function and Cav1.2 ion channels. Biophys Rev, 2017. 9(5): p. 807-825.

119. DiFrancesco, D., et al., Cadmium-induced blockade of the cardiac fast $\mathrm{Na}$ channels in calf Purkinje fibres. Proc R Soc Lond B Biol Sci, 1985. 223(1233): p. 475-84. 
120. Wang, T., et al., Particulate matter induces cardiac arrhythmias via dysregulation of carotid body sensitivity and cardiac sodium channels. Am J Respir Cell Mol Biol, 2012. 46(4): p. 524-31.

121. Horakova, Z., et al., Effect of ethanol and acetaldehyde at clinically relevant concentrations on atrial inward rectifier potassium current IK1: separate and combined effect. J Physiol Pharmacol, 2016. 67(3): p. 339-51.

122. Bebarova, M., et al., Acetaldehyde at clinically relevant concentrations inhibits inward rectifier potassium current I(K1) in rat ventricular myocytes. Physiol Res, 2015. 64(6): p. 939-43.

123. Kawahara, H., et al., Effects of ethanol and acetaldehyde on the contraction of cultured Ito cells. Alcohol Alcohol Suppl, 1993. 1B: p. 9-14.

124. Williams, J.W., et al., Effect of ethanol and acetaldehyde on the $(\mathrm{Na}++\mathrm{K}+)$ activated adenosine triphosphatase activity of cardiac plasma membranes. Biochem Pharmacol, 1975. 24(1): p. 27-32.

125. Yamakura, T., J.M. Lewohl, and R.A. Harris, Differential effects of general anesthetics on $G$ protein-coupled inwardly rectifying and other potassium channels. Anesthesiology, 2001. 95(1): p. 144-53.

126. Sanguinetti, M.C. and N.K. Jurkiewicz, Two components of cardiac delayed rectifier $K+$ current. Differential sensitivity to block by class III antiarrhythmic agents. J Gen Physiol, 1990. 96(1): p. 195-215.

127. Bhave, G., et al., Small-molecule modulators of inward rectifier $K+$ channels: recent advances and future possibilities. Future Med Chem, 2010. 2(5): p. 757-74.

128. Dallas, M.L., et al., Carbon monoxide protects against oxidant-induced apoptosis via inhibition of Kv2.1. FASEB J, 2011. 25(5): p. 1519-30.

129. Liang, S., et al., Carbon monoxide inhibits inward rectifier potassium channels in cardiomyocytes. Nat Commun, 2014. 5: p. 4676.

130. Ficker, E., et al., Mechanisms of arsenic-induced prolongation of cardiac repolarization. Mol Pharmacol, 2004. 66(1): p. 33-44.

131. Drolet, B., C. Simard, and D.M. Roden, Unusual effects of a QT-prolonging drug, arsenic trioxide, on cardiac potassium currents. Circulation, 2004. 109(1): p. 269.

132. Graff, D.W., et al., Metal particulate matter components affect gene expression and beat frequency of neonatal rat ventricular myocytes. Environ Health Perspect, 2004. 112(7): p. 792-8.

133. D'Alessandro, A., et al., Nicotine, cigarette smoking and cardiac arrhythmia: an overview. Eur J Prev Cardiol, 2012. 19(3): p. 297-305.

134. Zevin, S., et al., Cardiovascular effects of carbon monoxide and cigarette smoking. J Am Coll Cardiol, 2001. 38(6): p. 1633-8.

135. Mancia, G., et al., Smoking impairs baroreflex sensitivity in humans. Am J Physiol, 1997. 273(3 Pt 2): p. H1555-60. 
136. Arosio, E., et al., Effects of smoking on cardiopulmonary baroreceptor activation and peripheral vascular resistance. Eur J Clin Invest, 2006. 36(5): p. 320-5.

137. Marie Hawkeswood, J., The effects of a nicotine nasal spray on baroreflex sensitivity and mood states over three days of smoking cessation. 2001, Thesis (M. Sc.)--Queen's University, Kingston, Ontario, Canada.

138. Perez, C.M., M.S. Hazari, and A.K. Farraj, Role of autonomic reflex arcs in cardiovascular responses to air pollution exposure. Cardiovasc Toxicol, 2015. 15(1): p. 69-78.

139. Hazari, M.S., et al., A single exposure to acrolein desensitizes baroreflex responsiveness and increases cardiac arrhythmias in normotensive and hypertensive rats. Cardiovasc Toxicol, 2014. 14(1): p. 52-63.

140. Perez, C.M., et al., Acrolein inhalation alters arterial blood gases and triggers carotid body-mediated cardiovascular responses in hypertensive rats. Inhal Toxicol, 2015. 27(1): p. 54-63.

141. Argacha, J.F., et al., Nicotine increases chemoreflex sensitivity to hypoxia in nonsmokers. J Hypertens, 2008. 26(2): p. 284-94.

142. Najem, B., et al., Acute cardiovascular and sympathetic effects of nicotine replacement therapy. Hypertension, 2006. 47(6): p. 1162-7.

143. Yue, Z., et al., Role of TRP channels in the cardiovascular system. Am J Physiol Heart Circ Physiol, 2015. 308(3): p. H157-82.

144. Xing, J., J. Lu, and J. Li, TRPA1 mediates amplified sympathetic responsiveness to activation of metabolically sensitive muscle afferents in rats with femoral artery occlusion. Front Physiol, 2015. 6: p. 249.

145. Hayano, J., et al., Respiratory sinus arrhythmia. A phenomenon improving pulmonary gas exchange and circulatory efficiency. Circulation, 1996. 94(4): p. 842-7.

146. Lange, P., et al., Relationship of the type of tobacco and inhalation pattern to pulmonary and total mortality. Eur Respir J, 1992. 5(9): p. 1111-7.

147. Tanaka, T., et al., Acute effects of nicotine content in cigarettes on coronary flow velocity and coronary flow reserve in men. Am J Cardiol, 1998. 82(10): p. 12758, A9.

148. Gullu, H., et al., Light cigarette smoking impairs coronary microvascular functions as severely as smoking regular cigarettes. Heart, 2007. 93(10): p. 12747.

149. Klein, L.W., et al., Acute coronary hemodynamic response to cigarette smoking in patients with coronary artery disease. J Am Coll Cardiol, 1984. 3(4): p. 879-86.

150. Martin, J.L., et al., Acute coronary vasoconstrictive effects of cigarette smoking in coronary heart disease. Am J Cardiol, 1984. 54(1): p. 56-60.

151. Ciftci, O., et al., Acute effects of smoking light cigarettes on coronary microvascular functions. Clin Cardiol, 2009. 32(4): p. 210-4. 
152. Nelson, M.D., et al., Acute Effect of Hookah Smoking on the Human Coronary Microcirculation. Am J Cardiol, 2016. 117(11): p. 1747-54.

153. Stavros Stavrakis, B.J.S., Paul Garabelli, Sunny S. Po, Role of the Autonomic Nervous System in Atrial Fibrillation, in Cardiac Electrophysiology: From Cell to Bedside, J.J. Douglas P. Zipes, William G. Stevenson, Editor. 2018, ELSEVIER. p. 419-425.

154. Gordan, R., J.K. Gwathmey, and L.H. Xie, Autonomic and endocrine control of cardiovascular function. World J Cardiol, 2015. 7(4): p. 204-14.

155. Tweed, J.O., et al., The endocrine effects of nicotine and cigarette smoke. Trends Endocrinol Metab, 2012. 23(7): p. 334-42.

156. Kapoor, D. and T.H. Jones, Smoking and hormones in health and endocrine disorders. Eur J Endocrinol, 2005. 152(4): p. 491-9.

157. Smith, C.J. and T.H. Fischer, Particulate and vapor phase constituents of cigarette mainstream smoke and risk of myocardial infarction. Atherosclerosis, 2001. 158(2): p. 257-67.

158. Alberg, A., The influence of cigarette smoking on circulating concentrations of antioxidant micronutrients. Toxicology, 2002. 180(2): p. 121-37.

159. van der Vaart, H., et al., Acute effects of cigarette smoke on inflammation and oxidative stress: a review. Thorax, 2004. 59(8): p. 713-21.

160. Edirisinghe, I. and I. Rahman, Cigarette smoke-mediated oxidative stress, shear stress, and endothelial dysfunction: role of VEGFR2. Ann N Y Acad Sci, 2010. 1203: p. 66-72.

161. Larue, D. and G. Kato, Reduced number of alpha- and beta-adrenergic receptors in the myocardium of rats exposed to tobacco smoke. Eur J Pharmacol, 1981. 70(4): p. 519-29.

162. Kannel, W.B., et al., Heart rate and cardiovascular mortality: the Framingham Study. Am Heart J, 1987. 113(6): p. 1489-94.

163. Johansen, C.D., et al., Resting, night-time, and 24 h heart rate as markers of cardiovascular risk in middle-aged and elderly men and women with no apparent heart disease. Eur Heart J, 2013. 34(23): p. 1732-9.

164. Zhang, D., X. Shen, and X. Qi, Resting heart rate and all-cause and cardiovascular mortality in the general population: a meta-analysis. CMAJ, 2016. 188(3): p. E53-63.

165. Arnold, J.M., et al., Resting heart rate: a modifiable prognostic indicator of cardiovascular risk and outcomes? Can J Cardiol, 2008. 24 Suppl A: p. 3A-8A.

166. Heart rate variability: standards of measurement, physiological interpretation and clinical use. Task Force of the European Society of Cardiology and the North American Society of Pacing and Electrophysiology. Circulation, 1996. 93(5): p. 1043-65. 
167. Tsuji, H., et al., Impact of reduced heart rate variability on risk for cardiac events. The Framingham Heart Study. Circulation, 1996. 94(11): p. 2850-5.

168. Acampa, M., P.E. Lazzerini, and G. Martini, Atrial Cardiopathy and SympathoVagal Imbalance in Cryptogenic Stroke: Pathogenic Mechanisms and Effects on Electrocardiographic Markers. Front Neurol, 2018. 9: p. 469.

169. O'Neal, W.T., et al., The association between acute mental stress and abnormal left atrial electrophysiology. J Cardiovasc Electrophysiol, 2017. 28(10): p. 11511157.

170. Chen, G., et al., Electrophysiocardiogram: For the first time EPCG has been recorded on human body surface. Int J Cardiol, 2016. 222: p. 499-503.

171. Bagliani, G., et al., PR Interval and Junctional Zone. Card Electrophysiol Clin, 2017. 9(3): p. 411-433.

172. Thijs, V.N., et al., Predictors for atrial fibrillation detection after cryptogenic stroke: Results from CRYSTAL AF. Neurology, 2016. 86(3): p. 261-9.

173. Carrazco, C., et al., Prevalence and Risk Factors for Paroxysmal Atrial Fibrillation and Flutter Detection after Cryptogenic Ischemic Stroke. J Stroke Cerebrovasc Dis, 2018. 27(1): p. 203-209.

174. Montalvo, M., et al., PR Interval Prolongation and Cryptogenic Stroke: A Multicenter Retrospective Study. J Stroke Cerebrovasc Dis, 2017. 26(10): p. 24162420.

175. Salu, Y. and M.L. Marcus, Computer simulation of the precordial QRS complex: effects of simulated changes in ventricular wall thickness and volume. Am Heart J, 1976. 92(6): p. 758-66.

176. Kashani, A. and S.S. Barold, Significance of QRS complex duration in patients with heart failure. J Am Coll Cardiol, 2005. 46(12): p. 2183-92.

177. Peguero, J.G., et al., Electrocardiographic Criteria for the Diagnosis of Left Ventricular Hypertrophy. J Am Coll Cardiol, 2017. 69(13): p. 1694-1703.

178. Madias, J.E., Low QRS voltage and its causes. J Electrocardiol, 2008. 41(6): p. 498-500.

179. Madias, J.E., QRS Voltage Changes in Heart Failure: A 3-Compartment Mechanistic Model and its Implications. Indian Pacing Electrophysiol J, 2010. 10(10): p. 464-73.

180. Usoro, A.O., et al., Risk of mortality in individuals with low QRS voltage and free of cardiovascular disease. Am J Cardiol, 2014. 113(9): p. 1514-7.

181. Shenasa, M. and H. Shenasa, Hypertension, left ventricular hypertrophy, and sudden cardiac death. Int J Cardiol, 2017. 237: p. 60-63.

182. Desai, A.D., et al., Prognostic Significance of Quantitative QRS Duration. Am J Med, 2006. 119(7): p. 600-6.

183. Hosseini, S.M. and M. Jamshir, Valsalva Maneuver and Strain-Related ECG Changes. Res Cardiovasc Med, 2015. 4(4): p. e28136. 
184. Saltykova, M.M., [Basic mechanisms of $Q R S$ voltage changes on ECG of healthy subjects during the exercise test]. Fiziol Cheloveka, 2015. 41(1): p. 74-82.

185. Vikram Kumar Yeragani, S.A., K. P. Seema, Rahul Kumar, Manuel Tancer, QRS Amplitude of ECG in Normal Humans: Effects of Orthostatic Challenge on Linear and Nonlinear Measures of Beat-to-Beat Variability. Cardiovascular Engineering, September 2005. 5(3): p. 135-40.

186. Karjalainen, J., et al., Relation between QT intervals and heart rates from 40 to 120 beats/min in rest electrocardiograms of men and a simple method to adjust QT interval values. J Am Coll Cardiol, 1994. 23(7): p. 1547-53.

187. Abildskov, J.A., Adrenergic effects of the QT interval of the electrocardiogram. Am Heart J, 1976. 92(2): p. 210-6.

188. Milne, J.R., et al., Effect of intravenous propranolol on QT interval. A new method of assessment. Br Heart J, 1980. 43(1): p. 1-6.

189. Browne, K.F., et al., Influence of the autonomic nervous system on the $Q-T$ interval in man. Am J Cardiol, 1982. 50(5): p. 1099-103.

190. Bexton, R.S., H.O. Vallin, and A.J. Camm, Diurnal variation of the QT interval-influence of the autonomic nervous system. Br Heart J, 1986. 55(3): p. 253-8.

191. Cappato, R., et al., Sympathetic and vagal influences on rate-dependent changes of QT interval in healthy subjects. Am J Cardiol, 1991. 68(11): p. 1188-93.

192. van Lien, R., et al., Ambulatory measurement of the ECG T-wave amplitude. Psychophysiology, 2015. 52(2): p. 225-37.

193. Kanters, J.K., et al., T(peak)T(end) interval in long QT syndrome. J Electrocardiol, 2008. 41(6): p. 603-8.

194. Yagishita, D., et al., Sympathetic nerve stimulation, not circulating norepinephrine, modulates T-peak to T-end interval by increasing global dispersion of repolarization. Circ Arrhythm Electrophysiol, 2015. 8(1): p. 174-85.

195. Malik, M., et al., Is the Tpeak-Tend interval as a measure of repolarization heterogeneity dead or just seriously wounded? Heart Rhythm, 2019. 16(6): p. 952-953.

196. Srinivasan, N.T., et al., Differences in the upslope of the precordial body surface ECG T wave reflect right to left dispersion of repolarization in the intact human heart. Heart Rhythm, 2019. 16(6): p. 943-951.

197. Schwartz, P.J. and T. Weiss, T-wave amplitude as an index of cardiac sympathetic activity: a misleading concept. Psychophysiology, 1983. 20(6): p. 696-701.

198. Morady, F., et al., Electrophysiologic effects of epinephrine in humans. J Am Coll Cardiol, 1988. 11(6): p. 1235-44.

199. Weiss, R., et al., Cardiac electrophysiologic effects of norepinephrine in human beings. Am Heart J, 1998. 135(6 Pt 1): p. 945-51. 
200. Stern, S. and S. Eisenberg, The effect of propranolol (Inderal) on the electrocardiogram of normal subjects. Am Heart J, 1969. 77(2): p. 192-5.

201. Frankl, W.S. and L.A. Soloff, The hemodynamic effects of propranolol hydrochloride after smoking. Am J Med Sci, 1967. 254(5): p. 623-8.

202. Coffman, J.D., Effect of propranolol on blood pressure and skin blood flow during cigarette smoking. J Clin Pharmacol J New Drugs, 1969. 9(1): p. 39-44.

203. Brunel, P., et al., Acute changes in forearm haemodynamics produced by cigarette smoking in healthy normotensive non-smokers are not influenced by propranolol or pindolol. Eur J Clin Pharmacol, 1992. 42(2): p. 143-6.

204. Writing Committee, M., et al., 2017 ACC/AHA/HRS guideline for the evaluation and management of patients with syncope: A report of the American College of Cardiology/American Heart Association Task Force on Clinical Practice Guidelines and the Heart Rhythm Society. Heart Rhythm, 2017. 14(8): p. e155e217.

205. Shimizu, W., et al., Diagnostic value of epinephrine test for genotyping LQT1, LQT2, and LQT3 forms of congenital long QT syndrome. Heart Rhythm, 2004. 1(3): p. 276-83.

206. Feely, J., J. Crooks, and I.H. Stevenson, The influence of age, smoking and hyperthyroidism on plasma propranolol steady state concentration. Br J Clin Pharmacol, 1981. 12(1): p. 73-8.

207. Lee, M.S., S. Magari, and D.C. Christiani, Cardiac autonomic dysfunction from occupational exposure to polycyclic aromatic hydrocarbons. Occup Environ Med, 2011. 68(7): p. 474-8.

208. Murata, K., et al., Changes in autonomic function as determined by ECG R-R interval variability in sandal, shoe and leather workers exposed to n-hexane, xylene and toluene. Neurotoxicology, 1994. 15(4): p. 867-75.

209. Mordukhovich, I., et al., Association Between Particulate Air Pollution and QT Interval Duration in an Elderly Cohort. Epidemiology, 2016. 27(2): p. 284-90.

210. Devlin, R.B., et al., Controlled exposure of humans with metabolic syndrome to concentrated ultrafine ambient particulate matter causes cardiovascular effects. Toxicol Sci, 2014. 140(1): p. 61-72.

211. Yusuf Karavelioglu, H.K., Macit Kalçık, Zekeriya Küçükdurmaz, Sultan Özkurt, Taner Sarak, Short-term effects of varenicline therapy on homogeneity of heart rate, atrioventricular conduction and ventricular repolarization. Curr Res Cardiol 2015. 2(2): p. 69 - 72.

212. Sharma, N.K., et al., ECG Changes in Young Healthy Smokers: A Simple and Cost-Effective Method to Assess Cardiovascular Risk According to Pack-Years of Smoking. J Assoc Physicians India, 2017. 65(6): p. 26-30.

213. Swathi K, S.G., Study of ECG Effects in Smokers and Normals. Journal of Pharmaceutical Sciences and Research, 2015. 7(3): p. 163 - 165. 
214. Metta Sandhya, U.S., Deverashetty Joyarani, Study of ECG changes and left ventricular diastolic dysfunction as hemodynamic markers of myocardial stress in chronic smokers. International Journal of Research in Medical Sciences, 2015. 3(3): p. $588-92$.

215. Renuka Devi, T.A., P. Sai Kumar, ECG Changes in Smokers and Non Smokers-A Comparative Study. Journal of Clinical and Diagnostic Research, 2013. 7(5): p. 824-826. .

216. Ilgenli, T.F., et al., The Effects of Cigarette Smoking on the Tp-e Interval, Tpe/QT Ratio and Tp-e/QTc Ratio. Adv Clin Exp Med, 2015. 24(6): p. 973-8.

217. Tasolar, H., et al., Effect of smoking on Tp-e interval, Tp-e/QT and Tp-e/QTc ratios as indices of ventricular arrhythmogenesis. Heart Lung Circ, 2014. 23(9): p. 827-32.

218. Ari, H., et al., The effect of varenicline on Tp-e interval, Tp-e/QT ratio and Tpe/QTc ratio in healthy smokers and nonsmokers. Cardiol J, 2015. 22(5): p. 551-6.

219. Zhang, Y., et al., Coffee, alcohol, smoking, physical activity and QT interval duration: results from the Third National Health and Nutrition Examination Survey. PLoS One, 2011. 6(2): p. e17584.

220. Goldenberg, I., et al., Cigarette smoking and the risk of supraventricular and ventricular tachyarrhythmias in high-risk cardiac patients with implantable cardioverter defibrillators. J Cardiovasc Electrophysiol, 2006. 17(9): p. 931-6.

221. Chatterjee, S., et al., Chronic effect of smoking on the electrocardiogram. Jpn Heart J, 1989. 30(6): p. 827-39.

222. Baden, L., et al., Smoking status and the electrocardiogram: a cross-sectional and longitudinal study. Arch Environ Health, 1982. 37(6): p. 365-9.

223. Khan IS, R.M., Amin R, Study of ECG Changes in Apparently Healthy Adult Male Smokers. Dinajpur Med Col J, 2011. 4(1): p. 7-14.

224. Hekim Karapinar, O.E., Mustafa Bulut, Selçuk Pala, Mustafa Akçakoyun,Ramazan Kargin, Irfan Barutcu, Ali Metin Esen, Effect of Habitual Cigarette Smoking on P Wave Duration and Dispersion. Kofluyolu Kalp Dergisi, 2010. 13(3): p. 10-13.

225. Goette, A., et al., Cigarette smoking induces atrial fibrosis in humans via nicotine. Heart, 2007. 93(9): p. 1056-63.

226. Salman S Siddiqui, S.N.H., Tanu Aggarwal, Deepankar, A comparison of 12 lead ECG status on tobacco smokers, tobacco chewers and nontobacco users. National Journal od Medical Research, 2013. 3(3): p. 203-5.

227. Venkatesh G and S. RM, A Study of Electrocardiographic changes in smokers compared to normal human beings. Biomedcal Research, 2010. 21(4): p. 389 392.

228. Karjalainen, J., et al., QT interval as a cardiac risk factor in a middle aged population. Heart, 1997. 77(6): p. 543-8. 
229. Ileri, M., et al., Effect of habitual smoking on QT interval duration and dispersion. Am J Cardiol, 2001. 88(3): p. 322-5.

230. Ozyurt, A., et al., Effects of Acute Carbon Monoxide Poisoning on ECG and Echocardiographic Parameters in Children. Cardiovasc Toxicol, 2017. 17(3): p. 326-334.

231. Vural, C., et al., Evaluation of cardiac autonomic function using heart rate variability in children with acute carbon monoxide poisoning. Cardiol Young, 2017. 27(9): p. 1662-1669.

232. Eroglu, M., et al., Carbon monoxide poisoning increases Tpeak-Tend dispersion and QTc dispersion. Cardiovasc J Afr, 2014. 25(3): p. 106-9.

233. Satran, D., et al., Cardiovascular manifestations of moderate to severe carbon monoxide poisoning. J Am Coll Cardiol, 2005. 45(9): p. 1513-6.

234. Kaya, H., et al., COHgb levels predict the long-term development of acute myocardial infarction in CO poisoning. Am J Emerg Med, 2016. 34(5): p. 840-4.

235. Volosin, K.J., et al., Effect of cigarette smoke on sinus node automaticity. Am J Cardiol, 1990. 65(3): p. 243-5.

236. Ondrejka, J. and G. Giorgio, Type 1 Brugada Pattern Associated with Nicotine Toxicity. J Emerg Med, 2015. 49(6): p. e183-6.

237. Mohammad Ali Akbarzadeh, S.Y., Mohamad Esmail Ghaidari, Mohammad Asadpour-Piranfar, Negar Bahrololoumi-Bafruee, Allahyar Golabchi, Amirhossein Azhari, Acute effects of smoking on QT dispersion in healthy males. ARYA Atheroscler, 2014. 10(2): p. 89 - 93.

238. Ramakrishnan, S., et al., Acute electrocardiographic changes during smoking: an observational study. BMJ Open, 2013. 3(4).

239. Sperry, R.E., J.L. Vacek, and G.S. Smith, The effects of smoking on the signalaveraged electrocardiogram in normal subjects. Chest, 1991. 99(1): p. 121-2.

240. Bekheit, S. and E. Fletcher, The effects of smoking on myocardial conduction in the human heart. Am Heart J, 1976. 91(6): p. 712-20.

241. Dinas, P.C., Y. Koutedakis, and A.D. Flouris, Effects of active and passive tobacco cigarette smoking on heart rate variability. Int J Cardiol, 2013. 163(2): p. 109-15.

242. Stead, L.F., et al., Nicotine replacement therapy for smoking cessation. Cochrane Database Syst Rev, 2008(1): p. CD000146.

243. Survey, N.H.a.N.E. 2009-2010 Data Documentation, Codebook, and Frequencies. September 2011; Cotinine - Serum \& Total NNAL - Urine (COTNAL_F) [Available from: https://wwwn.cdc.gov/nchs/nhanes/20092010/COTNAL_F.htm\#References.

244. Health, U.D.o. and H. Services, Third national health and nutrition examination survey, 1988-1994. NHANES III laboratory data file. Hyattsville, MD: Centers for Disease Control and Prevention, 1996. 
245. Benowitz, N.L., J. Hukkanen, and P. Jacob, 3rd, Nicotine chemistry, metabolism, kinetics and biomarkers. Handb Exp Pharmacol, 2009(192): p. 29-60.

246. Sagie, A., et al., An improved method for adjusting the QT interval for heart rate (the Framingham Heart Study). Am J Cardiol, 1992. 70(7): p. 797-801.

247. Soliman, E.Z., et al., Reference ranges of $P R$ duration and $P$-wave indices in individuals free of cardiovascular disease: the Multi-Ethnic Study of Atherosclerosis (MESA). J Electrocardiol, 2013. 46(6): p. 702-6.

248. Mason, J.W., et al., Electrocardiographic reference ranges derived from 79,743 ambulatory subjects. J Electrocardiol, 2007. 40(3): p. 228-34.

249. Santini, M., et al., Electrocardiographic characteristics, anthropometric features, and cardiovascular risk factors in a large cohort of adolescents. Europace, 2018. 20(11): p. 1833-1840.

250. Nelson, M.R., et al., Associations between electrocardiographic interval durations and coronary artery calcium scores: the Diabetes Heart Study. Pacing Clin Electrophysiol, 2008. 31(3): p. 314-21.

251. Usama Boles, A.E., Wael Al Ghabra, Hoshiar Abdollah, Kevin A. Michael, Early changes on the electrocardiogram in hypertension ESC Council for Cardiology Practice 2015. 13( $\mathrm{N}^{\circ} 30$ - 08).

252. Vaughan Williams, E.M., A classification of antiarrhythmic actions reassessed after a decade of new drugs. J Clin Pharmacol, 1984. 24(4): p. 129-47.

253. Plan and operation of the Third National Health and Nutrition Examination Survey, 1988-94. Series 1: programs and collection procedures. Vital Health Stat 1, 1994(32): p. 1-407.

254. Amit Srivastava, et al., A Comparative Study of Electrocardiographic Changes between Non smokers and Smokers International Journal of Computer Science Engineering \& Technology, 2012. 2(5): p. 1231.

255. M. Syamala Devi, D.T.L., Nazia Farah, Study of ECG changes among smokers. Journal of Evolution of Medical and Dental Sciences, 2014. 3(49): p. 11744 11748.

256. Deepmala J.Yadav, J.U.Y., A.A.Joshi, Study of Effects of Smoking on Electrocardiography in Smokers Compared to Non-smokers. International Journal of Medical and Health Sciences, 2015. 4(2): p. 190 - 195.

257. Mallikarjuna V, P.G., Arunkumar S, Prashanth KS, , A study of ECG changes in healthy smokers. International Journal Of Basic Medical Sciences, 2017. 7(5).

258. Renu Yadav, Subodh Kumar Yadav, and A. Karn, Electrocardiographic changes in young smokers: a cross-sectional study. Medicine Science International Medical Journal, 2016. 5(4): p. 945-947.

259. Yaniel Castro-Torresa, R.C.-P., Luis Castañeda-Carsarvilla, Increased maximum $p$ wave duration in smoking patients with ST-elevation acute myocardial 
infarction and its relationship with inflammatory markers. Cor et Vasa, 2017. 59(3): p. e246-e250.

260. Irfan, A., et al., Effects of chronic smoking on atrioventricular conduction and catecholamine production. Manuscript submitted for publication.

261. Holmqvist, F., et al., Clinical outcome as a function of the PR-interval-there is virtue in moderation: data from the Duke Databank for cardiovascular disease. Europace, 2015. 17(6): p. 978-85.

262. Dilaveris, P., et al., The effects of cigarette smoking on the heterogeneity of ventricular repolarization. Am Heart J, 2001. 142(5): p. 833-7.

263. Fauchier, L., et al., Association between heart rate-corrected QT interval and coronary risk factors in 2,894 healthy subjects (the DESIR Study). Data from an Epidemiological Study on the Insulin Resistance syndrome. Am J Cardiol, 2000. 86(5): p. 557-9, A9.

264. Thomakos, P., et al., Cigarette Smoking Is Associated with Prolongation of the QTc Interval Duration in Patients with Type 2 Diabetes Mellitus. Int J Endocrinol, 2013. 2013: p. 329189.

265. Romero Mestre, J.C., et al., [Studies of cardiovascular autonomic function and duration of QTc interval in smokers]. Rev Esp Cardiol, 1996. 49(4): p. 259-63.

266. Goldberger, A.L. and V. Bhargava, Effect of exercise on QRS duration in healthy men: a computer ECG analysis. J Appl Physiol Respir Environ Exerc Physiol, 1983. 54(4): p. 1083-8.

267. Frederiks, J., et al., Within-subject electrocardiographic differences at equal heart rates: role of the autonomic nervous system. Pflugers Arch, 2001. 441(5): p. 71724.

268. Kozek, E., et al., The relation between time- and frequency-domain measures of heart rate variability, QT interval and dispersion, JT interval and corrected JT in patients with type 1 diabetes. Practical Diabetology, 2006. 7(6): p. 382-389.

269. Conrath, C.E. and T. Opthof, Ventricular repolarization: an overview of (patho)physiology, sympathetic effects and genetic aspects. Prog Biophys Mol Biol, 2006. 92(3): p. 269-307.

270. Bebarova, M., Z. Horakova, and R. Kula, Addictive drugs, arrhythmias, and cardiac inward rectifiers. Europace, 2017. 19(3): p. 346-355.

271. Mizobe, F. and B.G. Livett, Nicotine stimulates secretion of both catecholamines and acetylcholinesterase from cultured adrenal chromaffin cells. J Neurosci, 1983. 3(4): p. 871-6.

272. Lundby, A., et al., In vivo phosphoproteomics analysis reveals the cardiac targets of beta-adrenergic receptor signaling. Sci Signal, 2013. 6(278): p. rs11.

273. Goette, A., Nicotine, atrial fibrosis, and atrial fibrillation: do microRNAs help to clear the smoke? Cardiovasc Res, 2009. 83(3): p. 421-2. 
274. Csonka, E., et al., The effect of nicotine on cultured cells of vascular origin. Virchows Arch A Pathol Anat Histopathol, 1985. 407(4): p. 441-7.

275. Zimmerman, M. and J. McGeachie, The effect of nicotine on aortic endothelium. A quantitative ultrastructural study. Atherosclerosis, 1987. 63(1): p. 33-41.

276. AP Carll, A.F., AM Roberts, The Role of the Autonomic Nervous System in Cardiovascular Toxicity, in Comprehensive Toxicology, C.A. McQueen, Editor. 2018, Elsevier. p. 61-114.

277. DeJarnett, N., et al., Acrolein exposure is associated with increased cardiovascular disease risk. J Am Heart Assoc, 2014. 3(4).

278. Goldberger, A.L., Electrocardiography in Harrison's Principles of Internal Medicine, K.D. F.A. Longo DL, Hauser SL, Jameons JL, Loscalzo J, Editor. 2012, New York: McGraw-Hill; 2012. .

279. Salles, G., et al., Prognostic value of QT interval parameters for mortality risk stratification in Chagas' disease: results of a long-term follow-up study. Circulation, 2003. 108(3): p. 305-12.

280. Man, C.N., et al., Simple, rapid and sensitive assay method for simultaneous quantification of urinary nicotine and cotinine using gas chromatography-mass spectrometry. J Chromatogr B Analyt Technol Biomed Life Sci, 2006. 844(2): p. 322-7.

281. Preacher, K.J. and A.F. Hayes, SPSS and SAS procedures for estimating indirect effects in simple mediation models. Behav Res Methods Instrum Comput, 2004. 36(4): p. 717-31.

282. Metta Sandhya, Uppala Satyanarayana, and D. Joyarani, Study of ECG changes and left ventricular diastolic dysfunction as hemodynamic markers of myocardial stress in chronic smokers. International Journal of Research in Medical Sciences, 2015. 3(3): p. 588 - 592.

283. Nielsen, J.B., et al., Risk of atrial fibrillation as a function of the electrocardiographic PR interval: results from the Copenhagen ECG Study. Heart Rhythm, 2013. 10(9): p. 1249-56.

284. Watanabe, I., Smoking and risk of atrial fibrillation. J Cardiol, 2018. 71(2): p. 111-112.

285. Hill, P. and E.L. Wynder, Smoking and cardiovascular disease. Effect of nicotine on the serum epinephrine and corticoids. Am Heart J, 1974. 87(4): p. 491-6.

286. Fowler, J.S., et al., Monoamine oxidase and cigarette smoking. Neurotoxicology, 2003. 24(1): p. 75-82.

287. Launay, J.M., et al., Smoking induces long-lasting effects through a monoamineoxidase epigenetic regulation. PLoS One, 2009. 4(11): p. e7959.

288. Brodde, O.E. and M.C. Michel, Adrenergic and muscarinic receptors in the human heart. Pharmacol Rev, 1999. 51(4): p. 651-90. 
289. Bianchi, C., et al., Effects of dobutamine on atrioventricular conduction. Am Heart J, 1975. 90(4): p. 474-8.

290. Nishikawa, K., et al., The effects of dobutamine and phenylephrine on atrioventricular conduction during combined use of halothane and thoracic epidural lidocaine. Anesth Analg, 1996. 82(3): p. 551-7.

291. Karolewicz, B., et al., Effects of depression, cigarette smoking, and age on monoamine oxidase B in amygdaloid nuclei. Brain Res, 2005. 1043(1-2): p. 5764.

292. Picciotto, M.R., D.H. Brunzell, and B.J. Caldarone, Effect of nicotine and nicotinic receptors on anxiety and depression. Neuroreport, 2002. 13(9): p. 1097106.

293. Snider, S.R. and O. Kuchel, Dopamine: an important neurohormone of the sympathoadrenal system. Significance of increased peripheral dopamine release for the human stress response and hypertension. Endocr Rev, 1983. 4(3): p. 291309.

294. Danielson, K., et al., The effects of nicotine and tobacco particulate matter on dopamine uptake in the rat brain. Synapse, 2014. 68(2): p. 45-60.

295. Motomura, S. and K. Hashimoto, Beta 2-adrenoceptor-mediated positive dromotropic effects on atrioventricular node of dogs. Am J Physiol, 1992. 262(1 Pt 2): p. H123-9.

296. Townshend, M.M. and A.J. Smith, Factors influencing the urinary excretion of free catecholamines in man. Clin Sci, 1973. 44(3): p. 253-65.

297. Dorr, M., et al., beta-blocker therapy and heart rate control during exercise testing in the general population: role of a common G-protein beta-3 subunit variant. Pharmacogenomics, 2010. 11(9): p. 1209-21.

298. Luceri, R.M., et al., PR interval behavior during exercise: implications for physiological pacemakers. Pacing Clin Electrophysiol, 1990. 13(12 Pt 2): p. 1719-23.

299. Carll, A.P., A.K. Farraj, and A.M. Roberts, The Role of the Autonomic Nervous System in Cardiovascular Toxicity, in Comprehensive Toxicology, M.J. Campen, Editor. 2018, Elsevier, Ltd.: Oxford.

300. Centers for Disease, C. and Prevention, Annual smoking-attributable mortality, years of potential life lost, and economic costs--United States, 1995-1999. MMWR Morb Mortal Wkly Rep, 2002. 51(14): p. 300-3.

301. Mortality, G.B.D. and C. Causes of Death, Global, regional, and national life expectancy, all-cause mortality, and cause-specific mortality for 249 causes of death, 1980-2015: a systematic analysis for the Global Burden of Disease Study 2015. Lancet, 2016. 388(10053): p. 1459-1544.

302. Grassi, G., G. Seravalle, and G. Mancia, Sympathetic activation in cardiovascular disease: evidence, clinical impact and therapeutic implications. Eur J Clin Invest, 2015. 45(12): p. 1367-75. 
303. Malpas, S.C., Sympathetic nervous system overactivity and its role in the development of cardiovascular disease. Physiol Rev, 2010. 90(2): p. 513-57.

304. Goniewicz, M.L., et al., High exposure to nicotine among adolescents who use Juul and other vape pod systems ('pods'). Tob Control, 2018.

305. Shusterman, V. and R. Lampert, Role of Stress in Cardiac Arrhythmias. J Atr Fibrillation, 2013. 5(6): p. 834.

306. Anderson, E.A., C.A. Sinkey, and A.L. Mark, Mental stress increases sympathetic nerve activity during sustained baroreceptor stimulation in humans. Hypertension, 1991. 17(4 Suppl): p. III43-9.

307. Mancia, G., Short- and long-term blood pressure variability: present and future. Hypertension, 2012. 60(2): p. 512-7.

308. Willich, S.N., et al., Sudden cardiac death. Support for a role of triggering in causation. Circulation, 1993. 87(5): p. 1442-50.

309. Mittleman, M.A., et al., Triggering of acute myocardial infarction onset by episodes of anger. Determinants of Myocardial Infarction Onset Study Investigators. Circulation, 1995. 92(7): p. 1720-5.

310. Porthan, K., et al., Predictive value of electrocardiographic QT interval and Twave morphology parameters for all-cause and cardiovascular mortality in a general population sample. Heart Rhythm, 2009. 6(8): p. 1202-8, 1208 e1.

311. Alonso, A., et al., Simple risk model predicts incidence of atrial fibrillation in a racially and geographically diverse population: the CHARGE-AF consortium. J Am Heart Assoc, 2013. 2(2): p. e000102.

312. Nielsen, J.B., et al., $P$-wave duration and the risk of atrial fibrillation: Results from the Copenhagen ECG Study. Heart Rhythm, 2015. 12(9): p. 1887-95.

313. Nielsen, J.B., et al., J-shaped association between QTc interval duration and the risk of atrial fibrillation: results from the Copenhagen ECG study. J Am Coll Cardiol, 2013. 61(25): p. 2557-64.

314. Kraiczi, H., A. Hansson, and R. Perfekt, Single-dose pharmacokinetics of nicotine when given with a novel mouth spray for nicotine replacement therapy. Nicotine Tob Res, 2011. 13(12): p. 1176-82.

315. Picavet, P., et al., Comparison of the Pharmacokinetics of Nicotine Following Single and Ad Libitum Use of a Tobacco Heating System or Combustible Cigarettes. Nicotine Tob Res, 2016. 18(5): p. 557-63.

316. Muntner, P., et al., Measurement of Blood Pressure in Humans: A Scientific Statement From the American Heart Association. Hypertension, 2019. 73(5): p. e35-e66.

317. Kligfield, P., et al., Recommendations for the standardization and interpretation of the electrocardiogram: part I: The electrocardiogram and its technology: a scientific statement from the American Heart Association Electrocardiography and Arrhythmias Committee, Council on Clinical Cardiology; the American 
College of Cardiology Foundation; and the Heart Rhythm Society: endorsed by the International Society for Computerized Electrocardiology. Circulation, 2007. 115(10): p. 1306-24.

318. Tai, M.M., A mathematical model for the determination of total area under glucose tolerance and other metabolic curves. Diabetes Care, 1994. 17(2): p. 1524.

319. Bruce R.Hargreavesa, T.P.M., Polynomial Trendline function flaws in Microsoft Excel. Computational Statistics \& Data Analysis, 2010. 54(4): p. 1190 -1196.

320. Narkiewicz, K., et al., Cigarette smoking increases sympathetic outflow in humans. Circulation, 1998. 98(6): p. 528-34.

321. Moheimani, R.S., et al., Sympathomimetic Effects of Acute E-Cigarette Use: Role of Nicotine and Non-Nicotine Constituents. J Am Heart Assoc, 2017. 6(9).

322. Cooke, W.H., et al., Acute inhalation of vaporized nicotine increases arterial pressure in young non-smokers: a pilot study. Clin Auton Res, 2015. 25(4): p. 267-70.

323. Sjoberg, N. and D.A. Saint, A single $4 \mathrm{mg}$ dose of nicotine decreases heart rate variability in healthy nonsmokers: implications for smoking cessation programs. Nicotine Tob Res, 2011. 13(5): p. 369-72.

324. Shah, S.A., et al., Relation of short-term heart rate variability to incident heart failure (from the Multi-Ethnic Study of Atherosclerosis). Am J Cardiol, 2013. 112(4): p. 533-40.

325. La Rovere, M.T., et al., Short-term heart rate variability strongly predicts sudden cardiac death in chronic heart failure patients. Circulation, 2003. 107(4): p. 56570.

326. La Rovere, M.T., et al., Baroreflex sensitivity and heart rate variability in the identification of patients at risk for life-threatening arrhythmias: implications for clinical trials. Circulation, 2001. 103(16): p. 2072-7.

327. Antzelevitch, C. and A. Burashnikov, Overview of Basic Mechanisms of Cardiac Arrhythmia. Card Electrophysiol Clin, 2011. 3(1): p. 23-45.

328. Kasser, I. and J.W. Kennedy, The relationship of increased left atrial volume and pressure to abnormal $P$ waves on the electrocardiogram. Circulation, 1969. 39(3): p. 339-43.

329. Young, D.B., Control of cardiac output. Integrated systems physiology : from molecule to function ; lecture \#6. 2010, United States: San Rafael, CA : Morgan \& Claypool Life Sciences, c2010.

330. Kyriakides, Z.S., et al., Acute effects of cigarette smoking on left ventricular diastolic function. Eur Heart J, 1992. 13(6): p. 743-8.

331. Alam, M., et al., Acute effects of smoking on diastolic function in healthy participants: studies by conventional doppler echocardiography and doppler tissue imaging. J Am Soc Echocardiogr, 2002. 15(10 Pt 2): p. 1232-7. 
332. Chierchia, S., et al., Role of the sympathetic nervous system in the pathogenesis of chronic stable angina. Implications for the mechanism of action of beta-blockers. Circulation, 1990. 82(3 Suppl): p. II71-81.

333. Sundkvist, G.M., et al., Mechanisms of exercise-induced ST-segment depression in patients without typical angina pectoris. J Intern Med, 2007. 261(2): p. 148-58.

334. Furberg, C., Adrenergic beta-blockade and electrocardiographical ST-T changes. Acta Med Scand, 1967. 181(1): p. 21-32.

335. Ponikowski, P., et al., Transient autonomic dysfunction precedes ST-segment depression in patients with syndrome X. Am J Cardiol, 1996. 77(11): p. 942-7.

336. Boudoulas, H., et al., Effect of increased adrenergic activity on the relationship between electrical and mechanical systole. Circulation, 1981. 64(1): p. 28-33.

337. Dukes, I.D. and E.M. Vaughan Williams, Effects of selective alpha 1-, alpha 2-, beta 1-and beta 2-adrenoceptor stimulation on potentials and contractions in the rabbit heart. J Physiol, 1984. 355: p. 523-46.

338. Wannamethee, S.G., et al., Serum potassium, cigarette smoking, and mortality in middle-aged men. Am J Epidemiol, 1997. 145(7): p. 598-606.

339. Kardalas, E., et al., Hypokalemia: a clinical update. Endocr Connect, 2018. 7(4): p. R135-R146.

340. Lachman, N., et al., Correlative anatomy for the electrophysiologist, part II: cardiac ganglia, phrenic nerve, coronary venous system. J Cardiovasc Electrophysiol, 2011. 22(1): p. 104-10.

341. Singh, S., et al., Topography of cardiac ganglia in the adult human heart. $\mathbf{J}$ Thorac Cardiovasc Surg, 1996. 112(4): p. 943-53.

342. Goette, A., et al., EHRA/HRS/APHRS/SOLAECE expert consensus on atrial cardiomyopathies: Definition, characterization, and clinical implication. Heart Rhythm, 2017. 14(1): p. e3-e40.

343. Gembala, M.I., et al., Acute changes in left ventricular diastolic function: cigarette smoking versus nicotine gum. Clin Cardiol, 2006. 29(2): p. 61-4.

344. Benowitz, N.L., et al., Nicotine absorption and cardiovascular effects with smokeless tobacco use: comparison with cigarettes and nicotine gum. Clin Pharmacol Ther, 1988. 44(1): p. 23-8.

345. Digard, H., et al., Determination of nicotine absorption from multiple tobacco products and nicotine gum. Nicotine Tob Res, 2013. 15(1): p. 255-61.

346. Horio, T., Effects of various taste stimuli on heart rate in humans. Chem Senses, 2000. 25(2): p. 149-53.

347. Bhatnagar, A., Cardiovascular Perspective of the Promises and Perils of ECigarettes. Circ Res, 2016. 118(12): p. 1872-5.

348. Bhatnagar, A., et al., New and Emerging Tobacco Products and the Nicotine Endgame: The Role of Robust Regulation and Comprehensive Tobacco Control 
and Prevention: A Presidential Advisory From the American Heart Association. Circulation, 2019. 139(19): p. e937-e958.

349. Berry, K.M., et al., Association of Electronic Cigarette Use With Subsequent Initiation of Tobacco Cigarettes in US Youths. JAMA Netw Open, 2019. 2(2): p. e187794.

350. Cullen, K.A., et al., Notes from the Field: Use of Electronic Cigarettes and Any Tobacco Product Among Middle and High School Students - United States, 2011 2018. MMWR Morb Mortal Wkly Rep, 2018. 67(45): p. 1276-1277.

351. Wang, J.B., et al., Cigarette and e-cigarette dual use and risk of cardiopulmonary symptoms in the Health eHeart Study. PLoS One, 2018. 13(7): p. e0198681.

352. Bhatta, D.N. and S.A. Glantz, Electronic Cigarette Use and Myocardial Infarction Among Adults in the US Population Assessment of Tobacco and Health. J Am Heart Assoc, 2019. 8(12): p. e012317.

353. Fearon, I.M., et al., Nicotine pharmacokinetics of electronic cigarettes: A review of the literature. Regul Toxicol Pharmacol, 2018. 100: p. 25-34.

354. Ogunwale, M.A., et al., Aldehyde Detection in Electronic Cigarette Aerosols. ACS Omega, 2017. 2(3): p. 1207-1214.

355. Kosmider, L., et al., Carbonyl compounds in electronic cigarette vapors: effects of nicotine solvent and battery output voltage. Nicotine Tob Res, 2014. 16(10): p. 1319-26.

356. Farsalinos, K.E., V. Voudris, and K. Poulas, E-cigarettes generate high levels of aldehydes only in 'dry puff' conditions. Addiction, 2015. 110(8): p. 1352-6.

357. Wagener, T.L., et al., Have combustible cigarettes met their match? The nicotine delivery profiles and harmful constituent exposures of second-generation and third-generation electronic cigarette users. Tob Control, 2017. 26(e1): p. e23e28.

358. Dawkins, L.E., et al., Self-titration by experienced e-cigarette users: blood nicotine delivery and subjective effects. Psychopharmacology (Berl), 2016. 233(15-16): p. 2933-41.

359. ; Available from: https://www.journalnow.com/business/juul-ends-with-percentmarket-share/article 6f50f427-19ec-50be-8b0c-d3df18d08759.html.

360. Omaiye, E.E., et al., High-Nicotine Electronic Cigarette Products: Toxicity of JUUL Fluids and Aerosols Correlates Strongly with Nicotine and Some Flavor Chemical Concentrations. Chem Res Toxicol, 2019. 32(6): p. 1058-1069.

361. Moheimani, R.S., et al., Increased Cardiac Sympathetic Activity and Oxidative Stress in Habitual Electronic Cigarette Users: Implications for Cardiovascular Risk. JAMA Cardiol, 2017. 2(3): p. 278-284.

362. Von Ahn, B., The acute effect of tobacco-smoking and nicotine on the electrocardiogram especially during induced hypoxia; a clinical and experimental investigation. Acta Med Scand Suppl, 1954. 292: p. 1-112. 
363. Lakhanpal A, K.M., Sultania S., A study of blood pressure and electrocardiography changes among smokers and non smokers. International Journal of Contemporary Medical Research 2018. 5(1): p. 32-35.

364. Irfan, A., et al., Association between serum cotinine levels and electrocardiographic left atrial abnormality. Ann Noninvasive Electrocardiol, 2019. 24(1): p. e12586.

365. January, C.T., et al., 2014 AHA/ACC/HRS guideline for the management of patients with atrial fibrillation: a report of the American College of Cardiology/American Heart Association Task Force on Practice Guidelines and the Heart Rhythm Society. J Am Coll Cardiol, 2014. 64(21): p. e1-76.

366. Guichard, J.B. and S. Nattel, Atrial Cardiomyopathy: A Useful Notion in Cardiac Disease Management or a Passing Fad? J Am Coll Cardiol, 2017. 70(6): p. 756765.

367. Algra, A., et al., QT interval variables from 24 hour electrocardiography and the two year risk of sudden death. Br Heart J, 1993. 70(1): p. 43-8.

368. Nielsen, J.B., et al., Risk prediction of cardiovascular death based on the QTC interval: evaluating age and gender differences in a large primary care population. Eur Heart J, 2014. 35(20): p. 1335-44.

369. Schimpf, R., M. Borggrefe, and C. Wolpert, Clinical and molecular genetics of the short QT syndrome. Curr Opin Cardiol, 2008. 23(3): p. 192-8. 


\section{APPENDIX}

PAGE

Abbreviations 146

Supplementary Tables

2.1.1 The 5th and 95th percentiles of PR interval, P duration, PR segment, corrected QT interval (QTc), QRS durations and uncorrected JT interval ...... 146

2.1.2 Patient characteristics by PR interval short $\left(<5^{\text {th }}\right.$ percentile), reference $\left(5-95^{\text {th }}\right.$ percentile) and long $\left(>95^{\text {th }}\right.$ percentile) groups

2.1.3 Patient characteristics by $\mathrm{P}$ duration short $\left(<5^{\text {th }}\right.$ percentile), reference $\left(5-95^{\text {th }}\right.$ percentile) and long $\left(>95^{\text {th }}\right.$ percentile) groups

2.1.4 Patient characteristics by PR segment short $\left(<5^{\text {th }}\right.$ percentile), reference $\left(5-95^{\text {th }}\right.$ percentile) and long $\left(>95^{\text {th }}\right.$ percentile) groups

2.1.5 Patient characteristics by corrected QT short $\left(<5^{\text {th }}\right.$ percentile), reference $\left(5-95^{\text {th }}\right.$ percentile) and long $\left(>95^{\text {th }}\right.$ percentile) groups

2.1.6 Patient characteristics by QRS duration short $\left(<5^{\text {th }}\right.$ percentile), reference $\left(5-95^{\text {th }}\right.$ percentile) and long $\left(>95^{\text {th }}\right.$ percentile) groups

2.1.7 Patient characteristics by (uncorrected) JT interval short $\left(<5^{\text {th }}\right.$ percentile), reference $\left(5-95^{\text {th }}\right.$ percentile) and long $\left(>95^{\text {th }}\right.$ percentile) groups

2.1.8 Association between serum cotinine levels (dichotomous) and abnormal PR segment, QRS duration and JT interval vs the reference group among subgroups by age and sex.

2.2.1 Baseline characteristics by high and low PR interval, $\mathrm{P}$ wave duration and PR segment

2.2.2 Distribution of urinary cotinine levels by self-reported active smoking status among all participants

2.2.3 Comparison among participants stratified by self-reported smoking status or by cotinine level of (A) urinary cotinine and catecholamines (median [interquartile range], creatinine-normalized), and (B) high and low PR interval, $\mathrm{P}$ wave duration and PR segment

2.2.4 Association between creatinine-normalized log transformed cotinine and parent catecholamines, their intermediate and final metabolites, and their metabolism, denoted by the ratios of intermediate: parent and final: intermediate metabolites 
2.2.5 Associations of QRS duration, QT interval and corrected QT with urinary cotinine in unadjusted and adjusted linear regressions

2.2.6 Association between catecholamine and atrial or atrioventricular conduction determined by unadjusted and adjusted linear regressions

3.1 Baseline characteristics 172

3.2 Correlation between Area under the Curve of root mean squared of successive differences (RMSSD) and rest of ECG waveform parameters

Supplementary Figures

2.2.1 Flowchart of selection of participants for study analysis

3.1 A) Flow chart to show the study design and enrollment B) Schematic Figureto illustrate the study visit procedures on different days 175

3.2 ECG morphological variables measured from Lead II..................................... 176

3.3 The Area Under the Curve for the baseline ECG parameters for each visit day among smokers

3.4 The raw values of several ECG parameters in pre-post and during exposure days among smokers

3.5 The raw $(\Delta)$ and relative $(\Delta \%)$ changes with standard errors of nicotine, Cotinine and 3-hyroycotinine (3-OH) levels from baseline per study visit ..... 181

3.6 The relationship between Area Under the Curves (AUC) of ECG parameters (Heart Rate, PR segment, ST height and root mean squared of successive differences (RMSSD)), with changes in Nicotine and Baseline Cotinine levels

3.7 The changes in Blood Pressure post cigarette and Nicotine Spray use (A) and their correlation with change in nicotine levels (B).... 183

Supplement text

Appendix A 155

Appendix B 184

Appendix C 186

Appendix D 189 


\section{LIST OF ABBREVIATIONS}

Cardiovascular disease (CVD)

Gamma-Aminobutyric Acid (GABA)

Electronic Nicotine Delivery Systems (ENDS)

Volatile Organic Compounds (VOCs)

Electrocardiogram (ECG)

Heart Rate Variability (HRV)

T peak - T end (Tp-Te)

Food and Drug Administration (FDA)

Autonomic Nervous System (ANS)

Sympathetic Nervous System (SNS)

Parasympathetic Nervous System (PNS)

Cyclic Adenosine monophosphate (cAMP)

Protein Kinase A (PKA)

G-protein-coupled inward rectifier potassium (GIRK)

Nicotinic Acetylcholinergic Receptors (nAchRs)

Third National Health and Nutrition Examination Survey (NHANES)

QT corrected (QTc)

Chronic Obstructive Pulmonary Disease (COPD)

Transient-Receptor Potential Ankyrin-1 channel (TRPA1)

Long QT syndrome (LQTS)

Fine Particulate Matter $\left(\mathrm{PM}_{2.5}\right)$

Monoamine oxidase (MOA)

Root of the Mean of Squared differences of Successive NN intervals (RMSSD) 
Supplementary Table 2.1.1 The 5th and 95th percentiles of PR interval, P duration, PR segment, corrected QT interval (QTc), QRS durations and uncorrected JT interval

\begin{tabular}{lcc}
\hline & $5^{\text {th }}$ percentile & $\begin{array}{c}95^{\text {th }} \\
\text { percentile }\end{array}$ \\
\hline PR interval (ms) & 126 & 209 \\
P duration (ms) & 92 & 134 \\
PR segment (ms) & 22 & 90 \\
QTc interval (ms) & 359 & 458 \\
QRS duration (ms) & 80 & 112 \\
JT interval (ms) & 264 & 361 \\
\hline
\end{tabular}


Supplementary Table 2.1.2 Patient characteristics by PR interval short ( $<5^{\text {th }}$ percentile), reference $\left(5-95^{\text {th }}\right.$ percentile $)$ and long $\left(>95^{\text {th }}\right.$ percentile $)$ groups

\begin{tabular}{|c|c|c|c|c|}
\hline \multirow{2}{*}{ Characteristic } & \multicolumn{3}{|c|}{ PR interval } & \multirow{2}{*}{ p-value } \\
\hline & Short & Reference & Long & \\
\hline Age (years) & $53.3 \pm 1.0$ & $55.3 \pm 0.4$ & $63.4 \pm 1.3$ & $<0.001$ \\
\hline Women & $177(66.9 \%)$ & $2698(54.4 \%)$ & $125(48.1 \%)$ & 0.025 \\
\hline Non-Hispanic White & $136(84.0 \%)$ & $2557(81.6 \%)$ & $167(83.8 \%)$ & 0.467 \\
\hline \multicolumn{5}{|l|}{ Smoking status } \\
\hline Never & $114(41.5 \%)$ & $2273(42.0 \%)$ & $128(46.2 \%)$ & \multirow{3}{*}{0.051} \\
\hline Current & $83(29.6 \%)$ & $1172(23.0 \%)$ & $42(12.9 \%)$ & \\
\hline Past & $72(29.0 \%)$ & $1665(35.0 \%)$ & $104(40.9 \%)$ & \\
\hline Diabetes mellitus & $21(7.2 \%)$ & $568(7.3 \%)$ & $33(7.2 \%)$ & 0.986 \\
\hline Hypertension & $73(21.1 \%)$ & $1714(31.0 \%)$ & $129(42.7 \%)$ & $<0.001$ \\
\hline Dyslipidemia & $63(23.9 \%)$ & $1234(27.7 \%)$ & $77(30.5 \%)$ & 0.399 \\
\hline Obesity & $38(12.1 \%)$ & $999(17.7 \%)$ & $49(14.3 \%)$ & 0.110 \\
\hline COPD & $27(10.4 \%)$ & $377(7.8 \%)$ & $19(7.9 \%)$ & 0.590 \\
\hline Heart rate (beats/minute) & $68.9 \pm 0.9$ & $68.1 \pm 0.3$ & $63.4 \pm 1.3$ & $<0.001$ \\
\hline Prior cardiovascular disease & $5(2.5 \%)$ & $202(3.0 \%)$ & $32(10.6 \%)$ & $<0.001$ \\
\hline Congestive Heart Failure & $5(1.0 \%)$ & $159(1.6 \%)$ & $21(5.9 \%)$ & 0.023 \\
\hline \multicolumn{5}{|l|}{ Alcohol drinks per month } \\
\hline 0 & $151(48.0 \%)$ & $2869(49.1 \%)$ & $157(52.8 \%)$ & \multirow{4}{*}{0.690} \\
\hline $1-4$ & $44(18.5 \%)$ & $826(17.6 \%)$ & $53(19.5 \%)$ & \\
\hline $5-13$ & $33(12.0 \%)$ & $579(13.8 \%)$ & $28(14.7 \%)$ & \\
\hline$>13$ & $40(21.5 \%)$ & $824(19.6 \%)$ & $34(12.9 \%)$ & \\
\hline$\beta$-blockers & $7(2.4 \%)$ & $358(7.4 \%)$ & $42(15.8 \%)$ & $<0.001$ \\
\hline Calcium channel blockers & $15(4.6 \%)$ & $369(6.0 \%)$ & $52(18.5 \%)$ & $<0.001$ \\
\hline Antiarrhythmic drugs & $2(1.6 \%)$ & $45(0.8 \%)$ & $4(1.4 \%)$ & 0.591 \\
\hline Cotinine $>15 \mathrm{ng} / \mathrm{ml}$ & $93(36.9 \%)$ & $1422(27.6 \%)$ & $65(19.9 \%)$ & 0.026 \\
\hline
\end{tabular}


Supplementary Table 2.1.3 Patient characteristics by $\mathrm{P}$ duration short $\left(<5^{\text {th }}\right.$ percentile), reference $\left(5-95^{\text {th }}\right.$ percentile $)$ and long $\left(>95^{\text {th }}\right.$ percentile $)$ groups

\begin{tabular}{|c|c|c|c|c|}
\hline \multirow{2}{*}{ Characteristic } & \multicolumn{3}{|c|}{$\mathrm{P}$ duration } & \multirow{2}{*}{ p-value } \\
\hline & Short & Reference & Long & \\
\hline Age (years) & $52.5 \pm 0.9$ & $55.5 \pm 0.4$ & $61.8 \pm 1.2$ & $<0.001$ \\
\hline Women & $187(67.1 \%)$ & $2714(54.5 \%)$ & $99(43.0 \%)$ & $<0.001$ \\
\hline Non-Hispanic White & $173(85.9 \%)$ & $2547(81.5 \%)$ & $140(82.3 \%)$ & 0.257 \\
\hline \multicolumn{5}{|l|}{ Smoking status } \\
\hline Never & $127(43.2 \%)$ & $2275(42.1 \%)$ & $113(41.1 \%)$ & \multirow{3}{*}{0.684} \\
\hline Current & $67(22.2 \%)$ & $1187(23.2 \%)$ & $43(17.9 \%)$ & \\
\hline Past & $83(34.6 \%)$ & $1663(34.7 \%)$ & $95(41.0 \%)$ & \\
\hline Diabetes mellitus & $23(5.2 \%)$ & $568(7.5 \%)$ & $31(7.2 \%)$ & 0.460 \\
\hline Hypertension & $68(20.7 \%)$ & $1732(31.1 \%)$ & $116(45.0 \%)$ & $<0.001$ \\
\hline Dyslipidemia & $60(21.6 \%)$ & $1255(27.9 \%)$ & $59(29.4 \%)$ & 0.127 \\
\hline Obesity & $38(11.5 \%)$ & $995(17.5 \%)$ & $53(20.9 \%)$ & 0.057 \\
\hline COPD & $22(6.6 \%)$ & $384(8.1 \%)$ & $17(5.4 \%)$ & 0.346 \\
\hline Heart rate (beats/minute) & $66.2 \pm 0.6$ & $68.2 \pm 0.3$ & $66.1 \pm 1.1$ & 0.009 \\
\hline Prior cardiovascular disease & $6(2.0 \%)$ & $209(3.3 \%)$ & $24(5.7 \%)$ & 0.114 \\
\hline Congestive Heart Failure & $3(0.2 \%)$ & $162(1.8 \%)$ & $20(3.9 \%)$ & 0.021 \\
\hline \multicolumn{5}{|l|}{ Alcohol drinks per month } \\
\hline 0 & $169(54.3 \%)$ & $2867(48.7 \%)$ & $141(52.5 \%)$ & \multirow{4}{*}{0.156} \\
\hline $1-4$ & $49(21.4 \%)$ & $838(17.7 \%)$ & $36(12.4 \%)$ & \\
\hline $5-13$ & $26(10.0 \%)$ & $586(14.0 \%)$ & $28(11.7 \%)$ & \\
\hline$>13$ & $33(14.2 \%)$ & $820(19.5 \%)$ & $45(23.4 \%)$ & \\
\hline$\beta$-blockers & $8(2.6 \%)$ & $361(7.5 \%)$ & $38(16.2 \%)$ & $<0.001$ \\
\hline Calcium channel blockers & $12(3.6 \%)$ & $388(6.4 \%)$ & $36(14.8 \%)$ & $<0.001$ \\
\hline Antiarrhythmic drugs & $3(1.5 \%)$ & $43(0.8 \%)$ & $5(1.9 \%)$ & 0.445 \\
\hline Cotinine $>15 \mathrm{ng} / \mathrm{ml}$ & $79(28.6 \%)$ & $1436(27.9 \%)$ & $65(24.2 \%)$ & 0.718 \\
\hline
\end{tabular}


Supplementary Table 2.1.4 Patient characteristics by PR segment short $\left(<5^{\text {th }}\right.$ percentile), reference $\left(5-95^{\text {th }}\right.$ percentile $)$ and long $\left(>95^{\text {th }}\right.$ percentile $)$ groups

\begin{tabular}{|c|c|c|c|c|}
\hline \multirow{2}{*}{ Characteristic } & \multicolumn{3}{|c|}{ PR segment } & \multirow{2}{*}{ p-value } \\
\hline & Short & Reference & Long & \\
\hline Age (years) & $55.6 \pm 1.0$ & $55.3 \pm 0.4$ & $60.2 \pm 1.4$ & 0.003 \\
\hline Women & $143(60.8 \%)$ & $2730(54.7 \%)$ & $127(51.2 \%)$ & 0.365 \\
\hline Non-Hispanic White & $120(82.2 \%)$ & $2575(81.7 \%)$ & $165(84.3 \%)$ & 0.626 \\
\hline \multicolumn{5}{|l|}{ Smoking status } \\
\hline Never & $99(30.9 \%)$ & $2299(42.8 \%)$ & $117(40.8 \%)$ & \multirow{3}{*}{0.010} \\
\hline Current & $94(35.6 \%)$ & $1149(22.5 \%)$ & $54(19.1 \%)$ & \\
\hline Past & $79(33.5 \%)$ & $1664(34.7 \%)$ & $98(40.1 \%)$ & \\
\hline Diabetes mellitus & $27(8.5 \%)$ & $563(7.3 \%)$ & $32(6.5 \%)$ & 0.770 \\
\hline Hypertension & $87(29.2 \%)$ & $1704(30.6 \%)$ & $125(40.5 \%)$ & 0.079 \\
\hline Dyslipidemia & $64(30.3 \%)$ & $1241(27.4 \%)$ & $69(28.9 \%)$ & 0.744 \\
\hline Obesity & $49(12.1 \%)$ & $989(17.7 \%)$ & $48(14.2 \%)$ & 0.139 \\
\hline COPD & $19(8.2 \%)$ & $384(7.8 \%)$ & $20(9.2 \%)$ & 0.823 \\
\hline Heart rate (beats/minute) & $70.5 \pm 0.8$ & $68.1 \pm 0.3$ & $63.6 \pm 0.9$ & $<0.001$ \\
\hline Prior cardiovascular disease & $6(1.9 \%)$ & $209(3.1 \%)$ & $24(7.4 \%)$ & 0.022 \\
\hline Congestive Heart Failure & $6(0.9 \%)$ & $160(1.7 \%)$ & $19(4.2 \%)$ & 0.050 \\
\hline \multicolumn{5}{|l|}{ Alcohol drinks per month } \\
\hline 0 & $140(39.3 \%)$ & $2883(49.7 \%)$ & $154(48.7 \%)$ & \multirow{4}{*}{0.690} \\
\hline $1-4$ & $39(20.3 \%)$ & $835(17.6 \%)$ & $49(19.4 \%)$ & \\
\hline $5-13$ & $38(15.6 \%)$ & $574(13.6 \%)$ & $28(13.5 \%)$ & \\
\hline$>13$ & $53(24.7 \%)$ & $809(19.2 \%)$ & $36(18.4 \%)$ & \\
\hline$\beta$-blockers & $15(3.6 \%)$ & $350(7.3 \%)$ & $42(15.8 \%)$ & 0.003 \\
\hline Calcium channel blockers & $14(4.2 \%)$ & $375(6.2 \%)$ & $47(15.5 \%)$ & 0.002 \\
\hline Antiarrhythmic drugs & $2(0.8 \%)$ & $47(0.9 \%)$ & $2(0.8 \%)$ & 0.999 \\
\hline Cotinine $>15 \mathrm{ng} / \mathrm{ml}$ & $105(41.2 \%)$ & $1399(27.3 \%)$ & $76(24.5 \%)$ & 0.009 \\
\hline
\end{tabular}


Supplementary Table 2.1.5 Patient characteristics by corrected QT short $\left(<5^{\text {th }}\right.$ percentile $)$, reference $\left(5-95^{\text {th }}\right.$ percentile $)$ and long $\left(>95^{\text {th }}\right.$ percentile $)$ groups

\begin{tabular}{|c|c|c|c|c|}
\hline \multirow{2}{*}{ Characteristic } & \multicolumn{3}{|c|}{ Corrected QT } & \multirow{2}{*}{ p-value } \\
\hline & Short & Reference & Long & \\
\hline Age (years) & $55.2 \pm 1.0$ & $55.4 \pm 0.4$ & $59.5 \pm 1.0$ & $<0.001$ \\
\hline Women & $109(49.9 \%)$ & $2725(54.8 \%)$ & $166(58.5 \%)$ & 0.506 \\
\hline Non-Hispanic White & $125(80.6 \%)$ & $2578(81.8 \%)$ & $157(83.3 \%)$ & 0.722 \\
\hline \multicolumn{5}{|l|}{ Smoking status } \\
\hline Never & $104(39.9 \%)$ & $2273(42.0 \%)$ & $138(46.7 \%)$ & \multirow{3}{*}{0.443} \\
\hline Current & $67(28.0 \%)$ & $1178(22.9 \%)$ & $52(18.6 \%)$ & \\
\hline Past & $91(32.1 \%)$ & $1658(35.1 \%)$ & $92(34.7 \%)$ & \\
\hline Diabetes mellitus & $45(11.3 \%)$ & $549(7.1 \%)$ & $28(9.0 \%)$ & 0.226 \\
\hline Hypertension & $102(31.7 \%)$ & $1687(30.5 \%)$ & $127(39.2 \%)$ & 0.131 \\
\hline Dyslipidemia & $67(28.9 \%)$ & $1244(27.7 \%)$ & $63(23.6 \%)$ & 0.473 \\
\hline Obesity & $50(20.1 \%)$ & $991(17.3 \%)$ & $45(14.0 \%)$ & 0.443 \\
\hline COPD & $35(16.0 \%)$ & $372(7.5 \%)$ & $16(9.5 \%)$ & 0.080 \\
\hline Heart rate (beats/minute) & $89.2 \pm 1.0$ & $67.9 \pm 0.2$ & $52.9 \pm 0.6$ & $<0.001$ \\
\hline Prior cardiovascular disease & $15(3.1 \%)$ & $206(3.2 \%)$ & $18(5.6 \%)$ & 0.236 \\
\hline Congestive Heart Failure & $15(3.1 \%)$ & $152(1.6 \%)$ & $18(3.1 \%)$ & 0.209 \\
\hline \multicolumn{5}{|l|}{ Alcohol drinks per month } \\
\hline 0 & $164(60.8 \%)$ & $2842(48.7 \%)$ & $171(47.6 \%)$ & \multirow{4}{*}{0.238} \\
\hline $1-4$ & $36(17.0 \%)$ & $841(18.0 \%)$ & $46(14.8 \%)$ & \\
\hline $5-13$ & $24(8.3 \%)$ & $586(13.6 \%)$ & $30(19.1 \%)$ & \\
\hline$>13$ & $37(13.9 \%)$ & $828(19.7 \%)$ & $33(18.6 \%)$ & \\
\hline$\beta$-blockers & $5(2.7 \%)$ & $341(7.1 \%)$ & $61(19.2 \%)$ & $<0.001$ \\
\hline Calcium channel blockers & $21(7.5 \%)$ & $371(6.1 \%)$ & $44(13.2 \%)$ & 0.005 \\
\hline Antiarrhythmic drugs & $3(1.0 \%)$ & $43(0.8 \%)$ & $5(1.5 \%)$ & 0.627 \\
\hline Cotinine $>15 \mathrm{ng} / \mathrm{ml}$ & $85(32.4 \%)$ & $1415(27.5 \%)$ & $80(28.4 \%)$ & 0.531 \\
\hline
\end{tabular}


Supplementary Table 2.1.6 Patient characteristics by QRS duration short $\left(<5^{\text {th }}\right.$ percentile $)$, reference $\left(5-95^{\text {th }}\right.$ percentile $)$ and long $\left(>95^{\text {th }}\right.$ percentile $)$ groups

\begin{tabular}{|c|c|c|c|c|}
\hline \multirow{2}{*}{ Characteristic } & \multicolumn{3}{|c|}{ QRS duration } & \multirow{2}{*}{ p-value } \\
\hline & Short & Reference & Long & \\
\hline Age (years) & $56.8 \pm 0.9$ & $55.6 \pm 0.4$ & $53.6 \pm 0.8$ & 0.003 \\
\hline Women & $204(80.3 \%)$ & $2726(55.1 \%)$ & $70(22.7 \%)$ & $<0.001$ \\
\hline Non-Hispanic White & $144(78.1 \%)$ & $2599(81.8 \%)$ & $117(86.1 \%)$ & 0.223 \\
\hline \multicolumn{5}{|l|}{ Smoking status } \\
\hline Never & $128(44.5 \%)$ & $2285(42.1 \%)$ & $102(41.3 \%)$ & \multirow{3}{*}{0.197} \\
\hline Current & $65(28.4 \%)$ & $1163(22.5 \%)$ & $69(25.3 \%)$ & \\
\hline Past & $72(27.0 \%)$ & $1680(35.4 \%)$ & $89(33.4 \%)$ & \\
\hline Diabetes mellitus & $46(11.5 \%)$ & $548(7.1 \%)$ & $28(7.3 \%)$ & 0.205 \\
\hline Hypertension & $91(34.1 \%)$ & $1730(30.9 \%)$ & $95(29.9 \%)$ & 0.704 \\
\hline Dyslipidemia & $72(30.7 \%)$ & $1248(27.5 \%)$ & $54(26.4 \%)$ & 0.684 \\
\hline Obesity & $44(12.2 \%)$ & $987(17.6 \%)$ & $55(15.9 \%)$ & 0.188 \\
\hline COPD & $29(10.6 \%)$ & $372(7.5 \%)$ & $22(13.6 \%)$ & 0.293 \\
\hline Heart rate (beats/minute) & $69.7 \pm 0.9$ & $68.0 \pm 0.2$ & $65.3 \pm 1.1$ & 0.003 \\
\hline Prior cardiovascular disease & $8(2.1 \%)$ & $220(3.3 \%)$ & $11(3.7 \%)$ & 0.566 \\
\hline Congestive Heart Failure & $14(1.3 \%)$ & $161(1.8 \%)$ & $10(2.1 \%)$ & 0.618 \\
\hline \multicolumn{5}{|l|}{ Alcohol drinks per month } \\
\hline 0 & $176(61.2 \%)$ & $2876(48.9 \%)$ & $125(41.2 \%)$ & \multirow{4}{*}{0.032} \\
\hline $1-4$ & $37(14.6 \%)$ & $841(17.9 \%)$ & $45(18.3 \%)$ & \\
\hline $5-13$ & $25(10.2 \%)$ & $583(14.0 \%)$ & $32(11.0 \%)$ & \\
\hline$>13$ & $27(14.0 \%)$ & $814(19.1 \%)$ & $57(29.5 \%)$ & \\
\hline$\beta$-blockers & $12(4.6 \%)$ & $376(7.6 \%)$ & $19(8.9 \%)$ & 0.301 \\
\hline Calcium channel blockers & $20(5.6 \%)$ & $396(6.5 \%)$ & $20(7.9 \%)$ & 0.584 \\
\hline Antiarrhythmic drugs & $1(0.1 \%)$ & $45(0.8 \%)$ & $5(2.2 \%)$ & 0.137 \\
\hline Cotinine $>15 \mathrm{ng} / \mathrm{ml}$ & $79(31.2 \%)$ & $1416(27.3 \%)$ & $85(33.0 \%)$ & 0.213 \\
\hline
\end{tabular}


Supplementary Table 2.1.7 Patient characteristics by (uncorrected) JT interval short $\left(<5^{\text {th }}\right.$ percentile), reference $\left(5-95^{\text {th }}\right.$ percentile $)$ and long $\left(>95^{\text {th }}\right.$ percentile $)$ groups

\begin{tabular}{|c|c|c|c|c|}
\hline \multirow{2}{*}{ Characteristic } & \multicolumn{3}{|c|}{ JT interval } & \multirow{2}{*}{ p-value } \\
\hline & Short & Reference & Long & \\
\hline Age (years) & $54.5 \pm 1.0$ & $55.3 \pm 0.4$ & $62.0 \pm 0.6$ & $<0.001$ \\
\hline Women & $90(34.5 \%)$ & $2745(55.5 \%)$ & $165(65.2 \%)$ & $<0.001$ \\
\hline Non-Hispanic White & $145(82.0 \%)$ & $2554(81.7 \%)$ & $161(83.5 \%)$ & 0.777 \\
\hline \multicolumn{5}{|l|}{ Smoking status } \\
\hline Never & $106(35.4 \%)$ & $2267(42.3 \%)$ & $142(46.5 \%)$ & \multirow{3}{*}{0.059} \\
\hline Current & $91(32.4 \%)$ & $1159(22.5 \%)$ & $47(19.2 \%)$ & \\
\hline Past & $107(32.2 \%)$ & $1653(35.1 \%)$ & $81(34.3 \%)$ & \\
\hline Diabetes mellitus & $52(11.6 \%)$ & $540(6.9 \%)$ & $30(10.3 \%)$ & 0.166 \\
\hline Hypertension & $118(34.2 \%)$ & $1679(30.3 \%)$ & $119(40.5 \%)$ & 0.049 \\
\hline Dyslipidemia & $74(27.4 \%)$ & $1242(27.7 \%)$ & $58(26.0 \%)$ & 0.913 \\
\hline Obesity & $64(22.6 \%)$ & $980(17.1 \%)$ & $42(14.6 \%)$ & 0.246 \\
\hline COPD & $32(12.2 \%)$ & $379(7.7 \%)$ & $12(6.2 \%)$ & 0.118 \\
\hline Heart rate (beats/minute) & $88.0 \pm 0.9$ & $67.5 \pm 0.2$ & $52.5 \pm 0.6$ & $<0.001$ \\
\hline Prior cardiovascular disease & $16(2.8 \%)$ & $206(3.1 \%)$ & $17(6.6 \%)$ & 0.107 \\
\hline Congestive Heart Failure & $17(2.1 \%)$ & $151(1.6 \%)$ & $17(3.4 \%)$ & 0.230 \\
\hline \multicolumn{5}{|l|}{ Alcohol drinks per month } \\
\hline 0 & $174(57.1 \%)$ & $2841(48.6 \%)$ & $162(51.8 \%)$ & \multirow{4}{*}{0.440} \\
\hline $1-4$ & $43(16.3 \%)$ & $832(17.8 \%)$ & $48(18.8 \%)$ & \\
\hline $5-13$ & $37(9.9 \%)$ & $582(14.1 \%)$ & $21(10.3 \%)$ & \\
\hline$>13$ & $49(16.7 \%)$ & $812(19.5 \%)$ & $37(19.1 \%)$ & \\
\hline B blockers & $7(2.6 \%)$ & $344(7.1 \%)$ & $56(21.3 \%)$ & $<0.001$ \\
\hline Calcium channel blockers & $20(5.4 \%)$ & $373(6.2 \%)$ & $43(14.1 \%)$ & 0.003 \\
\hline Antiarrhythmic drugs & $4(1.0 \%)$ & $44(0.9 \%)$ & $3(0.6 \%)$ & 0.883 \\
\hline Cotinine $>15 \mathrm{ng} / \mathrm{ml}$ & $110(37.3 \%)$ & $1391(27.1 \%)$ & $79(31.0 \%)$ & 0.039 \\
\hline
\end{tabular}


Supplementary Table 2.1.8 Association between serum cotinine levels (dichotomous) and abnormal PR segment, QRS duration and JT interval vs the reference group among subgroups by age and sex

\begin{tabular}{|c|c|c|c|c|c|c|c|}
\hline \multirow{2}{*}{\multicolumn{2}{|c|}{ Subgroups }} & \multicolumn{2}{|c|}{$\begin{array}{c}\text { PR segment } \\
<5^{\text {th }} \text { percentile vs reference }\end{array}$} & \multicolumn{2}{|c|}{$\begin{array}{c}\text { QRS duration } \\
<5^{\text {th }} \text { percentile vs reference }\end{array}$} & \multicolumn{2}{|c|}{$\begin{array}{c}\text { JT interval } \\
>95^{\text {th }} \text { percentile vs } \\
\text { reference }\end{array}$} \\
\hline & & $\begin{array}{l}\text { Odds ratio } \\
(95 \% \mathrm{CI})\end{array}$ & $\begin{array}{c}\text { Interaction } \\
\text { p-value }\end{array}$ & $\begin{array}{l}\text { Odds ratio } \\
(95 \% \mathrm{CI})\end{array}$ & $\begin{array}{l}\text { Interaction } \\
\text { p-value }\end{array}$ & $\begin{array}{l}\text { Odds ratio } \\
(95 \% \mathrm{CI})\end{array}$ & $\begin{array}{c}\text { Interaction } \\
\text { p-value }\end{array}$ \\
\hline \multirow[t]{2}{*}{ Age } & $\begin{array}{l}<59 \\
\text { years }\end{array}$ & $\begin{array}{c}1.92 \\
(1.08,3.42)\end{array}$ & \multirow{2}{*}{0.5661} & $\begin{array}{c}1.82 \\
(1.05,3.15)\end{array}$ & \multirow{2}{*}{0.3070} & $\begin{array}{c}1.69 \\
(0.80,3.58)\end{array}$ & \multirow{2}{*}{0.4007} \\
\hline & $\begin{array}{l}\geq 59 \\
\text { years }\end{array}$ & $\begin{array}{c}2.17 \\
(1.14,4.13)\end{array}$ & & $\begin{array}{c}1.22 \\
(0.76,1.95)\end{array}$ & & $\begin{array}{c}1.18 \\
(0.64,2.19)\end{array}$ & \\
\hline \multirow[t]{2}{*}{ Sex } & Male & $\begin{array}{c}2.26 \\
(1.13,4.50)\end{array}$ & \multirow{2}{*}{0.7550} & $\begin{array}{c}0.76 \\
(0.29,1.96)\end{array}$ & \multirow{2}{*}{0.0589} & $\begin{array}{c}1.49 \\
(0.72,3.11)\end{array}$ & \multirow{2}{*}{0.5171} \\
\hline & Female & $\begin{array}{c}1.82 \\
(1.03,3.20)\end{array}$ & & $\begin{array}{c}2.03 \\
(1.37,3.00)\end{array}$ & & $\begin{array}{c}1.74 \\
(0.92,3.31)\end{array}$ & \\
\hline
\end{tabular}




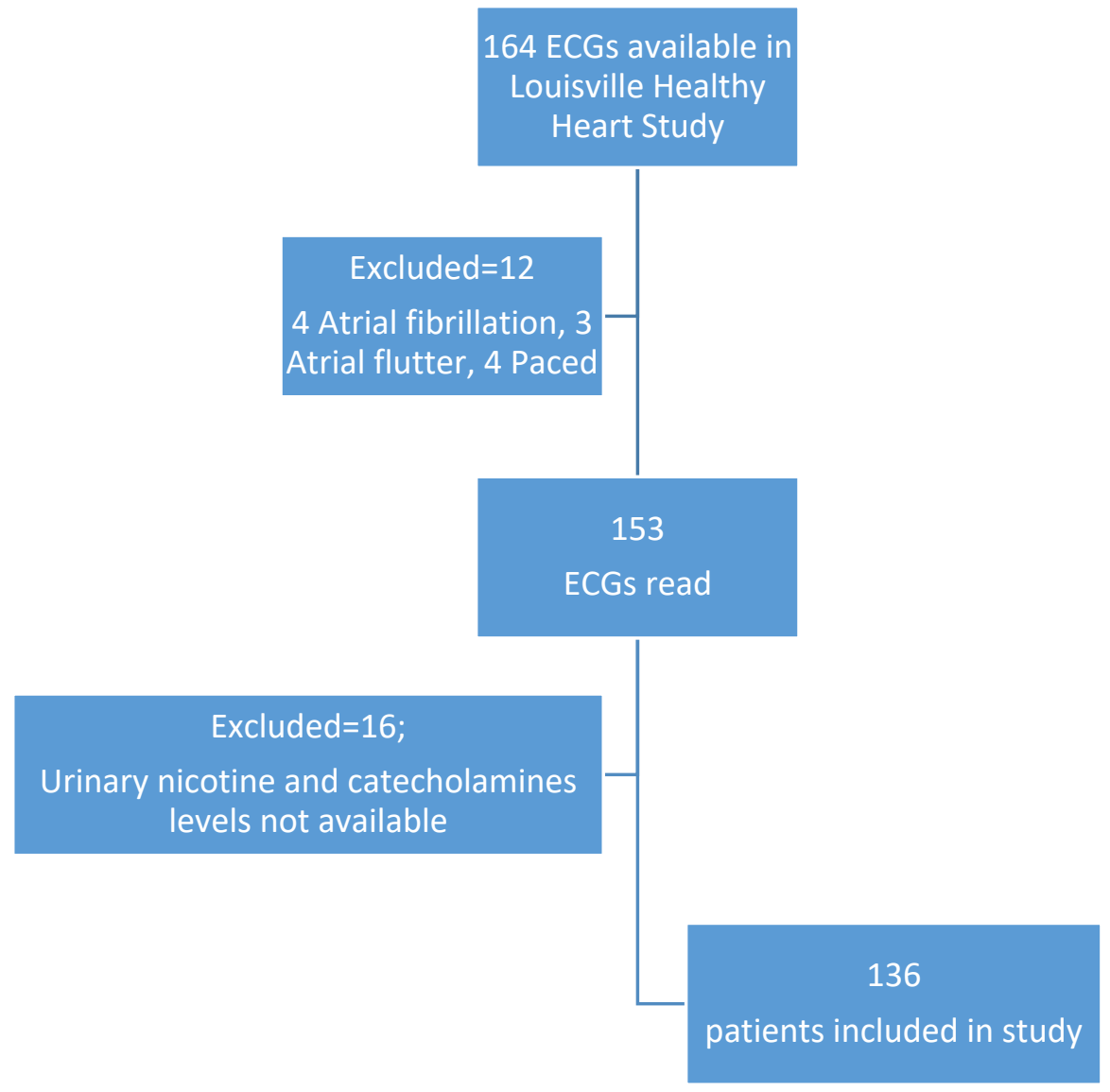

Supplementary Figure 2.2.1 Flowchart of selection of participants for study analysis 


\section{Appendix A}

ECG Measurement protocol for LHHS database

Exclude ECGs with Atrial fibrillation, Atrial flutter, paced rhythm.

The caliper measurements will be performed using electronic calipers and recorded up to 2 decimal points. Only waveforms in sinus rhythm will be included and measurements will be performed only on segments those are completely captured in that lead (ie complete QRS can be visualized in V6 and not QRS starting from V3 and transitioning into V6). Each segment will be measured and recorded in three consecutive waveforms. Incompletely recorded beats, premature atrial and ventricular beats will be excluded and the next complete sinus waveform in sequential order will be measured.

\section{$P$ wave duration}

$\mathrm{P}$ wave duration will be measured in lead II. Measurement will be conducted from the onset of the $\mathrm{P}$ wave, defined as the initial deflection from the isoelectric baseline of the TP segment, to the offset of the $\mathrm{P}$ wave, defined as the return of the $\mathrm{P}$ wave to the isoelectric baseline of the PR interval. 


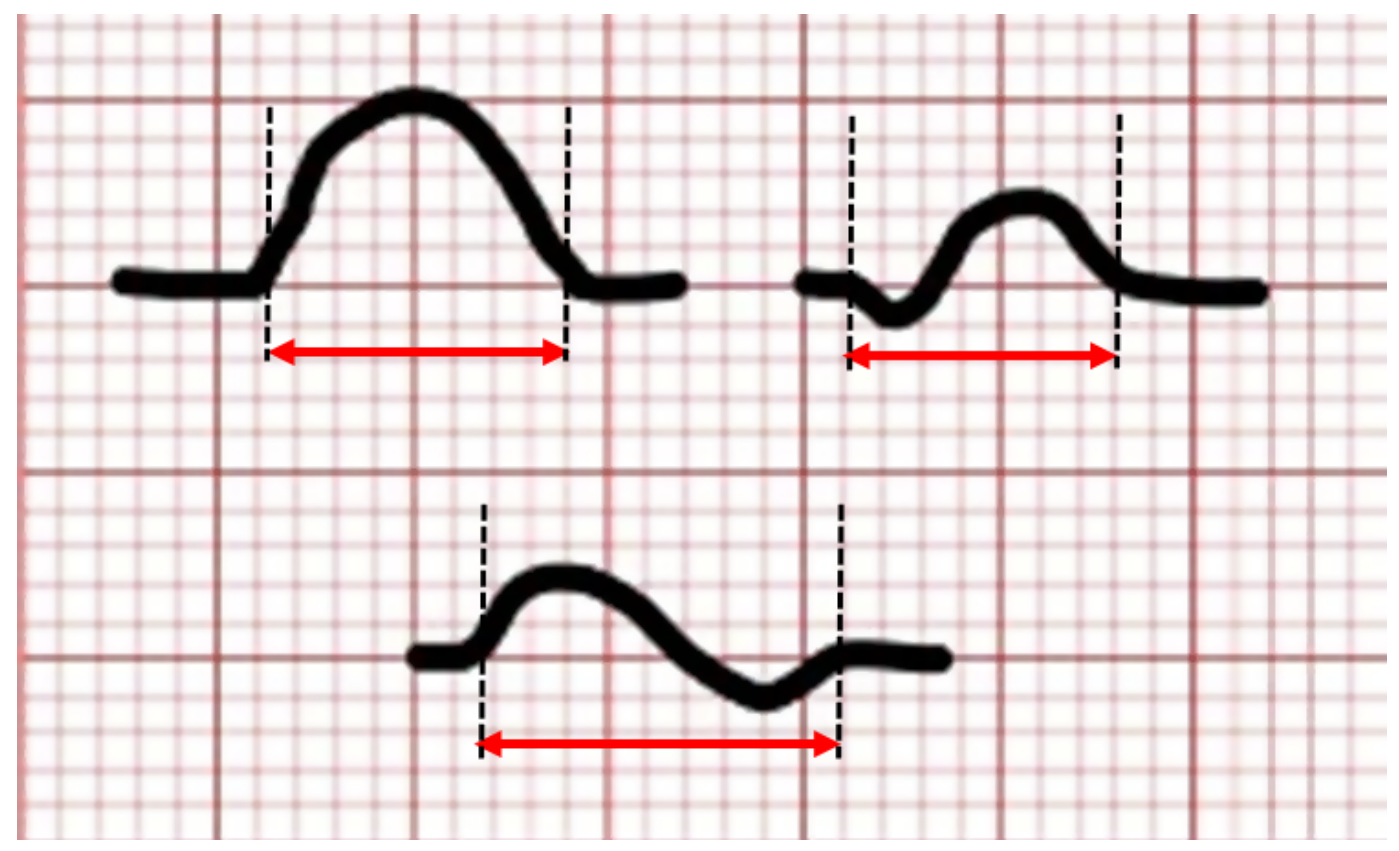

\section{$P$ wave amplitude}

$\mathrm{P}$ wave amplitude will be measured in lead II. Measurement from the onset of the $\mathrm{P}$ wave to its highest amplitude. Use previous T-P segment as baseline (end of T-wave to beginning of P-wave).

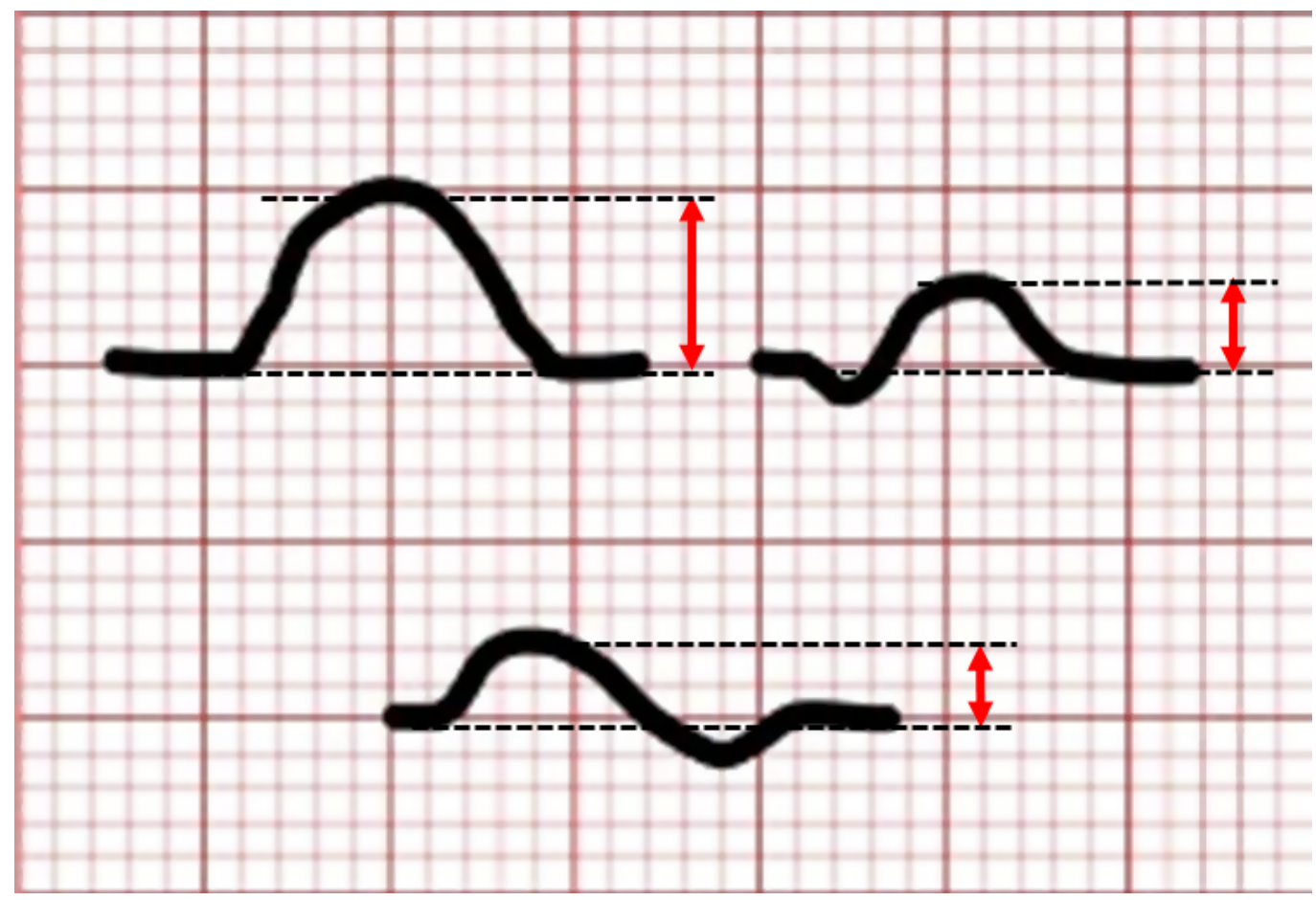




\section{PR wave duration}

PR duration will be measured in lead II. The PR interval was measured from the onset of the $\mathrm{P}$ wave to the onset of the QRS complex, defined as the initial deflection from the baseline of the PR interval.

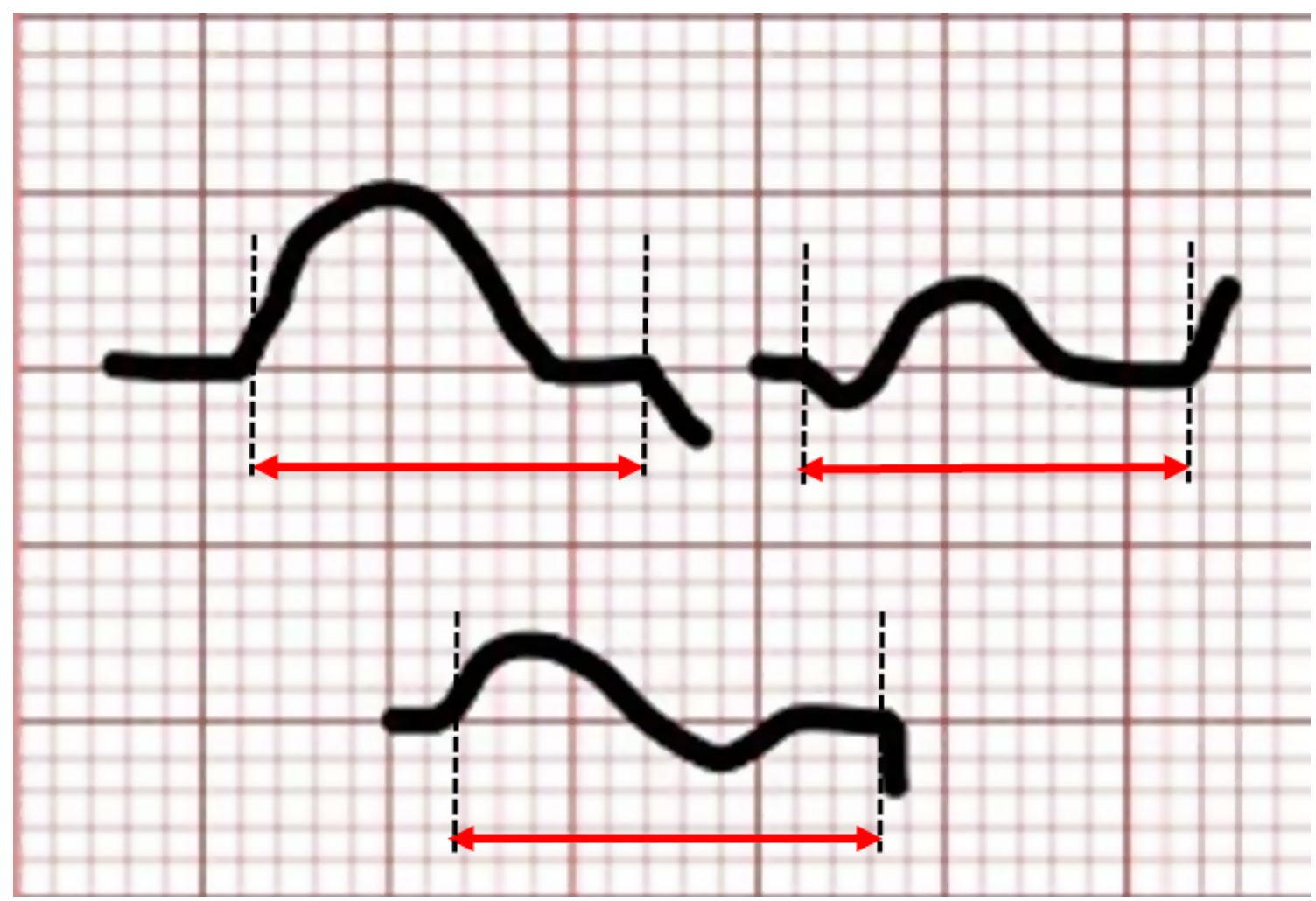




\section{QT interval}

The QT interval was measured in lead V5, and determined as the onset of the QRS complex to the end of the $\mathrm{T}$ wave; defined as the return of the $\mathrm{T}$ wave to the isoelectric baseline of the TP segment.

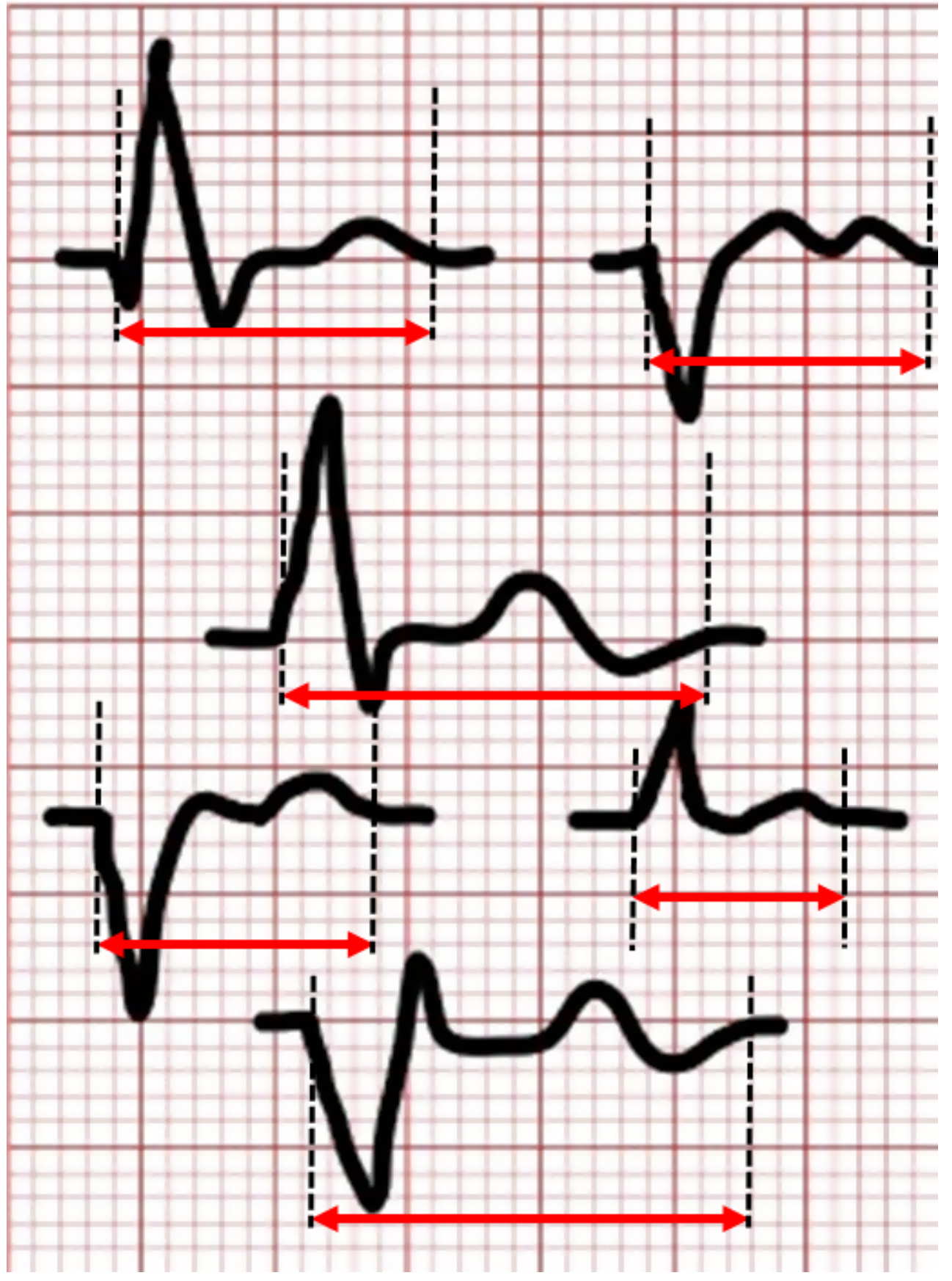


QRS duration

QRS duration was measured in lead V6, and determined as the onset of the QRS complex to the return of the complex to the isoelectric baseline of the ST segment. If in doubt regarding the isoelectric segment, use previous T-P segment as baseline (end of T-wave to beginning of P-wave).

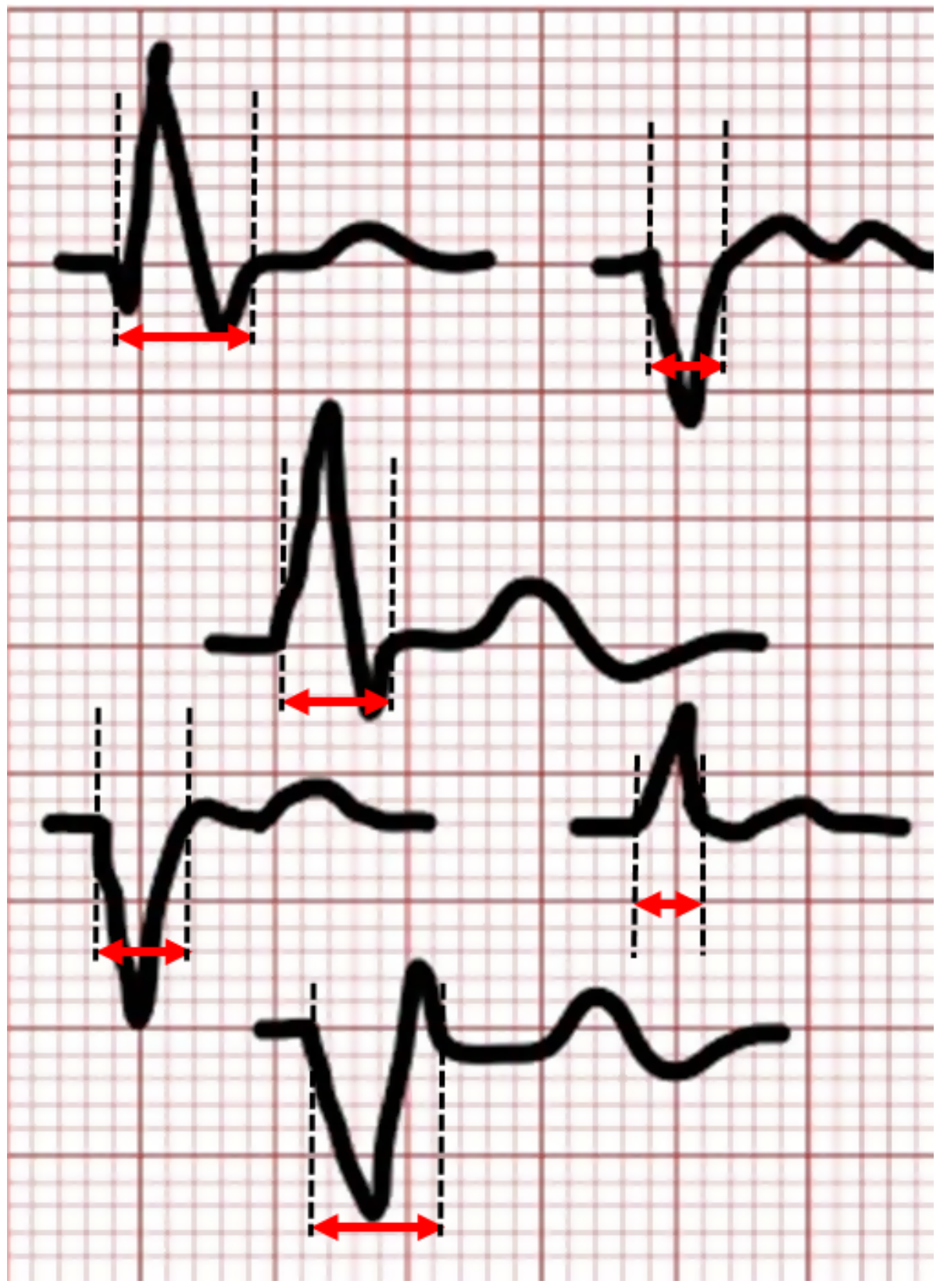


In cases when it is hard to identify the end of QRS because ST segment is sloped and/or QRS is wide and complex then tangent line method as below can be used: when the tracing becomes more horizontal than vertical.
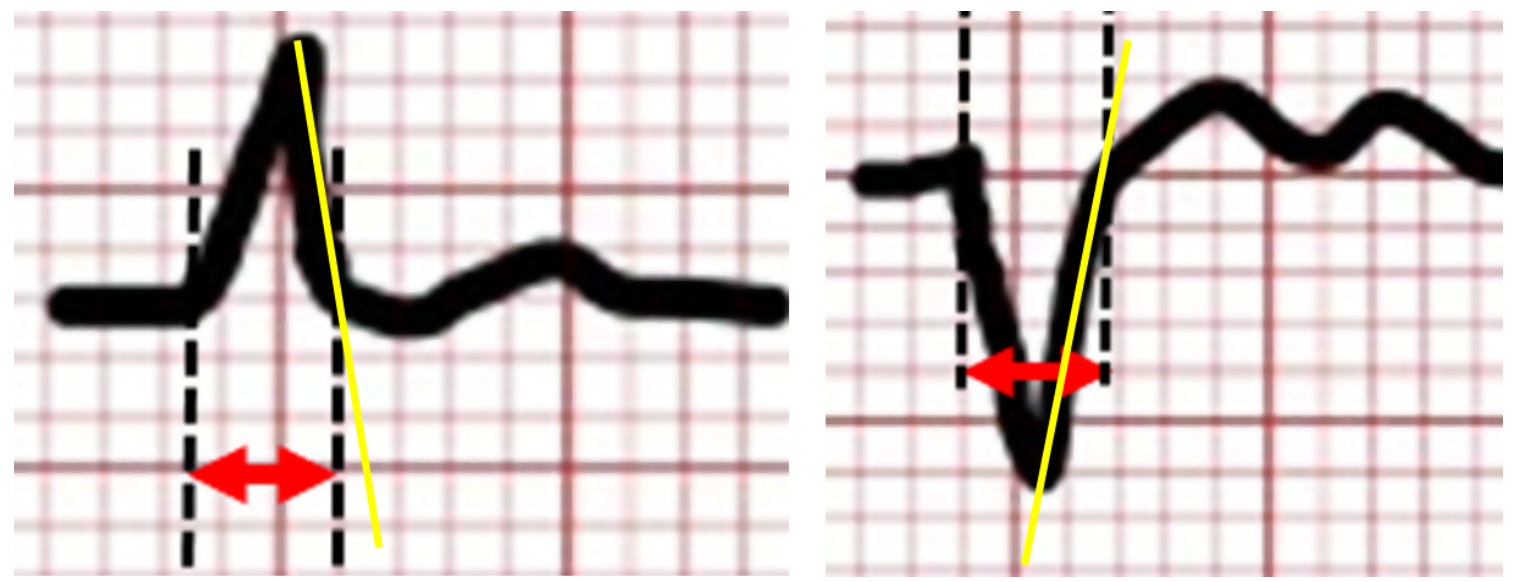

When in doubt or when two J points are visualized then the earlier J point should be used to measure QRS duration.

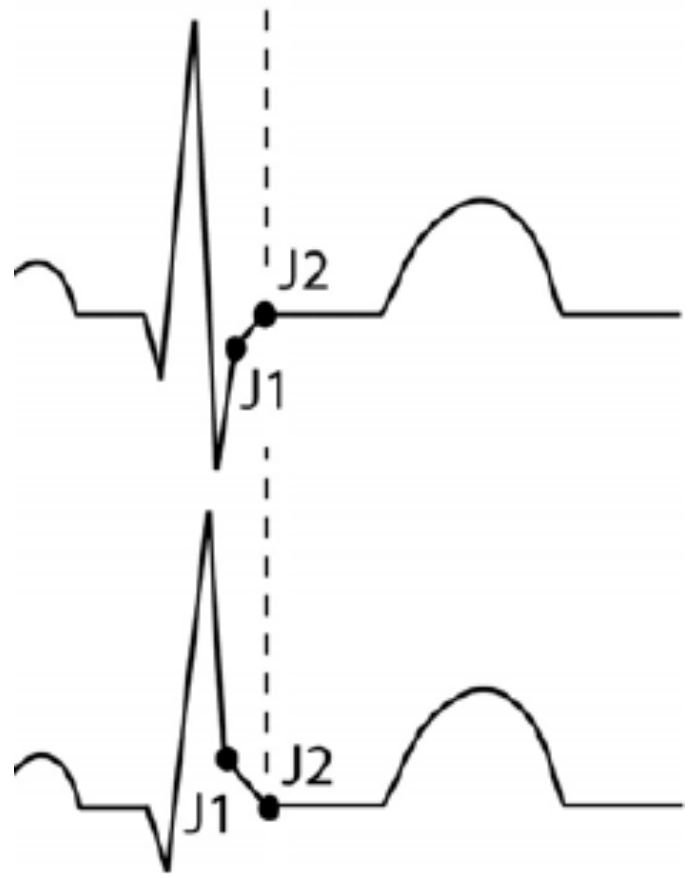




\section{T wave amplitude}

T wave amplitude will be measured in lead V5. Measurement from the onset of the T wave to its highest amplitude. In case of predominant negative $\mathrm{T}$ wave where the highest deflection is below the baseline then add a comment on the side and measure the amplitude from baseline to most lowest amplitude.

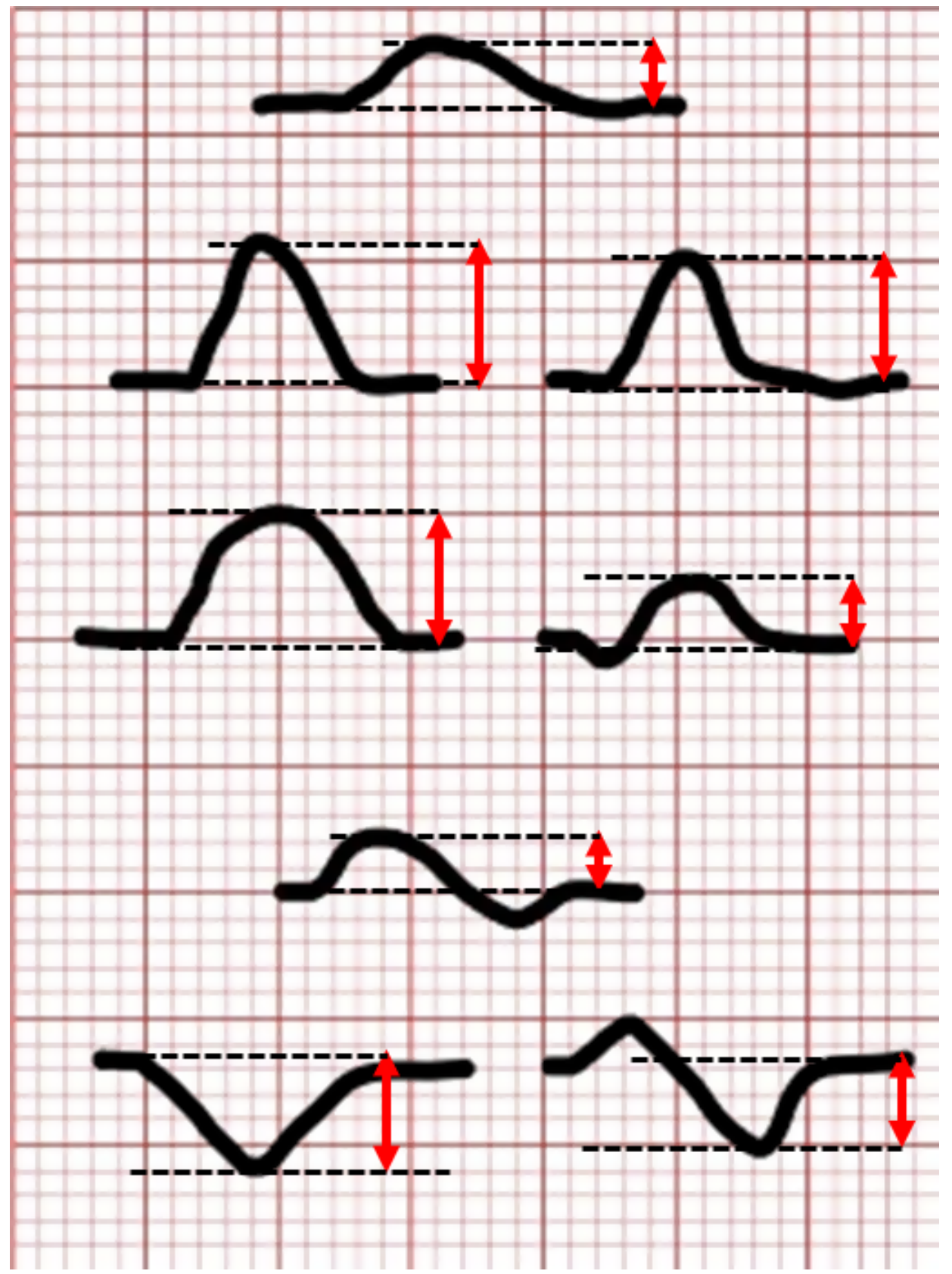




\section{T peak - $T$ end}

$\mathrm{T}$ wave amplitude will be measured in lead V5. Measurement will be conducted peak of T-wave to the offset of the $\mathrm{T}$ wave, defined as the return of the $\mathrm{T}$ wave to the isoelectric baseline (TP segment as described above).

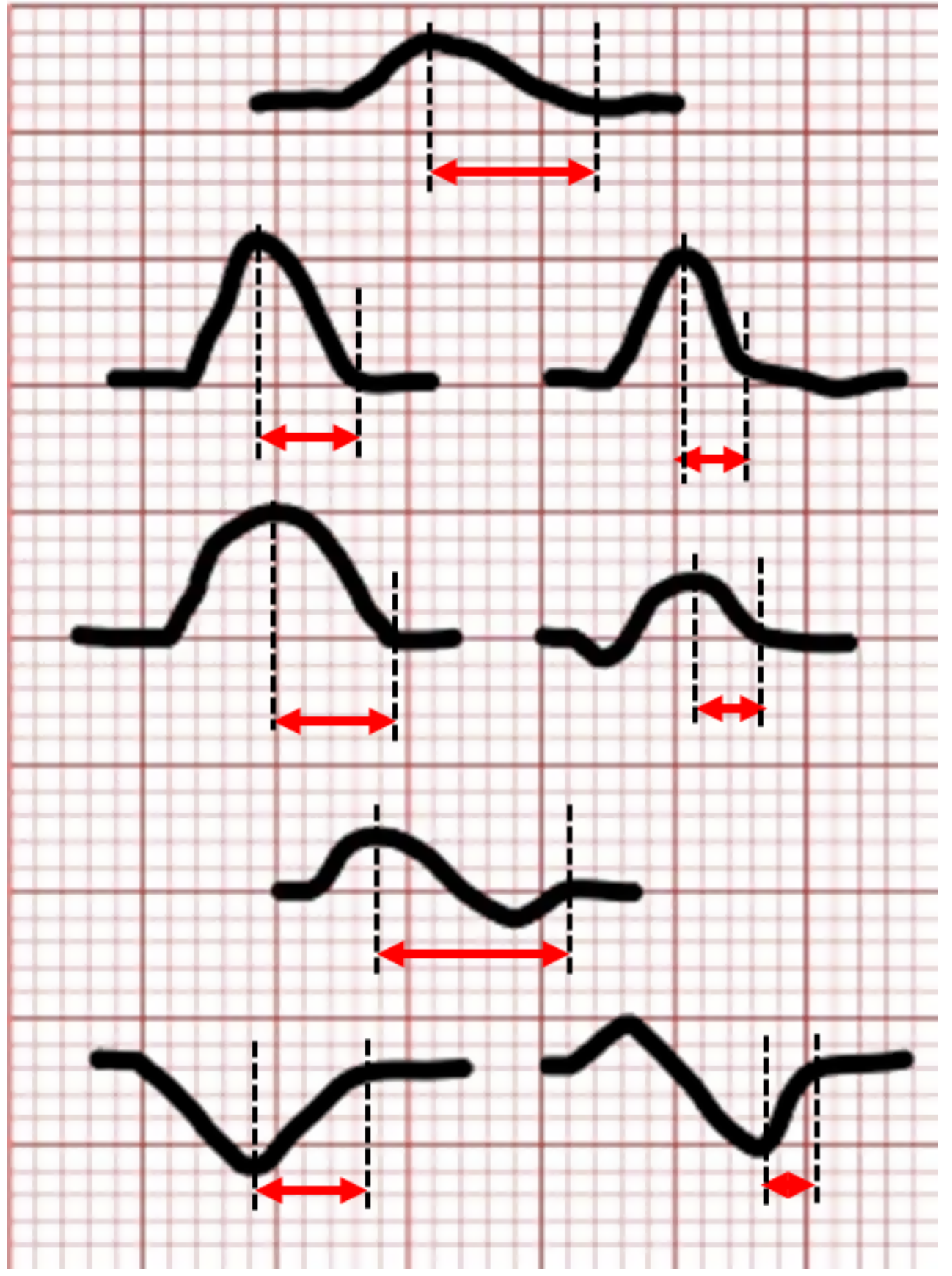


In cases when it is hard to identify the end of $\mathrm{T}$ then tangent line method as below can be used.
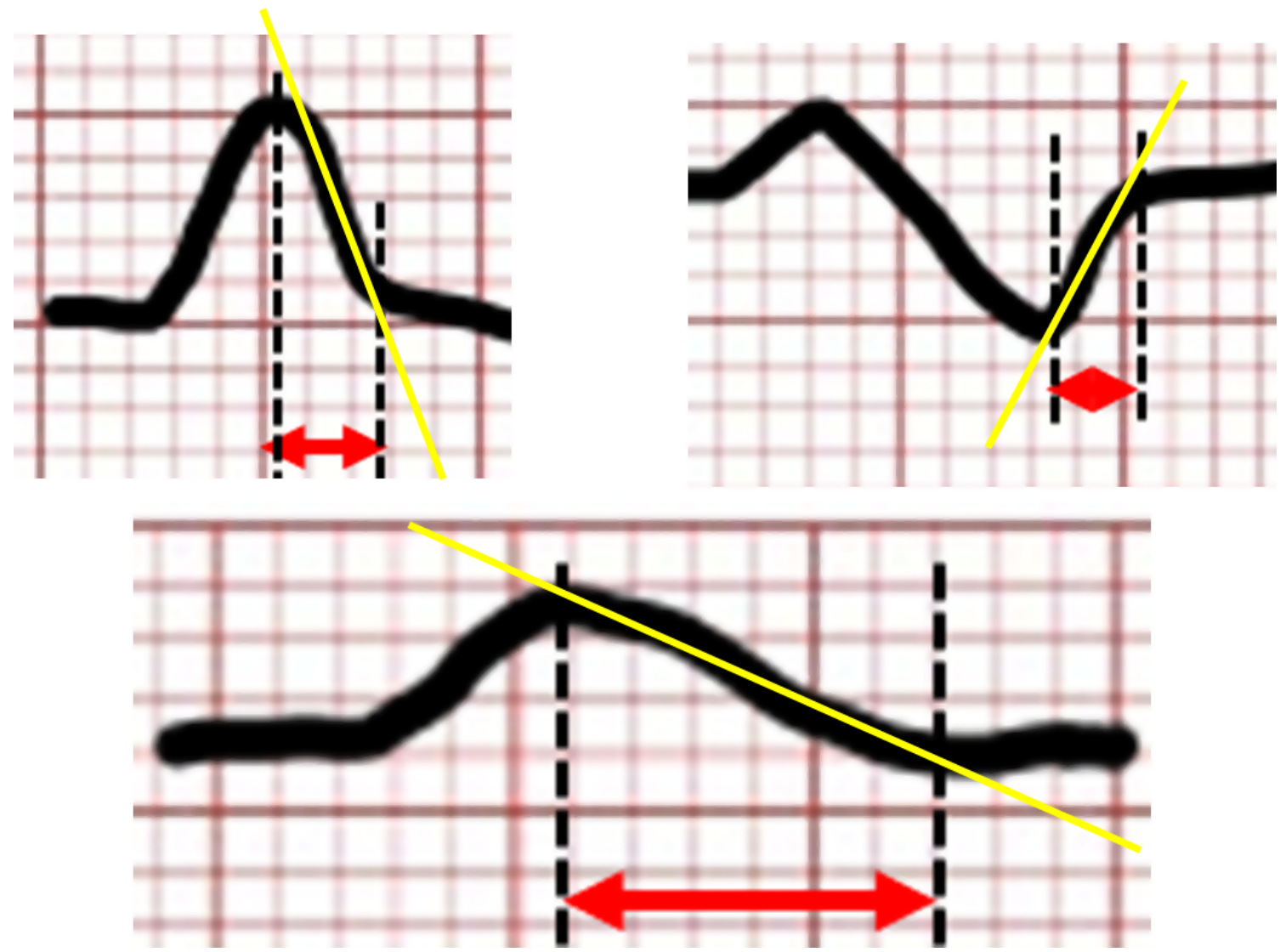

\section{$\mathbf{R}-\mathbf{R}$ intervals}

Use the lead which has the longest continuous ECG recording, which is usually at the bottom of the ECG sheet. Use preferably Lead II if available otherwise use V1. Measure the duration between all $\mathrm{R}-\mathrm{R}$ intervals. If $\mathrm{R}$ is not present then use any identical points on consecutive EKG waveforms such as the Q-Q or P-P duration. Do not measure R-R between sinus and a premature atrial or ventricular beat (defined as beat occurring $20 \%$ earlier to previous interval) and measure the subsequent waveforms. 


\section{References for ECG Measurement protocol}

Prineas RJ, Crow RS Zhang ZM. The Minnesota Code Manual of Electrocardiographic Findings. 2nd Edition, London: Springer, 2010.

Jacob Melgaard et al. Automatic J-point Location in Subjects with Electrocardiographic Early Repolarization Computing in Cardiology 2014; 41:585-588

Considerations about the polemic J point location

http://bscw.rediris.es/pub/bscw.cgi/d5046031/CONSIDERACIONES\%20ACERCA\%20 DE\%20LA\%20LOCALIZACI\%C3\%93N\%20POL\%C3\%89MICA\%20DEL\%20 PUNTO\%20J.pdf

Burke GM et al. J Electrocardiol. 2014 May-Jun;47(3):288-93. doi:

10.1016/j.jelectrocard.2014.01.004. Epub 2014 Jan 6. Assessment of reproducibility--automated and digital caliper ECG measurement in the Framingham Heart Study.

Tarek MohamedAbdelrahman The Egyptian Journal of Critical Care Medicine Volume 2, Issue 1, April 2014, Pages 19-27. Prognostic value of T peak-to-end interval for risk stratification after acute myocardial infarction

Erikssen G et al. Ann Noninvasive Electrocardiol. 2012 Apr;17(2):85-94. doi:

10.1111/j.1542-474X.2012.00493.x. The terminal part of the QT interval (T peak to $\mathrm{T}$ end): a predictor of mortality after acute myocardial infarction. 
Supplementary Table 2.2.1 Baseline characteristics by high and low PR interval, P wave duration and PR segment

\begin{tabular}{|c|c|c|c|c|c|c|c|c|c|}
\hline & \multicolumn{3}{|c|}{ PR interval } & \multicolumn{3}{|c|}{ P-wave duration } & \multicolumn{3}{|c|}{ PR segment } \\
\hline & $\begin{array}{c}\leq 163.32 \\
\mathrm{~ms}\end{array}$ & $\begin{array}{c}>163.33+ \\
\mathrm{ms}\end{array}$ & $\mathrm{p}$ value & $\begin{array}{c}\leq 104.73 \\
\mathrm{~ms}\end{array}$ & $\begin{array}{c}>104.74 \\
\mathrm{~ms}\end{array}$ & $\mathrm{p}$ value & $\begin{array}{c}\leq 55.20 \\
\mathrm{~ms}\end{array}$ & $\begin{array}{c}>55.21 \\
\mathrm{~ms}\end{array}$ & $\mathrm{p}$ value \\
\hline Age (years)* & 49,11 & 55,8 & 0.01 & 51,11 & 54,8 & 0.11 & 51,10 & 53,9 & 0.18 \\
\hline BMI $(\mathrm{kg} / \mathrm{m} 2)^{*}$ & 31,7 & 34,9 & 0.07 & 31,7 & 35,8 & 0.01 & 32,7 & 34,9 & 0.10 \\
\hline $\mathrm{SBP}(\mathrm{mm} \mathrm{Hg})^{*}$ & 133,21 & 134,25 & 0.95 & 129,21 & 138,25 & 0.07 & 135,22 & 132,24 & 0.13 \\
\hline $\mathrm{DBP}(\mathrm{mm} \mathrm{Hg})^{*}$ & 80,10 & 81,15 & 0.52 & 78,12 & 84,14 & 0.05 & 82,11 & 79,15 & 0.16 \\
\hline Heart rate (beats/min)* & 75,17 & 71,13 & 0.27 & 73,16 & 73,13 & 0.53 & 75,16 & 72,14 & 0.28 \\
\hline Male gender & $27,42 \%$ & $45,63 \%$ & 0.01 & $31,44 \%$ & $41,64 \%$ & 0.02 & $33,52 \%$ & $39,55 \%$ & 0.70 \\
\hline Caucasian & $37,58 \%$ & $40,56 \%$ & 0.86 & $44,62 \%$ & $33,52 \%$ & 0.22 & $39,61 \%$ & $38,54 \%$ & 0.39 \\
\hline Hypertension & $54,84 \%$ & $64,90 \%$ & 0.31 & $57,80 \%$ & $61,95 \%$ & 0.01 & $55,86 \%$ & $63,89 \%$ & 0.63 \\
\hline Prior MI & $26,41 \%$ & $36,51 \%$ & 0.24 & $31,44 \%$ & $31,48 \%$ & 0.58 & $32,50 \%$ & $30,42 \%$ & 0.37 \\
\hline Diabetes & $18,28 \%$ & $25,35 \%$ & 0.38 & $20,28 \%$ & $23,36 \%$ & 0.33 & $17,27 \%$ & $26,37 \%$ & 0.21 \\
\hline Stroke & $13,20 \%$ & $10,14 \%$ & 0.34 & $15,21 \%$ & $8,13 \%$ & 0.18 & $10,16 \%$ & $13,18 \%$ & 0.68 \\
\hline Arrhythmia & $22,34 \%$ & $23,32 \%$ & 0.81 & $25,35 \%$ & $20,31 \%$ & 0.63 & $20,31 \%$ & $25,35 \%$ & 0.63 \\
\hline B blocker & $43,67 \%$ & $55,78 \%$ & 0.18 & $51,72 \%$ & $47,73 \%$ & 0.83 & $42,66 \%$ & $56,79 \%$ & 0.09 \\
\hline $\mathrm{CCB}$ & $13,20 \%$ & $19,27 \%$ & 0.38 & $10,14 \%$ & $22,34 \%$ & 0.01 & $13,20 \%$ & $19,27 \%$ & 0.38 \\
\hline ACEI or ARB & $38,59 \%$ & $51,72 \%$ & 0.13 & $39,55 \%$ & $50,78 \%$ & 0.01 & $44,69 \%$ & $45,63 \%$ & 0.51 \\
\hline Statin & $41,64 \%$ & $43,61 \%$ & 0.68 & $45,63 \%$ & $39,61 \%$ & 0.77 & $41,64 \%$ & $43,61 \%$ & 0.68 \\
\hline Aspirin & $35,55 \%$ & $46,65 \%$ & 0.23 & $43,61 \%$ & $38,59 \%$ & 0.89 & $34,53 \%$ & $47,66 \%$ & 0.12 \\
\hline Diuretics & $24,38 \%$ & $34,48 \%$ & 0.22 & $26,37 \%$ & $32,50 \%$ & 0.12 & $21,33 \%$ & $37,52 \%$ & 0.02 \\
\hline
\end{tabular}

Abbreviations: BMI - Body mass index, SBP- Systolic Blood Pressure, DBP - Diastolic Blood Pressure, MI - Myocardial Infarction, CCB - Calcium Channel Blocker, ACE - Angiotensin-Converting Enzyme Inhibitor, ARB - Angiotensin II Receptor Blocker.

For each variable counts and column percentage has been reported except for where indicated * where the mean and standard deviation (SD) are reported 
Supplementary Table 2.2.2 Distribution of urinary cotinine levels by self-reported active smoking status among all participants

\begin{tabular}{lcc}
\hline Smoking status & \multicolumn{2}{c}{ Cotinine } \\
& $\begin{array}{c}\leq 50 \mathrm{ng} / \mathrm{ml} \\
(\mathrm{N}=73,54 \%)\end{array}$ & $\begin{array}{c}>50 \mathrm{ng} / \mathrm{ml} \\
(\mathrm{N}=63,46 \%)\end{array}$ \\
\hline Current smoker $(53,39 \%)$ & $2,3 \%$ & $51,81 \%$ \\
Former smoker $(47,35 \%)$ & $38,52 \%$ & $9,14 \%$ \\
Never smoker $(36,26 \%)$ & $33,45 \%$ & $3,5 \%$ \\
\hline
\end{tabular}


Supplementary Table 2.2.3 Comparison among participants stratified by self-reported smoking status or by cotinine level of (A) urinary cotinine and catecholamines (median [interquartile range], creatinine-normalized), and (B) high and low PR interval, P wave duration and PR segment

(A)

\begin{tabular}{|c|c|c|c|c|c|c|c|c|c|}
\hline & & \multicolumn{2}{|l|}{$\begin{array}{l}\text { Cotinine } \\
\text { (ng/g) }\end{array}$} & \multicolumn{2}{|l|}{$\begin{array}{l}\text { Dopamine } \\
\quad(\mu \mathrm{g} / \mathrm{g})\end{array}$} & \multicolumn{2}{|c|}{$\begin{array}{l}\text { Epinephrine } \\
\quad(\mu \mathrm{g} / \mathrm{g})\end{array}$} & \multicolumn{2}{|c|}{$\begin{array}{l}\text { Norepinephrine } \\
(\mu \mathrm{g} / \mathrm{g})\end{array}$} \\
\hline & & & $\begin{array}{c}\mathrm{p} \\
\text { value }\end{array}$ & & $\begin{array}{c}\mathrm{p} \\
\text { value }\end{array}$ & & $\begin{array}{c}\mathrm{p} \\
\text { value }\end{array}$ & & $\begin{array}{c}\mathrm{p} \\
\text { value }\end{array}$ \\
\hline \multirow{3}{*}{$\begin{array}{l}\text { Smoking } \\
\text { status }\end{array}$} & $\begin{array}{l}\text { Active } \\
\text { smoker }\end{array}$ & $\begin{array}{c}827.1 \\
{[527.3-1445.3]}\end{array}$ & $<0.001$ & $\begin{array}{c}176.0 \\
{[146.5-225.8]}\end{array}$ & 0.24 & $\begin{array}{c}5.7 \\
{[3.3-10.8]}\end{array}$ & 0.31 & $\begin{array}{c}42.5 \\
{[30.7-60.3]}\end{array}$ & 0.07 \\
\hline & $\begin{array}{l}\text { Former- } \\
\text { smoker* }\end{array}$ & $\begin{array}{c}3.7 \\
{[1.3-18.9]}\end{array}$ & - & $\begin{array}{c}167.1 \\
{[135.6-204.9]}\end{array}$ & - & $\begin{array}{c}4.2 \\
{[2.6-8.5]}\end{array}$ & - & $\begin{array}{c}36.2 \\
{[26.4-51.4]}\end{array}$ & - \\
\hline & $\begin{array}{l}\text { Never } \\
\text { smoker }\end{array}$ & $\begin{array}{c}1.6 \\
{[0.9-4.0]}\end{array}$ & $<0.001$ & $\begin{array}{c}139.7 \\
{[114.3-169.7]}\end{array}$ & 0.001 & $\begin{array}{c}3.7 \\
{[1.5-6.5]}\end{array}$ & 0.03 & $\begin{array}{c}32.4 \\
{[19.0-49.2]}\end{array}$ & 0.02 \\
\hline \multirow{2}{*}{ Cotinine } & $\leq 50 \mathrm{ng} / \mathrm{ml}$ & $\begin{array}{c}1.8 \\
{[1.0-4.2]}\end{array}$ & \multirow{2}{*}{$<0.001$} & $\begin{array}{c}160.0 \\
{[112.4-189.1]}\end{array}$ & \multirow{2}{*}{0.003} & $\begin{array}{c}4.1 \\
{[2.1-6.9]}\end{array}$ & 0.08 & $\begin{array}{c}33.4 \\
{[22.7-50.6]}\end{array}$ & \multirow{2}{*}{0.02} \\
\hline & $>50 \mathrm{ng} / \mathrm{ml}$ & $\begin{array}{c}827.1 \\
{[522.8-1596.0]}\end{array}$ & & $\begin{array}{c}175.8 \\
{[142.0-227.9]}\end{array}$ & & $\begin{array}{c}5.9 \\
{[3.1-9.2]}\end{array}$ & 0.00 & $\begin{array}{c}42.5 \\
{[30.4-56.4]}\end{array}$ & \\
\hline
\end{tabular}


(B)

\begin{tabular}{|c|c|c|c|c|c|c|c|c|c|c|}
\hline & & \multicolumn{3}{|c|}{ PR interval } & \multicolumn{3}{|c|}{$\mathrm{P}$ wave duration } & \multicolumn{3}{|c|}{ PR segment } \\
\hline & & $\leq 163.32 \mathrm{~ms}$ & $>163.33 \mathrm{~ms}$ & $\begin{array}{c}\mathrm{p} \\
\text { value }\end{array}$ & $\leq 104.73 \mathrm{~ms}$ & $104.74 \mathrm{~ms}$ & $\begin{array}{c}\mathrm{p} \\
\text { value }\end{array}$ & $\leq 55.20 \mathrm{~ms}$ & $55.21 \mathrm{~ms}$ & $\begin{array}{c}\mathrm{p} \\
\text { value }\end{array}$ \\
\hline \multirow{3}{*}{$\begin{array}{l}\text { Smoking } \\
\text { status }\end{array}$} & $\begin{array}{l}\text { Current } \\
\text { smoker }\end{array}$ & $25,39 \%$ & $28,39 \%$ & \multirow{3}{*}{0.76} & $34,48 \%$ & $19,30 \%$ & \multirow{3}{*}{0.08} & $26,40 \%$ & $27,38 \%$ & \multirow{3}{*}{0.58} \\
\hline & $\begin{array}{l}\text { Former } \\
\text { smoker }\end{array}$ & $24,38 \%$ & $23,32 \%$ & & $20,28 \%$ & $27,42 \%$ & & $24,38 \%$ & $23,32 \%$ & \\
\hline & $\begin{array}{l}\text { Never } \\
\text { smoker }\end{array}$ & $15,23 \%$ & $20,28 \%$ & & $17,24 \%$ & $18,28 \%$ & & $14,22 \%$ & $21,30 \%$ & \\
\hline \multirow{2}{*}{ Cotinine } & $\leq 50 \mathrm{ng} / \mathrm{ml}$ & $34,53 \%$ & $38,54 \%$ & \multirow{2}{*}{0.96} & $34,48 \%$ & $38,59 \%$ & \multirow{2}{*}{0.18} & $32,50 \%$ & $40,56 \%$ & \multirow{2}{*}{0.49} \\
\hline & $>50 \mathrm{ng} / \mathrm{ml}$ & $30,47 \%$ & $33,46 \%$ & & $37,52 \%$ & $26,41 \%$ & & $32,50 \%$ & $31,44 \%$ & \\
\hline
\end{tabular}


Supplementary Table 2.2.4 Association between creatinine-normalized log transformed cotinine and parent catecholamines, their intermediate and final metabolites, and their metabolism, denoted by the ratios of intermediate: parent and final: intermediate metabolites

\begin{tabular}{|c|c|c|c|c|c|c|c|c|c|c|c|}
\hline & & \multicolumn{2}{|c|}{ Parent } & \multicolumn{2}{|c|}{ Intermediate } & \multicolumn{2}{|c|}{ Intermediate / Parent } & \multicolumn{2}{|c|}{ Final } & \multicolumn{2}{|c|}{ Final / Intermediate } \\
\hline & & $\beta$ & $\mathrm{p}$ value & $\beta$ & $\mathrm{p}$ value & $\beta$ & $\mathrm{p}$ value & $\beta$ & $\mathrm{p}$ value & $\beta$ & $\mathrm{p}$ value \\
\hline & $\mathrm{DA}$ & 0.25 & $<0.01$ & 0.36 & $<0.01$ & 0.19 & $\mathbf{0 . 0 3}$ & -0.01 & 0.95 & -0.37 & $<0.01$ \\
\hline Cotinine & $\mathrm{NE}$ & 0.22 & 0.01 & 019 & 0.03 & -0.06 & 0.47 & -0.14 & 0.11 & -0.28 & $<0.01$ \\
\hline & $\mathrm{EP}$ & 0.11 & 0.19 & 0.37 & $<0.01$ & 0.10 & 0.27 & -0.14 & 0.11 & -0.37 & $<\mathbf{0 . 0 1}$ \\
\hline
\end{tabular}

\begin{tabular}{lll} 
Parent & Intermediate & Final \\
\hline DA: Dopamine & 3-methoxytyramine & Homovanillic acid \\
NE: Norepinephrine & Normetanephrine & Vanillylmandelic acid \\
EP: Epinephrine & Metanephrine & Vanillylmandelic acid
\end{tabular}


Supplementary Table 2.2.5 Associations of QRS duration, QT interval and corrected QT with urinary cotinine in unadjusted and adjusted linear regressions

Urinary cotinine was normalized by urine creatinine and log-transformed

\begin{tabular}{lcccccc}
\hline & \multicolumn{2}{c}{ QRS } & \multicolumn{2}{c}{ QT } & \multicolumn{2}{c}{ Corrected QT } \\
& $\beta$ & $\mathrm{p}$ value & $\beta$ & $\mathrm{p}$ value & $\beta$ & $\mathrm{p}$ value \\
\hline Unadjusted Cotinine & 0.32 & 0.80 & 4.71 & 0.11 & 3.48 & 0.14 \\
*Adjusted Cotinine & -0.34 & 0.78 & 3.33 & 0.78 & 0.11 & 0.13 \\
\hline
\end{tabular}

* models adjusted for diastolic blood pressure and prior myocardial infarction (QRS); age, heart rate, systolic blood pressure, and calcium channel blocker (QT); age $(\mathrm{QTc})$ 
Supplementary Table 2.2.6 Association between catecholamine and atrial or atrioventricular conduction determined by unadjusted and adjusted linear regressions

Catecholamines were creatinine-normalized and log-transformed

\begin{tabular}{|c|c|c|c|c|c|c|c|}
\hline & & \multicolumn{2}{|c|}{ PR interval } & \multicolumn{2}{|c|}{ P wave } & \multicolumn{2}{|c|}{ PR segment } \\
\hline & & $\beta$ & $\mathrm{p}$ value & $\beta$ & $\mathrm{p}$ value & $\beta$ & $\mathrm{p}$ value \\
\hline \multirow{3}{*}{ Unadjusted } & Dopamine & -17.13 & $<0.01$ & -8.45 & $<0.01$ & -8.68 & 0.04 \\
\hline & Epinephrine & -5.47 & 0.02 & -2.8 & 0.04 & -2.66 & 0.16 \\
\hline & Norepinephrine & -7.31 & 0.11 & -4.79 & 0.08 & -2.52 & 0.49 \\
\hline \multirow{3}{*}{ *Adjusted } & Dopamine & -12.3 & 0.02 & -3.56 & 0.28 & -8.51 & 0.04 \\
\hline & Epinephrine & -6.62 & $<\mathbf{0 . 0 1}$ & -2.73 & 0.06 & -2.53 & 0.19 \\
\hline & Norepinephrine & -10.16 & 0.02 & -3.39 & 0.18 & -2.69 & 0.46 \\
\hline
\end{tabular}

* models adjust for age and gender (PR interval); body mass index, gender, hypertension, calcium channel blocker, ACE-inhibitor or angiotensin II receptor blocker (P duration); and diuretics (PR segment) 
Supplementary Table 3.1 Baseline characteristics

\begin{tabular}{|c|c|c|c|c|c|c|c|c|c|}
\hline Smoker ID & Age & Gender & Race & $\begin{array}{l}\text { Body Mass } \\
\text { Index }\end{array}$ & $\begin{array}{c}\beta \text {-Blocker } \\
\text { protocol }\end{array}$ & $\begin{array}{c}\text { Sham- } \\
\text { protocol }\end{array}$ & $\begin{array}{l}\text { Pack- } \\
\text { Years }\end{array}$ & Cigarette brand & $\begin{array}{c}\text { Nicotine } \\
\text { content }(\mathrm{mg})\end{array}$ \\
\hline 1 & 26 & Male & White & 31 & Yes & Yes & 9 & L\&M & 0.8 \\
\hline 2 & 28 & Male & Black & 29 & Yes & - & 36 & L\&M & 0.9 \\
\hline 3 & 23 & Female & Black & 29 & Yes & Yes & 5 & L\&M & 0.9 \\
\hline 4 & 32 & Male & Black & 23 & - & Yes & 29 & Newport & 0.8 \\
\hline 5 & 31 & Male & White & 37 & - & - & 15 & Marlboro & 1.1 \\
\hline 6 & 39 & Female & White & 25 & - & - & 22 & Pall Mall & 0.9 \\
\hline 7 & 23 & Male & White & 24 & Yes & Yes & 7 & L\&M & 0.9 \\
\hline 8 & 22 & Male & Black & 22 & - & - & 6 & L\&M & 0.8 \\
\hline 9 & 25 & Male & White & 24 & - & - & 7 & Camel & 0.8 \\
\hline 10 & 25 & Female & White & 30 & - & - & 11 & USA Gold Reds & 0.8 \\
\hline 11 & 52 & Male & White & 25 & Yes & - & 22 & High Card & 0.8 \\
\hline 12 & 42 & Female & White & 29 & Yes & Yes & 60 & High Card & 0.8 \\
\hline 13 & 53 & Female & White & 34 & Yes & - & 42 & Pall Mall & 1.1 \\
\hline 14 & 43 & Male & White & 30 & Yes & - & 18 & American Spirit & 0.6 \\
\hline 15 & 49 & Female & Other & 21 & Yes & - & 35 & High Card & 0.8 \\
\hline 16 & 62 & Male & Black & 28 & Yes & Yes & 20 & Newport & 0.8 \\
\hline 17 & 61 & Male & White & 21 & - & Yes & 48 & Kool & 0.6 \\
\hline 18 & 39 & Male & White & 26 & - & Yes & 25 & Marlboro & 1.0 \\
\hline 19 & 43 & Female & White & 32 & - & Yes & 29 & Camel & 1.0 \\
\hline 20 & 28 & Male & Other & 34 & - & Yes & 14 & Pall Mall & 0.9 \\
\hline
\end{tabular}


Supplementary Table 3.2 Correlation between Area under the Curve of root mean squared of successive differences (RMSSD) and rest of ECG waveform parameters

\begin{tabular}{lcc}
\hline & $\begin{array}{c}\text { Cigarette-Days and } \\
\text { Non-smokers } \\
\mathrm{N}=29\end{array}$ & $\begin{array}{c}\text { Cigarette-Days, } \\
\text { Nicotine Days } \\
\text { and Non-smokers } \\
\text { N=49 }\end{array}$ \\
\hline Heart Rate & $\mathbf{- 0 . 4 5 9 * *}$ & $\mathbf{- 0 . 4 3 6 * *}$ \\
ST height & $\mathbf{0 . 4 3 0 *}$ & 0.225 \\
PR interval & -0.004 & 0.184 \\
PR segment & 0.164 & 0.026 \\
P duration & -0.109 & 0.190 \\
P amplitude & 0.076 & 0.174 \\
QTc interval & 0.192 & -0.002 \\
QRS duration & -0.384 & -0.217 \\
Q amplitude & 0.462 & 0.213 \\
\hline
\end{tabular}


Potential participant screened (Healthy, $18-65$ years, active smokers, Nicotine $0.6-1.2 \mathrm{ng} / \mathrm{ml}$ ). Participant found eligible and consented

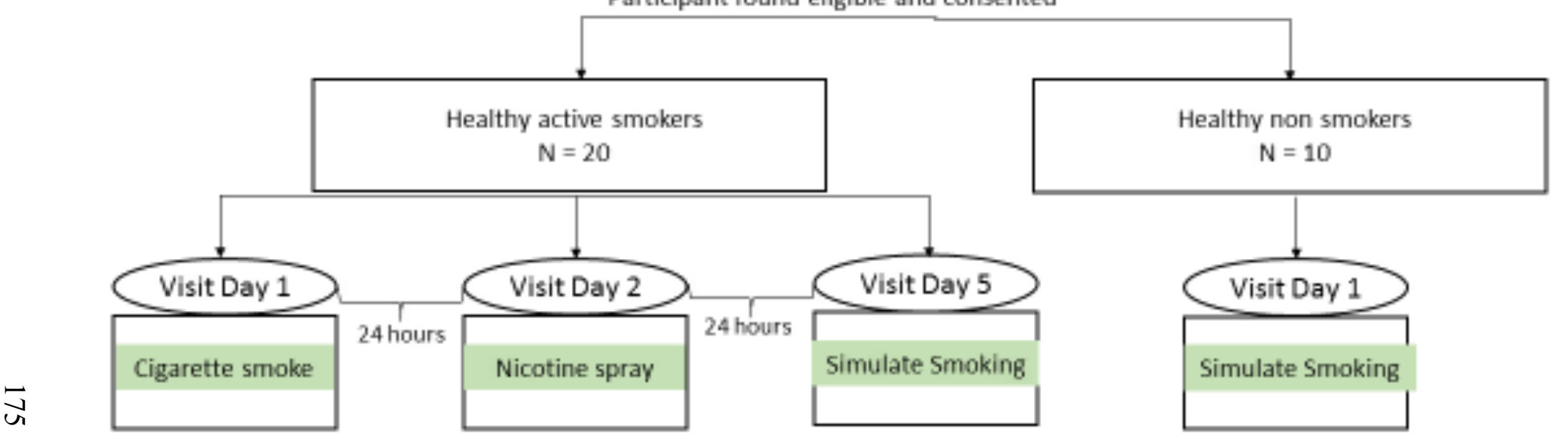

10 participants will complata additional two day study

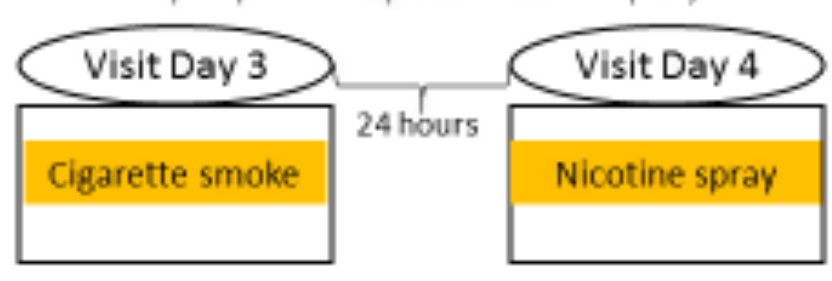

Visit days 1 and 2: Baseline 5 minutes, 20 minutes during exposure and 5 mins post exposure

Visit days 3 and 4 ; Oral $80 \mathrm{mg}$ propranolol, 2 hour wait, Baseline 5 minutes, 20 minutes during exposure and 5 mins post exposure 
(B)

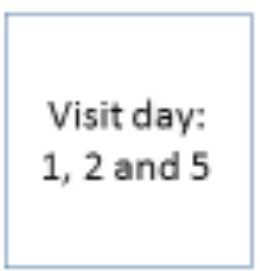

\begin{tabular}{|c|c|c|c|c|}
\hline & \multicolumn{3}{|c|}{ Continuous 12 lead Electrocardiogram } & \\
\hline $\begin{array}{c}\text { Resting } \\
\text { vitals, } \\
\text { Blood draw }\end{array}$ & $\begin{array}{l}\text { Pre-exposure } \\
5 \text { mins } \\
\text { Lying } \\
\text { Indoors }\end{array}$ & $\begin{array}{c}\text { Exposure* } \\
20 \text { mins } \\
\text { Sitting } \\
\text { Outdoors }\end{array}$ & $\begin{array}{l}\text { Post-exposure } \\
5 \text { mins } \\
\text { Lying } \\
\text { Indoors }\end{array}$ & $\begin{array}{c}\text { Resting } \\
\text { vitals, } \\
\text { Blood draw }\end{array}$ \\
\hline
\end{tabular}

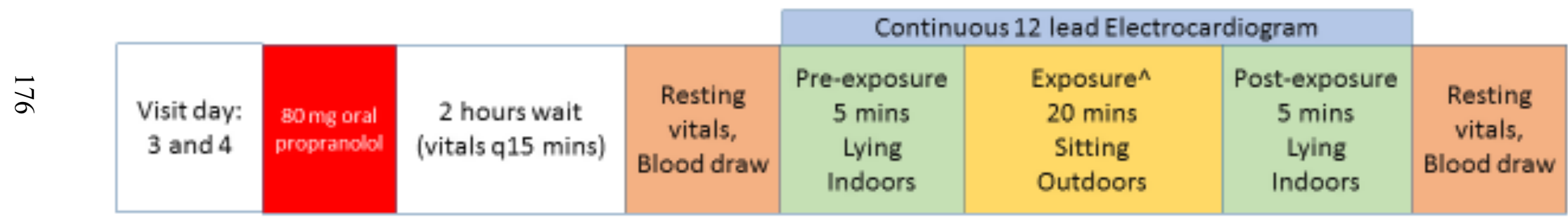

Exposure

"Day 1: Cigarette Smoking; Day 2: Oral Nicotine Spray; Day 5: Sham Smoking

^Day 3: Cigarette smoking; Day 4: Oral Nicotine Spray

Supplementary Figure 3.1 A) Flow chart to show the study design and enrollment B) Schematic Figure to illustrate the study visit procedures on different days 


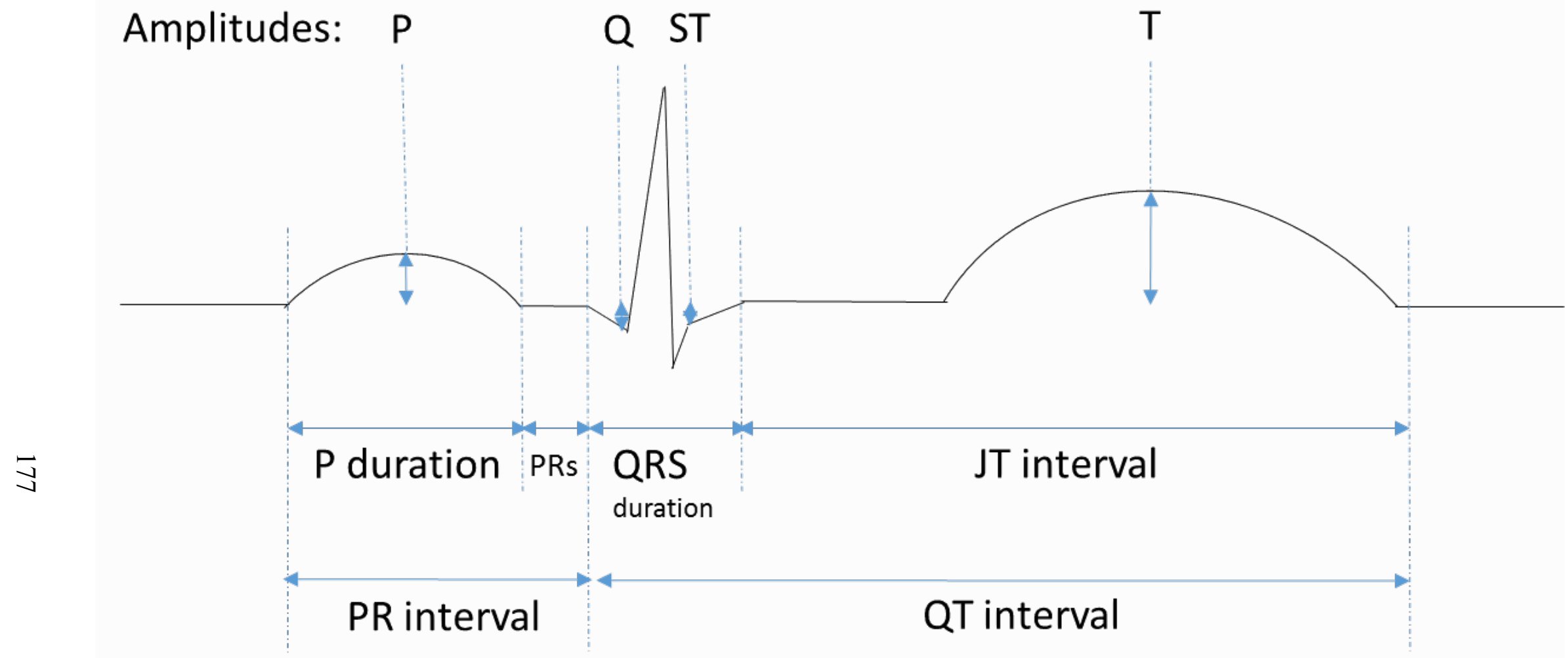

Supplementary Figure 3.2 ECG morphological variables measured from Lead II 


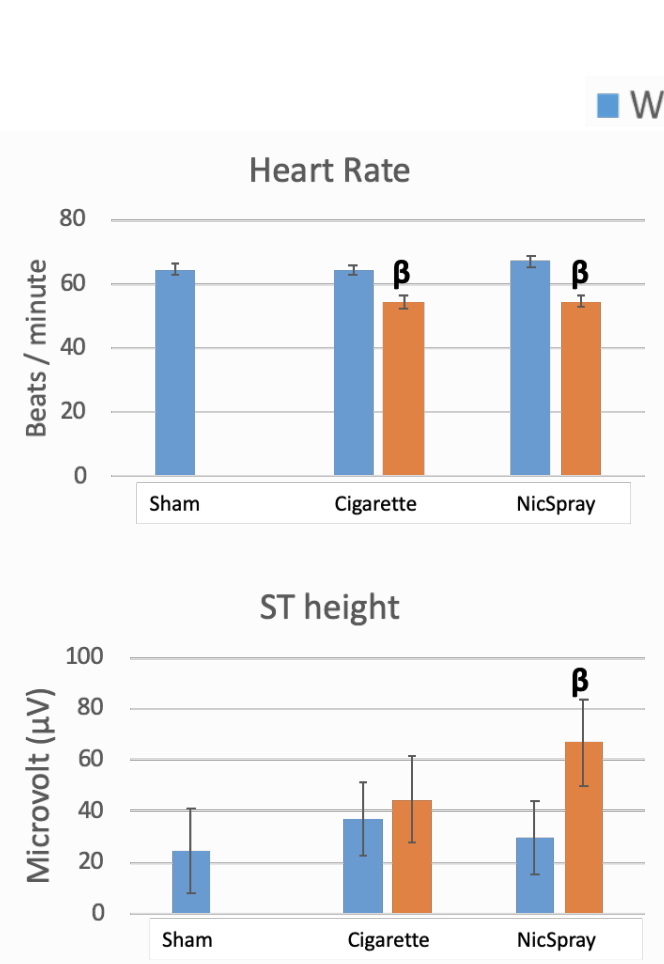

$\star:<0.05$ vs Sham

Without BB With BB $\begin{aligned} & \#:<0.05 \text { vs Nicotine } \\ & \beta:<0.05 \text { vs non-Beta-blocker }\end{aligned}$

RMSSD
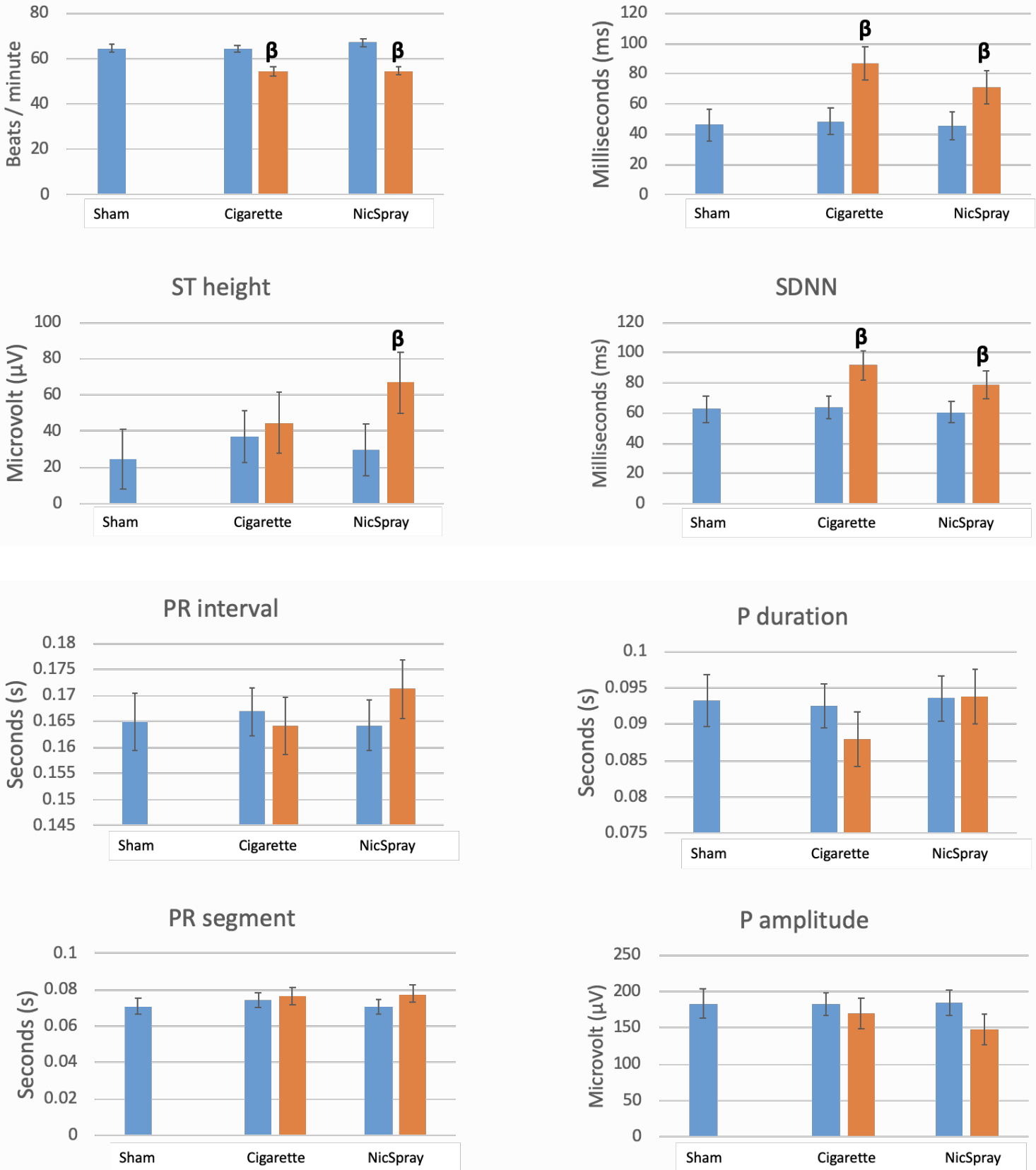


\section{$\star:<0.05$ vs Sham \\ Without BB With BB\#: $<0.05$ vs Nicotine \\ $\beta:<0.05$ vs non-Beta-blocker}
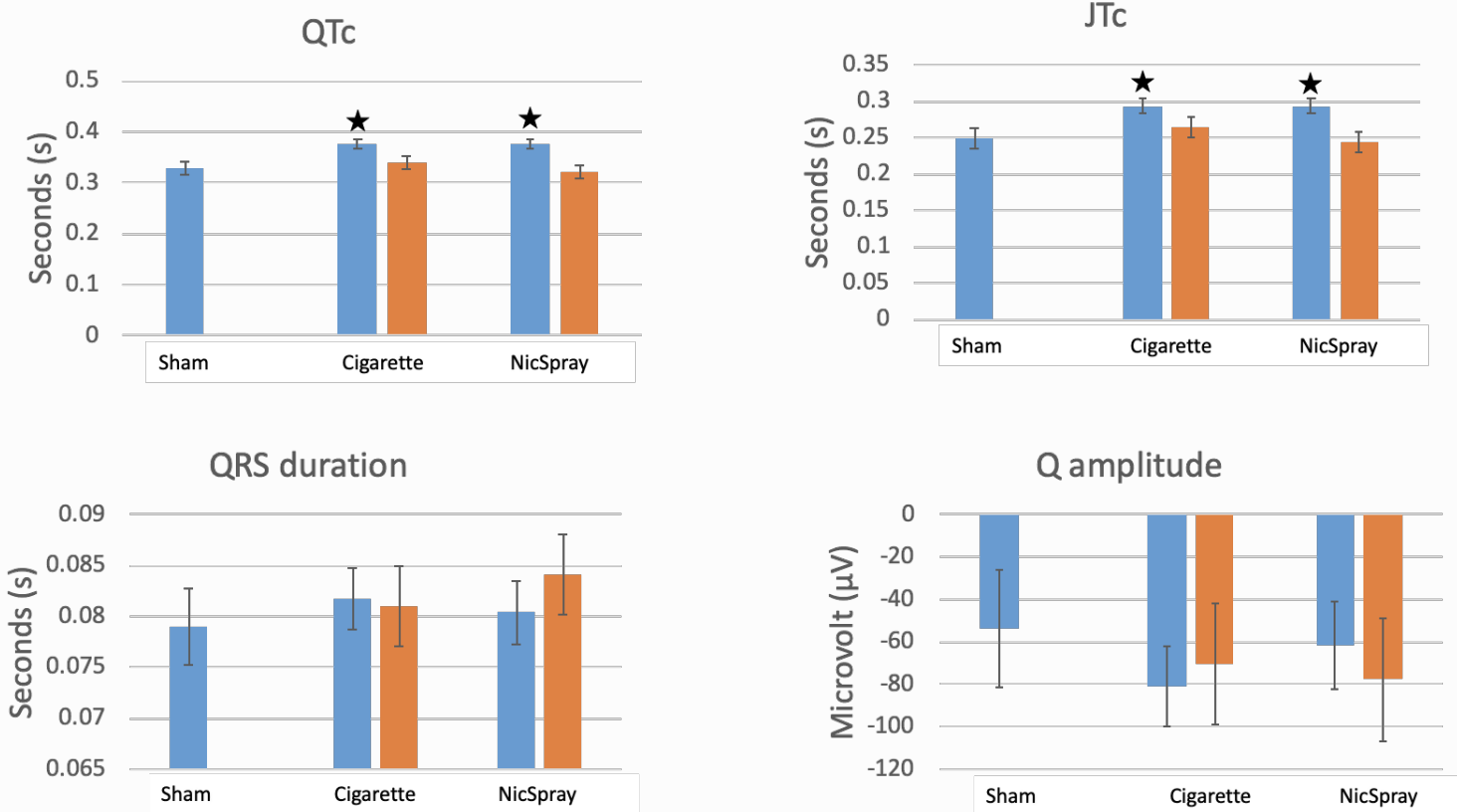

Supplementary Figure 3.3 The Area Under the Curve for the baseline ECG parameters

for each visit day among smokers 
$\rightarrow$ Sham

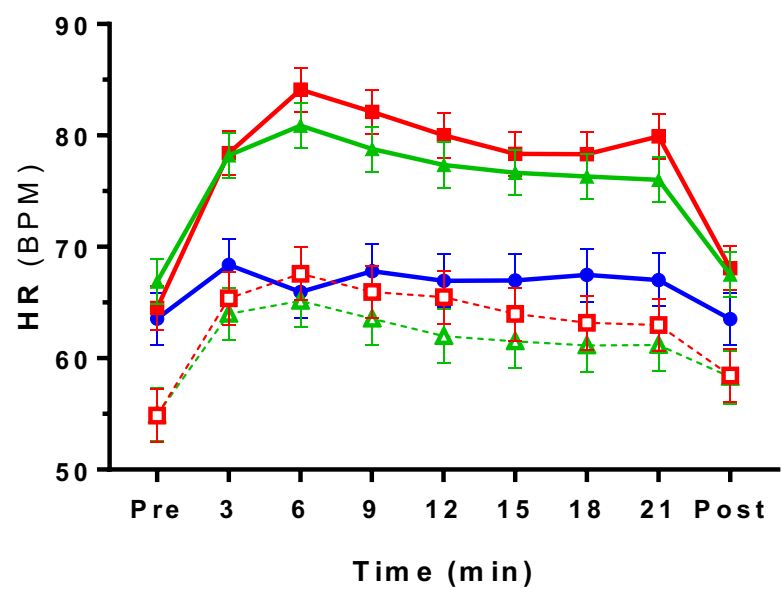

\&

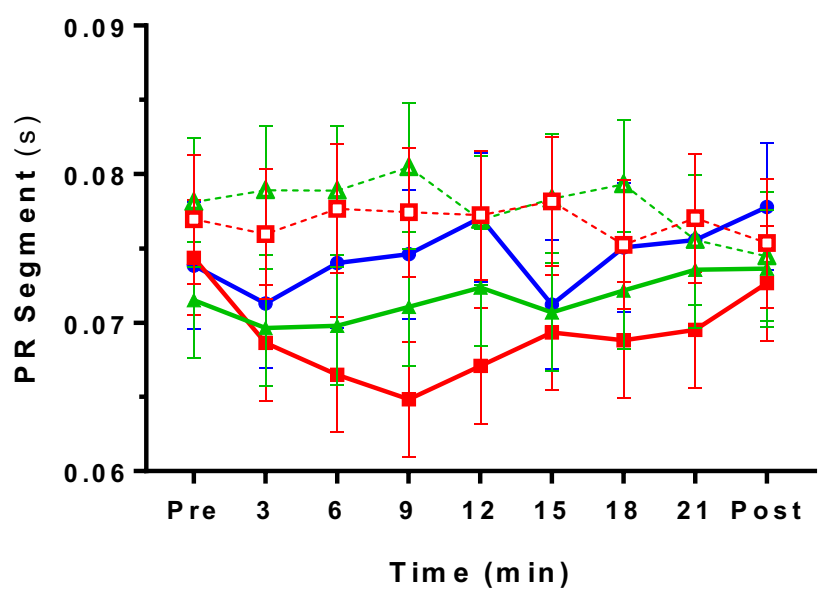

$\rightarrow$ Cigarette
- B B-Cig
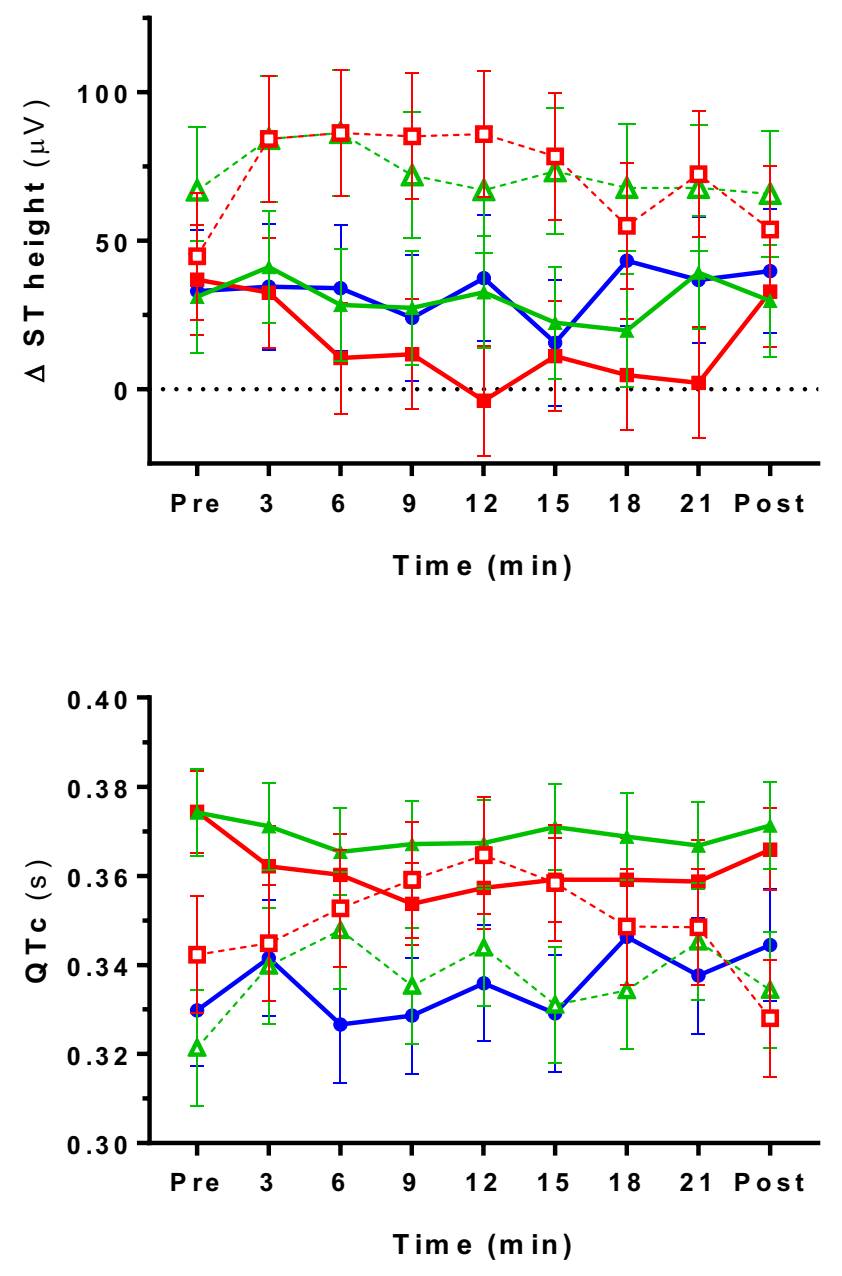

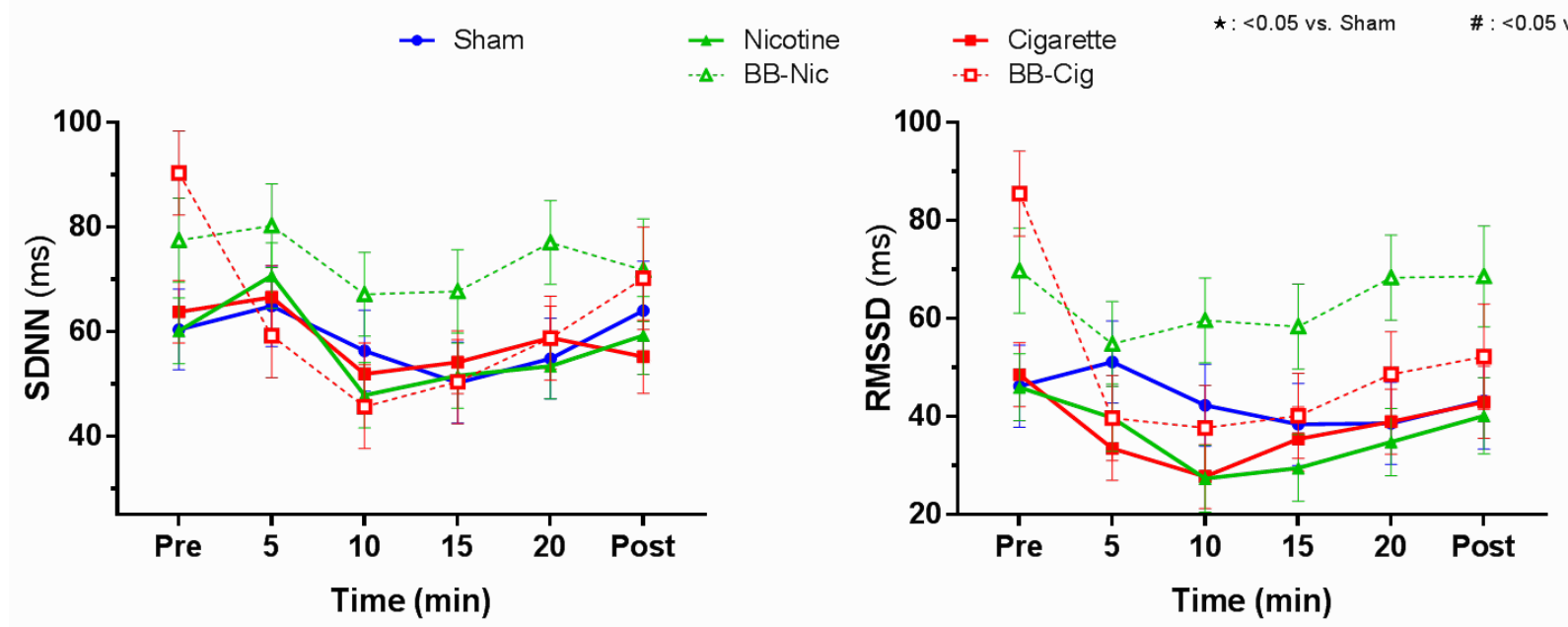

$\infty$
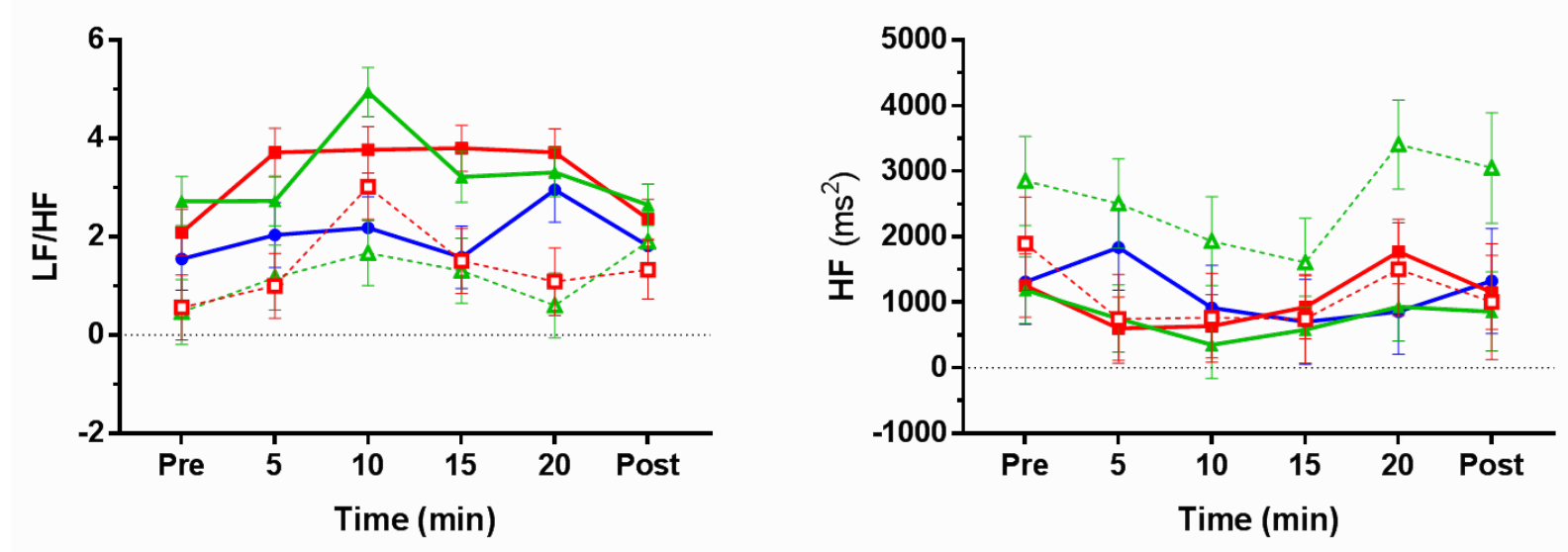

Supplementary Figure 3.4 The raw values of several ECG parameters in pre-post and during exposure days among smokers 

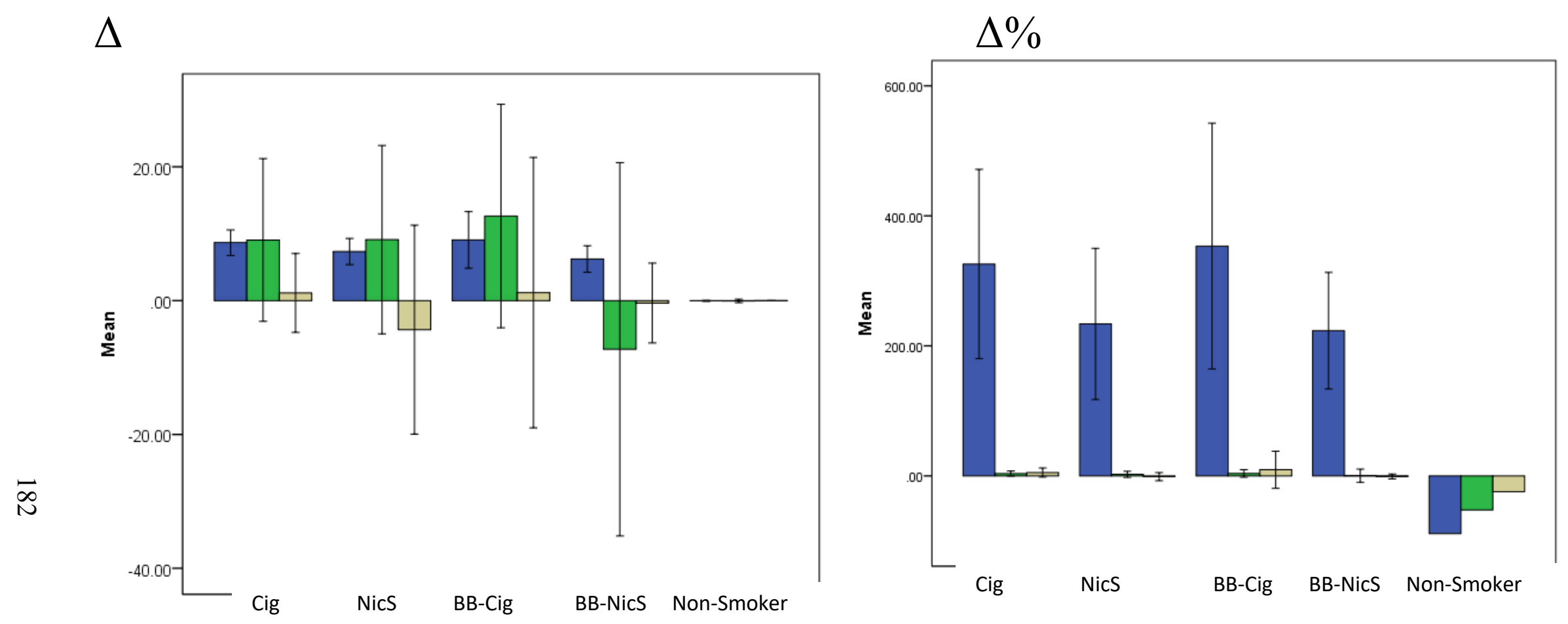

Difference between pre and post exposure:

$\square$ Nicotine

$\square$ Cotinine

$\square$ 3HC (3-hyoxycotinine)

Supplementary Figure 3.5 The raw $(\Delta)$ and relative $(\Delta \%)$ changes with standard errors of nicotine, Cotinine and 3-hyroycotinine (3$\mathrm{OH})$ levels from baseline per study visit 

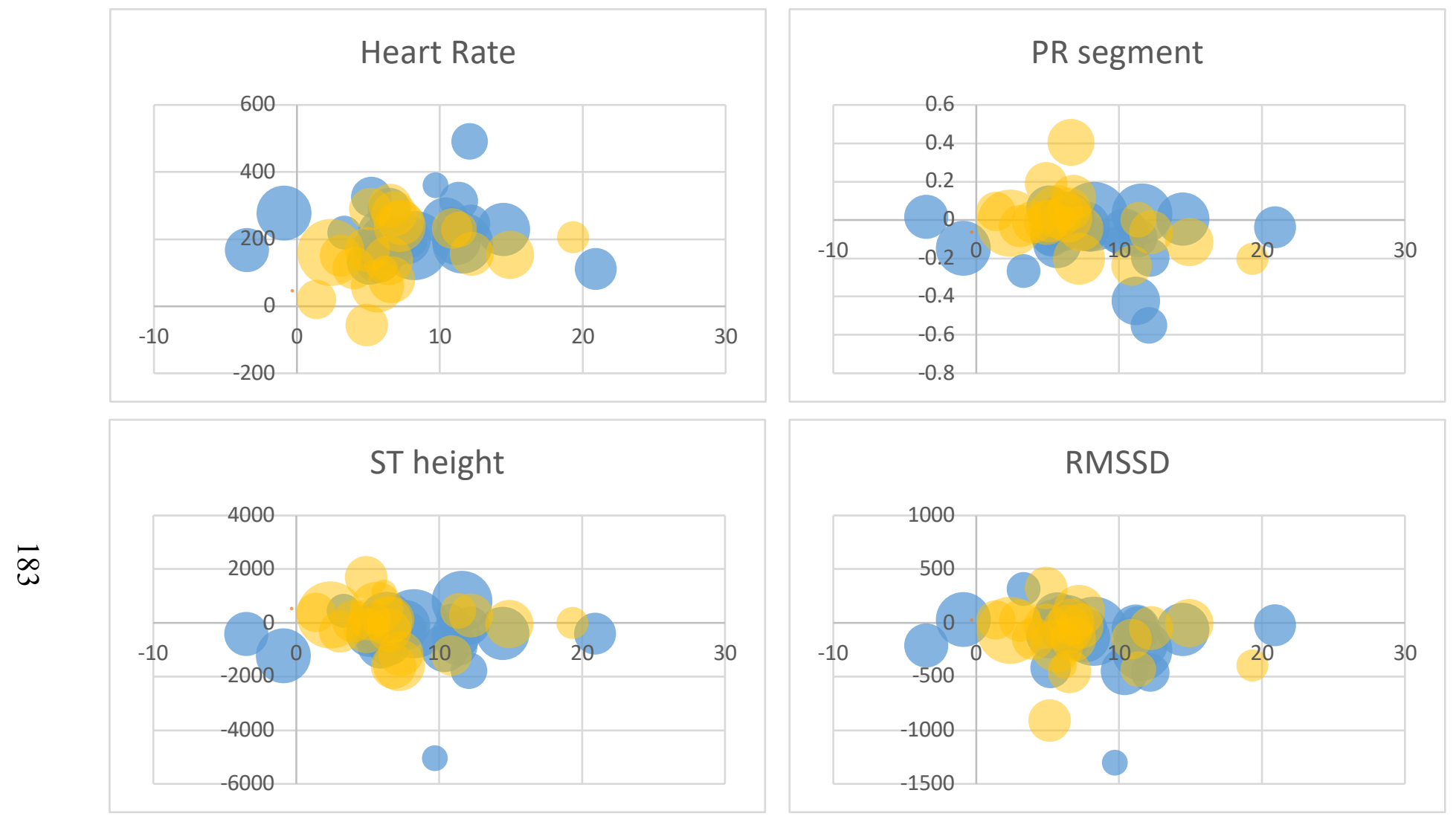

Supplementary Figure 3.6 The relationship between Area Under the Curves (AUC) of ECG parameters (Heart Rate, PR segment, ST height and root mean squared of successive differences (RMSSD)), with changes in Nicotine and Baseline Cotinine levels

$\mathrm{X}$ axis $-\Delta$ Nicotine $(\mathrm{ng} / \mathrm{ml})$

$\mathrm{Y}$ axis - AUC of $\Delta \mathrm{ECG}$ parameter

$\mathrm{Z}$ axis - Relative size of bubble indicates baseline cotinine level

\section{Cigarette}

NicSpray

Non-smokers 
(A)

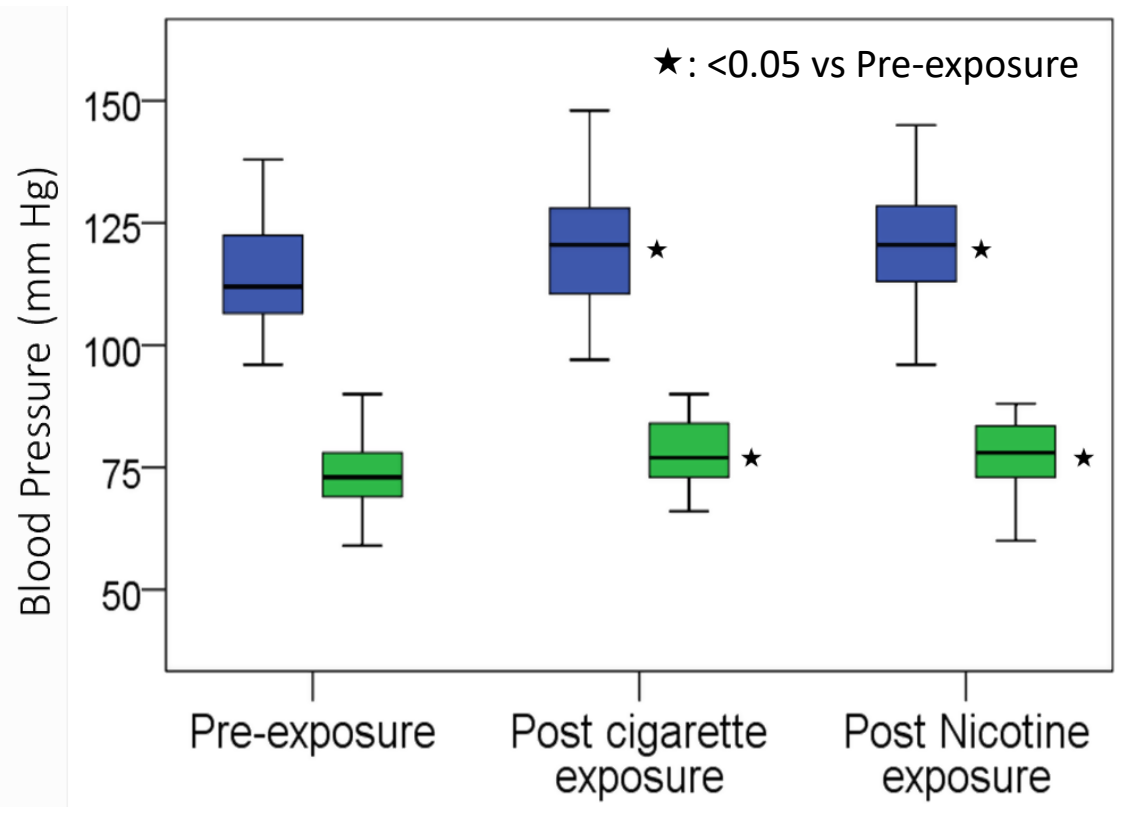

Supplementary Figure 3.7 The changes in Blood Pressure post cigarette and Nicotine Spray use (A) and their correlation with change in nicotine levels (B)

There is no significant difference in pre-exposure and post exposure Blood pressure between Cig and NicS days $(\mathrm{p}>0.05)$.

(B)

\begin{tabular}{lcc}
\hline & $\begin{array}{c}\text { 20 smokers (Cig-day } \\
\text { and NicS-day) and } \\
\text { 9 non-smokers }\end{array}$ & $\begin{array}{c}\beta \text {-Blocker Cigarette and } \\
\beta \text {-Blocker Nicotine days } \\
\mathrm{N}=20\end{array}$ \\
\hline Systolic Blood Pressure & 0.438 & 0.180 \\
Diastolic Blood Pressure & $\mathrm{P}=0.001$ & $\mathrm{P}=0.447$ \\
& 0.374 & -0.160 \\
& $\mathrm{P}=0.008$ & $\mathrm{P}=0.500$ \\
\hline
\end{tabular}




\title{
Appendix B
}

\author{
Visit: Day $1 \square$ or Day 2
}

Subject ID:

DOB:

Outside Temp:

Humidity:

Date: $\ldots 1 \ldots 1 \ldots$ Time of arrival:

Consent / abstained from food / beverages (Except water) for 8 hours and from nicotine/Cig for 8 hours? Y/N

Date and time for last Cigarette/E-cig or other nicotine product use: Date:

Time: Height Weight

Record START time for each event

\begin{tabular}{|c|c|c|c|c|c|c|}
\hline $\begin{array}{l}\text { Rest } 5 \text { mins } \\
\text { (consent) } \\
\text { BP and HR } \\
\text { (sitting) }\end{array}$ & $\begin{array}{l}\text { Rest } 5 \text { mins } \\
\text { (reclining) } \\
\text { ECG } \\
12 \mathrm{sec} \text { and } \\
5 \text { mins }\end{array}$ & IV blood draw & $\begin{array}{l}\text { Walk outside } \\
\text { and rest } 5 \\
\text { mins } \\
\text { (sitting) } \\
\text { Start ECG } \\
\text { after rest }\end{array}$ & $\begin{array}{l}\text { Smoke/ } \\
\text { Nicotine/ Straw } \\
\text { outside } \\
\text { (sitting) }\end{array}$ & $\begin{array}{l}\text { Record } 20 \\
\text { mins ECG } \\
\text { outside } \\
\text { including the } \\
\text { exposure time } \\
\text { (sitting) }\end{array}$ & $\begin{array}{l}\text { Walk } \\
\text { inside }\end{array}$ \\
\hline $\begin{array}{l}1 . \\
\text { Time:__ } \\
\text { SBP } \\
\text { DBP } \\
\text { HR }\end{array}$ & $\begin{array}{l}2 . \\
\text { Time: _ : }\end{array}$ & 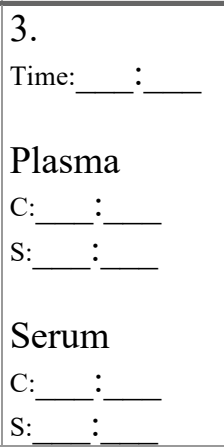 & $\begin{array}{l}4 . \\
\text { Time: }\end{array}$ & $\begin{array}{l}5 . \\
\text { Start } \\
\text { Time:__ }= \\
\text { Stop } \\
\text { Time:__: } \\
\square \text { Cigarette } \\
\square \text { Nicotine } \\
\square \text { Straw }\end{array}$ & $\begin{array}{l}6 . \\
\text { Time: }\end{array}$ & $\begin{array}{l}7 . \\
\text { Time: } \\
- \\
\end{array}$ \\
\hline $\begin{array}{l}\text { Rest } 5 \text { mins } \\
\text { (reclining) } \\
\text { ECG } \\
12 \mathrm{sec} \text { and } \\
5 \text { mins }\end{array}$ & $\begin{array}{l}\begin{array}{l}\text { Rest } 5 \text { mins } \\
\text { (questionnaire) }\end{array} \\
\begin{array}{l}\text { BP and HR } \\
\text { (sitting) }\end{array}\end{array}$ & IV blood draw & $\begin{array}{l}\text { Time of } \\
\text { departure }\end{array}$ & & & \\
\hline $\begin{array}{l}2 . \\
\text { Time: }\end{array}$ & $\begin{array}{l}2 . \\
\text { Time:__ } \\
\text { SBP } \\
\text { DBP } \\
\text { HR }\end{array}$ & 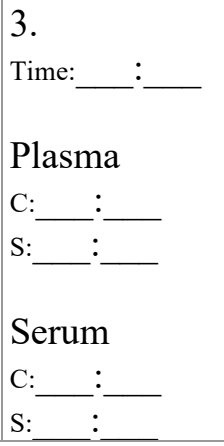 & $\begin{array}{l}7 . \\
\text { Time: }\end{array}$ & & & \\
\hline
\end{tabular}

Recorded by:

Date:

PI Signature:

Date: 
Visit: Day $3 \square$ or Day 4

Subject ID:

DOB:

Outside Temp:

Humidity:

Date: _ _ _ _ _ _ Time of arrival:

Consent / abstained from food / beverages (Except water) for 8 hours and from nicotine/Cig for 8 hours? Y/N

Date and time for last Cigarette/E-cig or other nicotine product use: Date:

Time: Height

$100 \mathrm{mg}$ propranolol Weight

BP and HR every 15 mins for 2 hours

\begin{tabular}{|c|c|c|c|c|c|c|}
\hline $\begin{array}{l}\begin{array}{l}\text { Rest } 5 \text { mins } \\
\text { (consent) }\end{array} \\
\begin{array}{l}\text { BP and HR } \\
\text { (sitting) }\end{array}\end{array}$ & $\begin{array}{l}\text { Rest } 5 \text { mins } \\
\text { (reclining) } \\
\text { ECG } \\
12 \mathrm{sec} \text { and } \\
5 \mathrm{mins}\end{array}$ & IV blood draw & $\begin{array}{l}\text { Walk outside } \\
\text { and rest } 5 \\
\text { mins } \\
\text { (sitting) } \\
\text { Start ECG } \\
\text { after rest }\end{array}$ & $\begin{array}{l}\text { Smoke/ } \\
\text { Nicotine/ Straw } \\
\text { outside } \\
\text { (sitting) }\end{array}$ & $\begin{array}{l}\text { Record } 20 \\
\text { mins ECG } \\
\text { outside } \\
\text { including the } \\
\text { exposure time } \\
\text { (sitting) }\end{array}$ & $\begin{array}{l}\text { Walk } \\
\text { inside }\end{array}$ \\
\hline $\begin{array}{l}1 . \\
\text { Time:__: } \\
\text { SBP } \\
\text { DBP } \\
\text { HR }\end{array}$ & $\begin{array}{l}2 . \\
\text { Time: _ _ : }\end{array}$ & 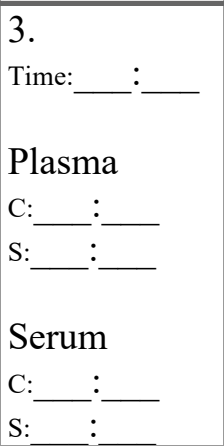 & $\begin{array}{l}4 . \\
\text { Time: }\end{array}$ & $\begin{array}{l}5 . \\
\text { Start } \\
\text { Time:__ : } \\
\text { Stop } \\
\text { Time:__ } \quad \text { _ } \\
\square \text { Cigarette } \\
\square \text { Nicotine } \\
\square \text { Straw }\end{array}$ & $\begin{array}{l}6 . \\
\text { Time: }\end{array}$ & $\begin{array}{l}7 . \\
\text { Time: } \\
- \\
\end{array}$ \\
\hline $\begin{array}{l}\text { Rest } 5 \text { mins } \\
\text { (reclining) } \\
\text { ECG } \\
12 \mathrm{sec} \text { and } \\
5 \text { mins }\end{array}$ & 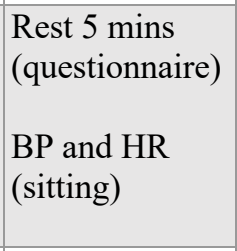 & IV blood draw & $\begin{array}{l}\text { Time of } \\
\text { departure }\end{array}$ & & & \\
\hline $\begin{array}{l}2 . \\
\text { Time: } \quad:\end{array}$ & $\begin{array}{l}2 . \\
\text { Time: }:- \\
\text { SBP } \\
\text { DBP } \\
\text { HR }\end{array}$ & 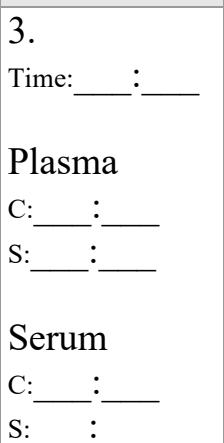 & $\begin{array}{l}7 . \\
\text { Time: } \quad:\end{array}$ & & & \\
\hline
\end{tabular}

Recorded by:

Date:

PI Signature:

Date: 


\section{Appendix C \\ Protocol on how to use LabChart for ECG measurements}

1. Open file

2. Uncheck the "Times in first Column"

3. Set the default sampling rate to $500 \mathrm{~Hz}$

\section{Click OK}

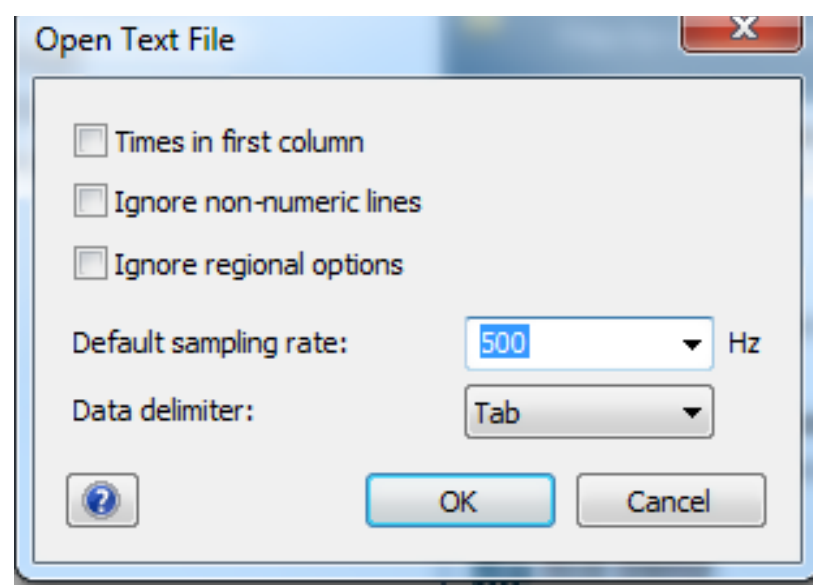

5. Autoscale all channels

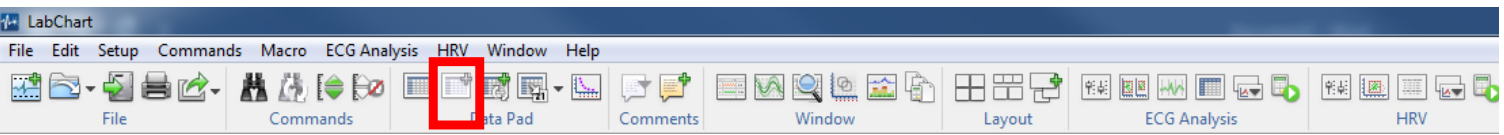

6. Analyze the entire ECG and search and record any arrhythmia, blocks, ectopic beats (PACs, PVCs) Quantify ectopic beats and time relative to exposure. Confirm QRS complex is within $80 \mathrm{~ms}$.

\section{Click ECG settings}

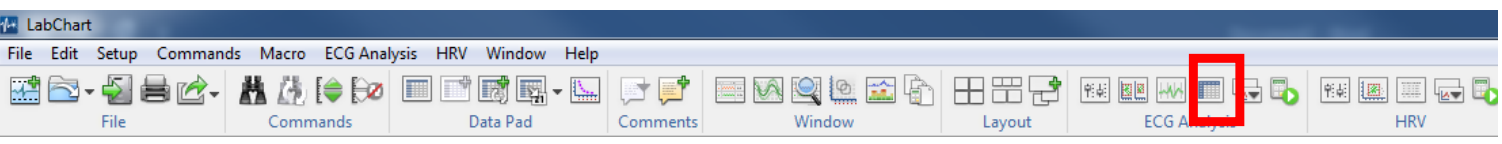




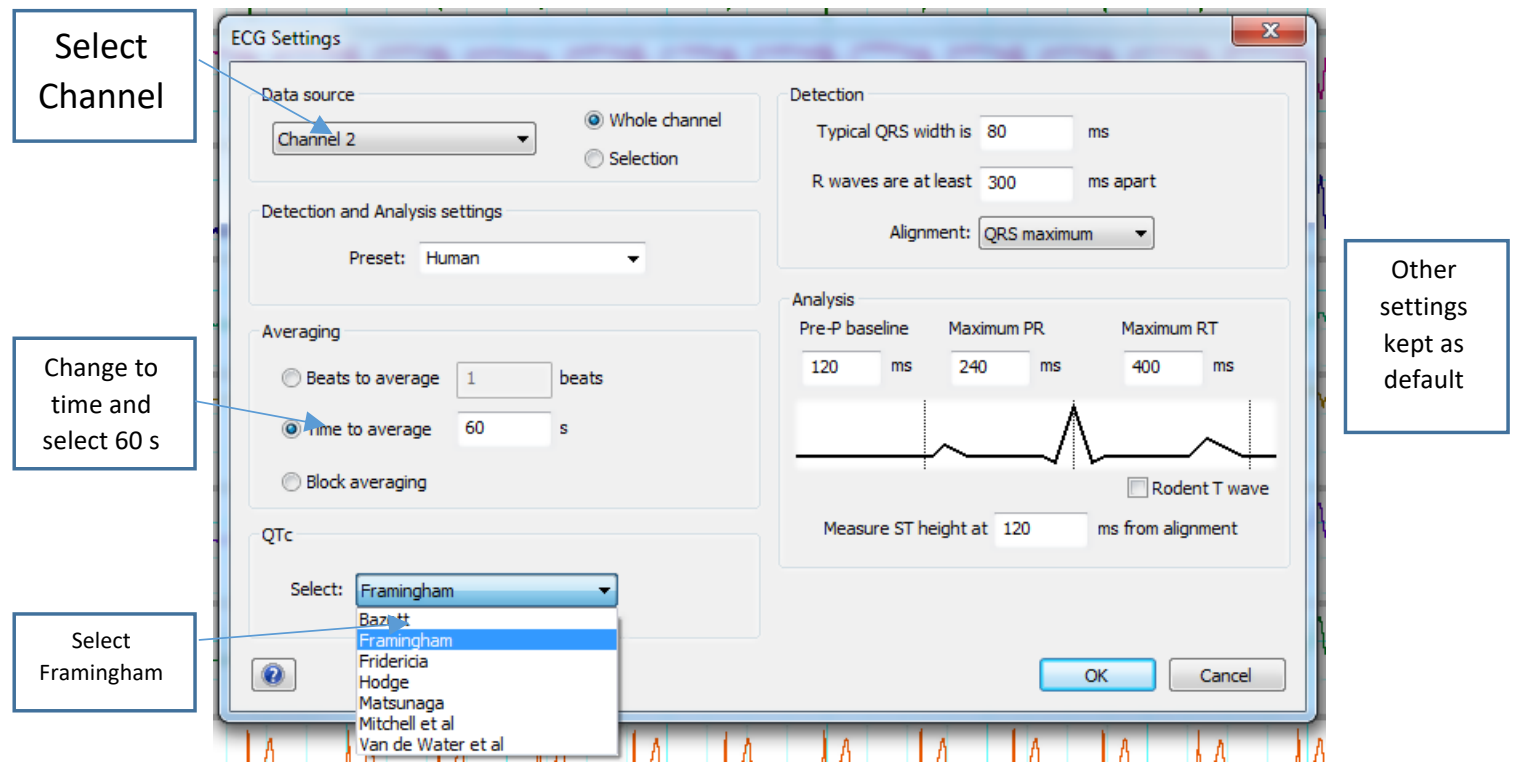

\section{Click ECG Beat Classifier View}
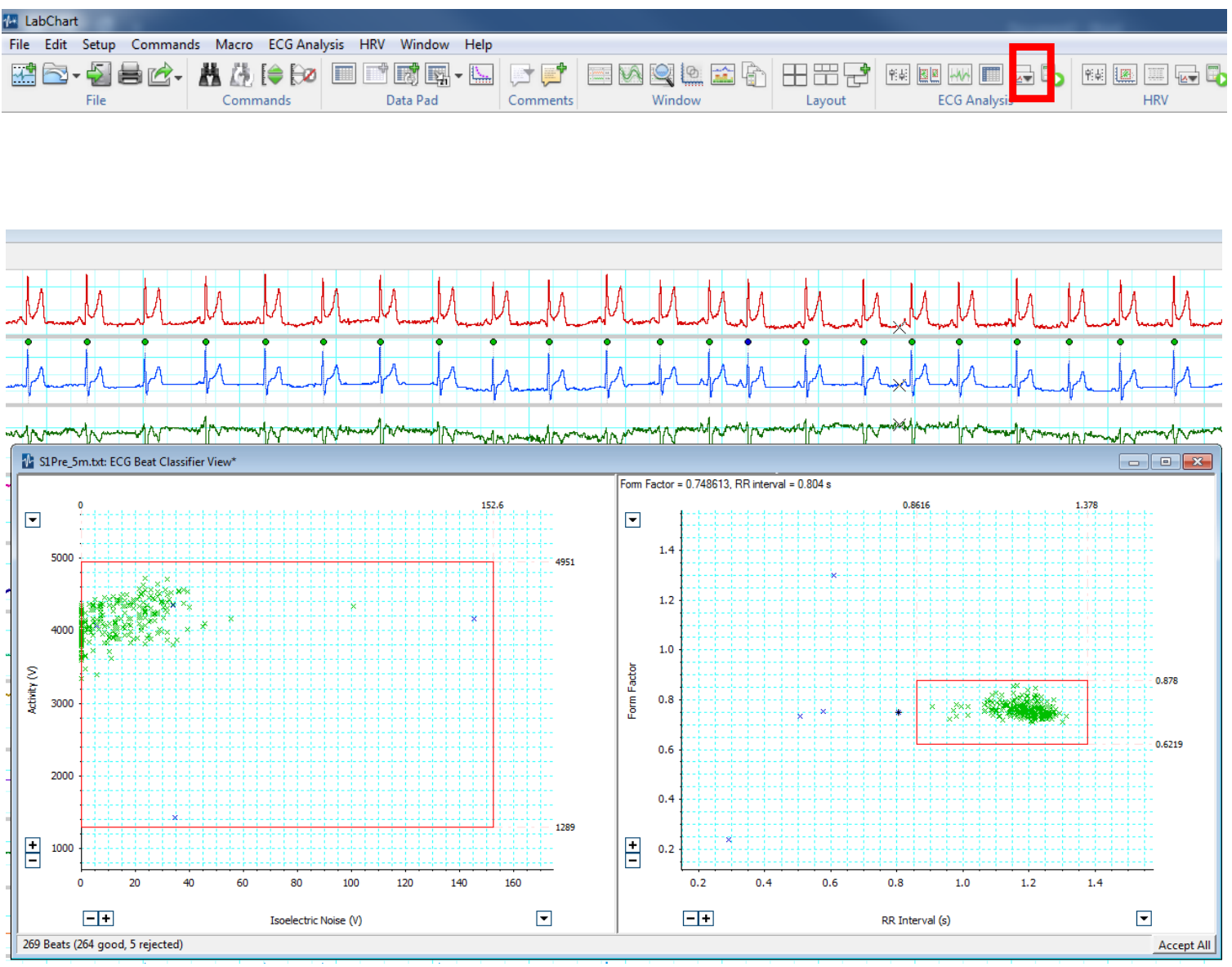
Exclude the outlier beats by mapping the case-specific distribution of activity over the isoelectric noise (in millivolts) and the distribution of the form factor over the RR interval (in seconds).

Click on the marked point and view the beat to decide whether to exclude or not. If excluded then go to the next outlier closer to the data and decide about its inclusion. Keep repeating it until you find a beat which is normal and should be included. Perform this at each four quadrants and using both; Form Factor and Activity.

9. Scroll the entire ECG recording and ensure that all selected beats should be included in the analysis

\section{Click ECG Table View}

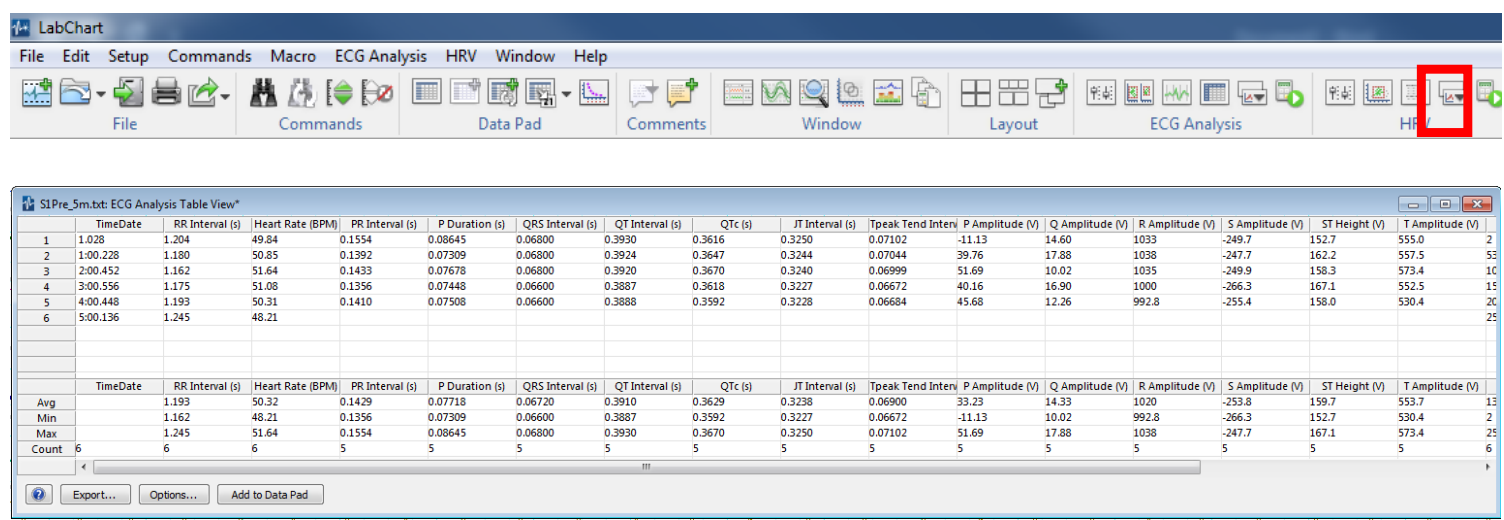




\section{Appendix D}

Nicotine and metabolites determination in human plasma

\subsection{Chemicals and reagents}

(-)-Nicotine (NIC), (-)-Cotinine (COT), and LC-MS Ultra grade ammonium formate were purchased from Sigma-Aldrich (St. Louis, MO). Trans-3'-Hydroxy Cotinine (3HC), and trans-3'-Hydroxy Cotinine- $\mathrm{d}_{3}\left(3 \mathrm{HC}-\mathrm{d}_{3}\right)$ were purchased from Toronto Research Chemicals, (Toronto, Canada). $( \pm)-$ Nicotine- $\mathrm{d}_{3} \quad\left(\mathrm{NIC}-\mathrm{d}_{3}\right)$ and $( \pm)$-Cotinine- $\mathrm{d}_{3} \quad\left(\right.$ COT- $\left.\mathrm{d}_{3}\right)$, were purchased from CDN Isotopes (Pointe-Claire, Quebec, Canada). UHPLC-MS grade water, UHPLC-MS grade acetonitrile, and LC-MS grade formic acid were purchased from Thermo Fisher Scientific Inc. (Waltham MA).

\subsection{UPLC-MS/MS analysis}

$\mathrm{NIC}, \mathrm{COT}$, and $3 \mathrm{HC}$ in human plasma samples were measured by UPLC-MS/MS using a Xevo TQ-S micro quadrupole mass spectrometer with an ESI ionization source, interfaced with Waters Acquity Class-H UPLC equipped with a quaternary pump system (Waters, MA).

After the sample was thawed on ice, $20 \mu \mathrm{l}$ of plasma was mixed with $480 \mu \mathrm{l}$ of $75 \%$ acetonitrile with isotopic labeled internal standard $\left(C O T-d_{3}\right.$, NIC- $d_{3}$, and $\left.3 H C-d_{3}\right)$ in a 1.7 $\mathrm{ml}$ microcentrifuge tube. After incubation on ice for $30 \mathrm{~min}$, the mixture was centrifuged at $4^{\circ} \mathrm{C}$ for $30 \mathrm{~min}$, and $2 \mu \mathrm{l}$ of the supernatant was applied on the UPLC-MS/MS instrument. The separation was performed on an Acquity UPLC BEH HILIC $(50 \mathrm{~mm} \times 2.1 \mathrm{~mm}, 1.7$ $\mu \mathrm{m}$ ) column (Waters, MA) with a binary gradient comprised of $95 \%$ water $5 \%$ ACN $10 \mathrm{mM}$ ammonium formate $\mathrm{pH} 3$ (Solvent A) and 0.1\% formic acid in acetonitrile (Solvent B) at a flow rate $0.4 \mathrm{ml} / \mathrm{min}$. The gradient started at $96.5 \%$ for $1.2 \mathrm{~min}$, ramped down to $30 \%$ 
solvent $\mathrm{B}$ over a 3 min period. Then the gradient ramped down to $0 \% \mathrm{~B}$ in $0.1 \mathrm{~min}$, held at 0\% B for $0.3 \mathrm{~min}$, return to $96.5 \% \mathrm{~B}$ in $0.1 \mathrm{~min}$, and held for $5.3 \mathrm{~min}$ until next injection. Chromatography was performed at $40^{\circ} \mathrm{C}$. The total chromatographic run time was $10 \mathrm{~min}$. The mass spectrometry detector worked in positive ion mode. Optimized cone voltage and collision energy were used for each of the individual analytes. For each analyte, three multiple reaction monitoring (MRM) transitions were set up: one for quantification, one for confirmation, and one for labeled internal standard. Transitions for NIC are $163>130$ for quantification, $163>117$ for confirmation, and $166>130$ for internal standard (NIC$\left.d_{3}\right)$. Transitions for COT are $177>80$ for quantification, $177>98$ for confirmation, and $180>80$ for internal standard $\left(\right.$ COT $\left.-\mathrm{d}_{3}\right)$. Transitions for $3 \mathrm{HC}$ are $193>80$ for quantification, $193>134$ for confirmation, and $196>80$ for internal standard $\left(3 \mathrm{HC}-\mathrm{d}_{3}\right)$. These MRMs were scheduled around the retention time of the analytes. No less than 15 data points were collected for each peak.

TargetLynx quantification application manager software (Waters, MA) was used for peak integration, calibration, and quantification. Analytes in plasma samples were quantified using peak area ratio based on a 7 point-standard curves which were run before and after the plasma samples. 


\section{CURRICULUM VITAE}

Affan B. Irfan

\section{WORK EXPERIENCE}

$7 / 2017-$

Non-invasive Cardiologist

Director, Echocardiography Laboratory, Cabell Huntington Hospital

Site Director for Cardiology Fellowship, Cabell Huntington Hospital

Assistant Professor

Department Cardiology and Clinical Translational Services

Marshall University, Huntington, WV, USA

$2010-2011 \quad$ Clinical Research Fellow

University Hospital Basel, Switzerland

Research Supervisor: Professor Dr. Christian Mueller, MD, FESC Associate

Director, Internal Medicine, University Hospital Basel.

$2009-2010 \quad$ Research Officer

Indus Hospital Research Centre, Pakistan

Research Supervisor: Dr Aamir Javed Khan, M.D., PhD. International Health Johns

Hopkins Bloomberg School of Public Health and Director, Center for Community

Development, Karachi, Pakistan

$2008 \quad$ Research Officer

Iberoamerican Cochrane Center, Hospital de Saint Pau, Spain Research Supervisor: Dr

Pablo Alonso-Coello, M.D.PhD,

Universidad Autónoma de Barcelona, Spain

\section{EDUCATION}

2014-2017

General Cardiology Fellow

Level III Trained Echocardiography

University of Louisville. KY, USA

2011-2014

Internal Medicine Residency Program

University of Illinois - Urbana Champaign, USA 
2003-2008

Bachelor of Medicine and Bachelor of Surgery (M.B.,B.S) Aga Khan

University Medical College, Karachi, Pakistan

2001-2003

General Certificate Examination Advanced Levels

St. Mary's Catholic High School, Dubai, U.A.E.

2001

General Certificate Examination Ordinary Levels

English Medium School Dubai, U.A.E.

\section{CERTIFICATION}

Board Certified American Board of Cardiovascular Diseases

2018

Board Certified American Board of Echocardiography

2018

Board Certified American Society of Nuclear Cardiology

2014

Board Certified American Internal Medicine

2011

US ECFMG Certificate 


\section{PEER REVIEWED PUBLICATIONS (Total 34 with 14 as first or last authors)}

Sympathetic Activation and Nicotine Mediate the Acute Proarrhythmic and Ischemic Effects of Smoking in Humans.

Affan Irfan, George Koromia, Waiel Abusnina, Madison Crank, Lonnie Lucas, Meagen Carter, Nalini Santanam, Andrew DeFilippis, Mark Studeny, Zhengzhi, Pawel

Lorkiewicz, Aruni Bhatnagar, Alex P. Carll

Upcoming

Association between serum cotinine levels and PR and QT interval and their components Affan Irfan, Daniel Riggs, George A. Koromia, Andrew Paul DeFilippis, Elsayed Z.

Soliman, Aruni Bhatnagar, Alex P. Carll

Upcoming

Effects of chronic smoking on atrioventricular conduction and catecholamine production Affan Irfan, Claudia Arab, Andrew P. DeFilippis, Pawel Lorkiewicz, Zhengzhi Xie, Aruni Bhatnagar, Alex P. Carll

Submitted

Clinical Determinants of Myocardial Injury, Detectable and Serial Troponin Levels among Patients with Hypertensive Crisis

Giancarlo Acosta, Ahmed Amro, Rodrigo Aguilar, Waiel Abusnina, Niharika Bhardwaj, George Koromia, Mark Studeny, Affan Irfan

Submitted

Irfan A, Li Y, Bhatnagar A, Soliman EZ. Association between serum cotinine levels and electrocardiographic left atrial abnormality. Ann Noninvasive Electrocardiol. 2019 Jan;24(1):e12586.

Irfan A. Letter by Irfan Regarding Article, "Assessment of the European Society of Cardiology 0-Hour/1-Hour Algorithm to Rule-Out and Rule-In Acute Myocardial Infarction". Circulation. 2017 Apr 18;135(16):e919-e920.

Alok R. Amraotkar; Patrick J. Trainor; Affan Irfan, Charles W. Hargis; Shesh N. Rai; Aruni Bhatnagar; Andrew P. DeFilippis. Presence of multiple coronary angiographic characteristics for the diagnosis of acute coronary thrombus. Cardiol J. 2017;24(1):25-34

Agarwal, A., B. C. Johnston, R. W. Vernooij, A. Carrasco-Labra, R. BrignardelloPetersen, I. Neumann, E. A. Akl, X. Sun, M. Briel, J. W. Busse, S. Ebrahim, C. E. Granados, A. Iorio, A. Irfan, L. Martinez Garcia, R. A. Mustafa, A. Ramirez-Morera, A. Selva, I. Sola, A. J. Sanabrai, K. A. Tikkinen, P. O. Vandvik, Y. Zhang, O. E. Zazueta, Q. Zhou, H. J. Schunemann, G. H. Guyatt and P. Alonso-Coello. "Authors Seldom Report the Most Patient-Important Outcomes and Absolute Effect Measures in Systematic Review Abstracts." J Clin Epidemiol, (2016). Aug 21. [Epub ahead of print]. 
Alonso-Coello P, Carrasco-Labra A, Brignardello-Petersen R, Neumann I, Akl EA, Vernooij RW, Johnston BC, Sun X, Briel M, Busse JW, Ebrahim S, Granados CE, Iorio A, Irfan A, Martínez García L, Mustafa RA, Ramírez-Morera A, Selva A, Solà I, Sanabria AJ, Tikkinen KA, Vandvik PO, Zazueta OE, Zhang Y, Zhou Q, Schünemann H, Guyatt GH. Systematic reviews experience major limitations in reporting absolute effects. J Clin Epidemiol. 2016 Apr;72:16-26.

Akl, E. A., A. Carrasco-Labra, R. Brignardello-Petersen, I. Neumann, B. C. Johnston, X. Sun, M. Briel, J. W. Busse, S. Ebrahim, C. E. Granados, A. Iorio, A. Irfan, L. Martinez Garcia, R. A. Mustafa, A. Ramirez-Morera, A. Selva, I. Sola, A. J. Sanabria, K. A. Tikkinen, P. O. Vandvik, R. W. Vernooij, O. E. Zazueta, Q. Zhou, G. H. Guyatt and P. Alonso-Coello. "Reporting, Handling and Assessing the Risk of Bias Associated with Missing Participant Data in Systematic Reviews: A Methodological Survey." BMJ Open 5, no. 9 (2015): e009368.

Irfan, A., T. Reichlin, R. Twerenbold, C. Fischer, P. Ballarino, B. Nelles, K. Wildi, C. Zellweger, M. Rubini Gimenez, M. Mueller, P. Haaf, A. Fischer, H. Freidank, S. Osswald and Mueller. "Cardiomyocyte Injury Induced by Hemodynamic Cardiac Stress: Differential Release of Cardiac Biomarkers." Clin Biochem 48, no. 18 (2015): 1225-9.

Cho, J. H., G. Kutti Sridharan, S. H. Kim, R. Kaw, T. Abburi, A. Irfan and A. G. Kocheril. "Right Ventricular Dysfunction as an Echocardiographic Prognostic Factor in Hemodynamically Stable Patients with Acute Pulmonary Embolism: A Meta-Analysis." BMC Cardiovasc Disord 14, (2014): 64.

Khan, M. B., R. Kumar, F. B. Irfan, A. B. Irfan and M. E. Bari. "Civilian Craniocerebral Gunshot Injuries in a Developing Country: Presentation, Injury Characteristics, Prognostic Indicators, and Complications." World Neurosurg 82, no. 1-2 (2014): 14-9.

Alonso-Coello, P., A. Carrasco-Labra, R. Brignardello-Petersen, I. Neumann, E. A. Ak1, X. Sun, B. C. Johnston, M. Briel, J. W. Busse, D. Glujovsky, C. E. Granados, A. Iorio, A. Irfan, L. Martinez Garcia, R. A. Mustafa, A. Ramirez-Morera, I. Sola, K. A. Tikkinen, S. Ebrahim, P. O. Vandvik, Y. Zhang, A. Selva, A. J. Sanabria, O. E. Zazueta, R. W. Vernooij, H. J. Schunemann and G. H. Guyatt. "A Methodological Survey of the Analysis, Reporting and Interpretation of Absolute Risk Reduction in Systematic Reviews (Arrow): A Study Protocol." Syst Rev 2, (2013):

113.

Irfan, A., S. Hublikar, J. H. Cho and J. Hill. "The Value of History and Goals of Care with

Code Status; Even in an Emergency Setting." BMJ Case Rep 2013, (2013).

Haaf, P., R. Twerenbold, T. Reichlin, J. Faoro, M. Reiter, C. Meune, S. Steuer, S. Bassetti, R. Ziller, C. Balmelli, I. Campodarve, C. Zellweger, A. Kilchenmann, A. Irfan, J. 
Papassotiriou, B. Drexler and C. Mueller. "Mid-Regional Pro-Adrenomedullin in the Early Evaluation of Acute Chest Pain Patients." Int J Cardiol 168, no. 2 (2013): 1048-55.

Irfan, A., T. Reichlin, R. Twerenbold, M. Meister, B. Moehring, K. Wildi, S. Bassetti, C. Zellweger, M. R. Gimenez, R. Hoeller, K. Murray, S. M. Sou, M. Mueller, T. Mosimann, M. Reiter, P. Haaf, R. Ziller, H. Freidank, S. Osswald and C. Mueller. "Early Diagnosis of Myocardial Infarction Using Absolute and Relative Changes in Cardiac Troponin Concentrations." Am J Med

126, no. 9 (2013): 781-788 e2.

Irfan, A., R. Twerenbold, M. Reiter, T. Reichlin, C. Stelzig, M. Freese, P. Haaf, W. Hochholzer, S. Steuer, S. Bassetti, C. Zellweger, H. Freidank, F. Peter, I. Campodarve, C. Meune and C. Mueller. "Determinants of High-Sensitivity Troponin T among Patients with a Noncardiac Cause of Chest Pain." Am J Med 125, no. 5 (2012): 491-498 e1.

Breidthardt, T*., A. Irfan*, T. Klima, B. Drexler, C. Balmelli, N. Arenja, T. Socrates, R. Ringger, C. Heinisch, R. Ziller, J. Schifferli, C. Meune and C. Mueller. "Pathophysiology of Lower Extremity Edema in Acute Heart Failure Revisited." Am J Med 125, no. 11 (2012): 1124 e1-1124 e8. (*co-first authors)

Meune, C., T. Reichlin, A. Irfan, N. Schaub, R. Twerenbold, J. Meissner, M. Reiter, A. Luthi, P. Haaf, C. Balmelli, B. Drexler, K. Winkler, W. Hochholzer, S. Osswald and C. Mueller. "How Safe Is the Outpatient Management of Patients with Acute Chest Pain and Mildly Increased Cardiac Troponin Concentrations?" Clin Chem 58, no. 5 (2012): 916-24.

Meune, C., R. Twerenbold, B. Drexler, C. Balmelli, C. Wolf, P. Haaf, T. Reichlin, A. Irfan, M. Reiter, C. Zellweger, J. Meissner, C. Stelzig, M. Freese, I. Capodarve and C. Mueller. "Midregional Pro-a-Type Natriuretic Peptide for Diagnosis and Prognosis in Patients with Suspected Acute Myocardial Infarction." Am J Cardiol 109, no. 8 (2012): 1117-23.

Reichlin, T., A. Irfan, R. Twerenbold, M. Reiter, W. Hochholzer, H. Burkhalter, S. Bassetti, S. Steuer, K. Winkler, F. Peter, J. Meissner, P. Haaf, M. Potocki, B. Drexler, S. Osswald and C. Mueller. "Utility of Absolute and Relative Changes in Cardiac Troponin Concentrations in the Early Diagnosis of Acute Myocardial Infarction." Circulation 124, no. 2 (2011): 136-45.

Meissner, J., A. Irfan, R. Twerenbold, S. Mueller, M. Reiter, P. Haaf, T. Reichlin, N. Schaub, K. Winkler, O. Pfister, C. Heinisch and C. Mueller. "Use of Neutrophil Count in Early Diagnosis and Risk Stratification of Ami." Am J Med 124, no. 6 (2011): 534-42.

Meune, C., C. Balmelli, T. Marxer, J. Meissner, R. Twerenbold, M. Reiter, T. Reichlin, P. Haaf, B. Drexler, A. Irfan, C. Stelzig, M. Freese, K. Winkler and C. Mueller. "HighSensitive Troponin, B-Type Natriuretic Peptide and Coronary Angiogram Findings in 
Patients with Non St- Segment Elevation Acute Coronary Syndrome." Int J Cardiol 153, no. 3 (2011): 335-7.

Meune, C., C. Balmelli, R. Twerenbold, T. Reichlin, M. Reiter, P. Haaf, S. Steuer, S. Bassetti, K. Sakarikos, I. Campodarve, C. Zellweger, A. Irfan, B. Drexler and C. Mueller. "Patients with Acute Coronary Syndrome and Normal High-Sensitivity Troponin." Am J Med 124, no. 12 (2011): 1151-7.

Irfan, A., M. J. Zellweger and C. Mueller. "'Universal Definition" Methodology and

Conclusions Are a Concern." J Am Coll Cardiol 58, no. 3 (2011): 313-4; author reply 314-5.

Irfan, A., P. Haaf, J. Meissner, R. Twerenbold, M. Reiter, T. Reichlin, N. Schaub, A. Zbinden, C. Heinisch, B. Drexler, K. Winkler and C. Mueller. "Systolic Blood Pressure at Emergency Department Presentation and 1-Year Mortality in Acute Chest Pain Patients." Eur J Intern Med 22, no. 5 (2011): 495-500.

Alonso-Coello, P., A. Irfan, I. Sola, I. Gich, M. Delgado-Noguera, D. Rigau, S. Tort, X. Bonfill, J. Burgers and H. Schunemann. "The Quality of Clinical Practice Guidelines over the Last Two Decades: A Systematic Review of Guideline Appraisal Studies." Qual Saf Health Care 19, no. 6 (2010): e58.

Bajaj, R. R., A. Mohammad, T. Hong, A. Irfan, W. Sharieff, A. Bagnall, J. A. Christie, M. J. Kutryk, R. J. Chisholm and A. N. Cheema. "Comparison of Abciximab and Eptifibatide on Angiographic and Clinical Outcomes in Rescue Percutaneous Coronary Intervention for Failed Fibrinolytic Therapy." J Invasive Cardiol 22, no. 8 (2010): 34752.

Gallardo, C. R., D. Rigau, A. Irfan, A. Ferrer, J. A. Cayla, X. Bonfill and P. AlonsoCoello. "Quality of Tuberculosis Guidelines: Urgent Need for Improvement." Int J Tuberc Lung Dis 14, no. 8 (2010): 1045-51.

Aly, Z., K. Abbas, S. F. Kazim, F. Taj, F. Aziz, A. Irfan, R. Sheikh, M. Shakir, S. M. Javed and Z. Fatmi. "Awareness of Stroke Risk Factors, Signs and Treatment in a Pakistani Population." J Pak Med Assoc 59, no. 7 (2009): 495-9

Kazim, S. F., Z. Aly, H. K. Bangash, B. P. Harchandani, A. B. Irfan, S. M. Javed, R. Khalil- ur-Rehman, H. Naqvi and M. M. Khan. "Attitudes toward Suicide Bombing in Pakistan." Crisis 29, no. 2 (2008): 81-5.

Jaffe, R., A. Irfan, T. Hong, R. J. Chisholm and A. N. Cheema. "Intraluminal Filling Defects on Coronary Angiography: More Than Meets the Eye." Clin Cardiol 30, no. 9 (2007): 480-4. 


\section{ABSTRACTS / POSTERS (Total 28 with 17 as first or last authors)}

Affan Irfan, Daniel Riggs, George Koromia, Andrew Defilippis, Elsayed Soliman, Aruni Bhatnagar, Alex Carl. Association between Serum Cotinine Levels and PR and QT Intervals and their components. American College of Cardiology Conference, 2019.

Ahmed Amro, Giancarlo Acosta, Kanaan Mansoor, George Koromia, Rodrigo Aguilar, Mehiar El-Hamdani, Affan Irfan. Elevated Diastolic Blood Pressure predicts short-term risk for Acute Coronary Syndrome in patients without prior Cardiovascular Disease. American College of Cardiology Conference, 2019.

Ahmed Amro, Giancarlo Acosta, George Koromia, Niharika Bhardwaj, Rodrigo Aguilar, Mehiar El-Hamdani, Affan Irfan Albumin Level As A Risk Marker And Predictor Of Peripartum Cardiomyopathy. American College of Cardiology Conference, 2019.

Affan Irfan, George Koromia, Waiel Abusnina, Mark Studney, Aruni Bhatnagar, Alex Carll. Comparison of the Acute Autonomic Effects of Mainstream Cigarette Smoke to Oral Nicotine Spray American Heart Association's EPI|Lifestyle 2019

Affan Irfan, Majd Kanbour, Giancarlo Acosta, Mark Studney. The additional cost of inpatient two-day myocardial perfusion scan in obese patients. ICNC (International Conference on Nuclear Cardiology and Cardiac CT. Lisbon 2019

Giancarlo Acosta, Ahmed Amro, Rodrigo Aguilar, Waiel Abusnina, Niharika Bhardwaj, George Koromia, Mark Studeny, Affan Irfan. Clinical Determinants of Myocardial Injury, Detectable and Serial Troponin Levels among Patients with Hypertensive Crisis. American Heart Association's Vascular Discovery 2019

Affan Irfan, George Koromia, Waiel Abusnina, Mark Studney, Aruni Bhatnagar, Alex Carll Comparison of the Acute Autonomic Effects of Mainstream Cigarette Smoke to Oral Nicotine Spray. AHA EPI/Lifestyle 2018

Daniel W. Riggs, Ray Yeager, Alex P. Carll, Affan Irfan, Natasha DeJarnett, Rachel J. Keith, Daniel J. Conklin, Sanjay Srivastava, Shesh N. Rai, Sumanth D. Prabhu, and Aruni Bhatnagar. Residential Greenspace is Associated with Arterial Stiffness and QT interval. University of Louisville 2016

Affan Irfan, Patrick Trainor, Schultz, Andrew DeFilippis. Acrolein, smoking exposure and acute myocardial infarction. University of Louisville 2016

Alok Amraotkar, Patrick Trainor, Charles Hargis, Affan Irfan, Andrew DeFilippis. Evaluation of multiple coronary angiographic characteristics for the diagnosis of acute coronary thrombus. Vascular Research Day poster session, Louisville, April 2015 
Affan Irfan; Raphael Twerenbold; Karin Wildi; Rubini-Gimenez Maria; Cedric Jaeger; Michael Freese; Tobias Reichlin; Christian Mueller. Association of albumin and high sensitive cardiac troponin $t$ and $i$ levels among chest pain patients presenting to emergency department. J Am Coll Cardiol. 2015;65(10_S)

Affan Irfan, Krishan Kataria, Bisma Irfan , Sarwar Orakzai. A case of cardiac amyloidosis with a negative biopsy from a noncardiac site. Clinical \& Experimental Cardiology. 2014

Affan Irfan; Tobias Reichlin; Rapahel Twerenbold; Karin Wildi; Christian Mueller. Hemodynamic cardiac stress induced myocardial ischemia as detected by the release of cardiac biomarkers: cardiac troponin $\mathrm{t}$ and i, ck-mb and myoglobin. J Am Coll Cardiol. 2013;61(10_S):. doi:10.1016/S0735-1097(13)60193-X

Affan Irfan; Tobias Reichlin; Raphael Twerenbold; Karin Wildi; Christian Mueller. Determinants of early changes in high-sensitive troponin levels among patients with nonacute myocardial infarction cause of chest pain. J Am Coll Cardiol. 2013;61(10_S):. doi:10.1016/S0735-

1097(13)60232-6

Affan Irfan; Tobias Reichlin; Raphael Twerenbold; Karin Wildi; Christian Mueller. The prognostic value of absolute and relative changes in cardiac troponin concentrations among non- acute myocardial infarction patients. J Am Coll Cardiol. 2013;61(10_S):. doi:10.1016/S07351097(13)60236-3

Affan Irfan; Tobias Reichlin; Raphael Twerenbold; Karin Wildi; Christian Mueller. Combination of absolute and relative changes in cardiac troponin concentrations in the early diagnosis of acute myocardial infarction. J Am Coll Cardiol. 2013;61(10_S):. doi:10.1016/S0735-

1097(13)60233-8

A. Irfan TR, R. Twerenbold, K. Wildi, C. Mueller: The effect of Proton Pump Inhibitor on Blood pressure parameters among chest pain patients presenting to the Emergency Department. American College of Physicians (San Francisco Conference) April 2013.

A Irfan KN, J. Cho, C. Davies. Nausea and vomiting; Diagnosed by treatment. ACP Downstate conference, October, 2012.

JH Cho, S Kim, T Abburi, A Irfan, A. Kocheril. Right Ventricular Dysfunction as an Echocardiographic Prognostic Factor in Hemodynamically Stable Patients with Acute Pulmonary Embolism: a Meta-Analysis. ACP Downstate conference, October, 2012.

Johnston B, Alonso P, Neumann I, Carrasco A, Petersen RB, Sun X, Akl E, Briel M, Busse J, Dahm P, Granados CE, Glujovsky D, Iorio A, Irfan A, Martinez L, Mustafa R, 
Ramírez A, Sola I, Sultan S, Tikkinen K, Vandvik P, Zhang M, Guyatt. G. Reporting of absolute estimates of effect of patient important benefits and harms in abstracts of Cochrane and non-Cochrane systematic reviews. 20th Cochrane Colloquium, Auckland, New Zealand October 2012.

Alonso P, Carrasco A, Petersen RB, Neumann I, Elie Akl XS, Johnston B, Briel M, Busse J, Dahm P, Granados CE, Glujovsky D, Iorio A, Irfan A, Martinez L, Mustafa R, Ramírez A, Sola I, Sultan S, Tikkinen K, Vandvik P, Zhang M, Guyatt G. Risk difference estimation and reporting in systematic reviews. 20th Cochrane Colloquium, Auckland, New Zealand October 2012.

Akl E, Alonso P, Neumann I, Petersen RB, Johnston B, Sun X, Carrasco A, Briel M, Busse J, Dahm P, Granados CE, Glujovsky D, Iorio A, Irfan A, Martinez L, Mustafa R, Ramírez A, Sola I, Sultan S, Tikkinen K, Vandvik P, Zhang M, Guyatt. G. Reporting, handling, and judging risk of bias associated with missing participant data in systematic reviews. 20th Cochrane Colloquium, Auckland, New Zealand October 2012.

Twerenbold R, Reiter M, Reichlin R, Balmelli C, Cattelan, Drexler B, Irfan A, Winkler K, Heinisch C, Osswald S, Mueller C. Utility of the ST-segment deviation score in the risk prediction of patients with suspected acute myocardial infarction. Congress of the European Society of Cardiology, Paris - France 2011.

Irfan A, Reichlin T, Twerenbold R, Reiter M, Balmelli C, Meissner J, Haaf P, Drexler B, Osswald S, Mueller C. Rule out acute myocardial infarction algorithm among patients with increased high sensitive troponin levels. Congress of the European Society of Cardiology, Paris - France 2011.

Irfan A, Reichlin T, Twerenbold R, Reiter M, Balmelli C, Meissner J, Haaf P, Drexler B, Osswald S, Mueller C. The significance of each traditional WHO criterion used to diagnose acute myocardial infarction. Congress of the European Society of Cardiology, Paris - France 2011.

Breidthardt T, Drexler B, Socrates T, Noveanu M, Haaf P, Cattelan CB, Irfan A, Reichlin T, Potocki M, Mueller CH. Plasma NGAL for the prediction of acute kidney injury in acute heart failure. Congress of the European Society of Cardiology, Paris - France 2011.

Balmelli C, Reiter M, Reichlin T, Twerenbold R, Haaf P, Irfan A, Drexler B, Meune C, Osswald S, Mueller C. Direct comparison of high-sensitive and sensitive cardiac troponin assays for risk stratification in patients with acute chest pain. Congress of the European Society of Cardiology, Paris - France 2011.

Agha SA, Georgiopoulou VV, Kalogeropoulos AP, Giamouzis G, Waheed S, Irfan AB, Rashad MA, Bhatt DV, Laskar S, Smith AL, Butler J. Digoxin and Outcomes in Patients with Advanced Heart Failure on Contemporary Optimal Treatment Circulation 2008;118(S_1027-b). 


\section{BOOK CHAPTER}

Affan Irfan, Wayne 1. Miller, Allan Jaffe. "Using cardiac troponin to assist in the management of patients with congestive heart failure". Heart Failure: The Expert's Approach. Alan S. Maisel, Gerasimos Filippatos. Jaypee Brothers Medical Pub; 1 edition (July 2014)

\section{ONLINE PUBLICATIONS}

Irfan Affan, Hirsch GA. What Medication Would You Recommend for a 66-Year-Old Male Who Underwent PCI After MI? http://www.acc.org. April 14, 2015. Accessed Apr 14, 2015. http://www.acc.org/education-and-meetings/patient-case-quizzes/whatmedication-would- you-recommend-for-a-66yo-male-who-underwent-pci-after-mi

Irfan Affan, Hirsch GA. Beta-Blocker Therapy in Patients With Stable Coronary Artery Disease. http://www.acc.org. April 14, 2015. Accessed Apr 14, 2015. http://www.acc.org/latest- in-cardiology/articles/2015/04/13/10/13/beta-blockertherapy-in-patients-with-stable-coronary- artery-disease

\section{HONORS / AWARDS}

GRANT

Marshall University Clinical Translational Services

Role: Principal Investigator

Project: Acute effects of Smoking and Nicotine on electrocardiogram

Award: \$50,000

State: Funded for 2017-2019 
West Virginia Clinical \& Translational Science Institute

Role: Co-Principal Investigator

Project: Development of a statewide integrated model for analysis of clinical \& economic patterns of injection drug use-associated infective endocarditis

Award: \$49,938

State: Funded for 2019-2020 